\title{
O fluxo espectral de caminhos de operadores de Fredholm auto- adjuntos em espaços de Hilbert
}

\author{
Jeovanny de Jesus Muentes Acevedo
}

\author{
DisSERTAÇÃO APRESENTADA \\ AO
Instituto DE MATEMÁtica E Estatística \\ DA \\ Universidade De SÃo Paulo \\ PARA
OBTENÇÁO DO TÍTULO \\ $\mathrm{DE}$ \\ Mestre EM Cî̂NCIAS
}

Área de Concentração: Matemática
Orientador: Prof. Dr. Pierluigi Benevieri

Durante o desenvolvimento deste trabalho o autor recebeu auxílio financeiro da $\mathrm{CNPq}$

São Paulo, outubro de 2013 


\section{O fluxo espectral de caminhos de operadores de Fredholm auto- adjuntos em espaços de Hilbert}

Esta dissertação trata-se da versão original

do aluno Jeovanny de Jesus Muentes Acevedo. 


\section{Resumo}

O objetivo principal desta dissertação é apresentar o fluxo espectral de um caminho de operadores de Fredholm auto-adjuntos em um espaço de Hilbert e suas propriedades.

Pelos resultados clássicos de teoria espectral, sabemos que se $H$ é um espaço de Hilbert e $L: H \rightarrow H$ é um operador linear, limitado e auto-adjunto, $H$ pode ser escrito como soma direta ortogonal

$$
H=H_{+}(L) \oplus H_{-}(L) \oplus \operatorname{Ker} L,
$$

onde $H_{+}(L)$ e $H_{-}(L)$ são os subespaços espectrais positivo e negativo de $L$, respectivamente.

No trabalho damos uma definição de fluxo espectral baseada na decomposição acima, aprofundando as conexões deste conceito com a teoria espectral dos operadores de Fredholm em espaços de Hilbert.

Entre as propriedades do fluxo espectral, será analisada a invariância homotópica que se apresenta em várias formas. Veremos o conceito de índice de Morse relativo, que estende o clássico índice de Morse, e sua relação com o fluxo espectral.

A construção do fluxo espectral dada neste trabalho segue a abordagem de P. M. Fitzpatrick, J. Pejsachowicz e L. Recht em [9].

Palavras-chave: fluxo espectral, índice de Morse, operadores de Fredholm, espaços de Hilbert, teoria espectral. 


\section{Abstract}

The main purpose of this dissertation is to present the spectral flow of a path of selfadjoint Fredholm operators in a Hilbert space and its properties. By classical results in spectral theory, we know that, if $H$ is a Hilbert space and $L: H \rightarrow H$ is a bounded self-adjoint linear operator, $H$ may be written as the following orthogonal direct sum

$$
H=H_{+}(L) \oplus H_{-}(L) \oplus \operatorname{Ker} L,
$$

where $H_{+}(L)$ and $H_{-}(L)$ are the positive and negative spectral subspaces of $L$, respectively.

In this work we give a definition of spectral flow which is based on the above splitting, examining in depth the connection between this concept and the spectral theory of Fredholm operators in Hilbert spaces.

Among the properties of the spectral flow we will analyze the homotopic invariance, which appears on different ways. We will see the concept of relative Morse index, which generalize the classical Morse index, and its relation with the spectral flow.

The construction of the spectral flow given in this work follows the approach of $\mathrm{P}$. M. Fitzpatrick, J. Pejsachowicz and L. Recht in [9].

Key-words: spectral flow, Morse index, Fredholm operators, Hilbert spaces, spectral theory. 


\section{Conteúdo}

Lista de Símbolos $\quad$ i

Introdução $\quad$ iv

1 Preliminares $\quad 1$

1.1 Alguns resultados clássicos da análise funcional . . . . . . . . . . . . . 1

1.2 Soma, produto e quociente de espaços vetoriais e normados . . . . . . . 7

1.3 Matriz de operadores . . . . . . . . . . . . . . . . . . . . 13

1.4 Espaços métricos compactos . . . . . . . . . . . . . . 16

2 Operadores de Fredholm e compactos $\quad 20$

2.1 Operadores de Fredholm em espaços vetoriais reais . . . . . . . . . . . 21

2.2 Operadores de Fredholm em espaços de Banach . . . . . . . . . . . . . 29

2.3 Operadores compactos em espaços de Banach . . . . . . . . . . . . . 33

2.4 Operadores congruentes módulo operador compacto . . . . . . . . . . . 40

3 Operadores de Fredholm em espaços de Hilbert $\quad 44$

3.1 Preliminares: algumas propriedades dos espaços de Hilbert . . . . . . . 45

3.2 Operadores em espaços de Hilbert . . . . . . . . . . . . . . . . . . . . . 48

3.3 Noções básicas da teoria espectral em espaços normados . . . . . . . . 55

3.4 Operadores de Fredholm auto-adjuntos em espaços de Hilbert . . . . . 72

4 Assinatura e índice de Morse relativo $\quad \mathbf{8 6}$

4.1 A assinatura em espaços de Hilbert de dimensão finita . . . . . . . . . 87

4.2 A assinatura generalizada para perturbações compactas auto-adjuntas de uma simetria . . . . . . . . . . . . 93

4.3 Funções de operadores . . . . . . . . . . . . . . . . . . . . . . . . . 104

4.4 Pares de operadores de Fredholm e índice de Morse relativo . . . . . . . 122

$\begin{array}{lll}5 & \text { O fluxo espectral } & 137\end{array}$

5.1 Parametrix cogradiente . . . . . . . . . . . . . . . . 138

5.2 Fluxo espectral de caminhos de operadores de Fredholm auto-adjuntos 151 
5.3 Fluxo espectral para caminhos gerais de operadores de Fredholm autoadjuntos . . . . . . . . . . . . . . . . 160

5.4 Fluxo espectral em pontos singulares isolados . . . . . . . . . . . . 166

Bibliografia 


\section{Lista de Símbolos}

\begin{tabular}{|c|c|c|}
\hline$A^{\perp}$ & 45 & conjunto ortogonal de $A$ \\
\hline$A \backslash B$ & 8 & $\begin{array}{l}\text { conjunto dos pontos que estão em } A \text { que } \\
\text { não estão em } B\end{array}$ \\
\hline $\mathbb{C}$ & 1 & corpo dos números complexos \\
\hline$c_{0}$ & 36 & $\begin{array}{l}\text { espaço das sequências com valores em } \mathbb{R} \\
\text { que convergem a } 0\end{array}$ \\
\hline$c_{00}$ & 36 & espaço das sequências quase nulas em $c_{0}$ \\
\hline $\operatorname{coKer} L$ & 21 & conúcleo do operador $L$ \\
\hline$d\left(x_{0}, E\right)$ & 7 & distância de $x_{0}$ a $E_{1}$ \\
\hline$E_{1}+E_{2}$ & 7 & soma algébrica dos espaços $E_{1}$ e $E_{2}$ \\
\hline$E_{1} \oplus E_{2}$ & 7 & soma direta dos espaços $E_{1}$ e $E_{2}$ \\
\hline$E_{1} \times E_{2}$ & 9 & produto cartesiano dos espaços $E_{1} \times E_{2}$ \\
\hline$E / E_{1}$ & 10 & quociente de dois espaços vetoriais $E$ e $E_{1}$ \\
\hline$\widehat{E}$ & 57 & complexificação de um espaço real $E$ \\
\hline$f^{\prime}\left(\lambda_{0}\right)$ & 167 & diferencial de $f$ em $\lambda_{0}$ \\
\hline$f^{(k)}$ & 108 & derivada $k$-ésima de $f$ \\
\hline$f^{\star}(\pi)$ & 139 & pullback induzido por $f$ e o fibrado $\pi$ \\
\hline$\dot{f}\left(\lambda_{0}\right)$ & 167 & derivada de um caminho $f$ em $\lambda_{0}$ \\
\hline$F(E, F)$ & 21 & $\begin{array}{l}\text { espaço dos operadores de } E \text { em } F \text { com } \\
\text { imagem de dimensão finita }\end{array}$ \\
\hline$G L(E, F)$ & 4 & $\begin{array}{l}\text { operadores limitados inversíveis de } E \text { em } \\
F\end{array}$ \\
\hline$G L_{S}^{+}(H)$ & 78 & $\begin{array}{l}\text { conjunto dos isomorfismos definidos posi- } \\
\text { tivos }\end{array}$ \\
\hline$\dot{\Gamma}_{\Gamma}^{\circ}$ & 105 & interior de uma curva fechada $\Gamma$ \\
\hline$\left[\Gamma\left(t_{1}\right), \Gamma\left(t_{2}\right)\right]$ & 105 & imagem $\Gamma\left(\left[t_{1}, t_{2}\right]\right)$ \\
\hline $\mathcal{G}$ & 140 & conjunto $G L(H) \times K_{S}(H)$ \\
\hline$H_{+}$ & 93 & $\begin{array}{l}\text { subespaço espectral positivo da simetria } \\
\mathcal{J}\end{array}$ \\
\hline$H_{-}$ & 93 & $\begin{array}{l}\text { subespaço espectral negativo da simetria } \\
\mathcal{J}\end{array}$ \\
\hline$H_{-}(L)$ & 69 & subespaço espectral negativo de $L$ \\
\hline
\end{tabular}




$\begin{array}{ll}H_{+}(L) & 69 \\ \operatorname{ind} L & 21 \\ \operatorname{ind}(P, Q) & 123 \\ \operatorname{Im} L & 1 \\ \int_{\Gamma} f(\zeta) d \zeta & 10 \\ J & 15 \\ \mathcal{J} & 93 \\ K(E, F) & 33\end{array}$

Ker $L$

$K_{S}(H) \quad 93$

$\mathbb{K}$

$\widehat{L} \quad 59$

$L \geq T$

$L_{+} \quad 72$

$L_{-} \quad 72$

$L(E, F) \quad 1$

$L \cong T \quad 40$

$L_{S}(H) \quad 49$

$\left(L_{1}, L_{2}\right) \quad 10$

$\mathcal{L}$

$\ell_{2} \quad 30$

$\Lambda_{\Gamma}$

$\mu(L) \quad 87$

$\mu_{\text {rel }}(L, T) \quad 127$

$\mathbb{N} \quad 8$

$P_{H_{+}(L)} \quad 70$

$P_{H_{-}(L)}$

$\begin{array}{ll}P_{\text {Ker } L} & 70\end{array}$

$\|P\| \quad 106$

$\pi \quad 140$

$\partial \omega$
105

9

1

3

(1)

93

(1)

2

2

2

1 (es)

0

0

30

87

27

70

70

70
106
140

109

subespaço espectral positivo de $L$ índice de um operador de Fredholm $L$ índice de um par de Fredholm $(P, Q)$ imagem do operador $L$ integral da aplicação $f$ na curva $\Gamma$ intervalo $[a, b]$ simetria fortemente indefinida conjunto dos operadores compactos de $E$ em $F$

núcleo do operador $L$

espaço dos operadores compactos autoadjuntos em $H$

corpo dos números reais ou complexos complexificação de um operador real $L$ denota que $L-T$ é um operador não negativo

restição do operador $L$ a seu subespaço espectral positivo

restição do operador $L$ a seu subespaço espectral negativo

espaço dos operadores lineares limitados de $E$ em $F$

congruência módulo operador compacto

espaço dos operadores auto-adjuntos reais produto direto dos operadores $L_{1}$ e $L_{2}$ aplicação $L \mapsto L^{-1}$, onde $L \in G L(E)$ espaço das sequências reais ou complexas $\left(x_{n}\right)_{n=1}^{\infty}$ tais que $\sum_{n=1}^{\infty}\left|x_{n}\right|^{2}$ converge comprimento e uma curva retificável $\Gamma$ dimensão do subespaço espectral negativo de $L$

índice de Morse relativo do par $(L, T)$ conjunto dos números naturais projeção ortogonal sobre o subespaço espectral positivo de $L$

projeção ortogonal sobre o subespaço espectral negativo de $L$

projeção ortogonal sobre o núcleo de $L$ norma da partição $P$

fibrado localmente trivial com fibra $\pi^{-1}(\mathcal{J})$

fronteira de $\omega$ 


\begin{tabular}{|c|c|c|}
\hline$\Phi(E, F)$ & 29 & $\begin{array}{l}\text { conjunto dos operadores de Fredholm de } \\
E \text { em } F\end{array}$ \\
\hline$\Phi_{n}(E, F)$ & 29 & $\begin{array}{l}\text { conjunto dos operadores de Fredholm de } \\
E \text { em } F \text { de índice } n\end{array}$ \\
\hline$\Phi_{S}(H)$ & 44 & $\begin{array}{l}\text { conjunto dos operadores de Fredholm } \\
\text { auto-adjuntos }\end{array}$ \\
\hline$\Phi_{S}^{+}(H)$ & 72 & $\begin{array}{l}\text { conjunto dos operadores de Fredholm } \\
\text { auto-adjuntos essencialmente positivos }\end{array}$ \\
\hline$\Phi_{S}^{-}(H)$ & 73 & $\begin{array}{l}\text { conjunto dos operadores de Fredholm } \\
\text { auto-adjuntos essencialmente negativos }\end{array}$ \\
\hline$\Phi_{S}^{i}(H)$ & 73 & $\begin{array}{l}\text { conjunto dos operadores de Fredholm } \\
\text { auto-adjuntos fortemente indefinidos }\end{array}$ \\
\hline$Q\left(\mathrm{~L}, \lambda_{0}\right)$ & 167 & forma crossing de $\mathrm{L}$ em $\lambda_{0}$ \\
\hline $\mathbb{R}$ & 1 & corpo dos números reais \\
\hline $\mathbb{R}^{+}$ & 72 & conjunto dos números reais positivos \\
\hline $\mathbb{R}^{-}$ & 72 & conjunto dos números reais negativos \\
\hline $\operatorname{Re}(\Omega)$ & 117 & parte real dos elementos do conjunto $\Omega$ \\
\hline$r(L)$ & 108 & raio espectral de $L$ \\
\hline$R(\lambda)$ & 56 & resolvente de $L$, para $\lambda \in \rho(L)$ \\
\hline $\mathcal{R}$ & 142 & aplicação raiz quadrada de operadores \\
\hline$\rho(L)$ & 55 & conjunto resolvente de $L$ \\
\hline$S^{1 / 2}$ & 142 & raiz quadrada não negativa do operador $S$ \\
\hline$S(P, E, f)$ & 106 & $\begin{array}{l}\text { soma de Riemann da partição } P \text {, a escolha } \\
E \text { e a aplicação } f\end{array}$ \\
\hline $\operatorname{sf}(\mathrm{L}, J)$ & 152 & $\begin{array}{l}\text { fluxo espectral do caminho } L \text { no intervalo } \\
J\end{array}$ \\
\hline $\operatorname{sf}\left(L, \lambda_{0}\right)$ & 166 & fluxo espectral de $\mathrm{L}$ através de $\lambda_{0}$ \\
\hline $\operatorname{sign}_{\mathcal{J}}\left(\mathcal{J}+K,\left(e_{i}^{ \pm}\right)_{i=1}^{\infty}\right)$ & 98 & assinatura generalizada de $\mathcal{J}+K$ \\
\hline $\operatorname{sign}_{\mathcal{J}}(\mathcal{J}+K)$ & 99 & assinatura generalizada de $\mathcal{J}+K$ \\
\hline $\operatorname{sign} L$ & 87 & $\begin{array}{l}\text { assinatura de um isomorfismo auto- } \\
\text { adjunto } L\end{array}$ \\
\hline $\operatorname{span}\{A\}$ & 8 & espaço vetorial gerado pelo conjunto $A$ \\
\hline$\sigma(L)$ & 55 & espectro de $L$ \\
\hline$\sigma^{+}(L)$ & 72 & espectro positivo de $L$ \\
\hline$\sigma^{-}(L)$ & 72 & espectro negativo de $L$ \\
\hline$\Sigma(\mathrm{L})$ & 166 & conjunto singular de L \\
\hline$T^{*}$ & 48 & adjunto do operador $T$ \\
\hline$\tau$ & 141 & ação de $\mathcal{G}$ em $\Phi_{S}(H)$ \\
\hline$\Upsilon$ & 79 & ação cogradiente \\
\hline$\varrho$ & 144 & seção do fibrado $\pi$ \\
\hline$\varsigma$ & 141 & ação de $\mathcal{G}$ em si mesmo \\
\hline $\bar{X}$ & 2 & fecho do conjunto $X$ \\
\hline $\mathbb{Z}$ & 29 & conjunto dos números inteiros \\
\hline
\end{tabular}




\section{Introdução}

Um instrumento que se tornou um clássico entre os métodos topológicos em Análise não linear é o conceito de índice de Morse. Suponhamos ter um espaço de Hilbert $H$ e um operador linear auto-adjunto $L: H \rightarrow H$. Sabemos da teoria espectral que $H$ possui a decomposição em soma direta ortogonal

$$
H=H_{+}(L) \oplus H_{-}(L) \oplus \operatorname{Ker} L,
$$

onde $H_{+}(L)$ e $H_{-}(L)$ são os subespaços espectrais positivo e negativo de $L$, respectivamente. Se $\operatorname{dim} H_{-}(L)<\infty$, este número é conhecido como índice de Morse de $L$ e é denotado por $\mu(L)$.

O índice de Morse foi por exemplo aplicado por Mawhin e Willem na abordagem do problema de bifurcação seguinte. Sejam $J=[a, b]$ e $U$ uma vizinhança de $J \times\{0\}$ em $\mathbb{R} \times H$, onde $H$ é um espaço de Hilbert real e separável. Suponhamos que $f: U \rightarrow \mathbb{R}$ seja uma aplicação contínua tal que $f(\lambda, 0)=0$ para todo $\lambda \in J$. Se diz que $\lambda_{0} \in J$ é um ponto de bifurcação para a equação $f(\lambda, x)=0$ se toda vizinhança de $\left(\lambda_{0}, 0\right)$ em U contém ao menos um solução $(\lambda, x)$ da equação tal que $x \neq 0$.

Consideremos agora uma aplicação $\psi: U \rightarrow \mathbb{R}$ de classe $C^{2}$ tal que, para cada $\lambda \in J, 0$ seja um ponto crítico do funcional $\psi_{\lambda}=\psi(\lambda, \cdot)$. Para $\lambda \in J$, denotemos por $L_{\lambda}$ o Hessiano de $\psi_{\lambda}=\psi(\lambda, \cdot)$ e suponhamos que $L_{a}$ e $L_{b}$ sejam não singulares. Mawhin e Willem em [20] mostram o seguinte resultado.

Teorema 1. Se $L_{\lambda}$ é um operador de Fredholm auto-adjunto, com $\operatorname{dim} H_{-}\left(L_{\lambda}\right)<\infty$, para cada $\lambda \in J, e$

$$
\mu\left(L_{a}\right) \neq \mu\left(L_{b}\right)
$$

existe $\lambda_{0} \in J$ tal que $\left(\lambda_{0}, 0\right)$ é um ponto de bifurcação da equação $\nabla \psi(\lambda, x)=0$.

O fluxo espectral de um caminho $L=\left\{L_{\lambda}\right\}_{\lambda \in J}$ de operadores de Fredholm autoadjuntos cujos extremos são inversíveis, que denotaremos por $\mathrm{sf}(\mathrm{L}, J)$, é um conceito que foi introduzido por Atiyah, Patodi e Singer em [5] e se aplica nos casos onde o índice de Morse é infinito.

Em [9], P. M. Fitzpatrick, J. Pejsachowicz e L. Recht provam o seguinte resultado, que generaliza o teorema acima. 
Teorema 2. Seja $\psi: U \rightarrow \mathbb{R}$ como acima. Se $L_{\lambda}$ é de Fredholm, para todo $\lambda \in J$, e o fluxo espectral do caminho $\left\{L_{\lambda}\right\}_{\lambda \in J}$ é não nulo, então toda vizinhança de $J \times\{0\}$ contém pontos da forma $(\lambda, x)$, onde $x \neq 0$ é um ponto crítico de $\psi_{\lambda}$.

Ao lado do fluxo espectral, um outro conceito que generaliza o índice de Morse é o índice de Morse relativo. Seja $(L, T)$ um par de isomorfismos auto-adjuntos cuja diferença é compacta. Neste caso, $\operatorname{dim}\left(H_{-}(L) \cap H_{+}(T)\right)<\infty$ e $\operatorname{dim}\left(H_{-}(T) \cap H_{+}(L)\right)<$ $\infty$, como veremos no Capítulo 4 . O índice de Morse relativo de $(L, T)$ é definido como

$$
\mu_{r e l}(L, T)=\operatorname{dim}\left(H_{-}(L) \cap H_{+}(T)\right)-\operatorname{dim}\left(H_{-}(T) \cap H_{+}(L)\right) .
$$

Na Proposição 5.2.6 mostraremos que, se L é um caminho de operadores de Fredholm auto-adjuntos tal que $L_{\lambda}-L_{a}$ é compacto para todo $\lambda \in J$, então

$$
\operatorname{sf}(\mathrm{L}, J)=\mu_{r e l}\left(\mathrm{~L}_{a}, \mathrm{~L}_{b}\right)
$$

Assim, o seguinte corolário é uma consequência de teorema anterior.

Corolário 0.0.1. Seja $\psi: U \rightarrow \mathbb{R}$ como acima. Assuma que

$$
\nabla \psi(\lambda, x)=A x+C(\lambda, x)
$$

onde $A$ é um operador de Fredholm auto-adjunto e $C$ é uma aplicação compacta. Então, o intervalo $J$ contém pontos de bifurcação para a equação $\nabla \psi(\lambda, x)=0$ sempre que

$$
\mu_{\text {rel }}\left(L_{a}, L_{b}\right) \neq 0 \text {. }
$$

O objetivo desta dissertação é a introdução do fluxo espectral, de sua definição e de algumas de suas propriedades. A abordagem deste trabalho é baseada no artigo de P. M. Fitzpatrick, J. Pejsachowicz e L. Recht: Spectral Flow and Bifurcation of Critical Points of Strongly-Indefinite Functionals, Part I. General Theory, Journal of Functional Analysis, 162, 52-95, Academic Press, (1999).

No primeiro capítulo veremos vários teoremas e resultados conhecidos da análise funcional, da álgebra linear e da topologia geral, que servirão como ferramenta útil em todo o trabalho.

No segundo capítulo trataremos dos operadores de Fredholm em espaços vetoriais (ou de Banach) reais, assim como do índice de um operador de Fredholm. Uma dessas propriedades é que o conjunto dos operadores de Fredholm em espaços de Banach é um subconjunto aberto do espaço dos operadores lineares limitados. Os operadores compactos em espaços de Banach, dos quais lembraremos definição na Seção 3, estão estritamente relacionados com os operadores de Fredholm, como veremos na Seção 4. Tal relação será de grande importância na construção do fluxo espectral. 
O propósito do terceiro capítulo é apresentar algumas das propriedades dos operadores de Fredholm auto-adjuntos em espaços de Hilbert. Para este fim, as duas primeiras seções serão dedicadas a destacar algumas características dos espaços de Hilbert e dos operadores auto-adjuntos. Um dos resultados mais interessantes que veremos neste capítulo diz que o conjunto dos operadores de Fredholm auto-adjuntos possui três componentes conexas, que são: o conjunto dos operadores essencialmente positivos (tais que o subespaço espectral negativo tem dimensão finita), o dos operadores essencialmente negativos (tais que o subespaço espectral positivo tem dimensão finita) e o dos operadores fortemente indefinidos (que tem ambos os subespaços espectrais infinito-dimensionais). Trata-se de um resultado conhecido mas, por outro lado, não fácil de ser encontrado na literatura, razão pela qual decidimos prová-lo.

Na primeira parte do Capítulo 4 veremos a noção de assinatura generalizada para operadores da forma $\mathcal{J}+K$, onde $\mathcal{J}$ um oportuno operador de Fredholm auto-adjunto tal que $\mathcal{J}^{2}=I$ e $K$ é um operador compacto e auto-adjunto. O fluxo espectral será definido usando a assinatura generalizada. Na Seção 4.4 trataremos o índice de Morse relativo e suas propriedades. Apresentaremos na Proposição 4.4 .9 uma relação entre o índice de Morse relativo e o índice de Morse clássico.

O fluxo espectral de caminhos de Fredholm auto-adjuntos será definido no Capítulo 5. Uma das propriedades mais importantes do fluxo espectral, que veremos na Seção 5.2, é a invariância homotópica. No Teorema 5.3.3 provaremos que, se $L=\left\{L_{\lambda}\right\}_{\lambda \in J}$ é um caminho de operadores de Fredholm com $\operatorname{dim} H_{-}\left(\mathrm{L}_{\lambda}\right)<\infty$ para todo $\lambda \in J$, então

$$
\operatorname{sf}(\mathrm{L}, J)=\mu\left(\mathrm{L}_{a}\right)-\mu\left(\mathrm{L}_{b}\right) .
$$

Assim, o Teorema 1, dado acima, se torna uma consequência do Teorema 2.

Finalizaremos o trabalho com a noção de fluxo espectral em pontos singulares isolados de um caminho de operadores de Fredholm auto-adjuntos. 


\section{Capítulo 1}

\section{Preliminares}

Neste capítulo apresentaremos alguns resultados conhecidos que serão utilizados como ferramenta ao longo deste trabalho. A maioria deles não será provada. Damos como pré-requisitos as noções de produto interno, métrica, assim como também os conceitos de espaço vetorial, topológico, métrico, normado e de Banach.

A primeira parte dos preliminares tratará de conceitos clássicos da análise funcional. Teoremas como o da aplicação aberta, o de Hahn-Banach, o de Riesz-Fischer, entre outros, serão recordados.

Na segunda seção lembraremos as definições da soma, produto e o quociente de espaços vetoriais. Usando os teoremas apresentados na primeira seção mostraremos algumas propriedades que possuem o produto e o quociente de espaços de Banach. Além disso, veremos a definição do produto direto de dois operadores lineares.

Na terceira seção veremos que podemos representar um operador linear $L: E \rightarrow F$ entre dois espaços vetoriais com uma matriz de operadores, no caso em que os espaços $E$ e $F$ sejam escritos como soma direta de dois subespaços.

$\mathrm{Na}$ última seção veremos algumas propriedades dos espaços métricos compactos e lembraremos a definição de espaço métrico totalmente limitado. Este conceito será usado no próximo capítulo.

\subsection{Alguns resultados clássicos da análise funcional}

Dados dois espaços vetoriais $E$ e $F$ sobre o corpo $\mathbb{K}=\mathbb{R}$ ou $\mathbb{C}$, denotaremos por $L(E, F)$, ou simplesmente por $L(E)$ quando $F=E$, o espaço vetorial dos operadores lineares de $E$ a $F$. A imagem de $L \in L(E, F)$ será denotada por $\operatorname{Im} L$ e seu núcleo por Ker $L$.

Suponhamos que $E$ e $F$ sejam espaços normados. Um operador linear $T: E \rightarrow F$ é dito limitado se

$$
\sup \{\|T x\|: x \in E,\|x\|=1\}<\infty
$$


Neste caso, abusando um pouco da notação, $L(E, F)$ consistirá dos operadores lineares limitados de $E$ em $F$. O conjunto $L(E, F)$ é um espaço vetorial normado, com norma dada por

$$
\|T\|=\sup \{\|T x\|: x \in E,\|x\|=1\} \quad \text { para } T \in L(E, F) .
$$

Um resultado clássico da análise funcional diz que, se $F$ é um espaço de Banach, então $L(E, F)$ é um espaço de Banach (ver por exemplo [8], pág. 11, Proposição 1.9).

Na seguinte proposição mostraremos algumas condições necessárias e suficientes para que um operador linear definido em espaços normados seja contínuo. Em [8], pág. 10, Proposição 1.17, podemos ver uma prova deste fato.

Proposição 1.1.1. Sejam E e F dois espaços normados e $L$ um operador linear de $E$ em F. As seguintes condições são equivalentes:

i. L é contínuo.

ii. L é contínuo na origem de E.

iii. Existe $C>0$ tal que $\|L x\| \leq C\|x\|$ para todo $x \in E$.

iv. Existe $C>0$ tal que $\|L x-L y\| \leq C\|x-y\|$ para todo $x, y \in E$.

Segue-se da proposição anterior que todo operador limitado é contínuo e vice-versa. Usaremos os termos "contínuo" ou "limitado" dependendo do fato de querer marcar a continuidade ou a limitação do operador, mas com o mesmo significado.

Outro resultado clássico da análise funcional é apresentado no seguinte teorema. Ele mostra uma condição necessária e suficiente para que um espaço normado seja de Banach. Podemos ver uma prova deste fato em [8], pág. 8, Lema 1.15.

Teorema 1.1.2. Seja E um espaço normado. Então, E é um espaço de Banach se, e somente se, toda série $\sum_{k=0}^{\infty} x_{k}$ convergente em $E$ é absolutamente convergente, isto é, $\sum_{k=0}^{\infty}\left\|x_{k}\right\|$ é convergente em $\mathbb{R}$.

Definição 1.1.3. Seja $X$ um subconjunto de um espaço normado $E$. Denotaremos por $\bar{X}$ o fecho de $X$ em $E$, isto é,

$$
\bar{X}=\left\{x \in E: \text { existe }\left(x_{n}\right)_{n=1}^{\infty} \text { em } X \text { convergente a } x\right\} .
$$

Não é difícil ver que o fecho de um subespaço de $E$ é um subespaço de $E$.

Teorema 1.1.4. Sejam E um espaço normado, F um subespaço de $E$ e $G$ um espaço de Banach. Seja $L: F \rightarrow G$ um operador limitado. Existe uma única extensão de $L$ a um operador limitado $\bar{L}: \bar{F} \rightarrow G$ tal que $\|\bar{L}\|=\|L\|$. 
Podemos ver uma prova do teorema anterior em [18], pág. 75, Teorema 3.1. O operador $\bar{L}: \bar{F} \rightarrow G$ é definido como

$$
\bar{L} \bar{x}=\lim _{n \rightarrow \infty} L x_{n}
$$

onde $\left(x_{n}\right)_{n=1}^{\infty}$ é uma sequência em $F$ convergente a $\bar{x} \in \bar{F}$.

O seguinte corolário é uma consequência imediata do teorema anterior.

Corolário 1.1.5. Sejam $E_{1}$ e $E_{2}$ dois subespaços de um espaço de Banach $E$ e $L \in$ $L\left(E_{1}, E_{2}\right)$. Então, existe uma única extensão de $L$ a um operador $\bar{L} \in L\left(\bar{E}_{1}, \bar{E}_{2}\right)$ tal que $\|\bar{L}\|=\|L\|$.

Definição 1.1.6. Sejam $E$ e $F$ espaços vetoriais. Diremos que $L \in L(E, F)$ é um operador inversível (ou um isomorfismo) se é bijetor. No caso em que $E$ e $F$ sejam normados, diremos que $L$ é inversível se é bijetor, limitado e $L^{-1}$ é limitado.

A palavra "isomorfismo" serve seja no caso só vetorial ou no caso topológico. Se for claro do contexto, usaremos sempre este termo, mesmo tenha significados diferentes.

O seguinte teorema mostra que, se $E$ é um espaço de Banach e $L \in L(E)$ com $\|L\|<1$, então $I-L$ é um isomorfismo. Uma prova deste fato se pode ver em [16], pág. 375 .

Teorema 1.1.7. Seja $L \in L(E)$, onde E é um espaço de Banach. Se $\|L\|<1$, então $I-L$ é inversível e, além disso,

$$
(I-L)^{-1}=\sum_{k=0}^{\infty} L^{k}=I+L+L^{2}+\ldots,
$$

onde a série na direita é convergente na norma de $L(E)$.

Como consequência do Teorema 1.1.7 temos o seguinte corolário.

Corolário 1.1.8. Seja $L \in L(E, F)$ um operador inversível, onde $E$ e $F$ são espaços de Banach. Se $A \in L(E, F)$ e $\|A-L\|<1 /\left\|L^{-1}\right\|$, então $A$ é inversível.

Demonstração. Suponhamos que $\|A-L\|<1 /\left\|L^{-1}\right\|$. Então,

$$
\left\|L^{-1}(A-L)\right\| \leq\left\|L^{-1}\right\|\|A-L\|<\left\|L^{-1}\right\|\left(1 /\left\|L^{-1}\right\|\right)=1 .
$$

Assim, o teorema anterior implica que $I+L^{-1}(A-L)$ é inversível, onde $I$ é a identidade de $E$. Dado que $L$ é inversível, a composição

$$
L\left(I+L^{-1}(A-L)\right)=L+A-L=A
$$

também é inversível. 
Do corolário anterior temos que, se $E$ e $F$ são espaços de Banach, então o conjunto dos operadores inversíveis de $E$ em $F$, denotado por $G L(E, F)$, é um subconjunto aberto de $L(E, F)$.

Observe que, se $L$ e $T \in G(E, F)$, então

$$
L^{-1}-T^{-1}=-L^{-1}(L-T) T^{-1}
$$

De fato,

$$
L^{-1}-T^{-1}=L^{-1} L\left(L^{-1}-T^{-1}\right) T T^{-1}=L^{-1}\left(I-L T^{-1}\right) T T^{-1}=-L^{-1}(L-T) T^{-1} .
$$

Lema 1.1.9. Se E é um espaço de Banach, a aplicação

$$
\begin{aligned}
\mathcal{L}: G L(E) & \rightarrow G L(E) \\
L & \mapsto L^{-1}
\end{aligned}
$$

é contínua.

Demonstração. Tomemos $L \in G L(E)$ fixado. Seja $T \in G L(E)$ tal que $\|L-T\|<$ $1 /\left\|L^{-1}\right\|$. Assim,

$$
\left\|(L-T) L^{-1}\right\| \leq\|L-T\|\left\|L^{-1}\right\|<1 .
$$

Se segue do Teorema 1.1.7 que $I+(L-T) L^{-1}$ é inversível em $L(E)$. Além disso,

$$
\left(I+(L-T) L^{-1}\right)^{-1}=\sum_{k=0}^{\infty}\left((T-L) L^{-1}\right)^{k}
$$

Portanto,

$$
\left\|\left(I+(L-T) L^{-1}\right)^{-1}\right\| \leq \sum_{k=0}^{\infty}\left\|(T-L) L^{-1}\right\|^{k}=\frac{1}{1-\left\|(T-L) L^{-1}\right\|} \leq \frac{1}{1-\|T-L\|\left\|L^{-1}\right\|} .
$$

Dado que $T=\left(I-(L-T) L^{-1}\right) L$, então $T^{-1}=L^{-1}\left(I+(L-T) L^{-1}\right)^{-1}$. Consequentemente,

$$
\left\|T^{-1}\right\| \leq\left\|L^{-1}\right\|\left\|\left(I+(L-T) L^{-1}\right)^{-1}\right\| \leq\left\|L^{-1}\right\|\left(1-\|T-L\|\left\|L^{-1}\right\|\right)^{-1} .
$$

Daí, como $\|L-T\|\left\|L^{-1}\right\|<1$, de (1.1.1) temos

$$
\begin{aligned}
\left\|L^{-1}-T^{-1}\right\| & =\left\|-L^{-1}(L-T) T^{-1}\right\| \leq\left\|L^{-1}\right\|\|L-T\|\left\|T^{-1}\right\| \\
& \leq\left\|L^{-1}\right\|\|L-T\|\left\|L^{-1}\right\|\left(1-\|T-L\|\left\|L^{-1}\right\|\right)^{-1} \\
& <\left\|L^{-1}\right\|\|L-T\|\left\|L^{-1}\right\| .
\end{aligned}
$$

Portanto, para qualquer $\varepsilon>0$, se $T \in L(E)$ é tal que

$$
\|L-T\|<\min \left\{\varepsilon /\left\|L^{-1}\right\|^{2}, 1 /\left\|L^{-1}\right\|\right\},
$$

então $\left\|L^{-1}-T^{-1}\right\|<\varepsilon$. Este fato prova que $\mathcal{L}$ é contínua. 
Proposição 1.1.10. Sejam E um espaço de Banach e F é um espaço normado. Suponhamos que $T \in L(E, F)$ seja injetor. Então, $T^{-1}: T(E) \rightarrow E$ é contínuo se, e somente se, existe $c>0$ tal que $\|T x\| \geq c\|x\|$ para todo $x \in E$. Além disso, se $T^{-1}: T(E) \rightarrow E$ é contínuo, então $T(E)$ é um espaço de Banach.

Demonstração. Suponhamos primeiro que exista $c>0$ tal que $\|T x\| \geq c\|x\|$ para todo $x \in E$ e provemos que $T^{-1}: T(E) \rightarrow E$ é contínuo. De fato, é claro que $T^{-1}: T(E) \rightarrow E$ é bijetor. Se $y \in T(E)$, temos

$$
\|y\|=\left\|T T^{-1} y\right\| \geq c\left\|T^{-1} y\right\| .
$$

Isto é, $\left\|T^{-1} y\right\| \leq(1 / c)\|y\|$ para todo $y \in T(E)$, o que prova que $T^{-1}: T(E) \rightarrow E$ é contínuo.

Reciprocamente, se $T^{-1}: T(E) \rightarrow E$ é contínuo, existe $C>0$ tal que $\left\|T^{-1} y\right\| \leq$ $C\|y\|$ para todo $y \in T(E)$. Como $T$ é injetor, então $T^{-1} T x=x$ para todo $x \in E$. Daí,

$$
\|x\|=\left\|T^{-1} T x\right\| \leq C\|T x\| \quad \text { para todo } x \in E .
$$

Consequentemente, existe $1 / C>0$ tal que $(1 / C)\|x\| \leq\|T x\|$ para todo $x \in E$.

Agora provemos que $T(E)$ é de Banach. Seja $\left(y_{n}\right)_{n=1}^{\infty}$ uma sequência de Cauchy em $T(E)$. Então, $y_{n}=T x_{n}$ para uma sequência $\left(x_{n}\right)_{n=1}^{\infty}$ em $E$. Agora, por hipótese,

$$
\left\|x_{n}-x_{m}\right\| \leq(1 / c)\left\|T\left(x_{n}-x_{m}\right)\right\|=(1 / c)\left\|y_{n}-y_{m}\right\|
$$

Este fato implica que $\left(x_{n}\right)_{n=1}^{\infty}$ é uma sequência de Cauchy. Como $E$ é de Banach, $\left(x_{n}\right)_{n=1}^{\infty}$ converge a um $x \in E$. Portanto, da continuidade de $T$ temos que $\left(y_{n}\right)_{n=1}^{\infty}$ converge a $T(x) \in T(E)$. Logo, $T(E)$ é de Banach.

O seguinte teorema é um dos mais conhecidos de análise funcional: o Teorema da aplicação aberta. Ele será de grande importância neste trabalho.

Teorema 1.1.11 (Teorema da aplicação aberta). Sejam E, F espaços de Banach e $L: E \rightarrow F$ um operador linear contínuo sobrejetor. Então, L é aberto, isto é, para todo subconjunto aberto $\Delta$ de $E, L(\Delta)$ é aberto em $F$.

Uma consequência do Teorema da aplicação aberta é dada no seguinte corolário. A prova é imediata.

Corolário 1.1.12. Nas condições do teorema anterior, se L é injetor, então L é um isomorfismo.

O Teorema de Hahn-Banach para espaços normados, que apresentamos abaixo, será uma ferramenta de grande importância neste trabalho. Podemos ver uma prova deste teorema em [16], pág. 221, Teorema 4.3-2. 
Teorema 1.1.13 (Teorema de Hahn-Banach para espaços normados). Sejam E um espaço normado e $E_{1}$ um subespaço de $E$. Se $\varphi: E_{1} \rightarrow \mathbb{K}$ é um funcional linear limitado, existe uma extensão de $\varphi$ a um funcional linear limitado $\bar{\varphi}$ de $E$, tal que

$$
\|\bar{\varphi}\|=\|\varphi\|
$$

Neste trabalho os espaços de Hilbert separáveis têm um papel importante. Lembramos que um espaço normado $E$ é dito separável se existe uma sequência em $E$ que é densa em $E$. No resto desta seção apresentamos algumas propriedades que possuem os espaços de Hilbert separáveis.

Lembramos que um espaço normado $H$ (real ou complexo) é um espaço com produto interno se sua norma, denotada por $\|\cdot\|$, provém de um produto interno $\langle\cdot, \cdot\rangle$, isto é, se

$$
\|x\|=\sqrt{\langle x, x\rangle} \quad \text { para todo } x \in H .
$$

Um espaço de Hilbert é um espaço de Banach com produto interno.

Uma caracterização dos espaços com produto interno é apresentada no teorema seguinte. Podemos ver uma prova dele em [8], pág. 17.

Teorema 1.1.14. Suponhamos que H seja um espaço normado. Então, $H$ é um espaço com produto interno se, e somente se, sua norma satisfaz a igualdade do paralelogramo, isto é,

$$
\|x+y\|^{2}+\|x-y\|^{2}=2\|x\|^{2}+2\|y\|^{2} \quad \text { para todo } x, y \in H .
$$

No resto desta seção $H$ denotará um espaço com produto interno $\langle\cdot, \cdot\rangle$ e com norma induzida $\|\cdot\|$. Uma característica que possuem os espaços com produto interno é a bem conhecida desigualdade de Cauchy-Schwarz:

$$
|\langle x, y\rangle| \leq\|x\|\|y\| \quad \text { para todo } x, y \in H
$$

Definição 1.1.15 (Ortogonalidade). Dizemos que dois elementos $x_{1}$ e $x_{2}$ em $H$ são ortogonais se $\left\langle x_{1}, x_{2}\right\rangle=0$. Seja $X$ um subconjunto não vazio de $H$. Dizemos que $X$ é ortonormal se, para todo $x_{1}, x_{2} \in X$ com $x_{1} \neq x_{2},\left\langle x_{1}, x_{2}\right\rangle=0$ e $\langle x, x\rangle=1$ para todo $x \in X$.

Dizemos que um subconjunto ortonormal $X$ de $H$ é uma base ortonormal de $H$ se $X$ é um conjunto maximal ortonormal em $H$, isto é, para todo $y \in H \backslash X$, existe $x \in X$ tal que $\langle x, y\rangle \neq 0$. Se $X$ é uma base ortonormal de $H$ enumerável dizemos que $X$ é uma base de Hilbert de $H$.

O seguinte é um resultado clássico da álgebra linear. O método da demostração é chamado de processo de ortogonalização de Gram-Schmidt (veja-se, por exemplo, [11], pág. 278, Teorema 3). 
Teorema 1.1.16. Seja $\beta=\left\{x_{1}, x_{2}, \ldots, x_{n}\right\}$ um subconjunto linearmente independente de $H$. Então, existe um subconjunto ortonormal $\alpha=\left\{y_{1}, y_{2}, \ldots, y_{n}\right\}$ de $H$ que gera o mesmo espaço gerado por $\beta$.

Apresentaremos agora duas propriedades que possuem os espaços de Hilbert separáveis, cujas provas se podem ver, por exemplo, em [8], pág. 19, Teorema 1.36 e pág. 20, Teorema 1.38, respectivamente.

Teorema 1.1.17. Todo espaço de Hilbert separável de dimensão infinita $H$ possui uma base de Hilbert $\left(e_{n}\right)_{n=1}^{\infty}$. Além disso, se $x \in H$, então

$$
x=\sum_{i=1}^{\infty}\left\langle x, e_{i}\right\rangle e_{i} .
$$

O seguinte teorema mostra que todo espaço de Hilbert separável de dimensão infinita $H$ é linearmente isométrico a $\ell_{2}$, isto é, existe um isomorfismo $L \in L\left(H, \ell_{2}\right)$ tal que $\|L x\|=\|x\|$ para todo $x \in H$.

Teorema 1.1.18 (Riesz-Fischer). Todo espaço de Hilbert separável de dimensão infinita $H$ é isométrico $a \ell_{2}$.

\subsection{Soma, produto e quociente de espaços vetoriais e normados}

Nesta seção apresentaremos as definições da soma, produto e quociente de espaços vetoriais e algumas das suas propriedades algébricas para o caso em que os espaços não sejam normados e das propriedades topológicas para o caso em que os espaços sejam normados. Os espaços desta seção serão considerados sobre o corpo dos números reais ou complexos. Primeiro lembremos a definição da soma de dois espaços vetoriais.

Definição 1.2.1 (Soma de espaços vetoriais). Sejam $E_{1}$ e $E_{2}$ dois subespaços vetoriais de um espaço vetorial $E$. A soma dos espaços $E_{1}$ e $E_{2}$ é definida por

$$
E_{1}+E_{2}=\left\{x_{1}+x_{2}: x_{1} \in E_{1}, x_{2} \in E_{2}\right\} .
$$

Se $E_{1} \cap E_{2}=\{0\}$ dizemos que $E_{1}+E_{2}$ é uma soma direta. Neste caso denotaremos por $E_{1} \oplus E_{2}$ a soma de $E_{1}$ e $E_{2}$.

É claro que a soma de dois subespaços de um espaço $E$ é um subespaço de $E$.

Definição 1.2.2. Sejam $E_{1}$ um subespaço de um espaço normado $E$ e $x_{0} \in E$. A distância de $x_{0}$ a $E_{1}$ é definida por

$$
d\left(x_{0}, E_{1}\right)=\inf _{x \in E_{1}}\left\|x_{0}-x\right\| .
$$


Vejamos a seguinte propriedade da soma de dois subespaços de um espaço normado.

Lema 1.2.3. Seja E um espaço normado. Se $E_{1}$ é um subespaço fechado de $E$ e $E_{2}$ é de dimensão finita, então $E_{1}+E_{2}$ é fechado.

Demonstração. Demonstraremos o lema por indução sobre a dimensão de $E_{2}$. Suponhamos que $E_{2}$ tenha dimensão 1. Assim, $E_{2}=\operatorname{span}\left\{x_{0}\right\}$ para algum $x_{0} \in E$ com $\left\|x_{0}\right\|=1$. Se $x_{0} \in E_{1}$, então $E_{1}+E_{2}=E_{1}$ e portanto a soma é fechada. Suponhamos que $x_{0}$ não pertença a $E_{1}$. Seja $\left(y_{n}\right)_{n=1}^{\infty}$ uma sequência em $E_{1}+E_{2}$ convergente a $y \in E$. Então, $y_{n}=x_{n}+\lambda_{n} x_{0}$, onde $x_{n} \in E_{1}$ e $\lambda_{n} \in \mathbb{K}$ para todo $n \in \mathbb{N}$. Provemos que $\left(\lambda_{n}\right)_{n=1}^{\infty}$ é limitada. De fato, dado que $E_{1}$ é fechado, então

$$
d=d\left(x_{0}, E_{1}\right)=\inf _{x \in E_{1}}\left\|x_{0}-x\right\|>0
$$

Além disso, para $\lambda \in \mathbb{K}, \operatorname{com} \lambda \neq 0$,

$$
d\left(\lambda x_{0}, E_{1}\right)=\inf _{x \in E_{1}}\left\|\lambda x_{0}-x\right\|=\inf _{x \in E_{1}}|\lambda|\left\|x_{0}-x / \lambda\right\|=|\lambda| \inf _{y \in E_{1}}\left\|x_{0}-y\right\|=|\lambda| d .
$$

Se $\left(\lambda_{n}\right)_{n=1}^{\infty}$ não fosse limitada, então

$$
\left\|x_{n}+\lambda_{n} x_{0}\right\| \geq \inf _{x \in E_{1}}\left\|\lambda_{n} x_{0}-x\right\|=\left|\lambda_{n}\right| d
$$

isto é, $\left(y_{n}\right)_{n=1}^{\infty}$ não sería limitada. Este fato contradiz a convergência de $\left(y_{n}\right)_{n=1}^{\infty}$, que portanto é limitada.

Dado que $\left(\lambda_{n}\right)_{n=1}^{\infty}$ é limitada, ela possui uma subsequência convergente a $\lambda \in \mathbb{K}$. Consequentemente, $\left(y_{n}-\lambda_{n} x_{0}\right)_{n=1}^{\infty}=\left(x_{n}\right)_{n=1}^{\infty} \in E_{1}$ possui uma subsequência convergente a $x \in E_{1}$, pois $E_{1}$ é fechado. Assim, $\left(x_{n}+\lambda_{n} x_{0}\right)_{n=1}^{\infty}$ possui uma subsequência convergente a $x+\lambda x_{0} \in E_{1}+E_{2}$. Portanto, $y=x+\lambda x_{0} \in E_{1}+E_{2}$.

Suponhamos agora que $E_{1}+E_{1}^{\prime}$ seja fechado para qualquer subespaço $E_{1}^{\prime}$ de dimensão $n-1$. Seja $E_{2}$ um subespaço de dimensão $n$. Então, $E_{2}=E_{2}^{\prime}+\operatorname{span}\left\{x_{0}\right\}$, onde $E_{2}^{\prime}$ é um subespaço de $E_{2}$ de dimensão $n-1$ e $x_{0} \in E_{2} \backslash E_{2}^{\prime}$. Por hipótese de indução, $E_{1}+E_{2}^{\prime}$ é fechado. Pelo resultado da primeira parte da demonstração temos que

$$
E_{1}+E_{2}^{\prime}+\operatorname{span}\left\{x_{0}\right\}=E_{1}+E_{2}
$$

é fechado.

A seguinte proposição é uma consequência do Teorema de Hahn-Banach para espaços normados.

Proposição 1.2.4. Sejam E um espaço normado e $E_{1}$ um subespaço de dimensão finita. Então, $E_{1}$ tem um subespaço complementar fechado, isto é, existe um subespaço fechado $E_{2}$ de $E$ tal que $E=E_{1} \oplus E_{2}$. 
Demonstração. Seja $\left\{\alpha_{1}, \alpha_{2}, \ldots, \alpha_{n}\right\}$ uma base de $E_{1}$. Para $i=1,2, \ldots, n$, tomemos o funcional linear $\alpha_{i}^{*}: E_{1} \rightarrow \mathbb{K}$ definido por

$$
\alpha_{i}^{*}\left(\alpha_{j}\right)= \begin{cases}0 & \text { se } j \neq i \\ 1 & \text { se } j=i .\end{cases}
$$

É claro que $\alpha_{i}^{*}$ é limitado, para $i=1,2, \ldots, n$. Pelo Teorema de Hahn-Banach, podemos estender cada $\alpha_{i}^{*}$ a um funcional limitado de $E$. Podemos dar o mesmo nome a estas extensões. Seja $\alpha^{*}: E \rightarrow \mathbb{K}^{n}$ o operador linear definido por

$$
\alpha^{*}(x)=\left(\alpha_{1}^{*}(x), \alpha_{2}^{*}(x), \ldots, \alpha_{n}^{*}(x)\right) .
$$

Observe que $\alpha^{*}$ é limitado, pois os $\alpha_{i}^{*}$ são limitados. Consequentemente, Ker $\alpha^{*}$ é fechado. É fácil ver que

$$
\operatorname{Ker} \alpha^{*}=\bigcap_{i=1}^{n} \operatorname{Ker} \alpha_{i}^{*} .
$$

Daí, $E_{1} \cap \operatorname{Ker} \alpha^{*}=\{0\}$. Além disso, dado $x \in E$, existe um, e somente um, $x_{1} \in E_{1}$ tal que $\alpha^{*}(x)=\alpha^{*}\left(x_{1}\right)$. Logo, $x=x_{1}+\left(x-x_{1}\right)$, onde $x-x_{1} \in \operatorname{Ker} \alpha^{*}$. Os fatos acima mostram que

$$
E=E_{1} \oplus \operatorname{Ker} \alpha^{*},
$$

o que prova a proposição.

Definição 1.2.5. O produto cartesiano $E_{1} \times E_{2}$ de dois espaços vetoriais $E_{1}$ e $E_{2}$ é um espaço vetorial com as seguintes operações:

i. $(x, y)+(z, w)=(x+z, y+w) \quad$ para $(x, y),(z, w) \in E_{1} \times E_{2}$.

ii. $\lambda(x, y)=(\lambda x, \lambda y) \quad$ para $\lambda \in \mathbb{K} \mathrm{e}(x, y) \in E_{1} \times E_{2}$.

Se $E_{1}$ e $E_{2}$ são dois espaços normados com as normas $\|\cdot\|_{1}$ e $\|\cdot\|_{2}$, respectivamente, então o produto $E_{1} \times E_{2}$ é um espaço normado com a norma

$$
\|(x, y)\|=\sqrt{\|x\|_{1}^{2}+\|y\|_{2}^{2}} \quad \text { para }(x, y) \in E_{1} \times E_{2} .
$$

É fácil provar que, se $E_{1}$ e $E_{2}$ são de Banach, então $E_{1} \times E_{2}$ é de Banach.

O resultado seguinte é uma consequência do Teorema da aplicação aberta.

Proposição 1.2.6. Suponhamos que $E$ seja um espaço de Banach e que $E=E_{1} \oplus E_{2}$, onde $E_{1}$ e $E_{2}$ são subespaços fechados de $E$. O operador $T: E_{1} \times E_{2} \rightarrow E$ definido por $T(x, y)=x+y$ é um isomorfismo. 
Demonstração. De fato, o operador $T$ é contínuo, pois, para $(x, y) \in E_{1} \times E_{2}$,

$$
\|T(x, y)\|=\|x+y\| \leq\|x\|+\|y\| \leq \sqrt{2} \sqrt{\|x\|^{2}+\|y\|^{2}}=\sqrt{2}\|(x, y)\| .
$$

Por outro lado, dado que $E_{1}+E_{2}=E$ e $E_{1} \cap E_{2}=\{0\}$, então $T$ é bijetor. Pelo Teorema da aplicação aberta temos que $T$ é um isomorfismo.

Sejam $E$ e $F$ espaços de Banach e $L \in L(E, F)$ fixado. Suponhamos que $E=$ $E_{1} \oplus E_{2}$ e $F=F_{1} \oplus F_{2}$, onde $E_{1}$ e $E_{2}$ são subespaços fechados de $E, F_{1}$ e $F_{2}$ são subespaços fechados de $F$ e, além disso, $L\left(E_{1}\right) \subseteq F_{1}$ e $L\left(E_{2}\right) \subseteq F_{2}$. Tomemos as restrições $L_{1}=\left.L\right|_{E_{1}}: E_{1} \rightarrow F_{1}$ e $L_{2}=\left.L\right|_{E_{2}}: E_{2} \rightarrow F_{2}$ do operador $L$. Então, podemos dizer que $L$ é soma direta de $L_{1}$ e $L_{2}$, em símbolos

$$
L=L_{1} \oplus L_{1} .
$$

Por outro lado, se $E_{1}, E_{2}, F_{1}$ e $F_{2}$ são espaços de Banach e $L_{1} \in L\left(E_{1}, F_{1}\right)$ e $L_{2} \in$ $L\left(E_{2}, F_{2}\right)$, definimos o produto direto de $L_{1}$ e $L_{2}$ como o operador $\left(L_{1}, L_{2}\right): E_{1} \times E_{2} \rightarrow$ $F_{1} \times F_{2}$ dado por

$$
\left(L_{1}, L_{2}\right)(x, y)=\left(L_{1} x, L_{2} y\right)
$$

Neste caso,

$$
\begin{aligned}
\left\|\left(L_{1} x, L_{2} y\right)\right\|^{2} & =\left\|L_{1} x\right\|^{2}+\left\|L_{2} y\right\|^{2} \leq\left\|L_{1}\right\|^{2}\|x\|^{2}+\left\|L_{2}\right\|^{2}\|y\|^{2} \\
& \leq\left(\left\|L_{1}\right\|^{2}+\left\|L_{2}\right\|^{2}\right)\left(\|x\|^{2}+\|y\|^{2}\right) \\
& =\left(\left\|L_{1}\right\|^{2}+\left\|L_{2}\right\|^{2}\right)\|(x, y)\|^{2}
\end{aligned}
$$

isto é,

$$
\left\|\left(L_{1}, L_{2}\right)\right\| \leq \sqrt{\left(\left\|L_{1}\right\|^{2}+\left\|L_{2}\right\|^{2}\right)} .
$$

Este fato prova que $\left(L_{1}, L_{2}\right) \in L\left(E_{1} \times E_{2}, F_{1} \times F_{2}\right)$.

Neste trabalho também usaremos algumas propriedades do espaço quociente.

Definição 1.2.7. Sejam $E$ um espaço vetorial e $E_{1}$ um subespaço de $E$. O quociente $E / E_{1}$ é o conjunto de todos os elementos da forma

$$
\bar{x}=x+E_{1}=\left\{x+z: z \in E_{1}\right\},
$$

onde $x \in E$. É fácil ver que $E / E_{1}$ é um espaço vetorial com as operações:

i. $\bar{x}+\bar{y}=\overline{x+y} \quad$ se $x, y \in E$,

ii. $\alpha \bar{x}=\overline{\alpha x} \quad$ para $x \in E$ e $\alpha \in \mathbb{K}$.

Definição 1.2.8. A codimensão de $E_{1}$ em $E$ é a dimensão de $E / E_{1}$. 
Denotemos por $\pi: E \rightarrow E / E_{1}$ a projeção canônica de $E$ em $E / E_{1}$, isto é, $\pi(x)=$ $x+E_{1}$, para $x \in E$.

Observação 1.2.9. Observe que a codimensão de um subespaço $E_{1}$ em $E$ é finita se, e somente se, existe um subespaço de dimensão finita $E_{2}$ tal que $E=E_{1} \oplus E_{2}$. De fato, é claro que $\operatorname{Ker} \pi=E_{1}$. Seja $E_{2}$ um subespaço complementar de $E_{1}$ em $E$, isto é, $E=E_{1} \oplus E_{2}$ (este complementar existe sempre para espaços vetoriais). Tomemos uma base $\beta$ de $E_{2}$. É fácil ver que $\pi(\beta)=\{\pi(x): x \in \beta\}$ é uma base de $\pi(E)=E / E_{1}$. Consequentemente, $\operatorname{dim} E_{2}$ é finita se, e somente se, $\operatorname{dim}\left(E / E_{1}\right)$ é finita. Neste caso $\operatorname{dim} E_{2}=\operatorname{dim}\left(E / E_{1}\right)$.

Mostraremos agora duas propriedades ligadas ao conceito da codimensão de subespaços vetoriais.

Lema 1.2.10. Se $E_{1}$ e $E_{2}$ são dois subespaços de codimensão finita de um espaço vetorial $E$, então $E_{1} \cap E_{2}$ tem codimensão finita em $E$.

Demonstração. Suponhamos por contradição que o resultado não seja verdadeiro. Consideremos um complementar $E_{1}^{\prime}$ de $E_{1} \cap E_{2}$ em $E_{1}$. Assim, $E_{1}=\left(E_{1} \cap E_{2}\right) \oplus E_{1}^{\prime}$. Dado que a codimensão de $E_{1} \cap E_{2}$ é infinita e a codimensão de $E_{1}$ é finita, então a dimensão de $E_{1}^{\prime}$ é infinita. Além disso,

$$
E_{1}^{\prime} \cap E_{2}=E_{1}^{\prime} \cap\left(E_{1} \cap E_{2}\right)=\{0\} .
$$

Portanto, a soma $E_{1}^{\prime}+E_{2}$ é direta. Este fato contradiz a codimensão finita de $E_{2}$.

Lema 1.2.11. Sejam $E_{1}$ e $E_{2}$ subespaços de um espaço vetorial E. Se a codimensão de $E_{1}$ é finita e a dimensão de $E_{2}$ é infinita, então $E_{1} \cap E_{2}$ tem dimensão infinita.

Demonstração. Suponhamos por contradição que a dimensão de $E_{1} \cap E_{2}$ seja finita. Tomemos um complementar $E_{1}^{\prime}$ de $E_{1} \cap E_{2}$ em $E_{1}$. Assim,

$$
E_{1}=\left(E_{1} \cap E_{2}\right) \oplus E_{1}^{\prime} .
$$

Como a codimensão de $E_{1}$ em $E$ é finita, então a codimensão de $E_{1}^{\prime}$ em $E$ é finita. É claro que $E_{1}^{\prime} \cap E_{2}=\{0\}$. Logo, a soma $E_{1}^{\prime} \oplus E_{2}$ é direta em contradição com a dimensão infinita de $E_{2}$.

Se $E$ é um espaço normado e $E_{1}$ é um subespaço fechado de $E$, então $E / E_{1}$ se torna um espaço normado com a introdução da norma

$$
\|\bar{x}\|=\inf _{y \in \bar{x}}\|y\|=\inf _{z \in E_{1}}\|z-x\| .
$$

Não é difícil ver que (1.2.3) define uma norma para $E / E_{1}$.

O seguinte teorema mostra que, se $E$ e $E_{1}$ são espaços de Banach, então $E / E_{1}$ é também de Banach. Além disso, com estas condições, a projeção $\pi$ é contínua. Uma prova deste fato se pode ver em [7], pág. 70. 
Teorema 1.2.12. Se E é um espaço de Banach e $E_{1}$ é um subespaço fechado de E, então:

i. E/E $E_{1}$ é um espaço de Banach.

ii. $\|\pi(x)\| \leq\|x\|$ para todo $x \in E$ e assim $\pi$ é contínua.

Uma propriedade muito importante dos operadores lineares limitados é que, se a imagem de um operador limitado tem codimensão finita, então ela é fechada. Este fato é mostrado na seguinte proposição.

Proposição 1.2.13. Seja $L \in L(E, F)$, onde $E$ e $F$ são espaços de Banach. Se a codimensão da imagem de $L$ é finita, então $\operatorname{Im} L$ é um subespaço fechado de $F$.

Demonstração. De fato, do Teorema 1.2.12 se segue que $E /$ Ker $L$ é de Banach. Tome$\operatorname{mos} \bar{L}: E / \operatorname{Ker} L \rightarrow F$ dado por

$$
\bar{L}(\bar{x})=L x \quad \text { para } x \in E .
$$

Vejamos que $\bar{L}$ está bem definido. Se $\bar{x}=\bar{y}$, para $x, y \in E$, então $x-y \in \operatorname{Ker} L$. Portanto,

$$
\bar{L}(\bar{x})=L(x)=L(y)=\bar{L}(\bar{y}) .
$$

É fácil ver que $\bar{L}$ é linear. Além disso, para $x \in E$ e $x_{0} \in \operatorname{Ker} L$,

$$
\|\bar{L}(\bar{x})\|=\|L(x)\|=\left\|L\left(x-x_{0}\right)\right\| \leq\|L\|\left\|x-x_{0}\right\| .
$$

Este fato prova que

$$
\|\bar{L}(\bar{x})\| \leq\|L\| \inf _{x_{0} \in \operatorname{Ker} L}\left\|x-x_{0}\right\|=\|L\|\|\bar{x}\|,
$$

isto é, $\bar{L}$ é limitado.

É claro que $\bar{L}$ é injetor e que $\operatorname{Im} L=\operatorname{Im} \bar{L}$.

Dado que $\operatorname{Im} L$ tem codimensão finita, se segue da Observação 1.2.9 que existe um subespaço de dimensão finita $F_{1}$ de $F$ tal que $F=\operatorname{Im} L \oplus F_{1}$. Como $F_{1}$ é fechado, $F / F_{1}$ é de Banach. Consequentemente, a projeção canônica de $F$ em $F / F_{1}$, que denotamos por $\pi_{1}: F \rightarrow F / F_{1}$, é limitada. Daí, a composição

$$
\pi_{1} \bar{L}: E / \operatorname{Ker} L \rightarrow F / F_{1}
$$

é limitada.

Não é difícil provar que a restrição

$$
\left.\pi_{1}\right|_{\operatorname{Im} L}: \operatorname{Im} L \rightarrow F / F_{1}
$$


de $\pi_{1}$ a $\operatorname{Im} L$ é bijetora. Logo, como $\bar{L}$ é injetor e $\operatorname{Im} \bar{L}=\operatorname{Im} L$, a composição $\pi_{1} \bar{L}$ é bijetora. Assim, o Teorema da aplicação aberta implica que $\pi_{1} \bar{L}$ é um isomorfismo.

Consideremos agora a composição

$$
\bar{L}\left(\pi_{1} \bar{L}\right)^{-1}: F / F_{1} \rightarrow \operatorname{Im} \bar{L}=\operatorname{Im} L .
$$

Observe que

$$
\left(\left.\pi_{1}\right|_{\operatorname{Im} L}\right) \bar{L}\left(\pi_{1} \bar{L}\right)^{-1}=\left(\pi_{1} \bar{L}\right)\left(\pi_{1} \bar{L}\right)^{-1}=I_{F / F_{1}},
$$

onde $I_{F / F_{1}}$ é a identidade de $F / F_{1}$. Por outro lado, se $y \in \operatorname{Im} L$, existe $x \in E$ tal que $y=L x=\bar{L} \bar{x}$. Daí,

$$
\bar{L}\left(\pi_{1} \bar{L}\right)^{-1} \pi_{1}(y)=\bar{L}\left(\pi_{1} \bar{L}\right)^{-1}\left(\pi_{1} \bar{L}\right) \bar{x}=\bar{L} \bar{x}=y .
$$

Este fato prova que

$$
\bar{L}\left(\pi_{1} \bar{L}\right)^{-1}\left(\left.\pi_{1}\right|_{\operatorname{Im} L}\right)=I_{\operatorname{Im} L},
$$

onde $I_{\operatorname{Im} L}$ é a identidade de $\operatorname{Im} L$.

De (1.2.4) e (1.2.5) se segue que $\left.\pi_{1}\right|_{\operatorname{Im} L}: \operatorname{Im} L \rightarrow F / F_{1}$ é um isomorfismo. Em conclusão, $\operatorname{Im} L$ é de Banach sendo isomorfo a $F / F_{1}$.

\subsection{Matriz de operadores}

Na primeira parte desta seção suporemos que $E, F$ e $G$ são espaços vetoriais reais ou complexos. Lembramos que usaremos o termo isomorfismo para um operador linear $L$ entre espaços vetoriais quando $L$ for injetor e sobrejetor, enquanto se $M$ for um operador linear injetor e sobrejetor entre espaços de Banach (ou normados), o termo isomorfismo denotará que $M$ é limitado com inversa limitada. Quando os espaços $E$ e $F$ são escritos como soma direta de dois subespaços, podemos representar um operador $L \in L(E, F)$ por uma matriz de operadores os quais têm como domínio e contradomínio os subespaços das decomposições de $E$ e de $F$. Esta representação vai ser de grande utilidade em todo o trabalho, já que facilita ver o comportamento dos operadores e cada um dos subespaços considerados na soma direta de seu domínio e contradomínio. Para ver esta representação suponhamos que temos as decomposições

$$
E=E_{1} \oplus E_{2} \quad \text { e } \quad F=F_{1} \oplus F_{2} .
$$

Seja $P_{E_{1}}: E_{1} \oplus E_{2} \rightarrow E_{1}$ a projeção sobre $E_{1}$ associada à decomposição de $E$, isto é,

$$
P_{E_{1}}: E_{1} \oplus E_{2} \rightarrow E_{1}, \quad x_{1}+x_{2} \mapsto x_{1},
$$

onde $x_{1} \in E_{1}$ e $x_{2} \in E_{2}$. Assim,

$$
\operatorname{Im} P_{E_{1}}=E_{1} \quad \text { e } \quad \operatorname{Ker} P_{E_{1}}=E_{2} .
$$


De igual forma definimos as projeções $P_{E_{2}}: E_{1} \oplus E_{2} \rightarrow E_{2}, P_{F_{1}}: F_{1} \oplus F_{2} \rightarrow F_{1}$ e $P_{F_{2}}: F_{1} \oplus F_{2} \rightarrow F_{2}$.

Dado $L: E \rightarrow F$ linear, sejam $L_{i j}: E_{j} \rightarrow F_{i}$, com $i, j=1,2$, definidos por

$$
L_{i j}=\left.P_{F_{i}} L P_{E_{j}}\right|_{E_{j}} .
$$

O operador $L$ pode ser representado pela matriz de operadores

$$
L=\left(\begin{array}{ll}
L_{11} & L_{12} \\
L_{21} & L_{22}
\end{array}\right)
$$

onde a matriz age usando o produto usual de matrizes em dimensões finitas, isto é, para $x=x_{1}+x_{2} \in E$, onde $x_{1} \in E_{1}$ e $x_{2} \in E_{2}$,

$$
\left(\begin{array}{ll}
L_{11} & L_{12} \\
L_{21} & L_{22}
\end{array}\right)\left(\begin{array}{l}
x_{1} \\
x_{2}
\end{array}\right)=\left(\begin{array}{l}
L_{11} x_{1}+L_{12} x_{2} \\
L_{21} x_{1}+L_{22} x_{2}
\end{array}\right)=L_{11} x_{1}+L_{12} x_{2}+L_{21} x_{1}+L_{22} x_{2} .
$$

Definição 1.3.1. A matriz dada em (1.3.1) é chamada de matriz de operadores associada a $L$ e às decomposições de $E$ e $F$.

Suponhamos agora que temos as decomposições $E=E_{1} \oplus E_{2}, F=F_{1} \oplus F_{2}$ e $G=G_{1} \oplus G_{2}$. Consideremos os operadores $L \in L(E, F)$ e $T \in L(F, G)$, com matrizes de operadores associadas às decomposições de $E, F$ e $G$ dadas por

$$
L=\left(\begin{array}{ll}
L_{11} & L_{12} \\
L_{21} & L_{22}
\end{array}\right) \quad \text { e } \quad T=\left(\begin{array}{ll}
T_{11} & T_{12} \\
T_{21} & T_{22}
\end{array}\right)
$$

Seja $x=x_{1}+x_{2} \in E, \operatorname{com} x_{1} \in E_{1}$ e $x_{2} \in E_{2}$. Então,

$$
\begin{aligned}
T L x= & T L\left(x_{1}+x_{2}\right) \\
= & T\left(\left(L_{11} x_{1}+L_{12} x_{2}\right)+\left(L_{21} x_{1}+L_{22} x_{2}\right)\right) \\
= & T_{11}\left(L_{11} x_{1}+L_{12} x_{2}\right)+T_{12}\left(L_{21} x_{1}+L_{22} x_{2}\right) \\
& +T_{21}\left(L_{11} x_{1}+L_{12} x_{2}\right)+T_{22}\left(L_{21} x_{1}+L_{22} x_{2}\right) \\
= & T_{11} L_{11} x_{1}+T_{12} L_{21} x_{1}+T_{11} L_{12} x_{2}+T_{12} L_{22} x_{2} \\
& +T_{21} L_{11} x_{1}+T_{22} L_{21} x_{1}+T_{21} L_{12} x_{2}+T_{22} L_{22} x_{2} \\
= & \left(T_{11} L_{11}+T_{12} L_{21}\right) x_{1}+\left(T_{11} L_{12}+T_{12} L_{22}\right) x_{2} \\
& +\left(T_{21} L_{11}+T_{22} L_{21}\right) x_{1}+\left(T_{21} L_{12}+T_{22} L_{22}\right) x_{2} .
\end{aligned}
$$

Assim, a matriz da composição $T L$ associadas às decomposições de $E$ e $G$ é dada pelo produto das matrizes de $T$ e $L$, isto é,

$$
T L=\left(\begin{array}{ll}
T_{11} & T_{12} \\
T_{21} & T_{22}
\end{array}\right)\left(\begin{array}{ll}
L_{11} & L_{12} \\
L_{21} & L_{22}
\end{array}\right)=\left(\begin{array}{ll}
T_{11} L_{11}+T_{12} L_{21} & T_{11} L_{12}+T_{12} L_{22} \\
T_{21} L_{11}+T_{22} L_{21} & T_{21} L_{12}+T_{22} L_{22}
\end{array}\right) .
$$

Fazendo uso das matrizes de operadores, o seguinte lema dará uma condição suficiente para que um operador linear seja um isomorfismo. 
Lema 1.3.2. Dadas as decomposições

$$
E=E_{1} \oplus E_{2} \quad e \quad F=F_{1} \oplus F_{2}
$$

sejam $L \in L(E, F) e$

$$
L=\left(\begin{array}{cc}
L_{11} & 0 \\
L_{21} & L_{22}
\end{array}\right)
$$

a matriz de operadores de L associada às decomposições de E e F, onde 0 é o operador nulo. Se $L_{11}$ e $L_{22}$ são isomorfismos, então L é um isomorfismo.

Demonstração. Primeiro, provemos que $L$ é injetor. De fato, fixemos $x \in E$. Da descomposição de $E$, temos $x=x_{1}+x_{2}$, onde $x_{1} \in E_{1}$ e $x_{2} \in E_{2}$. Suponhamos que $L x=0$. Daí,

$$
0=L x=L_{11} x_{1}+L_{21} x_{1}+L_{22} x_{2} .
$$

Agora, $L_{11} x_{1} \in F_{1}$ e $L_{21} x_{1}+L_{22} x_{2} \in F_{2}$, assim $L_{11} x_{1}=0$ e $L_{21} x_{1}+L_{22} x_{2}=0$. Como $L_{11}$ é um isomorfismo, então $x_{1}=0$. Daí, $L_{22} x_{2}=0$. Portanto, $x_{2}=0$, pois $L_{22}$ também é um isomorfismo. Logo, $x=0$. Este fato prova que $L$ é injetor.

Por outro lado, provemos que $L$ é sobrejetor. Seja $y \in F$ fixado. Da decomposição de $F$, temos $y=y_{1}+y_{2}$, onde $y_{1} \in F_{1}$ e $y_{2} \in F_{2}$. Já que o operador $L_{11}$ é sobrejetor, existe $x_{1} \in E_{1}$ tal que $L_{11} x_{1}=y_{1}$. Daí, $L_{21} x_{1} \in F_{2}$, e como $L_{22}$ é também sobrejetor, existe $x_{2} \in E_{2}$ tal que $L_{22} x_{2}=y_{2}-L_{21} x_{1}$. Assim,

$$
L\left(x_{1}+x_{2}\right)=L_{11} x_{1}+L_{21} x_{1}+L_{22} x_{2}=y_{1}+L_{21} x_{1}+y_{2}-L_{21} x_{1}=y_{1}+y_{2}=y .
$$

Logo, $L$ é sobrejetor. Em conclusão, $L$ é um isomorfismo.

De forma análoga podemos provar que

$$
L=\left(\begin{array}{cc}
L_{11} & L_{12} \\
0 & L_{22}
\end{array}\right)
$$

é um isomorfismo se assim são $L_{11}$ e $L_{22}$.

Uma consequência imediata do lema anterior é dada no seguinte corolário.

Corolário 1.3.3. Nas condições do lema anterior, se

$$
L=\left(\begin{array}{cc}
L_{11} & 0 \\
0 & L_{22}
\end{array}\right)
$$

onde $L_{11}$ e $L_{22}$ são isomorfismos, então L é um isomorfismo. 
Observação 1.3.4. Sejam $E$ e $F$ espaços de Banach. Da Proposição 1.2.6 temos que, se $E=E_{1} \oplus E_{2}$, onde $E_{1}$ e $E_{2}$ são subespaços fechados de $E$, então $T: E_{1} \times E_{2} \rightarrow E$, definido por $T\left(x_{1}, x_{2}\right)=x_{1}+x_{2}$ é um isomorfismo. Observe que o operador $P_{E_{1}}^{\prime}$ : $E_{1} \times E_{2} \rightarrow E_{1}$ definido por $P_{E_{1}}^{\prime}\left(x_{1}, x_{2}\right)=x_{1}$ é limitado. De fato,

$$
\left\|P_{E_{1}}^{\prime}(x, y)\right\|=\|x\| \leq \sqrt{\|x\|^{2}+\|y\|^{2}}=\|(x, y)\| \quad \text { para }(x, y) \in E_{1} \times E_{2}
$$

É claro que $P_{E_{1}}=P_{E_{1}}^{\prime} T^{-1}$, o que prova que $P_{E_{1}}$ é uma projeção limitada.

Analogamente, $P_{E_{2}}, P_{F_{1}}$ e $P_{F_{2}}$ são projeções limitadas. Consequentemente, se $L \in$ $L(E, F)$, então

$$
L=\left(\begin{array}{ll}
L_{11} & L_{12} \\
L_{21} & L_{22}
\end{array}\right),
$$

onde $L_{i j}=\left.P_{F_{i}} L P_{E_{j}}\right|_{E_{j}}: E_{j} \rightarrow F_{i}$ para $i, j=1,2$, é uma matriz de operadores limitados.

\subsection{Espaços métricos compactos}

A compacidade em espaços métricos é uma ferramenta importante para a introdução dos operadores lineares compactos entre espaços de Banach, que será feita no próximo capítulo. Nesta seção veremos algumas propriedades dos espaços métricos compactos.

Definição 1.4.1. Seja $\Lambda$ um espaço topológico Hausdorff. Dizemos que $\left\{U_{\alpha}\right\}_{\alpha \in J}$ é uma cobertura aberta de $\Lambda$ se $U_{\alpha}$ é aberto para todo $\alpha$ e $\Lambda=\bigcup_{\alpha \in J} U_{\alpha}$. Uma sub-cobertura de $\left\{U_{\alpha}\right\}_{\alpha \in J}$ é uma cobertura $\left\{U_{\beta}\right\}_{\beta \in S}$ de $\Lambda$ tal que, para todo $\beta \in S, \beta=\alpha$ para algum $\alpha \in J$.

Um subconjunto $X$ de $\Lambda$ é dito compacto se toda cobertura de $X$ admite uma sub-cobertura com um número finito de elementos.

Dizemos que $X$ é completo se toda sequência de Cauchy em $X$ é convergente em $X$.

O seguinte teorema é uma caracterização dos espaços métricos compactos. Podemos ver uma prova deste resultado em [18], pág. 34, Teorema 3.8.

Teorema 1.4.2. Seja $X$ um subconjunto de um espaço métrico $M$. As seguintes condições são equivalêntes.

i. X é compacto.

ii. Toda sequência em $X$ possui uma subsequência convergente em $X$.

iii. $X$ é completo e totalmente limitado, isto é, para todo $r>0, X$ pode ser coberto por um número finito de bolas de raio $r$. 
O seguinte lema sobre espaços métricos permite provar um corolário do teorema acima.

Lema 1.4.3. Se X é um subconjunto totalmente limitado de um espaço métrico, então $\bar{X}$ é totalmente limitado.

Demonstração. Seja $r>0$ dado. Já que $X$ é totalmente limitado, existe um número finito de bolas $B\left(x_{1}, r / 2\right), B\left(x_{2}, r / 2\right), \ldots, B\left(x_{n}, r / 2\right)$, de raio $r / 2$ e centradas nos pontos $x_{1}, x_{2}, \ldots, x_{n}$, tais que

$$
X \subseteq \bigcup_{i=1}^{n} B\left(x_{i}, r / 2\right) .
$$

Assim,

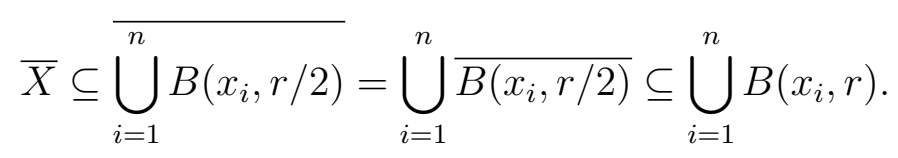

Logo, $\bar{X}$ é totalmente limitado.

Definição 1.4.4. Se diz que um subconjunto de um espaço topológico é relativamente compacto se seu fecho é compacto.

Do Lema 1.4.3 e do Teorema 1.4.2 obtemos o seguinte corolário. A prova é imediata e portanto é omitida.

Corolário 1.4.5. Todo subconjunto totalmente limitado de um espaço métrico completo é relativamente compacto.

Observação 1.4.6. Suponhamos que $X$ e $Y$ sejam subconjuntos compactos de um espaço normado $E$. Então, $X \times Y$ é um subconjunto compacto de $E \times E$. Consequentemente,

$$
X+Y=\{x+y: x \in X \text { e } y \in Y\}
$$

é compacto, sendo a imagem do operador contínuo $T: X \times Y \rightarrow X+Y$ definido por

$$
T(x, y)=x+y .
$$

Veremos agora que em um espaço normado de dimensão infinita a esfera de raio 1 com centro na origem não é compacta.

Proposição 1.4.7. Seja $F$ um subespaço fechado de um espaço vetorial normado $E$ com $F \neq E$. Seja $\varepsilon>0$ fixado. Então, existe $x \in E$, com $\|x\|=1$, tal que

$$
d(x, F)=\inf _{y \in F}\|x-y\| \geq 1-\varepsilon .
$$


Demonstração. Escolhamos $z \in E$ que não esteja em $F$. Seja $y_{0} \in F$ tal que

$$
\left\|z-y_{o}\right\| \leqslant\left(\inf _{y \in F}\|z-y\|\right)(1+\varepsilon) .
$$

Tomemos $x=\frac{z-y_{o}}{\left\|z-y_{o}\right\|}$. Então, para $y \in F$, temos

$$
\begin{aligned}
\|x-y\| & =\left\|\frac{z-y_{o}}{\left\|z-y_{o}\right\|}-y\right\|=\left\|\frac{z-y_{o}-\left\|z-y_{o}\right\| y}{\left\|z-y_{o}\right\|}\right\| \\
& =\left\|\frac{z-\left(y_{o}+\left\|z-y_{o}\right\| y\right)}{\left\|z-z_{o}\right\|}\right\| \geq\left(\inf _{y \in F}\|z-y\|\right) \frac{1}{\left\|z-y_{o}\right\|} \\
& \geq \frac{\left\|z-y_{o}\right\|}{\left\|z-y_{o}\right\|(1+\varepsilon)}=\frac{1}{1+\varepsilon} \\
& \geq 1-\varepsilon,
\end{aligned}
$$

e a prova é concluída.

Corolário 1.4.8. Seja E um espaço normado de dimensão infinita. Então, o conjunto

$$
S=\{x \in E:\|x\|=1\}
$$

não é compacto.

Demonstração. Seja $\varepsilon>0$ fixado. Tomemos $x_{1} \in E$, com $x_{1} \neq 0$, e denotemos por $F_{1}$ o espaço gerado por $x_{1}$. Dado que $F_{1}$ é fechado, da proposição anterior temos que existe $x_{2} \in E$ de norma 1 tal que $x_{2}$ não pertence a $F_{1}$ e $d\left(x_{2}, F_{1}\right) \geq 1-\varepsilon$. Seja $F_{2}$ o espaço gerado por $x_{1}$ e $x_{2}$. Assim, $F_{2}$ tem dimensão 2 e portanto é fechado. Logo, existe $x_{3} \in E$ de norma 1 tal que $x_{3}$ não pertence a $F_{2}$ e $d\left(x_{3}, F_{2}\right) \geq 1-\varepsilon$. Seja $F_{3}$ o espaço $F_{2} \oplus \operatorname{span}\left\{x_{3}\right\}$. Desta forma, construímos uma sequência de subespaços

$$
F_{1} \subseteq F_{2} \subseteq \ldots \subseteq F_{n}
$$

onde cada $F_{i}$ tem dimensão $i$. Como $E$ tem dimensão infinita, temos $F_{n} \neq E$ para $n \in \mathbb{N}$. Dado que $F_{n}$ é fechado, existe $x_{n+1} \in E$ de norma 1 tal que $x_{n+1}$ não pertence a $F_{n}$ e $d\left(x_{n+1}, F_{n}\right) \geq 1-\varepsilon$. Consequentemente, obtemos uma sequência $\left(x_{n}\right)_{n=1}^{\infty}$ em $S$ tal que $\left\|x_{n}-x_{m}\right\| \geq 1-\varepsilon$ para todo $n \neq m$. É claro que esta sequência não possui subsequência convergente. Em conclusão, $S$ não é compacto.

Por último vejamos as seguintes noções.

Definição 1.4.9. Seja $\Lambda$ um espaço topológico Hausdorff. Dizemos que uma cobertura aberta $\left\{U_{\alpha}\right\}_{\alpha \in J}$ de $\Lambda$ possui um refinamento localmente finito se existe uma cobertura $\left\{V_{\beta}\right\}_{\beta \in J^{\prime}}$ de $\Lambda$ com a propriedade que, para todo $\beta \in J^{\prime}$, existe $\alpha \in J$ tal que $V_{\beta} \subseteq U_{\alpha}$ e, para todo $x \in \Lambda$, existe uma vizinhança $B_{x}$ de $x \operatorname{com} B_{x} \cap V_{\beta} \neq \emptyset$ exceto para um número finito de índices $\beta$.

Dizemos que $\Lambda$ é paracompacto se qualquer cobertura possui um refinamento localmente finito. 
É fácil ver que todo espaço métrico compacto é paracompacto. 


\section{Capítulo 2}

\section{Operadores de Fredholm e operadores compactos}

Como foi dito na introdução, esta dissertação trata da construção do fluxo espectral para caminhos de operadores de Fredholm auto-adjuntos em espaços de Hilbert reais. Para este fim, precisamos analisar algumas das propriedades básicas dos operadores de Fredholm e, inclusive, dos operadores compactos, porque a relação entre eles é muito estrita. Algumas destas propriedades são de tipo algébrico, ou seja, concernem somente a estrutura linear e portanto podem ser dadas para operadores de Fredholm em espaços vetoriais. Outras propriedades, desta vez de tipo topológico, são mais logicamente analisadas em espaços normados ou de Banach. Os resultados apresentados servem para conhecer os operadores de Fredholm e passar no capítulo seguinte ao ambiente dos espaços de Hilbert, onde é feita a construção do fluxo espectral.

Na primeira seção deste capítulo apresentaremos várias propriedades dos operadores de Fredholm em espaços vetoriais reais que têm uma natureza só algébrica. Provaremos que a soma de um operador de Fredholm e um operador com imagem de dimensão finita é um operador de Fredholm. Mostraremos que a composição de dois operadores de Fredholm é um operador de Fredholm e que o índice é igual à soma dos índices dos dois operadores. são baseados na noção de transversalidade, que é um conceito clássico da álgebra linear e da topologia diferencial. as propriedades apresentadas são conhecidas, porém não se encontram com facilidade na literatura. Resolvemos portando providenciar diretamente uma prova delas.

Na segunda seção definiremos os operadores de Fredholm em espaços de Banach. Provaremos que a imagem de um operador de Fredholm é fechada. Os resultados mostrados para operadores de Fredholm definidos em espaços vetoriais (não necessariamente normados) continuam sendo válidos para operadores de Fredholm definidos em espaços de Banach. Mostraremos também que o conjunto dos operadores de Fredholm é um subconjunto aberto do espaço dos operadores lineares limitados e que o índice definido no conjunto dos operadores de Fredholm é uma aplicação contínua. 
Na terceira seção lembraremos a definição e algumas das principais propriedades dos operadores compactos. O resultado mais interessante que apresentaremos nesta seção é que a soma do operador identidade e um operador compacto é um operador de Fredholm de índice 0 . Como consequencia obteremos na última seção que a soma de um operador de Fredholm e um operador compacto é um operador de Fredholm.

Na última seção deste capítulo veremos uma relação que liga os operadores de Fredholm aos operadores compactos. Daremos a definição de operadores congruentes módulo operador compacto e de operador inversível modulo operador compacto. Mostraremos que um operador é de Fredholm se, e somente se, ele é inversível módulo operador compacto. Fazendo uso deste resultado, provaremos no final do capítulo que a soma de um operador de Fredholm e um operador compacto é um operador de Fredholm.

\subsection{Operadores de Fredholm em espaços vetoriais reais}

Como já dito na introdução, este trabalho trata do fluxo espectral para curvas de operadores de Fredholm auto-adjuntos em espaços de Hilbert reais. Mesmo tendo recordados no capítulo anterior vários resultados de analise funcional e álgebra linear no corpo complexo, que por sua vez serão utilizados, focamos aqui a nossa atenção sobre os operadores de Fredholm em espaços (vetoriais e de Banach) em $\mathbb{R}$. Esta escolha nos aproxima ao ponto central do nosso trabalho, ou seja, a construção do fluxo espectral.

Nesta seção $E$ e $F$ representarão espaços vetoriais reais (de dimensão finita ou infinita). O símbolo $F(E, F)$, ou $F(E)$ quando $F=E$, denotará o subespaço do $L(E, F)$ dos operadores lineares com imagem de dimensão finita.

O conúcleo de um operador $L \in L(E, F)$ é o espaço quociente $F / \operatorname{Im} L$ e será denotado por coKer $L$.

Definição 2.1.1 (Operador de Fredholm). Um operador linear $L: E \rightarrow F$ é dito de Fredholm se Ker $L$ e coKer $L$ têm dimensão finita. Neste caso, o seu índice é o inteiro

$$
\text { ind } L=\operatorname{dim} \operatorname{Ker} L-\operatorname{dim} \operatorname{coKer} L \text {. }
$$

Observação 2.1.2. Suponhamos que $E$ e $F$ sejam espaços de dimensão finita e que $L \in L(E, F)$. É claro que $L$ é um operador de Fredholm. Além disso, do Teorema do núcleo e da imagem temos

$$
\operatorname{dim} E=\operatorname{dim} \operatorname{Ker} L+\operatorname{dim} \operatorname{Im} L .
$$


Consequentemente,

$$
\text { ind } \begin{aligned}
L & =\operatorname{dim} \operatorname{Ker} L-\operatorname{dim} \operatorname{coKer} L \\
& =\operatorname{dim} E-\operatorname{dim} \operatorname{Im} L-(\operatorname{dim} F-\operatorname{dim} \operatorname{Im} L) \\
& =\operatorname{dim} E-\operatorname{dim} F .
\end{aligned}
$$

Claramente um isomorfismo é um operador de Fredholm de índice 0. Provaremos nesta seção que, se $L$ é um operador de Fredholm e $K$ é um operador com imagem de dimensão finita, então a soma $L+K$ é um operador de Fredholm do mesmo índice de $L$.

Definição 2.1.3. Dado um operador linear $L: E \rightarrow F$, um subespaço $F_{1}$ de $F$ diz-se transverso a $L$ se $\operatorname{Im} L+F_{1}=F$.

Um primeiro resultado que liga os operadores de Fredholm e a transversalidade é apresentado na seguinte proposição.

Proposição 2.1.4. Sejam $L: E \rightarrow F$ um operador de Fredholm e $F_{1}$ um subespaço de $F$ transverso a L. Então, a restrição

$$
L_{1}: L^{-1}\left(F_{1}\right) \rightarrow F_{1}
$$

de $L$ a $L^{-1}\left(F_{1}\right)$, é de Fredholm com ind $L_{1}=$ ind $L$.

Demonstração. É claro que Ker $L_{1}$ está contido em Ker $L$, pois $L_{1}$ é uma restrição de $L$. Por outro lado, se $x \in \operatorname{Ker} L$, claramente $x \in L^{-1}\left(F_{1}\right)$, portanto $\operatorname{Ker} L \subseteq \operatorname{Ker} L_{1}$. Assim,

$$
\operatorname{Ker} L=\operatorname{Ker} L_{1} \text {. }
$$

Tomemos um subespaço $E_{1}$ de $L^{-1}\left(F_{1}\right)$ tal que

$$
L^{-1}\left(F_{1}\right)=E_{1} \oplus \operatorname{Ker} L_{1}
$$

e um subespaço $F_{2}$ de $F_{1}$ tal que

$$
F_{1}=L\left(E_{1}\right) \oplus F_{2}
$$

Daí,

$$
L\left(L^{-1}\left(F_{1}\right)\right)=L\left(E_{1} \oplus \operatorname{Ker} L_{1}\right)=L\left(E_{1}\right),
$$

isto é, a imagem de $L_{1}$ é $L\left(E_{1}\right)$.

Vejamos que $F=L(E) \oplus F_{2}$. Dado que $F_{1}$ é transverso a $L$, temos que, se $y \in F$, então $y=y_{1}+y_{2}$, onde $y_{1} \in L(E)$ e $y_{2} \in F_{1}$ (esta soma pode não ser univocamente dada). Pela igualdade dada em (2.1.3), existem $z_{1} \in L\left(E_{1}\right)$ e $z_{2} \in F_{2}$ tais que $y_{2}=$ $z_{1}+z_{2}$, e assim, $y=\left(y_{1}+z_{1}\right)+z_{2}$. Como $y_{1}+z_{1} \in L(E)$ e $z_{2} \in F_{2}$, então

$$
F=L(E)+F_{2} .
$$


Agora, consideremos um elemento $v$ em $L(E) \cap F_{2}$. Seja $w \in E$ tal que $v=L w$. O subespaço $F_{2}$ está contido em $F_{1}$, logo $L w \in F_{1}$, isto é, $w \in L^{-1}\left(F_{1}\right)$. De (2.1.2) temos $w=w_{1}+w_{2}$, onde $w_{1} \in E_{1}$ e $w_{2} \in \operatorname{Ker} L_{1}$. Então,

$$
v=L w=L\left(w_{1}+w_{2}\right)=L w_{1},
$$

e portanto $v \in L\left(E_{1}\right)$. Assim, $v \in L\left(E_{1}\right) \cap F_{2}$ e de (2.1.3) segue-se $v=0$. Este fato prova que

$$
L(E) \cap F_{2}=\{0\} .
$$

Logo,

$$
F=L(E) \oplus F_{2} .
$$

A dimensão de $F_{2}$ é finita, pois $L$ é de Fredholm. As fórmulas (2.1.3) e (2.1.4) provam que

$$
\operatorname{dim} F_{2}=\operatorname{dim} \operatorname{coKer} L=\operatorname{dim} \operatorname{coKer} L_{1} .
$$

O resultado segue-se das igualdades (2.1.1) e (2.1.5).

Observação 2.1.5. Nas condições da proposição anterior segue-se claramente que, se $F_{1}$ tem dimensão finita, então $L^{-1}\left(F_{1}\right)$ tem dimensão finita. Além disso, a Observação 2.1.2 implica que o índice do operador $L$ é

$$
\text { ind } L=\operatorname{dim} L^{-1}\left(F_{1}\right)-\operatorname{dim} F_{1} \text {. }
$$

No final desta seção provaremos que a composicão de dois operadores de Fredholm é também um operador de Fredholm. No próximo lema damos a prova no caso particular das composições $T L$ e $L S$, onde $T$ e $S$ são isomorfismos e $L$ é um operador de Fredholm.

Lema 2.1.6. Sejam $E, F, G$ e $H$ espaços vetoriais. Suponhamos que $L \in L(E, F)$ seja um operador de Fredholm. Se $T \in L(F, G)$ é um isomorfismo, então $T L: E \rightarrow G$ é de Fredholm com ind $L=$ ind $T L$. Analogamente, se $S \in L(H, E)$ é um isomorfismo, então $L S: H \rightarrow F$ é de Fredholm com ind $L=$ ind $L S$.

Demonstração. Provemos a primeira parte do lema. É fácil ver que

$$
\operatorname{Ker} T L=\operatorname{Ker} L,
$$

pois $T$ é um isomorfismo.

Dado que $L$ é de Fredholm, existe um subespaço de dimensão finita $F_{2}$ de $F$ tal que

$$
F=\operatorname{Im} L \oplus F_{2} .
$$

Agora,

$$
G=T(\operatorname{Im} L) \oplus T\left(F_{2}\right)=\operatorname{Im} T L \oplus T\left(F_{2}\right) .
$$


Sendo $T$ um isomorfismo, $\operatorname{dim} T\left(F_{2}\right)=\operatorname{dim} F_{2}$. Portanto,

$$
\operatorname{dim} \operatorname{coKer} T L=\operatorname{dim} T\left(F_{2}\right)=\operatorname{dim} F_{2}=\operatorname{dim} \operatorname{coKer} L .
$$

Em conclusão, $T L$ é de Fredholm com ind $L=\operatorname{ind} T L$.

A prova da segunda parte do lema é análoga e portanto é omitida.

A seguinte proposição mostra uma caracterização dos operadores de Fredholm de índice 0 .

Proposição 2.1.7. Se $L: E \rightarrow F$ é um operador de Fredholm de índice 0, existe um operador $K$ com imagem de dimensão finita tal que $L+K$ é um isomorfismo.

Demonstração. Sejam $E_{0}$ e $F_{1}$ subespaços de $E$ e $F$, respectivamente, tais que

$$
E=E_{0} \oplus \operatorname{Ker} L \quad \text { e } \quad F=\operatorname{Im} L \oplus F_{1} .
$$

Lembrando a construção da Seção 3 do Capítulo 1, a matriz associada a $L$ e às decomposições de $E$ e $F$ é dada por

$$
L=\left(\begin{array}{cc}
L_{00} & 0 \\
0 & 0
\end{array}\right)
$$

onde $L_{00}: E_{0} \rightarrow \operatorname{Im} L$ é um isomorfismo.

Como $L$ é um operador de Fredholm de índice 0, temos

$$
\operatorname{dim} \operatorname{Ker} L=\operatorname{dim} F_{1}<\infty .
$$

Portanto, existe um isomorfismo $K^{\prime}$ de $\operatorname{Ker} L$ em $F_{1}$. Se $x \in E$, então $x=x_{0}+x_{1}$, onde $x_{0} \in E_{0}$ e $x_{1} \in \operatorname{Ker} L$. Seja $K: E \rightarrow F$ definido por $K x=K\left(x_{0}+x_{1}\right)=K^{\prime} x_{1}$. Assim,

$$
\operatorname{dim} \operatorname{Im} K=\operatorname{dim} \operatorname{Im} K^{\prime}=\operatorname{dim} F_{1}<\infty .
$$

Além disso, a matriz associada a $K$ e às decomposições de $E$ e $F$ é dada por

$$
K=\left(\begin{array}{cc}
0 & 0 \\
0 & K^{\prime}
\end{array}\right) .
$$

Consequentemente, a matriz de operadores de $L+K$ é dada por

$$
L+K=\left(\begin{array}{cc}
L_{00} & 0 \\
0 & K^{\prime}
\end{array}\right) .
$$

Dado que $L_{00}: E_{0} \rightarrow \operatorname{Im} L$ e $K^{\prime}: \operatorname{Ker} L \rightarrow F_{1}$ são isomorfismos, o Corolário 1.3 .3 prova que $L+K$ é um isomorfismo.

Provaremos agora que a soma de um operador de Fredholm $L$ e um operador $K$ com imagem de dimensão finita é um operador de Fredholm, e que, além disso, o índice de $L+K$ é igual ao índice do operador $L$. Primeiro vejamos o seguinte lema. 
Lema 2.1.8. Assuma que $E=E_{1} \oplus E_{2}$ e $F=F_{1} \oplus F_{2}$. Sejam $L: E \rightarrow F$ um operador de Fredholm e

$$
L=\left(\begin{array}{cc}
A & B \\
C & D
\end{array}\right)
$$

a matriz de $L$ associada às decomposições de E e F. Se A é invertível, então o operador $D-C A^{-1} B \in L\left(E_{2}, F_{2}\right)$ é de Fredholm $e$ ind $L=\operatorname{ind}\left(D-C A^{-1} B\right)$.

Demonstração. Seja $T: F \rightarrow F$ o operador associado à matriz

$$
T=\left(\begin{array}{cc}
I_{F_{1}} & 0 \\
-C A^{-1} & I_{F_{2}}
\end{array}\right)
$$

onde $I_{F_{1}}$ e $I_{F_{2}}$ são as identidades de $F_{1}$ e $F_{2}$, respectivamente. Do Lema 1.3 .2 temos que $T$ é um isomorfismo. Assim, segue-se do Lema 2.1.6 que $T L$ é de Fredholm e

$$
\text { ind } T L=\operatorname{ind} L .
$$

Agora,

$$
T L=\left(\begin{array}{cc}
I_{F_{1}} & 0 \\
-C A^{-1} & I_{F_{2}}
\end{array}\right)\left(\begin{array}{cc}
A & B \\
C & D
\end{array}\right)=\left(\begin{array}{cc}
A & B \\
0 & D-C A^{-1} B
\end{array}\right) .
$$

Tomemos o isomorfismo $S: E \rightarrow E$ associado à matriz

$$
S=\left(\begin{array}{cc}
I_{E_{1}} & -A^{-1} B \\
0 & I_{E_{2}}
\end{array}\right)
$$

Novamente pelo Lema 2.1.6 temos que o operador TLS é de Fredholm com

$$
\text { ind } T L S=\text { ind } T L=\text { ind } L \text {. }
$$

Fazendo a composição, obtemos

$$
T L S=\left(\begin{array}{cc}
A & B \\
0 & D-C A^{-1} B
\end{array}\right)\left(\begin{array}{cc}
I_{E_{1}} & -A^{-1} B \\
0 & I_{E_{2}}
\end{array}\right)=\left(\begin{array}{cc}
A & 0 \\
0 & D-C A^{-1} B
\end{array}\right) .
$$

Daí, $F_{2}$ é transverso a $T L S$, pois

$$
F_{1}=A\left(E_{1}\right)=(T L S)\left(E_{1}\right) \subseteq \operatorname{Im}(T L S)
$$

Provemos que $(T L S)^{-1}\left(F_{2}\right)=E_{2}$. De fato, seja $x \in E$ tal que $T L S x \in F_{2}$. Então, $x=x_{1}+x_{2}$, onde $x_{1} \in E_{1}$ e $x_{2} \in E_{2}$, e

$$
T L S\left(x_{1}+x_{2}\right)=A x_{1}+\left(D-C A^{-1} B\right) x_{2} \in F_{2} .
$$

Dado que $A x_{1} \in F_{1}$ e $\left(D-C A^{-1} B\right) x_{2} \in F_{2}$, se segue que $A x_{1}=0$. Logo, $x_{1}=0$, pois $A$ é um isomorfismo. Portanto, $x=x_{2} \in E_{2}$. Este fato prova que $(T L S)^{-1}\left(F_{2}\right)=E_{2}$.

Da Proposição 2.1.4 concluímos que

$$
\left(D-C A^{-1} B\right)=\left.T L S\right|_{E_{2}}: E_{2} \rightarrow F_{2}
$$

é um operador de Fredholm com ind $\left(D-C A^{-1} B\right)=\operatorname{ind} T L S$, o que prova o lema. 
Teorema 2.1.9. Sejam $L: E \rightarrow F$ de Fredholm e $K \in F(E, F)$. Então, o operador $L+K$ é de Fredholm $e$ ind $(L+K)=$ ind $L$.

Demonstração. Observe que a codimensão de Ker $K$ é finita. De fato, tomemos um complementar $G$ de $\operatorname{Ker} L$ em $E$. Daí, $E=G \oplus \operatorname{Ker} K$ e $\operatorname{Im} K=K(G)$ tem dimensão finita. Dado que a restrição de $K$ em $G$ é injetora e a dimensão de $K(G)$ é finita, então $\operatorname{dim} G<\infty$.

Provemos que $\operatorname{Ker}(L+K)$ é finito-dimensional. Já que $L$ e $L+K$ coincidem em Ker $K$ e Ker $L$ tem dimensão finita,

$$
\operatorname{dim}[\operatorname{Ker}(L+K) \cap \operatorname{Ker} K]=\operatorname{dim}[\operatorname{Ker} L \cap \operatorname{Ker} K]<\infty .
$$

Seja $E^{\prime}$ um complementar de $\operatorname{Ker}(L+K) \cap \operatorname{Ker} K$ em $\operatorname{Ker}(L+K)$, isto é,

$$
\operatorname{Ker}(L+K)=[\operatorname{Ker}(L+K) \cap \operatorname{Ker} K] \oplus E^{\prime} .
$$

Assim, a soma Ker $K \oplus E^{\prime}$ é direta. Consequentemente, a dimensão de $E^{\prime}$ é finita, pois a codimensão de $\operatorname{Ker} K$ em $E$ é finita. Este fato prova que

$$
\operatorname{dim} \operatorname{Ker}(L+K)<\infty .
$$

Por outro lado, provemos que $\operatorname{dim} \operatorname{coKer}(L+K)<\infty$. Claramente

$$
L(\operatorname{Ker} K)=(L+K)(\operatorname{Ker} K) \subseteq \operatorname{Im}(L+K) .
$$

Como $L$ é de Fredholm e a codimensão de $\operatorname{Ker} K$ em $E$ é finita, então $L(\operatorname{Ker} K)$ tem codimensão finita em $F$. Logo,

$$
\operatorname{dim} \operatorname{coKer}(L+K)<\infty .
$$

De (2.1.7) e (2.1.8) obtemos que $L+K$ é um operador de Fredholm.

Vejamos agora que ind $(L+K)=$ ind $L$. De fato, seja $F_{1}$ um subespaço de dimensão finita de $F$, transverso a $L$ e tal que $\operatorname{Im} K \subseteq F_{1}$. Ponhamos $E_{1}=L^{-1}\left(F_{1}\right)$. Como se viu na Observação 2.1.5, $E_{1}$ tem dimensão finita.

É claro que Ker $L$ está contido em $E_{1}$, portanto podemos escolher um subespaço $E_{2}$ de $E_{1}$ tal que $E_{1}=E_{2} \oplus \operatorname{Ker} L$. Seja $E_{0}$ um subespaço de $E$ tal que

$$
E=E_{0} \oplus E_{1}=E_{0} \oplus E_{2} \oplus \operatorname{Ker} L .
$$

Assim, sendo $L$ injetor em $E_{0} \oplus E_{2}$, temos

$$
\operatorname{Im} L=L\left(E_{0} \oplus E_{2} \oplus \operatorname{Ker} L\right)=L\left(E_{0}\right) \oplus L\left(E_{2}\right) .
$$

Demonstremos que $F=L\left(E_{0}\right) \oplus F_{1}$. Dado que $F_{1}$ é transverso a $L$ segue-se que, se $y \in F$, então $y=y_{0}+y_{1}$, onde $y_{0} \in \operatorname{Im} L$ e $y_{1} \in F_{1}$. De (2.1.9) temos que existem $z_{0} \in E_{0}$ e $z_{2} \in E_{2}$ tais que $y_{0}=L\left(z_{0}+z_{2}\right)$. Assim,

$$
y=L\left(z_{0}+z_{2}\right)+y_{1}=L z_{0}+\left(L z_{2}+y_{1}\right) .
$$


Portanto, $L z_{0} \in L\left(E_{0}\right)$, e como $L\left(E_{2}\right) \subseteq F_{1}$, então $L z_{2}+y_{1} \in F_{1}$. Este fato prova que $F=L\left(E_{0}\right)+F_{1}$.

Provemos agora que a soma $L\left(E_{0}\right)+F_{1}$ é direta. De fato, se $y \in L\left(E_{0}\right) \cap F_{1}$, então $y=L x_{0}$, para algum $x_{0}$ em $E_{0}$. Logo, $L x_{0} \in F_{1}$. Pela definição de $E_{1}$ se segue $x_{0} \in E_{1}$. Daí, temos $x_{0} \in E_{0} \cap E_{1}=\{0\}$, pois a soma $E_{0} \oplus E_{1}$ é direta. Então, $x_{0}=0$. Este fato implica que $y=0$. Assim, a soma $L\left(E_{0}\right) \oplus F_{1}$ é direta.

Consequentemente, a codimensão de $L\left(E_{0}\right)$ em $F$ é finita e é igual à dimensão de $F_{1}$.

Por outro lado, sejam

$$
L=\left(\begin{array}{ll}
L_{00} & L_{01} \\
L_{10} & L_{11}
\end{array}\right) \quad \text { e } \quad K=\left(\begin{array}{cc}
K_{00} & K_{01} \\
K_{10} & K_{11}
\end{array}\right),
$$

respectivamente, as matrizes dos operadores $L$ e $K$ associadas às decomposições

$$
E=E_{0} \oplus E_{1} \quad \text { e } \quad F=L\left(E_{0}\right) \oplus F_{1} .
$$

A imagem de $K$ está contida em $F_{1}$, portanto $K_{00}=0$ e $K_{01}=0$. Como $L\left(E_{0}\right) \cap F_{1}=$ $\{0\}$, então $L_{10}=0$. Já que $E_{1}=L^{-1}\left(F_{1}\right)$, temos $L\left(E_{1}\right) \subseteq F_{1}$. Assim, $L_{01}=0$. Logo,

$$
L=\left(\begin{array}{cc}
L_{00} & 0 \\
0 & L_{11}
\end{array}\right) \quad \text { e } \quad K=\left(\begin{array}{cc}
0 & 0 \\
K_{10} & K_{11}
\end{array}\right) .
$$

Daí,

$$
L+K=\left(\begin{array}{cc}
L_{00} & 0 \\
K_{10} & L_{11}+K_{11}
\end{array}\right) .
$$

O núcleo de $L$ está contido em $E_{1}$, assim $L_{00}: E_{0} \rightarrow L\left(E_{0}\right)$ é um isomorfismo.

Por outro lado, os subespaços $E_{1}$ e $F_{1}$ têm dimensão finita, portanto o operador $L_{11}+K_{11}: E_{1} \rightarrow F_{1}$ é de Fredholm com

$$
\operatorname{ind}\left(L_{11}+K_{11}\right)=\operatorname{dim} E_{1}-\operatorname{dim} F_{1} .
$$

Dado que $L_{00}$ é inversível, o lema anterior implica que

$$
\operatorname{ind}(L+K)=\operatorname{ind}\left(L_{11}+K_{11}\right) .
$$

Do lema anterior também temos

$$
\text { ind } L=\operatorname{ind} L_{11}=\operatorname{dim} E_{1}-\operatorname{dim} F_{1} .
$$

Consequentemente, ind $L=\operatorname{ind}(L+K)$.

Como se tinha falado acima, provaremos que a composição de dois operadores de Fredholm é também um operador de Fredholm. Além disso, se mostrará que o índice da composição é igual à soma dos índices dos dois operadores. 
Teorema 2.1.10. Sejam E, $F$ e $G$ espaços vetoriais e $L_{1}: E \rightarrow F$ e $L_{2}: F \rightarrow G$ operadores de Fredholm. A composição $L_{2} L_{1}: E \rightarrow G$ é um operador de Fredholm e

$$
\text { ind } L_{2} L_{1}=\text { ind } L_{2}+\text { ind } L_{1} \text {. }
$$

Demonstração. Dado que Ker $L_{2}$ tem dimensão finita, o espaço $F_{1}=\operatorname{Im} L_{1} \cap \operatorname{Ker} L_{2}$ tem dimensão finita. Assim, existe um subespaço de dimensão finita $F_{2}$ de $F$, tal que Ker $L_{2}=F_{1} \oplus F_{2}$. Obviamente $F_{2} \cap \operatorname{Im} L_{1}=\{0\}$.

Sejam $F_{0}$ e $F_{3}$ subespaços de $F$ tais que

$$
\operatorname{Im} L_{1}=F_{0} \oplus F_{1} \quad \text { e } \quad F=\operatorname{Im} L_{1} \oplus F_{2} \oplus F_{3} .
$$

Portanto,

$$
\operatorname{dim} \operatorname{coKer} L_{1}=\operatorname{dim} F_{2}+\operatorname{dim} F_{3} .
$$

O conúcleo de $L_{1}$ tem dimensão finita, portanto $F_{3}$ tem dimensão finita. Daí, o espaço $F_{1} \oplus F_{2} \oplus F_{3}=\operatorname{Ker} L_{2} \oplus F_{3}$ também é finito-dimensional. Dado que a soma $\operatorname{Ker} L_{2} \oplus F_{3}$ é direta, temos $F_{3} \cap \operatorname{Ker} L_{2}=\{0\}$. Logo,

$$
\operatorname{dim} L_{2}\left(F_{3}\right)=\operatorname{dim} F_{3} .
$$

Tomemos um subespaço de dimensão finita $G_{1}$ de $G$, tal que $G=\operatorname{Im} L_{2} \oplus G_{1}$. Então,

$$
\operatorname{dim} \operatorname{coKer} L_{2}=\operatorname{dim} G_{1} \text {. }
$$

Agora,

$$
\operatorname{Im} L_{2}=L_{2}(F)=L_{2}\left(F_{0} \oplus F_{1} \oplus F_{2} \oplus F_{3}\right)=L_{2}\left(F_{0}\right) \oplus L_{2}\left(F_{3}\right),
$$

assim

$$
G=L_{2}\left(F_{0}\right) \oplus L_{2}\left(F_{3}\right) \oplus G_{1} .
$$

Por outro lado, como $\operatorname{Im} L_{1}=F_{0} \oplus F_{1}$ e $F_{1}$ é subespaço de $\operatorname{Ker} L_{2}$, então

$$
\operatorname{Im} L_{2} L_{1}=L_{2}\left(\operatorname{Im} L_{1}\right)=L_{2}\left(F_{0} \oplus F_{1}\right)=L_{2}\left(F_{0}\right)
$$

Logo, a expressão (2.1.12) implica que

$$
\operatorname{dim} \operatorname{coKer}\left(L_{2} L_{1}\right)=\operatorname{dim} L_{2}\left(F_{3}\right)+\operatorname{dim} G_{1}=\operatorname{dim} F_{3}+\operatorname{dim} G_{1}<\infty,
$$

pois os subespaços $F_{3}$ e $G_{1}$ têm dimensão finita.

É claro que Ker $L_{1}$ é um subespaço de Ker $L_{2} L_{1}$. Daí, existe um subespaço $E_{0}$ de $E$ tal que

$$
\text { Ker } L_{2} L_{1}=E_{0} \oplus \operatorname{Ker} L_{1} \text {. }
$$

Mostremos que $L_{1}\left(E_{0}\right)=F_{1}$. Claramente $L_{1}\left(E_{0}\right) \subseteq \operatorname{Im} L_{1}$. O subespaço $E_{0}$ está contido em Ker $L_{2} L_{1}$, portanto, se $x \in E_{0}$, então $L_{2} L_{1} x=0$, isto é, $L_{1} x \in \operatorname{Ker} L_{2}$. Consequentemente,

$$
L_{1}\left(E_{0}\right) \subseteq \operatorname{Im} L_{1} \cap \operatorname{Ker} L_{2}=F_{1}
$$


Agora, se $x \in F_{1}$, temos $x \in \operatorname{Ker} L_{2}$ e $x=L_{1} y \in \operatorname{Im} L_{1}$ para algum $y \in E$. Logo, $x=L_{1} y \in \operatorname{Ker} L_{2}$ e assim $y \in \operatorname{Ker} L_{2} L_{1}=E_{0} \oplus \operatorname{Ker} L_{1}$. Portanto, $y=y_{1}+y_{2}$, onde $y_{1} \in E_{0}$ e $y_{2} \in \operatorname{Ker} L_{1}$. Daí,

$$
x=L_{1}\left(y_{1}+y_{2}\right)=L_{1} y_{1} \in L_{1}\left(E_{0}\right) .
$$

Então, $F_{1} \subseteq L_{1}\left(E_{0}\right)$. Consequentemente, $F_{1}=L_{1}\left(E_{0}\right)$.

Já que $E_{0} \cap \operatorname{Ker} L_{1}=\{0\}$, então

$$
\operatorname{dim} E_{0}=\operatorname{dim} L_{1}\left(E_{0}\right)=\operatorname{dim} F_{1} .
$$

Logo, de (2.1.14) temos

$$
\operatorname{dim} \operatorname{Ker}\left(L_{2} L_{1}\right)=\operatorname{dim} E_{0}+\operatorname{dim} \operatorname{Ker} L_{1}=\operatorname{dim} F_{1}+\operatorname{dim} \operatorname{Ker} L_{1}<\infty .
$$

Se segue de (2.1.13) e (2.1.15) que $L_{2} L_{1}$ é de Fredholm.

Por último, das fórmulas (2.1.10), (2.1.11), (2.1.13) e (2.1.15) temos

$$
\text { ind } \begin{aligned}
L_{2} L_{1} & =\operatorname{dim} \operatorname{Ker} L_{2} L_{1}-\operatorname{dim} \operatorname{coKer} L_{2} L_{1} \\
& =\operatorname{dim} \operatorname{Ker} L_{1}+\operatorname{dim} F_{1}-\left(\operatorname{dim} F_{3}+\operatorname{dim} G_{1}\right) \\
& =\operatorname{dim} \operatorname{Ker} L_{1}+\operatorname{dim} F_{1}-\operatorname{dim} F_{3}-\operatorname{dim} \operatorname{Ker} L_{2} \\
& =\operatorname{dim} \operatorname{Ker} L_{1}-\operatorname{dim} F_{2}-\operatorname{dim} F_{3}+\operatorname{dim} F_{1}+\operatorname{dim} F_{2}-\operatorname{dim} \operatorname{coKer} L_{2} \\
& =\operatorname{dim} \operatorname{Ker} L_{1}-\operatorname{dim} \operatorname{coKer} L_{1}+\operatorname{dim} \operatorname{Ker} L_{2}-\operatorname{dim} \operatorname{coKer} L_{2} \\
& =\operatorname{ind} L_{1}+\operatorname{ind} L_{2},
\end{aligned}
$$

e a prova é concluída.

\subsection{Operadores de Fredholm em espaços de Banach}

Nesta seção apresentaremos algumas propriedades dos operadores de Fredholm limitados entre espaços de Banach reais. Os resultados que mostraremos aqui estão diretamente relacionados com a topologia dos espaços, enquanto a seção anterior somente tomava conta dos aspectos algébricos.

Queremos destacar que, mesmo que alguns resultados desta seção sejam válidos também em espaços normados, preferimos, por razões de simplicidade, trabalhar em espaços de Banach.

No resto do capítulo, se não se diz o contrário, vamos considerar que $E$ e $F$ são espaços de Banach reais. Lembremos que neste caso $L(E, F)$ consiste dos operadores lineares limitados de $E$ em $F$. Denotaremos por $\Phi(E, F)$ o subconjunto de $L(E, F)$ dos operadores de Fredholm de $E$ em $F$. Se $n \in \mathbb{Z}$, o símbolo $\Phi_{n}(E, F)$ denotará o conjunto dos operadores de Fredholm de índice $n$. 
Observação 2.2.1. Como consequência da Proposição 1.2.13 temos que a imagem de qualquer operador de Fredholm $L \in L(E, F)$ é fechada.

A observação anterior não é sempre verdadeira se os espaços $E$ e $F$ não são completos. Um exemplo que mostra este fato é o seguinte.

Exemplo 2.2.2. Um hiperplano de um espaço vetorial $E$ é um subespaço de $E$ de codimensão 1. Seja $E_{1}$ o núcleo de um funcional linear não limitado de um espaço de Banach $E$. Então, $E_{1}$ é um hiperplano não fechado de $E$ (ver [26], pág. 139, Teorema 3.5-D). Tomemos o operador inclusão $i: E_{1} \hookrightarrow E$, isto é, $i x=x$ para todo $x \in E_{1}$. O operador $i$ é de Fredholm de índice -1 , porém, sua imagem, que é o espaço $E_{1}$, não é fechada em $E$.

Vejamos alguns exemplos de operadores de Fredholm.

Exemplo 2.2.3. Seja $\ell_{2}$ o espaço das sequências reais $\left(x_{n}\right)_{n=1}^{\infty}$ tais que $\sum_{n=1}^{\infty}\left|x_{n}\right|^{2}$ converge. Sabemos que $\ell_{2}$ é um espaço de Hilbert com produto interno dado por

$$
\langle x, y\rangle=\sum_{n=1}^{\infty} x_{n} y_{n}, \quad \text { onde } x=\left(x_{n}\right)_{n=1}^{\infty}, y=\left(y_{n}\right)_{n=1}^{\infty} \in \ell_{2} .
$$

Para um inteiro positivo $k$, definamos $T_{k} \in L\left(\ell_{2}\right)$ por

$$
T_{k}(x)=\left(x_{k+1}, x_{k+2}, \ldots\right) \quad \text { se } x=\left(x_{1}, x_{2}, \ldots .\right) .
$$

O operador $T_{k}$ é sobrejetor e seu kernel tem dimensão $k$. Assim, $T_{k}$ é um operador de Fredholm de índice $k$. Quando $k=1$ este operador é conhecido como o operador shift.

Por outro lado, tomemos $S_{k} \in L\left(\ell_{2}\right)$ definido por

$$
S_{k}\left(x_{1}, x_{2}, \ldots\right)=\left(0, \ldots, 0, x_{1}, x_{2}, \ldots\right) \quad \text { se } x=\left(x_{1}, x_{2}, \ldots\right),
$$

onde as $k$ primeiras entradas de $\left(0, \ldots, 0, x_{1}, x_{2}, \ldots\right)$ são 0 . O operador $S_{k}$ é injetor e sua imagem tem codimensão $k$. Portanto, $S_{k}$ é um operador de Fredholm de índice $-k$. Desta forma, para qualquer inteiro $k$, podemos ter um operador de Fredholm de índice $k$.

Uma das propriedades importantes do conjunto $\Phi(E, F)$ é que é aberto em $L(E, F)$. Provaremos este fato no seguinte teorema. Além disso, mostraremos que a aplicação $T \mapsto \operatorname{ind} T$ de $\Phi(E, F)$ em $\mathbb{R}$ é contínua.

Teorema 2.2.4. $O$ conjunto $\Phi(E, F)$ é aberto em $L(E, F)$ e a aplicação $T \mapsto \operatorname{ind} T$ é contínua em $\Phi(E, F)$, protanto constante em cada componente conexa. 
Demonstração. Seja $L: E \rightarrow F$ um operador de Fredholm. Desejamos provar que existe $\varepsilon>0$ tal que, se $T \in L(E, F)$ e $\|L-T\|<\varepsilon$, então $T$ é Fredholm. De fato, a Proposição 1.2.4 implica que podemos escolher um subespaço fechado $G$ de $E$ tal que

$$
E=G \oplus \operatorname{Ker} L .
$$

Como $L$ é de Fredholm, da Proposição 1.2.13 se segue que $L(G)$ é um subespaço fechado de $F$. Consequentemente, a restrição $\left.L\right|_{G}: G \rightarrow L(G)$ do operador $L$ é um isomorfismo (Teorema da aplicação aberta). Dado que a codimensão da imagem de $L$ é finita, a Observação 1.2.9 implica que

$$
F=L(G) \oplus H
$$

para algum subespaço de dimensão finita $H$ de $F$ e, além disso,

$$
\operatorname{dim} \operatorname{coKer} L=\operatorname{dim} H \text {. }
$$

Já que $F=L(G) \oplus H$, o operador $\widetilde{L}: G \times H \rightarrow L(G) \oplus H$, definido por

$$
\widetilde{L}(x, y)=L x+y,
$$

é limitado e bijetor. Como $G \times H$ e $F$ são espaços de Banach, $\widetilde{L}$ é um isomorfismo (ainda usando o Teorema da aplicação aberta).

Tomemos $T \in L(E, F)$ tal que

$$
\sup _{\substack{\|x\|=1 \\ x \in G}}\|T x-L x\|<\min \left\{1 /\left\|\widetilde{L}^{-1}\right\|, 1 /\left\|\left.L\right|_{G}{ }^{-1}\right\|\right\} .
$$

Ponhamos $\widetilde{T}: G \times H \rightarrow F$ definido por

$$
\widetilde{T}(x, y) \mapsto T x+y .
$$

Agora,

$$
\begin{aligned}
\|\widetilde{T}-\widetilde{L}\| & =\sup _{\|(x, y)\|=1}\|T x+y-L x-y\|=\sup _{\|(x, y)\|=1}\|T x-L x\| \\
& =\sup _{\substack{\|x\|=1 \\
x \in G}}\|T x-L x\|<1 /\left\|\widetilde{L}^{-1}\right\| .
\end{aligned}
$$

Portanto, do Corolário 1.1.8 temos que $\widetilde{T}$ é um isomorfismo. Logo, $F=T(G)+H$ e assim $T$ tem imagem de codimensão finita. Consequentemente, $\operatorname{Im} T$ é fechada pela Proposição 1.2.13.

Vejamos que a soma $T(G)+H$ é direta. Suponhamos que $y+z=0$, onde $y \in T(G)$ e $z \in H$. Então, $y=T x$ para algum $x \in G$ oportuno e, além disso, $\widetilde{T}(x, z)=T x+z=0$. Já que $\widetilde{T}$ é um isomorfismo, temos $x=z=0$. Assim, $y=T x=0$. Daí,

$$
F=T(G) \oplus H .
$$


Provemos que $\left.T\right|_{G}$ é injetor. De fato, seja $x \in G$ tal que $T x=0 . \operatorname{Logo}, \widetilde{T}(x, 0)=0$. Portanto, $x=0$, pois $\widetilde{T}$ é um isomorfismo. Lembrando que a codimensão de $G$ em $E$ é finita, obtemos que Ker $T$ tem dimensão finita. Dado que o conúcleo e o núcleo de $T$ é finito-dimensional, $T$ é de Fredholm.

Por último, mostremos que ind $T=\operatorname{ind} L$. Como $G$ é fechado e a dimensão de $\operatorname{Ker} T$ é finita, então $G \oplus \operatorname{Ker} T$ é fechado de codimensão finita. Escolhamos um subespaço de dimensão finita $M$ de $E$ tal que

$$
E=M \oplus G \oplus \operatorname{Ker} T .
$$

De (2.2.1) e (2.2.4) se segue

$$
\operatorname{dim} \operatorname{Ker} L=\operatorname{dim} \operatorname{Ker} T+\operatorname{dim} M .
$$

Dado que $\operatorname{Im} T=T(M \oplus G)=T(M) \oplus T(G)$ e que este espaço é fechado, pois $T$ é de Fredholm, $T$ induz um isomorfismo linear

$$
M \oplus G \rightarrow T(M) \oplus T(G) .
$$

Assim, $\operatorname{dim} M=\operatorname{dim} T(M)$. Daí, a igualdade (2.2.3) implica que

$$
\operatorname{dim} \operatorname{coKer} T=\operatorname{dim} H-\operatorname{dim} M .
$$

Das igualdades (2.2.5) e (2.2.6) segue-se

$$
\begin{aligned}
\operatorname{ind} T & =\operatorname{dim} \operatorname{Ker} T-\operatorname{dim} \operatorname{coKer} T \\
& =\operatorname{dim} \operatorname{Ker} L-\operatorname{dim} M-(\operatorname{dim} H-\operatorname{dim} M) \\
& =\operatorname{dim} \operatorname{Ker} L-\operatorname{dim} H \\
& =\operatorname{ind} L .
\end{aligned}
$$

Este fato prova que, para $T$ suficientemente perto de $L$, ind $T=$ ind $L$. Consequentemente, ind é contínua, em particular localmente constante.

Uma consequência importante do teorema anterior é dada no seguinte corolário.

Corolário 2.2.5. O conjunto $\Phi_{n}(E, F)$ é aberto em $L(E, F)$ para todo $n \in \mathbb{Z}$.

Demonstração. É claro que

$$
\Phi_{n}(E, F)=\operatorname{ind}^{-1}(\{n\}), \quad \text { onde } n \in \mathbb{Z} .
$$

Dado que a aplicação ind é contínua em $\Phi(E, F)$, temos que $\Phi_{n}(E, F)$ é aberto em $\Phi(E, F)$ para todo $n \in \mathbb{Z}$, sendo $\{n\}$ aberto em $\mathbb{Z}$. Portanto, $\Phi_{n}(E, F)$ é aberto em $L(E, F)$. 


\subsection{Operadores compactos em espaços de Banach}

Nesta seção lembraremos algumas das propriedades mais importantes dos operadores lineares compactos. Apresentaremos várias formas equivalentes de definir um operador compacto que serão usadas convenientemente para provar alguns resultados no resto do trabalho. Finalizaremos esta seção provando que, se $K \in L(E)$ é compacto, então $I-K$ é um operador de Fredholm de índice 0 , onde $I$ é a identidade de $E$. Este fato servirá para provar um resultado mais geral: A soma de um operador de Fredholm de índice $n$ e um operador compacto é um operador de Fredholm de índice $n$.

Aqui $E$ e $F$ ainda denotam espaços de Banach reais, mesmo sendo possível trabalhar em espaços normados, trabalhamos por maior simplicidade em espaço de Banach.

Definição 2.3.1. Seja $K: E \rightarrow F$ um operador linear (não necessariamente limitado). Dizemos que $K$ é compacto se leva conjuntos limitados de $E$ em conjuntos relativamente compactos de $F$, isto é, se $\overline{K(A)}$ é compacto para qualquer subconjunto limitado $A$ de $E$. Denotaremos por $K(E, F)$ o conjunto dos operadores lineares compactos de $E$ em $F$ ou simplesmente por $K(E)$ quando $F=E$.

A definição de operadores compactos pode ser analogamente dada em espaços normados não necessariamente completos.

Observação 2.3.2. Equivalentemente, podemos dizer que $K \in L(E, F)$ é compacto se leva a bola aberta $B$ de raio 1 e centro na origem de $E$ em um conjunto relativamente compacto de $F$. De fato, se $K$ é compacto, então $\overline{K(B)}$ é compacto. Reciprocamente, suponhamos que $\overline{K(B)}$ seja compacto. Se $A$ é um subconjunto limitado de $E$, então $A \subseteq B(0, r)$, onde $B(0, r)$ é uma bola aberta com centro na origem de $E$ e raio $r>0$ suficientemente grande. Dado que

$$
B=\frac{1}{r} B(0, r)=\{x / r: x \in B(0, r)\},
$$

temos

$$
\overline{K(B(0, r))}=\overline{r K(B)} .
$$

Daí, $\overline{K(B(0, r))}$ é compacto. Assim,

$$
\overline{K(A)} \subseteq \overline{K(B(0, r))}
$$

também é compacto. Este fato prova que $K$ é compacto.

Analogamente podemos provar que $K \in L(E, F)$ é compacto se, e somente se, $K$ leva a bola fechada de raio 1 e centro na origem de $E$ em um subconjunto relativamente compacto de $F$.

Como consequência da observação anterior e pela linearidade dos operadores em $L(E, F)$, temos que $K$ é compacto se, e somente se, $K(B(x, r))$ é relativamente compacto para qualquer bola aberta $B(x, r) \subseteq E$ com centro em $x \in E$ e raio $r>0$. 
Na definição de operador compacto não foi exigida a continuidade do operador $K$. Porém, devido à limitação do conjunto $K(B)$, onde $B$ é a bola com centro em $0 \in E \mathrm{e}$ raio 1 , temos que $K$ é limitado. Portanto, todo operador compacto é limitado.

Mostraremos agora outra condição suficiente e necessária para que um operador seja compacto. Este resultado é consequência do Teorema 1.4.2. Primeiro vejamos o seguinte lema que vai ser usado na demonstração.

Lema 2.3.3. Seja $\left(x_{n}\right)_{n=1}^{\infty}$ uma sequência em um espaço métrico $X$ convergente a $x$. Então, $\left(x_{n}\right)_{n=1}^{\infty}$ possui uma subsequência $\left(x_{n_{k}}\right)_{k=1}^{\infty}$ tal que

$$
\left\|x-x_{n_{k}}\right\|<2^{-k} \quad \text { para todo } k \in \mathbb{N} .
$$

Demonstração. De fato, dado que $\left(x_{n}\right)_{n=1}^{\infty}$ converge a $x$, para cada $k=1$, existe $x_{n_{1}} \in$ $\left(x_{n}\right)_{n=1}^{\infty}$ tal que $\left\|x-x_{n_{1}}\right\|<2^{-1}$. Para $k=2$, existe $x_{n_{2}} \in\left(x_{n}\right)_{n=1}^{\infty}$, com $n_{2}>n_{1}$, tal que $\left\|x-x_{n_{2}}\right\|<2^{-2}$. Desta forma obtemos uma sequência finita $\left(x_{n_{k}}\right)_{k=1}^{m}$ tal que, para $k=1,2, \ldots m, x_{n_{k}} \in\left(x_{n}\right)_{n=1}^{\infty}, n_{1}<n_{2}<\ldots<n_{m} \mathrm{e}$

$$
\left\|x-x_{n_{k}}\right\|<2^{-k} .
$$

Para $k=m+1$, existe $x_{n_{m+1}} \in\left(x_{n}\right)_{n=1}^{\infty}$, com $n_{m+1}>n_{m}$, tal que

$$
\left\|x-x_{n_{m+1}}\right\|<2^{-(m+1)} .
$$

Consequentemente, a subsequência $\left(x_{n_{k}}\right)_{k=1}^{\infty}$ construída acima é tal que

$$
\left\|x-x_{n_{k}}\right\|<2^{-k} \quad \text { para todo } k \in \mathbb{N},
$$

como queríamos provar.

Proposição 2.3.4. Um operador $K \in L(E, F)$ é compacto se, e somente se, dada uma sequência limitada $\left(x_{n}\right)_{n=1}^{\infty}$ em $E,\left(K x_{n}\right)_{n=1}^{\infty}$ possui uma subsequência convergente.

Demonstração. Seja $K \in L(E, F)$ dado. Suponhamos que $K$ seja compacto e que $\left(x_{n}\right)_{n=1}^{\infty}$ seja uma sequência limitada em $E$. Dado que $\overline{\left(K x_{n}\right)_{n=1}^{\infty}}$ é compacto, se segue do Teorema 1.4.2 que a sequência $\left(K x_{n}\right)_{n=1}^{\infty}$ possui uma subsequência convergente.

Reciprocamente, suponhamos que, para toda sequência limitada $\left(x_{n}\right)_{n=1}^{\infty}$ em $E$, a sequência $\left(K x_{n}\right)_{n=1}^{\infty}$ possua uma subsequência convergente. Sejam $A$ um subconjunto limitado de $E$ e $\left(y_{n}\right)_{n=1}^{\infty}$ uma sequência em $\overline{K(A)}$. Então, cada $y_{n}$ é o limite de uma sequência contida em $K(A)$, isto é,

$$
y_{n}=\lim _{m \rightarrow \infty} K x_{n}^{m}, \quad \text { onde } x_{n}^{m} \in A \text { para todo } n, m \in \mathbb{N} .
$$

Fixemos $n \in \mathbb{N}$. Como $\left(K x_{n}^{m}\right)_{m=1}^{\infty}$ converge a $y_{n}$ quando $m \rightarrow \infty$, escolhendo uma subsequência conveniente de $\left(K x_{n}^{m}\right)_{m=1}^{\infty}$ e dando o mesmo nome a esta subsequência, 
podemos supor, sem perda de generalidade, que $\left\|y_{n}-K x_{n}^{m}\right\|<2^{-m}$ para todo $m \in \mathbb{N}$ (este fato é consequência do lema anterior). Consequentemente, podemos supor que, para todo $n \in \mathbb{N}$,

$$
\left\|y_{n}-K x_{n}^{m}\right\|<2^{-m} \quad \text { para todo } m \in \mathbb{N} .
$$

Dado que a sequência $\left(x_{n}^{n}\right)_{n=1}^{\infty}$ é limitada, $\left(K x_{n}^{n}\right)_{n=1}^{\infty}$ possui uma subsequência convergente a algum $z \in \overline{K(A)}$. Podemos dar o mesmo nome a essa subsequência e, além disso, pelo lema anterior, supor que

$$
\left\|z-K x_{n}^{n}\right\|<2^{-n} \quad \text { para todo } n \in \mathbb{N} .
$$

Logo, de (2.3.1) e (2.3.2) temos

$$
\left\|y_{n}-z\right\|<2^{-n}+2^{-n}=2^{-n+1} \quad \text { para todo } n \in \mathbb{N} \text {. }
$$

Daí, $\left(y_{n}\right)_{n=1}^{\infty}$ possui uma subsequência convergente a $z$ em $\overline{K(A)}$. Em conclusão, $\overline{K(A)}$ é compacto e portanto $K$ é um operador compacto.

Da proposição anterior obtemos uma definição equivalente para um operador compacto. Esta nova definição vai ser de grande importância na prova de alguns resultados nesta seção.

Observação 2.3.5. Dado que todo subconjunto de um espaço de dimensão finita é compacto se, e somente se, é fechado e limitado, segue-se que, se $E$ ou $F$ são de dimensão finita, então todo operador linear limitado de $E$ em $F$ é compacto. Consequentemente, se $K$ é limitado e sua imagem tem dimensão finita, então $K$ é compacto.

Dado que a esfera de raio 1 com centro na origem de um espaço normado de dimensão infinita $E$ não é compacta (Corolário 1.4.8), temos que o operador identidade $I: E \rightarrow E$ não é um operador compacto.

O seguinte teorema mostra que $K(E, F)$ é um subespaço vetorial fechado do espaço $L(E, F)$.

Teorema 2.3.6. O conjunto $K(E, F)$ é um subespaço vetorial fechado de $L(E, F)$.

Demonstração. Denotemos por $B$ a bola aberta de raio 1 com centro em $0 \in E$.

Mostremos que a soma de dois operadores compactos é compacta. De fato, sejam $K_{1}, K_{2} \in K(E, F)$ fixados. Dado que $\overline{K_{1}(B)}$ e $\overline{K_{2}(B)}$ são subconjuntos compactos,

$$
\overline{K_{1}(B)}+\overline{K_{2}(B)}
$$

é compacto (Observação 1.4.6). Como $K_{1}(B)+K_{2}(B) \subseteq \overline{K_{1}(B)}+\overline{K_{2}(B)}$, então

$$
\overline{K_{1}(B)+K_{2}(B)} \subseteq \overline{K_{1}(B)}+\overline{K_{2}(B)},
$$


pois $\overline{K_{1}(B)}+\overline{K_{2}(B)}$ é fechado. Daí, $\overline{K_{1}(B)+K_{2}(B)}$ é um subconjunto fechado de um conjunto compacto e assim é compacto. Da Observação 2.3 .2 se segue $K_{1}+K_{2} \in$ $K(E, F)$.

Por outro lado, seja $K \in L(E, F)$ um operador compacto. Para qualquer escalar $c$, o conjunto $\overline{K(c B)}$ é compacto. Além disso,

$$
\overline{K(c B)}=\overline{(c K)(B)}
$$

portanto $\overline{(c K)(B)}$ é compacto. Da Observação 2.3.2 se segue que o operador $c K$ é compacto. Os dois fatos acima provam que $K(E, F)$ é um espaço vetorial.

Mostremos agora que $K(E, F)$ é fechado em $L(E, F)$. Seja $K$ um operador em $\overline{K(E, F)}$. desejamos provar que $K$ é compacto. Pelo Lema 1.4.3 é suficiente provar que $K(B)$ é totalmente limitado. Fixemos $r>0$. Escolhamos um $M \in K(E, F)$ tal que $\|K-M\|<r / 2$. Como $M$ é compacto, então $M(B)$ é coberto por um número finito de bolas abertas de raio $r / 2$, centradas nos pontos $y_{1}, y_{2}, \ldots, y_{n} \in F$. Para cada $x \in B$, temos

$$
\|K(x)-M(x)\|<r / 2 \quad \text { e }\left\|M(x)-y_{i}\right\|<r / 2
$$

para algum $i$. Daí, $\left\|K(x)-y_{i}\right\|<r$ para algum $i$, e portanto $K(B)$ é coberto por um número finito de bolas de raio $r$. Logo, $K \in K(E, F)$. Assim, $K(E, F)$ é um subespaço fechado de $L(E, F)$.

Na prova do teorema anterior vemos que a hipótese de que $E$ seja de Banach não é necessária para que o conjunto dos operadores compactos de $E$ em $F$ seja um espaço completo. O seguinte exemplo mostra que a completude de $F$ é necessária para que $K(E, F)$ seja de Banach.

Exemplo 2.3.7. Seja $c_{0}$ o espaço das sequências com valores em $\mathbb{R}$ que convergem a 0 . Este é um espaço de Banach com a norma

$$
\|x\|=\sup _{n}\left|x_{n}\right|, \quad \text { onde } x=\left(x_{n}\right)_{n=1}^{\infty} \in c_{0} .
$$

O subconjunto $c_{00}$ das sequências $\left(x_{n}\right)_{n=1}^{\infty} \in c_{0}$ tais que $x_{i} \neq 0$ para um número finito de índices $i$ é um subespaço de $c_{0}$. Vejamos que $c_{00}$ não é fechado. De fato, a sequência $\left(x^{n}\right)_{n=1}^{\infty}$, onde $x^{n}=(1,1 / 2, \ldots, 1 / n, 0, \ldots)$, pertence a $c_{00}$ para todo $n \in \mathbb{N}$. É fácil ver que esta sequência converge a $x=(1,1 / 2, \ldots, 1 / n, 1 /(n+1), \ldots)$ que pertence a $c_{0}$. Porém, $x$ não pertence a $c_{00}$. Portanto, $c_{00}$ não é de Banach.

Mostremos que $K\left(c_{0}, c_{00}\right)$ não é completo. A sequência de operadores $K_{n}$ definidos por

$$
K_{n} x=\left(x_{1}, x_{2} / 2, \ldots, x_{n} / n, 0, \ldots\right), \quad \text { onde } x=\left(x_{1}, x_{2}, \ldots, x_{n}, \ldots\right) \in c_{0},
$$

é uma sequência de operadores em $K\left(c_{0}, c_{00}\right)$, pois cada $K_{n}$ é limitado e tem imagem de dimensão finita. É fácil ver que esta sequência converge ao operador $K$, definido por

$$
K x=\left(x_{1}, x_{2} / 2, \ldots, x_{n} / n, x_{n+1} /(n+1), \ldots\right), \quad \text { onde } x=\left(x_{1}, x_{2}, \ldots, x_{n}, \ldots\right) \in c_{0} .
$$


É claro que $K$ não pertence a $L\left(c_{0}, c_{00}\right)$, portanto $K$ não pertence a $K\left(c_{0}, c_{00}\right)$. Assim, $K\left(c_{0}, c_{00}\right)$ não é completo.

Na Seção 2 do capítulo anterior definimos o produto direto de dois operadores limitados em espaços de Banach. Na seguinte proposição provaremos que o produto direto de dois operadores compactos é compacto.

Proposição 2.3.8. Sejam $K_{1}: E_{1} \rightarrow F_{1}$ e $K_{2}: E_{2} \rightarrow F_{2}$ operadores compactos, onde $E_{1}, F_{1}, E_{2}$ e $F_{2}$ são espaços de Banach. Então, o produto direto

$$
\left(K_{1}, K_{2}\right): E_{1} \times E_{2} \rightarrow F_{1} \times F_{2}
$$

é um operador compacto.

Demonstração. Provemos primeiro que, se $A_{1}$ e $A_{2}$ são subconjuntos limitados de $E_{1}$ e $E_{2}$, respectivamente, então $\overline{\left(K_{1}, K_{2}\right)\left(A_{1} \times A_{2}\right)}$ é compacto. De fato, dado que o produto cartesiano de dois conjuntos compactos é compacto, se segue que

$$
\overline{K_{1}\left(A_{1}\right)} \times \overline{K_{2}\left(A_{2}\right)}
$$

é compacto. Agora,

$$
\left(K_{1}, K_{2}\right)\left(A_{1} \times A_{2}\right)=K_{1}\left(A_{1}\right) \times K_{2}\left(A_{2}\right) \subseteq \overline{K_{1}\left(A_{1}\right)} \times \overline{K_{2}\left(A_{2}\right)} .
$$

Consequentemente,

$$
\overline{\left(K_{1}, K_{2}\right)\left(A_{1} \times A_{2}\right)} \subseteq \overline{K_{1}\left(A_{1}\right)} \times \overline{K_{2}\left(A_{2}\right)} .
$$

Daí, $\overline{\left(K_{1}, K_{2}\right)\left(A_{1} \times A_{2}\right)}$ é compacto.

Por outro lado, é claro que, se $A$ é um subconjunto limitado de $E_{1} \times E_{2}$, existem subconjuntos limitados $C_{1}$ e $C_{2}$ de $E_{1}$ e $E_{2}$, respectivamente, tais que $A \subseteq C_{1} \times C_{2}$. Logo,

$$
\overline{\left(K_{1}, K_{2}\right)(A)} \subseteq \overline{\left(K_{1}, K_{2}\right)\left(C_{1} \times C_{2}\right)} .
$$

Como se provou acima, $\overline{\left(K_{1}, K_{2}\right)\left(C_{1} \times C_{2}\right)}$ é compacto, portanto $\overline{\left(K_{1}, K_{2}\right)(A)}$ é compacto. Em conclusão, $\left(K_{1}, K_{2}\right)$ é um operador compacto.

Observação 2.3.9. Suponhamos que $K: E \rightarrow F$ seja compacto. Observe que, se $E_{1}$ é um subespaço qualquer de $E$ e $\overline{K\left(E_{1}\right)} \subseteq F_{1}$ para algum subespaço $F_{1}$ de $F$, então a restrição

$$
\left.K\right|_{E_{1}}: E_{1} \rightarrow F_{1}
$$

é também um operador compacto. De fato, seja $A_{1}$ um subconjunto limitado de $E_{1}$. Como $\overline{K\left(E_{1}\right)} \subseteq F_{1}$, então

$$
\overline{K\left(A_{1}\right)}=\overline{K\left(A_{1}\right)} \cap F_{1},
$$

isto é, o fecho de $K\left(A_{1}\right)$ em $F_{1}$ e em $F$ coincidem (observe que não estamos supondo $F_{1}$ fechado em $\left.F\right)$. Dado que $K$ é compacto, temos que $\overline{K\left(A_{1}\right)}$ é compacto. Consequentemente, a restrição $\left.K\right|_{E_{1}}$ é compacta. 
Uma outra propriedade importante dos operadores compactos é que a composição de um operador compacto e um operador limitado é um operador compacto. Este fato é provado no seguinte teorema.

Teorema 2.3.10. Sejam E, F, $G$ e $H$ espaços de Banach, e $T: E \rightarrow F, K: F \rightarrow G$, $S: G \rightarrow H$ operadores limitados. Se $K$ é compacto, então $K T$ e $S K$ são compactos.

Demonstração. Se $C$ é um subconjunto limitado de $E$, então $T(C) \subseteq F$ é limitado, pois $T$ é limitado. Assim, $\overline{K(T(C))}$ é compacto. Daí, $K T$ é compacto.

Por outro lado, seja $A$ um subconjunto limitado de $F$. Dado que $S$ é contínuo e $\overline{K(A)}$ é compacto, então $S(\overline{K(A)})$ é compacto. Agora, $S(K(A)) \subseteq S(\overline{K(A)})$ que é fechado, portanto $\overline{S(K(A))} \subseteq S(\overline{K(A)})$. Logo, $\overline{S(K(A))}$ é compacto, pois é um subconjunto fechado de um compacto. Assim, $S K$ é compacto.

Observe que $L(E)$ tem estrutura de anel com as operações soma e composição de operadores. O teorema acima mostra em particular que $K(E)$ é um ideal à esquerda e à direita de $L(E)$.

Na primeira seção deste capítulo se provou que a soma de um operador de Fredholm $L$ e um operador $K$ com imagem de dimensão finita é também um operador de Fredholm (no sentido algébrico). No final deste capítulo mostraremos que, se $L$ é de Fredholm (no sentido topológico) e $K$ é compacto, então $L+K$ também é de Fredholm com o mesmo índice de $L$. Vejamos primeiro que $I-K$ é um operador de Fredholm e que $\operatorname{ind}(I-K)=\operatorname{ind} I=0$.

Teorema 2.3.11. Se $K: E \rightarrow E$ é um operador compacto, então $I-K$ é um operador de Fredholm e, além disso,

$$
\operatorname{ind}(I-K)=\operatorname{ind} I=0 .
$$

Demonstração. Lembremos que o operador identidade $I$ de um espaço normado $F$ é compacto se, e somente se, $F$ tem dimensão finita. Agora, se $x \in \operatorname{Ker}(I-K)$, então

$$
I x=I x-(I-K) x=I x-I x+K x=K x,
$$

isto é, a identidade restrita ao kernel de $I-K$ coincide com $\left.K\right|_{\operatorname{Ker}(I-K)}$. Da Observação 2.3.9 temos que $\left.K\right|_{\operatorname{Ker}(I-K)}$ é compacto. Daí, $\left.I\right|_{\operatorname{Ker}(I-K)}$ é um operador compacto. Consequentemente, $\operatorname{Ker}(I-K)$ tem dimensão finita.

Mostremos agora que a imagem de $I-K$ é fechada. Seja $G$ um complementar fechado de $\operatorname{Ker}(I-K)$, isto é, $E=G \oplus \operatorname{Ker}(I-K)$. Tomemos os operadores limitados

$$
\left.(I-K)\right|_{G}: G \rightarrow E \quad \text { e }\left.\quad K\right|_{G}: G \rightarrow E,
$$


que são as restrições a $G$ de $I-K$ e $K$, respectivamente. É claro que $\left.(I-K)\right|_{G}$ é injetor e que $\operatorname{Im}(I-K)=(I-K)(G)$. Se segue da Proposição 1.1.10 que para provar que a imagem de $I-K$ é fechada é suficiente provar que o operador inverso

$$
\left.(I-K)\right|_{G} ^{-1}:(I-K)(G) \rightarrow G
$$

é limitado, e para este fim, é suficiente provar que $\left.(I-K)\right|_{G} ^{-1}$ é contínuo em 0 pela Proposição 1.1.1. De fato, suponhamos por absurdo que $\left.(I-K)\right|_{G} ^{-1}$ não seja contínuo em 0. Logo, existe uma sequência $\left(x_{n}\right)_{n=1}^{\infty}$ em $G$ não convergente a 0 e tal que $(I-$ $K) x_{n} \rightarrow 0$. Escolhendo uma subsequência (conveniente) podemos assumir, sem perda de generalidade, que existe $r>0$ tal que $\left\|x_{n}\right\| \geq r$ para todo $n$. Então, $1 /\left\|x_{n}\right\| \leqslant 1 / r$ para todo $n$, e assim $(I-K)\left(x_{n} /\left\|x_{n}\right\|\right) \rightarrow 0$. Além disso, $x_{n} /\left\|x_{n}\right\|$ tem módulo 1 , logo $\left(K\left(x_{n} /\left\|x_{n}\right\|\right)\right)_{n=1}^{\infty}$ possui uma subsequência convergente, pois $K$ é compacto. Já que

$$
(I-K)\left(\frac{x_{n}}{\left\|x_{n}\right\|}\right)=\frac{x_{n}}{\left\|x_{n}\right\|}-K\left(\frac{x_{n}}{\left\|x_{n}\right\|}\right)
$$

e $G$ é fechado, segue-se que uma subsequência de $\left(x_{n} /\left\|x_{n}\right\|\right)_{n=1}^{\infty}$ converge a algum elemento $z \in G$. É claro que $\|z\|=1$. Consequentemente, $0=z-K(z)=(I-K)(z)$, contradizendo o fato que $G \cap \operatorname{Ker}(I-K)=\{0\}$. Portanto, $(I-K)(E)=(I-K)(G)$ é fechado.

Provemos que $(I-K)(E)$ tem codimensão finita. Suponhamos por absurdo que (I$K)(E)$ não tenha codimensão finita. Como consequência do Lema 1.2.3, adicionando um subespaço de dimensão 1 a $(I-K)(E)$ indutivamente, obtemos uma sequência de subespaços fechados

$$
(I-K)(E)=H_{0} \subseteq H_{1} \subseteq \ldots \subseteq H_{n} \subseteq \ldots,
$$

tais que cada $H_{n}$ é fechado e tem codimensão $1 \mathrm{em} H_{n+1}$. Pela Proposição 1.4.7, em cada $H_{n}$ existe um elemento $x_{n}$ tal que $\left\|x_{n}\right\|=1 \mathrm{e}\left\|x_{n}-y\right\| \geq 1-\varepsilon$ para todo $y \in H_{n-1}$. Então, para todo $k<n$,

$$
\left\|K x_{n}-K x_{k}\right\|=\left\|x_{n}-(I-K) x_{n}-x_{k}+(I-K) x_{k}\right\| \geq 1-\varepsilon,
$$

pois $x_{n} \in H_{n} \mathrm{e}-(I-K) x_{n}-x_{k}+(I-K) x_{k} \in H_{n-1}$. Este fato prova que a sequência $\left(K x_{n}\right)_{n=1}^{\infty}$ não tem subsequência convergente, contradizendo a compacidade de $K$. Daí, a codimensão da imagem de $I-K$ é finita. Assim, $I-K$ é de Fredholm.

Por último, vejamos que ind $(I-K)=0$. Claramente, para cada $t \in \mathbb{R}$, o operador $t K$ é compacto. A aplicação $t \mapsto t K$ é contínua. Logo, a aplicação $t \mapsto \operatorname{ind}(I-t K)$ também é contínua. Dado que $I-t K$ pertence a $\Phi(E)$ para todo $t \in \mathbb{R}$, se segue do Teorema 2.2.4 que esta aplicação é constante. Tomando $t=0$ e $t=1$, concluímos que

$$
\operatorname{ind}(I-K)=\operatorname{ind} I=0 \text {, }
$$

isto é, $I-K$ é um operador de Fredholm de índice 0. 


\subsection{Operadores congruentes módulo operador com- pacto}

Veremos nesta seção uma relação de grande importância que liga os operadores compactos aos operadores de Fredholm. Esta relação nos permitirá provar facilmente que a soma de um operador compacto e um operador de Fredholm é um operador de Fredholm.

Definição 2.4.1. Sejam $L, T \in L(E, F)$ dados. Dizemos que $L$ e $T$ são congruentes módulo operador compacto se $L-T$ é compacto, e escrevemos $L \cong T$.

Em alguns livros os operadores congruentes módulo operador compacto são chamados de Calkin equivalentes.

Vejamos que a congruência $\cong$ é uma relação de equivalência. De fato, sejam $L, T, S \in L(E, F)$. Esta congruência é reflexiva, pois claramente $L \cong L$. Agora, se $L \cong T$, então $L-T$ é compacto. Assim, $T-L=-(L-T)$ é compacto. Daí, $T \cong L$, o que prova que a congruência é simétrica. Por último vejamos que ela é transitiva. Se $L \cong T$ e $T \cong S$, então $L-T$ e $T-S$ são operadores compactos. Como a soma de operadores compactos é compacta, temos que

$$
L-T+T-S=L-S
$$

é compacto, isto é, $L \cong S$.

Outra propriedade dos operadores congruentes módulo operador compacto é apresentada na seguinte proposição.

Proposição 2.4.2. Sejam $L, T \in L(E, F)$ e $L_{1}, T_{1} \in L(G, E)$, onde $E, F$ e $G$ são espaços de Banach. Suponhamos que $L \cong T$ e $L_{1} \cong T_{1}$. Então, $L L_{1} \cong T T_{1}$.

Demonstração. De fato, como $L-T$ e $L_{1}-T_{1}$ são compactos, se segue do Teorema 2.3.10 que $(L-T) L_{1}$ e $T\left(L_{1}-T_{1}\right)$ são compactos. A soma de operadores compactos é compacta, portanto

$$
(L-T) L_{1}+T\left(L_{1}-T_{1}\right)
$$

é compacto. Assim,

$$
L L_{1}-T T_{1}=(L-T) L_{1}+T\left(L_{1}-T_{1}\right)
$$

é compacto, o que prova a proposição.

Observe que, se $L \cong T$ e $L_{1} \cong T_{1}$, onde $L, T, L_{1}, T_{1} \in L(E, F)$, então

$$
L+L_{1} \cong\left(T+T_{1}\right)
$$

Este fato se deve a que a soma de operadores compactos é compacta. 
Dizemos que $L: E \rightarrow F$ é inversível módulo operador compacto se existe um operador limitado $L_{1}: F \rightarrow E$ tal que

$$
L_{1} L \cong I_{E} \quad \text { e } \quad L L_{1} \cong I_{F},
$$

onde $I_{E}$ e $I_{F}$ denotam as identidades de $E$ e $F$, respectivamente. Neste caso dizemos que $L_{1}$ é uma inversa de $L$ módulo operador compacto.

Observação 2.4.3. Suponhamos que, para $L \in L(E, F)$, existam $L_{1}, L_{2} \in L(F, E)$ tais que

$$
L L_{1} \cong I_{F} \quad \text { e } \quad L_{2} L \cong I_{E} .
$$

Vejamos que $L_{1} \cong L_{2}$ e que, além disso, $L_{1}$ e $L_{2}$ são inversas de $L$ módulo operador compacto. De fato, por hipótese,

$$
K_{1}=L L_{1}-I_{F} \quad \text { e } \quad K_{2}=L_{2} L-I_{E}
$$

são compactos. Assim, temos as relações seguintes

$$
\begin{aligned}
L L_{1}-I_{F} & =K_{1} \\
L_{2} L L_{1}-L_{2} & =L_{2} K_{1} \\
\left(K_{2}+I_{E}\right) L_{1}-L_{2} & =L_{2} K_{1} \\
K_{2} L_{1}+L_{1}-L_{2} & =L_{2} K_{1} \\
L_{1}-L_{2} & =L_{2} K_{1}-K_{2} L_{1} .
\end{aligned}
$$

Este fato prova que $K_{1,2}=L_{1}-L_{2}$ é compacto.

Agora, vejamos que $L_{2}$ é uma inversa de $L$ módulo operador compacto. Dado que $L_{1}=L_{2}+K_{1,2}$, onde $K_{1,2}$ é compacto, de (2.4.1) temos

$$
L L_{2}-I_{F}=K_{1}-L K_{1,2} .
$$

Se segue do Teorema 2.3.10 que $L K_{1,2}$ é compacto. Logo, $L L_{2}-I_{F}=K_{1}-L K_{1,2}$ é compacto, pois a soma de operadores compactos é compacta. Consequentemente, $L L_{2}-I_{F}$ e $L_{2} L-I_{E}$ são compactos. Em conclusão, $L_{2}$ é uma inversa de $L$ módulo operador compacto.

Analogamente, $L_{1}$ é uma inversa de $L$ módulo operador compacto.

No próximo teorema mostraremos que $L$ é um operador de Fredholm se, e somente se, $L$ é inversível módulo operador compacto.

Teorema 2.4.4. Um operador $L \in L(E, F)$ é de Fredholm se, e somente se, é inversivel módulo operadores compactos. Podemos escolher uma inversa de L módulo operadores compactos que tenha imagem de codimensão finita. 
Demonstração. Suponhamos primeiro que $L \in L(E, F)$ seja um operador de Fredholm e provemos que existe $S \in L(F, E)$ tal que $I_{E}-S L$ e $I_{F}-L S$ são operadores compactos. Expressemos os espaços $E$ e $F$ nas seguintes somas diretas

$$
E=G \oplus \operatorname{Ker} L \quad \text { e } \quad F=\operatorname{Im} L \oplus H,
$$

onde $G$ e $H$ são subespaços fechados de $E$ e $F$, respectivamente. Assim, $L^{-1}: \operatorname{Im} L \rightarrow G$ existe e é limitado pelo Teorema da aplicação aberta. Tomemos $P: \operatorname{Im} L \oplus H \rightarrow \operatorname{Im} L$ a projeção sobre $\operatorname{Im} L$ e $i: G \hookrightarrow G \oplus \operatorname{Ker} L$ a inclusão. Seja $S \in L(F, E)$ a composição $i L^{-1} P$.

Agora, se $x+y \in \operatorname{Im} L \oplus H, \operatorname{com} x \in \operatorname{Im} L$ e $y \in H$, então

$$
\begin{aligned}
\left(I_{F}-L S\right)(x+y) & =I_{F}(x+y)-L\left(i L^{-1} P\right)(x+y) \\
& =x+y-L i L^{-1} x \\
& =x+y-x \\
& =y .
\end{aligned}
$$

Este fato prova que $I_{F}-L S$ é a projeção sobre $H$. Além disso,

$$
\operatorname{dim} \operatorname{Im}\left(I_{F}-L S\right)=\operatorname{dim} H=\operatorname{coKer} L<\infty,
$$

isto é, $I_{F}-L S$ é compacto.

Por outro lado, se $x+y \in G \oplus \operatorname{Ker} L$, onde $x \in G$ e $y \in \operatorname{Ker} L$, temos

$$
\begin{aligned}
\left(I_{E}-S L\right)(x+y) & =I_{E}(x+y)-i L^{-1} P L(x+y) \\
& =x+y-i L^{-1} P L x \\
& =x+y-L^{-1} L x \\
& =x+y-x \\
& =y
\end{aligned}
$$

isto é, $I_{E}-S L$ é a projeção sobre Ker $L$. Dado que

$$
\operatorname{dim} \operatorname{Im}\left(I_{E}-S L\right)=\operatorname{dim} \operatorname{Ker} L<\infty,
$$

$I_{E}-S L$ é compacto.

Os fatos acima mostram que $S$ é uma inversa de $L$ módulo operador compacto.

Reciprocamente, suponhamos que $T \in L(F, E)$ seja uma inversa de $L$ módulo operadores compactos. Assim, $K=I_{E}-T L$ é compacto. Portanto, $T L=I_{E}-K$ é de Fredholm. Este fato prova que $\operatorname{Ker}(T L)$ tem dimensão finita. Dado que $\operatorname{Ker} L \subseteq$ $\operatorname{Ker}(T L)$, temos $\operatorname{dim} \operatorname{Ker} L<\infty$. Analogamente, $L T$ é de Fredholm. Se segue da Proposição 1.2.13 que $L T$ tem imagem fechada de codimensão finita. Além disso, $\operatorname{Im}(L T) \subseteq \operatorname{Im} L$. Logo, $\operatorname{Im} L$ é fechado e tem codimensão finita. Em conclusão, $L$ é de Fredholm. 
Terminamos o capítulo mostrando que a soma de um operador compacto e um operador de Fredholm é também um operador de Fredholm, estendendo o Teorema 2.1.9.

Teorema 2.4.5. Se $L \in L(E, F)$ é um operador de Fredholm e $K \in L(E, F)$ é um operador compacto, então $L+K$ é de Fredholm. Além disso,

$$
\operatorname{ind}(L+K)=\operatorname{ind} L .
$$

Demonstração. Sejam $L \in \Phi(E, F)$ e $K \in K(E, F)$ fixados. O Teorema 2.4.4 implica que existem $L_{1} \in L(F, E), K_{1} \in K(F, F)$ e $K_{2} \in K(E, E)$ tais que

$$
L L_{1}-I_{F}=K_{1} \quad \text { e } \quad L_{1} L-I_{E}=K_{2} .
$$

Assim,

$$
L L_{1}+K L_{1}-I_{F}=K_{1}+K L_{1} \quad \text { e } \quad L_{1} K+L_{1} L-I_{E}=K_{2}+L_{1} K .
$$

Daí,

$$
(L+K) L_{1}-I_{F}=K_{1}+K L_{1} \quad \text { e } \quad L_{1}(L+K)-I_{E}=K_{2}+L_{1} K .
$$

Dado que $K_{1}+K L_{1}$ e $K_{2}+L_{1} K$ são compactos, se segue de (2.4.2) que o operador $L_{1}$ é uma inversa de $L+K$ módulo operador compacto. Portanto, pelo Teorema 2.4.4 $L+K$ é de Fredholm.

Por outro lado, $t K$ é um operador compacto para todo $t \in \mathbb{R}$. Assim, $L+t K$ é de Fredholm para $0 \leqslant t \leqslant 1$. Dado que a aplicação ind é contínua, temos

$$
\operatorname{ind}(L+K)=\operatorname{ind}(L+t K)=\operatorname{ind} L,
$$

e o teorema é provado. 


\section{Capítulo 3}

\section{Operadores de Fredholm auto-adjuntos em espaços de Hilbert}

O propósito deste capítulo é apresentar algumas das propriedades dos operadores de Fredholm em espaços de Hilbert. Para este fim, nas duas primeiras seções lembraremos alguns resultados conhecidos da análise funcional dos espaços de Hilbert, assim como a definição do operador adjunto. Uma atenção especial será dada aos operadores autoadjuntos, como premissa para a construção do fluxo espectral. Estes resultados serão de grande importância no resto do trabalho.

Na terceira seção mostraremos algumas noções básicas da teoria espectral. Lembraremos a definição do espectro de um operador limitado. Além disso, para um operador auto-adjunto $L \in L(H)$, onde $H$ é um espaço de Hilbert, veremos a decomposição espectral de $H$ na soma direta dos subespaços espectrais positivo e negativo e o núcleo do operador $L$.

Na quarta seção provaremos que, se $H$ é um espaço de Hilbert de dimensão infinita, real e separável, o conjunto dos operadores de Fredholm auto-adjuntos, que denotaremos por $\Phi_{S}(H)$, possui três componentes conexas ligadas à estrutura dos subespaços espectrais.

Nas três primeiras seções, se não se diz o contrario, $H$ representará um espaço de Hilbert de dimensão finita ou infinita, com produto interno denotado por $\langle\cdot, \cdot\rangle$, sobre o corpo $\mathbb{K}$, onde $\mathbb{K}$ é $\mathbb{R}$ ou $\mathbb{C}$, em quanto a quarta seção $H$ será considerado real. 


\subsection{Preliminares: algumas propriedades dos espa- ços de Hilbert}

Como tínhamos falado acima, sublinharemos alguns fatos particulares inerentes aos espaços de Hilbert (reais ou complexos) e aos operadores limitados em espaços de Hilbert que serão usados no resto do trabalho. O leitor pode observar que alguns dos resultados são válidos no caso em que $H$ seja um espaço com produto interno não necessariamente completo.

Uma das propriedades do produto interno é a continuidade, como mostraremos no seguinte lema.

Lema 3.1.1 (Continuidade do produto interno). Se $\left(x_{n}\right)_{n=1}^{\infty} e\left(y_{n}\right)_{n=1}^{\infty}$ são duas sequências em $H$ tais que $x_{n} \rightarrow x$ e $y_{n} \rightarrow y$, onde $x, y \in H$, então $\left\langle x_{n}, y_{n}\right\rangle \rightarrow\langle x, y\rangle$.

Demonstração. Da definição do produto interno e da desigualdade de Cauchy-Schwarz (ver (1.1.2)), se segue

$\left|\langle x, y\rangle-\left\langle x_{n}, y_{n}\right\rangle\right|=\left|\langle x, y\rangle-\left\langle x_{n}, y\right\rangle+\left\langle x_{n}, y\right\rangle-\left\langle x_{n}, y_{n}\right\rangle\right| \leq\left\|x-x_{n}\right\|\|y\|+\left\|x_{n}\right\|\left\|y-y_{n}\right\|$.

Como $\left\|x-x_{n}\right\| \rightarrow 0$ e $\left\|y-y_{n}\right\| \rightarrow 0$, temos $\left\langle x_{n}, y_{n}\right\rangle \rightarrow\langle x, y\rangle$.

Definição 3.1.2. Seja $A$ um subconjunto de $H$. Dizemos que $x \in H$ é ortogonal a $A$, se $\langle x, y\rangle=0$ para todo $y \in A$. O conjunto ortogonal de um subconjunto $A$ de $H$, denotado por $A^{\perp}$, é o conjunto dos $x \in H$ que são ortogonais a $A$. Se $A$ é um subespaço de $H, A^{\perp}$ será chamado de complementar ortogonal de $A$.

Vejamos algumas das propriedades que possui um conjunto ortogonal.

Proposição 3.1.3. Se $A$ é um subconjunto de $H$, então $A^{\perp}$ é um subespaço fechado de $H$ e $A^{\perp}=\bar{A}^{\perp}$.

Demonstração. Primeiro vejamos que $A^{\perp}$ é um subespaço de $H$. De fato, sejam $x, y \in$ $A^{\perp}$ e $\lambda \in \mathbb{K}$. Assim, se $a \in A$, temos

$$
\langle\lambda x+y, a\rangle=\langle\lambda x, a\rangle+\langle y, a\rangle=\lambda\langle x, a\rangle+\langle y, a\rangle=0
$$

isto é, $\lambda x+y \in A^{\perp}$. Este fato prova que $A^{\perp}$ é um subespaço de $H$.

Agora mostremos que $A^{\perp}$ é fechado. Seja $\left(x_{n}\right)_{n=1}^{\infty}$ uma sequência em $A^{\perp}$ convergente a $x \in H$. Daí, se $a \in A$ e $n \in \mathbb{N}$, a desigualdade de Cauchy-Schwarz implica que

$$
|\langle x, a\rangle|=\left|\langle x, a\rangle-\left\langle x_{n}, a\right\rangle\right|=\left|\left\langle x-x_{n}, a\right\rangle\right| \leq\left\|x-x_{n}\right\|\|a\| .
$$

Dado que $x_{n} \rightarrow x$, então $\langle x, a\rangle=0$ para todo $a \in A$, isto é, $x \in A^{\perp}$, como se queria provar. 
Por último, provemos que $A^{\perp}=\bar{A}^{\perp}$. É claro que $\bar{A}^{\perp} \subseteq A^{\perp}$, pois $A \subseteq \bar{A}$. Por outro lado, seja $x \in A^{\perp}$. Qualquer $a \in \bar{A}$ é o limite de uma sequência $\left(a_{n}\right)_{n=1}^{\infty}$ em $A$. Assim,

$$
|\langle x, a\rangle|=\left|\langle x, a\rangle-\left\langle x, a_{n}\right\rangle\right|=\left|\left\langle x, a_{n}-a\right\rangle\right| \leq\|x\|\left\|a_{n}-a\right\| .
$$

Portanto, $\langle x, a\rangle=0$ e $a \in \bar{A}^{\perp}$. Consequentemente, $A^{\perp}=\bar{A}^{\perp}$.

Teorema 3.1.4 (Soma direta). Seja $H_{1}$ um subespaço completo de $H$, isto é, $H_{1}$ é um espaço de Banach. Então,

$$
H=H_{1} \oplus H_{1}^{\perp} .
$$

Demonstração. Seja $x \in H$ fixado. Provemos que existe $x_{1} \in H_{1}$ tal que

$$
\left\|x-x_{1}\right\|=\inf _{z \in H_{1}}\|x-z\|
$$

e $x-x_{1} \perp H_{1}$. De fato, para $n \in \mathbb{N}$, seja $y_{n} \in H_{1}$ tal que $\left\|x-y_{n}\right\|^{2}<d^{2}+1 / n$, onde $d=\inf _{z \in H_{1}}\|x-z\|$. Vejamos que $\left(y_{n}\right)_{n=1}^{\infty}$ é uma sequência de Cauchy. Consideremos $x-y_{n}$ e $x-y_{m}$. Da lei do paralelogramo temos

$$
\left\|\left(x-y_{n}\right)+\left(x-y_{m}\right)\right\|^{2}+\left\|\left(x-y_{n}\right)-\left(x-y_{m}\right)\right\|^{2}=2\left\|x-y_{n}\right\|^{2}+2\left\|x-y_{m}\right\|^{2} .
$$

Assim, como $\frac{y_{n}-y_{m}}{2} \in H_{1}$, então

$$
\begin{aligned}
\left\|y_{m}-y_{n}\right\|^{2} & =2\left\|x-y_{n}\right\|^{2}+2\left\|x-y_{m}\right\|^{2}-\left\|2 x-\left(y_{n}-y_{m}\right)\right\|^{2} \\
& =2\left\|x-y_{n}\right\|^{2}+2\left\|x-y_{m}\right\|^{2}-4\left\|x-\frac{y_{n}-y_{m}}{2}\right\|^{2} \\
& \leq 2\left(d^{2}+1 / n\right)+2\left(d^{2}+1 / m\right)-4 d^{2}=\frac{2}{n}+\frac{2}{m} .
\end{aligned}
$$

Consequentemente, dado que $H_{1}$ é completo, $\left(y_{n}\right)_{n=1}^{\infty}$ converge a algum ponto $x_{1} \in H_{1}$. Portanto,

$$
\left\|x-x_{1}\right\|=\lim _{n \rightarrow \infty}\left\|x-y_{n}\right\|=d .
$$

Ponhamos $x_{2}=x-x_{1}$. Assumamos que $x_{2}$ não é ortogonal a $H_{1}$. Assim, existe $z \in H_{1}$ tal que $\left\langle x_{2}, z\right\rangle>0$. Então, para $\varepsilon>0$, temos

$$
\begin{aligned}
\left\|x-\left(x_{1}+\varepsilon z\right)\right\|^{2} & =\left\|x_{2}-\varepsilon z\right\|^{2}=\left\langle x_{2}-\varepsilon z, x_{2}-\varepsilon z\right\rangle \\
& =\left\langle x_{2}, x_{2}\right\rangle-2 \varepsilon\left\langle x_{2}, z\right\rangle+\varepsilon^{2}\langle z, z\rangle \\
& =d^{2}-\varepsilon\left(2\left\langle x_{2}, z\right\rangle-\varepsilon\|z\|^{2}\right) .
\end{aligned}
$$

Já que $\left\langle x_{2}, z\right\rangle>0$, para $\varepsilon$ suficientemente pequeno, $2\left\langle x_{2}, z\right\rangle-\varepsilon\|z\|^{2}>0$. Daí, $\| x-$ $\left(x_{1}+\varepsilon z\right) \|<d$, contradizendo a definição de $d$. Assim, $x_{2} \perp H_{1}$. Logo, para qualquer $x \in H$ podemos achar $x_{1} \in H_{1}, x_{2} \in H_{1}^{\perp}$ tal que $x=x_{1}+x_{2}$. Em conclusão,

$$
H=H_{1}+H_{1}^{\perp} .
$$

É claro que $H_{1} \cap H_{1}^{\perp}=\{0\}$. Consequentemente, $H=H_{1} \oplus H_{1}^{\perp}$. 
Observe que o teorema anterior é válido também no caso em que $H$ seja um espaço com produto interno não necessariamente completo. Como consequência obtemos que, para qualquer subespaço completo $H_{1}$ de um espaço com produto interno $H$, existe um complementar fechado $H_{2}=H_{1}^{\perp}$ de $H_{1}$, isto é, $H=H_{1} \oplus H_{2}$. Por outro lado, se $E$ é um espaço normado (ou de Banach), que não tem estrutura de espaço com produto interno, este fato não é sempre válido, isto é, existem subespaços completos de $E$ que não têm complementar fechado. Porém, como vimos na Proposição 1.2.4, todo subespaço de dimensão finita de um espaço normado possui um complementar fechado.

Outra propriedade que possui o complementar ortogonal e que será usada nas próximas seções é dada na seguinte proposição.

Proposição 3.1.5. Se $H_{1}$ é um subespaço de $H$, então $\bar{H}_{1}=H_{1}^{\perp \perp}$.

Demonstração. Seja $x \in H_{1}$ fixado. Então, $\langle x, y\rangle=0$ para todo $y \in H_{1}^{\perp}$. Assim, $x \in H_{1}^{\perp \perp}$. Este fato prova que $H_{1} \subseteq H_{1}^{\perp \perp}$. Daí, $\bar{H}_{1} \subseteq H_{1}^{\perp \perp}$, pois $H_{1}^{\perp \perp}$ é fechado.

Por outro lado, se segue do teorema anterior que $H=\bar{H}_{1} \oplus \bar{H}_{1}{ }^{\perp}$. Logo, se $x \in H_{1}^{\perp \perp}$, então $x=y+z$ onde $y \in \bar{H}_{1} \subseteq H_{1}^{\perp \perp} \mathrm{e} z \in \bar{H}_{1}{ }^{\perp}$. Dado que $H_{1}^{\perp \perp}$ é um espaço vetorial, temos $z=x-y \in H_{1}{ }^{\perp \perp}$. Portanto, $\langle v, z\rangle=0$ para todo $v \in H_{1}^{\perp}=\bar{H}_{1}{ }^{\perp}$. Em particular, $\langle z, z\rangle=0$, pois $z \in H_{1}^{\perp}$. Isto é, $z=0$, e $x=y \in H_{1}$. Consequentemente, $\bar{H}_{1}=H_{1}^{\perp \perp}$.

Observe que, se $H=H_{1} \oplus H_{2}$, onde $H_{1}$ e $H_{2}$ são dois subespaços ortogonais de $H$, então, para $x_{1}, y_{1} \in H_{1}$ e $x_{2}, y_{2} \in H_{2}$, temos

$$
\left\langle x_{1}+x_{2}, y_{1}+y_{2}\right\rangle=\left\langle x_{1}, y_{1}\right\rangle+\left\langle x_{2}, y_{2}\right\rangle .
$$

Portanto, se $x=x_{1}+x_{2}$, então

$$
\|x\|=\sqrt{\left\|x_{1}\right\|^{2}+\left\|x_{2}\right\|^{2}} .
$$

A igualdade acima é conhecida como o Teorema de Pitágoras: Se $x_{1}$ e $x_{2}$ são dois elementos ortogonais de um espaço com produto interno, então

$$
\left\|x_{1}+x_{2}\right\|=\sqrt{\left\|x_{1}\right\|^{2}+\left\|x_{2}\right\|^{2}} .
$$

Por outro lado, se $H_{1}$ e $H_{2}$ são espaços de Hilbert com produtos internos denotados por $\langle\cdot, \cdot\rangle_{1}$ e $\langle\cdot, \cdot\rangle_{2}$, respectivamente, então $H=H_{1} \times H_{2}$ é um espaço de Hilbert com o produto interno dado por

$$
\left\langle\left(x_{1}, x_{2}\right),\left(y_{1}, y_{2}\right)\right\rangle=\left\langle x_{1}, y_{1}\right\rangle_{1}+\left\langle x_{2}, y_{2}\right\rangle_{2} \quad \text { para } x_{1}, y_{1} \in H_{1} \text { e } x_{2}, y_{2} \in H_{2} .
$$


Assim,

$$
\left\|\left(x_{1}, x_{2}\right)\right\|=\sqrt{\left\|x_{1}\right\|_{1}^{2}+\left\|x_{2}\right\|_{2}^{2}} \quad \text { para }\left(x_{1}, x_{2}\right) \in H_{1} \times H_{2} .
$$

Note que com este produto interno $H_{1} \times\{0\}$ e $\{0\} \times H_{2}$ são subespaços ortogonais de $H=H_{1} \times H_{2}$ e

$$
H=\left[H_{1} \times\{0\}\right] \oplus\left[\{0\} \times H_{2}\right] .
$$

\subsection{Operadores em espaços de Hilbert}

O fluxo espectral será definido para caminhos de operadores de Fredholm auto-adjuntos. Nesta seção lembraremos o conceito de operador adjunto para operadores em espaços com produto interno. Além disso, veremos algumas propriedades que ele possui e que servirão de ferramenta útil nas próximas seções.

Definição 3.2.1 (Operador adjunto em espaços de Hilbert). Seja $T: H_{1} \rightarrow H_{2}$ um operador limitado, onde $H_{1}$ e $H_{2}$ são espaços de Hilbert. Então, o operador adjunto $T^{*}$ de $T$ é o operador $T^{*}: H_{2} \rightarrow H_{1}$ tal que, para cada $x \in H_{1}$ e $y \in H_{2}$,

$$
\langle T x, y\rangle=\left\langle x, T^{*} y\right\rangle .
$$

O seguinte teorema mostra que esta definição faz sentido e que, além disso, $\|T\|=$ $\left\|T^{*}\right\|$. Podemos ver uma prova deste fato em [16], pág. 196, Teorema 3.9-2.

Teorema 3.2.2. $O$ operador adjunto $T^{*}$ de $T$ na definição anterior existe, é único e é um operador linear limitado com norma

$$
\|T\|=\left\|T^{*}\right\| .
$$

Apresentaremos no seguinte teorema algumas propriedades do operador adjunto que vão se usar neste trabalho. Uma prova se pode ver, por exemplo, em [16], pág. 198, Teorema 3.9-4.

Teorema 3.2.3. Sejam $H_{1}, H_{2}$ espaços de Hilbert, $L: H_{1} \rightarrow H_{2}$ e $T: H_{1} \rightarrow H_{2}$ operadores limitados e $\lambda \in \mathbb{K}$. Então,

1) $(L+T)^{*}=L^{*}+T^{*}$.

2) $(\lambda L)^{*}=\bar{\lambda} L^{*}$.

3) $\left(L^{*}\right)^{*}=L$.

4) $\left\|L^{*} L\right\|=\left\|L L^{*}\right\|=\|L\|^{2}$.

5) $(L T)^{*}=T^{*} L^{*}$, assumindo $H_{1}=H_{2}$. 
Vejamos uma propriedade que satisfaz o adjunto do produto direto de dois operadores limitados definidos em espaços de Hilbert. De fato, suponhamos que $H_{1}$ e $H_{2}$ sejam dois espaços de Hilbert reais ou complexos $\left(H_{1} \times H_{2}\right.$ é um espaço de Hilbert com o produto interno definido em (3.1.1)) e que $L_{1} \in L\left(H_{1}\right)$ e $L_{2} \in L\left(H_{2}\right)$. Mostremos que

$$
\left(L_{1}, L_{2}\right)^{*}=\left(L_{1}^{*}, L_{2}^{*}\right) .
$$

Se $\left(x_{1}, x_{2}\right) \in H_{1} \times H_{2}$ e $\left(y_{1}, y_{2}\right) \in H_{1} \times H_{2}$, se segue de (3.1.1) que

$$
\begin{aligned}
\left\langle\left(L_{1}, L_{2}\right)\left(x_{1}, x_{2}\right),\left(y_{1}, y_{2}\right)\right\rangle & =\left\langle\left(L_{1} x_{1}, L_{2} x_{2}\right),\left(y_{1}, y_{2}\right)\right\rangle \\
& =\left\langle L_{1} x_{1}, y_{1}\right\rangle_{1}+\left\langle L_{2} x_{2}, y_{2}\right\rangle_{2} \\
& =\left\langle x_{1}, L_{1}^{*} y_{1}\right\rangle_{1}+\left\langle x_{2}, L_{2}^{*} y_{2}\right\rangle_{2} \\
& =\left\langle\left(x_{1}, x_{2}\right),\left(L_{1}^{*} y_{1}, L_{2}^{*} y_{2}\right)\right\rangle \\
& =\left\langle\left(x_{1}, x_{2}\right),\left(L_{1}^{*}, L_{2}^{*}\right)\left(y_{1}, y_{2}\right)\right\rangle .
\end{aligned}
$$

A unicidade do operador adjunto implica que

$$
\left(L_{1}, L_{2}\right)^{*}=\left(L_{1}^{*}, L_{2}^{*}\right) .
$$

Por outro lado, se $H=H_{1} \oplus H_{2}$, onde $H_{1}$ e $H_{2}$ são dois subespaços fechados e ortogonais de $H$, invariantes por um operador $L \in L(H), L=L_{1} \oplus L_{2}$, onde $L_{1}$ e $L_{2}$ são as restrições de $L$ a $H_{1}$ e $H_{2}$, respectivamente (veja-se (1.2.1)). De forma análoga ao fato anterior podemos provar que

$$
L^{*}=\left(L_{1} \oplus L_{2}\right)^{*}=L_{1}^{*} \oplus L_{2}^{*} .
$$

Definição 3.2.4. Suponhamos que $H_{1} H_{2}$ sejam espaços de Hilbert. Diremos que $L \in L\left(H_{1}, H_{2}\right)$ é ortogonal se $L^{*} L=I_{H_{1}}$ e $L L^{*}=I_{H_{2}}$, onde $I_{H_{1}}$ e $I_{H_{2}}$ são as identidades de $H_{1}$ e $H_{2}$, respectivamente.

Se $H=H_{1}=H_{2}, L \in L(H)$ e $L=L^{*}$, diremos que $L$ é auto-adjunto.

Observação 3.2.5. As propriedades 1 e 2 do Teorema 3.2 .3 implicam que, se $H$ é um espaço de Hilbert real, então o conjunto dos operadores auto-adjuntos em $L(H)$, que denotaremos por $L_{S}(H)$, é um subespaço de $L(H)$. Provemos que $L_{S}(H)$ é fechado. De fato, seja $\left(L_{n}\right)_{n=1}^{\infty}$ uma sequência de operadores auto-adjuntos convergente a $L \in L(H)$. Daí,

$$
\left\|L^{*}-L_{n}\right\|=\left\|L^{*}-L_{n}^{*}\right\|=\left\|\left(L-L_{n}\right)^{*}\right\|=\left\|L-L_{n}\right\| .
$$

Portanto, $\left(L_{n}\right)_{n=1}^{\infty}$ converge a $L^{*} \in L(H)$. Assim, $L=L^{*}$ e $L \in L_{S}(H)$.

No caso complexo podemos ter $T \neq 0$ auto-adjunto, porém, da segunda propriedade do Teorema 3.2.3, iT não é auto-adjunto.

Na seguinte proposição mostraremos uma condição suficiente e necessária para que um operador seja ortogonal. 
Proposição 3.2.6. Um operador $L \in L\left(H_{1}, H_{2}\right)$ é ortogonal se, e somente se, é sobrejetor e

$$
\langle L x, L y\rangle=\langle x, y\rangle \quad \text { para todo } x, y \in H_{1} .
$$

Demonstração. De fato, se $L$ é ortogonal, então $L^{*} L=I_{H_{1}}$ e $L L^{*}=I_{H_{2}}$. Este fato prova que $L$ é inversível e que verifica (3.2.3).

Reciprocamente, suponhamos que $L$ seja sobrejetor e que verifique (3.2.3). A igualdade (3.2.3) e a unicidade do operador adjunto implicam que $L$ é injetor e que $L^{*} L=I$. Como $L$ é sobrejetor, então é um isomorfismo. Daí, $L^{-1}=L^{*}$, como queríamos provar.

A identidade é um clássico exemplo de operador auto-adjunto e ortogonal. No seguinte exemplo apresentaremos um outro operador que é auto-adjunto e ortogonal.

Exemplo 3.2.7. $\mathrm{O}$ operador $L: \ell_{2} \rightarrow \ell_{2}$ definido por

$$
L\left(x_{1}, x_{2}, x_{3}, x_{4}, \ldots\right)=\left(-x_{1}, x_{2},-x_{3}, x_{4}, \ldots\right), \quad \text { onde }\left(x_{1}, x_{2}, x_{3}, x_{4}, \ldots\right) \in \ell_{2},
$$

é auto-adjunto e ortogonal. De fato, sejam $\left(x_{1}, x_{2}, x_{3}, x_{4}, \ldots\right)$ e $\left(y_{1}, y_{2}, y_{3}, y_{4}, \ldots\right)$ em $\ell_{2}$. Assim,

$$
\begin{aligned}
\left\langle L\left(x_{1}, x_{2}, x_{3}, x_{4}, \ldots\right),\left(y_{1}, y_{2}, y_{3}, y_{4}, \ldots\right)\right\rangle & =\left\langle\left(-x_{1}, x_{2},-x_{3}, x_{4}, \ldots\right),\left(y_{1}, y_{2}, y_{3}, y_{4}, \ldots\right)\right\rangle \\
& =\sum_{n=1}^{\infty}(-1)^{n} x_{n} y_{n} \\
& =\left\langle\left(x_{1}, x_{2}, x_{3}, x_{4}, \ldots\right),\left(-y_{1}, y_{2},-y_{3}, y_{4}, \ldots\right)\right\rangle \\
& =\left\langle\left(x_{1}, x_{2}, x_{3}, x_{4}, \ldots\right), L\left(y_{1}, y_{2}, y_{3}, y_{4}, \ldots\right)\right\rangle .
\end{aligned}
$$

Consequentemente, $L=L^{*}$ e assim, $L$ é auto-adjunto. É fácil ver que $L L=I$. Portanto, $L^{-1}=L=L^{*}$, isto é, $L$ é ortogonal.

O Corolário 1.1.5 prova que, se $L \in L\left(H_{1}, H_{2}\right)$, onde $H_{1}$ e $H_{2}$ dois subespaços de um espaço de Hilbert $H$, então existe um único operador $\bar{L}$ em $L\left(\bar{H}_{1}, \bar{H}_{2}\right)$ tal que $\bar{L} x=L x$ para todo $x \in H_{1}$ e $\|\bar{L}\|=\|L\|$. Lembremos que o operador $\bar{L}$ é definido como

$$
\bar{L} \bar{x}=\lim _{n \rightarrow \infty} L x_{n},
$$

onde $\left(x_{n}\right)_{n=1}^{\infty}$ é uma sequência em $H_{1}$ convergente a $\bar{x} \in \bar{H}_{1}$. A seguinte proprosição mostra algumas propriedades que possui o operador $\bar{L}$ que serão usadas para achar a decomposição polar de um operador limitado.

Proposição 3.2.8. Sejam $L \in L\left(H_{1}, H_{2}\right)$ e $\bar{L}$ como acima. Se $L$ é ortogonal (autoadjunto), então $\bar{L}$ é ortogonal (auto-adjunto). 
Demonstração. Provemos que, se $L$ é ortogonal, então $\bar{L}$ é ortogonal. Sejam $\bar{x}, \bar{y}$ em $\bar{H}_{1}$ e $\left(x_{n}\right)_{n=1}^{\infty},\left(y_{n}\right)_{n=1}^{\infty}$ duas sequências em $H_{1}$ convergentes a $\bar{x}, \bar{y}$, respectivamente. Pela definição de $\bar{L}$, temos que $L L^{*} x_{n} \rightarrow \bar{L} \bar{L}^{*} \bar{x}$. Daí, a continuidade do produto interno (Lema 3.1.1) implica que

$$
\left\langle L L^{*} x_{n}, y_{n}\right\rangle \rightarrow\left\langle\bar{L} \bar{L}^{*} \bar{x}, \bar{x}\right\rangle \quad \text { e } \quad\left\langle x_{n}, y_{n}\right\rangle \rightarrow\langle\bar{x}, \bar{y}\rangle .
$$

Dado que $L L^{*} x_{n}=x_{n}$ para todo $n$, então

$$
\left\langle\bar{L} \bar{L}^{*} \bar{x}, \bar{y}\right\rangle=\langle\bar{x}, \bar{y}\rangle
$$

Logo, $\bar{L} \bar{L}^{*}=I$. Analogamente, $\bar{L}^{*} \bar{L}=I$. Em conclusão, o operador $\bar{L}$ é ortogonal.

A prova de que, se $L$ é auto-adjunto, então $\bar{L}$ é auto-adjunto é análoga e portanto é omitida.

Uma propriedade muito conhecida da análise funcional e que também será de grande importância neste trabalho é dada na seguinte proposição.

Proposição 3.2.9. Se $L \in L(H)$, então $\operatorname{Ker} L=\left(\operatorname{Im} L^{*}\right)^{\perp}$.

Demonstração. Seja $x \in \operatorname{Ker} L$ fixado. Se $y \in \operatorname{Im} L^{*}$, então $y=L^{*} z$ para algum $z \in H$. Assim,

$$
\langle x, y\rangle=\left\langle x, L^{*} z\right\rangle=\langle L x, z\rangle=0 .
$$

Logo, $x \in\left(\operatorname{Im} L^{*}\right)^{\perp}$ e portanto

$$
\operatorname{Ker} L \subseteq\left(\operatorname{Im} L^{*}\right)^{\perp} .
$$

Agora, suponhamos que $x \in\left(\operatorname{Im} L^{*}\right)^{\perp}$. Então, $\langle x, y\rangle=0$ para todo $y \in \operatorname{Im} L^{*}$. Daí, $\left\langle x, L^{*} z\right\rangle=0$ para todo $z \in H$. Consequentemente, $\langle L x, z\rangle=0$ para todo $z \in H$. Este fato prova que $L x=0$, isto é, $x \in \operatorname{Ker} L$. Portanto,

$$
\left(\operatorname{Im} L^{*}\right)^{\perp} \subseteq \operatorname{Ker} L .
$$

Os dois fatos acima mostram que $\operatorname{Ker} L=\left(\operatorname{Im} L^{*}\right)^{\perp}$.

Das Proposições 3.1.5 e 3.2.9 segue-se $(\operatorname{Ker} L)^{\perp}=\overline{\operatorname{Im} L^{*}}$. Assim,

$$
H=\overline{\operatorname{Im} L^{*}} \oplus \operatorname{Ker} L .
$$

O seguinte corolário é uma consequência imediata da proposiçao anterior.

Corolário 3.2.10. Se L é um operador auto-adjunto, então L é o operador zero no complementar ortogonal de $\operatorname{Im} L$. 
Demonstração. Da proposição anterior temos

$$
(\operatorname{Im} L)^{\perp}=\left(\operatorname{Im} L^{*}\right)^{\perp}=\operatorname{Ker} L .
$$

Consequentemente, $L$ é o operador zero no complementar ortogonal de $\operatorname{Im} L$.

Definição 3.2.11. Uma projeção ortogonal é um operador $P \in L(H)$ tal que $P^{2} x=$ $P x$ para todo $x \in H$ e $\operatorname{Im} P=(\operatorname{Ker} P)^{\perp}$.

Como veremos no pŕoximo exemplo, existem operadores $P \in L(H)$ tais que $P^{2}=P$ que não são projeções ortogonais.

Exemplo 3.2.12. Seja $P: \mathbb{R}^{2} \rightarrow \mathbb{R}^{2}$ definido por $P(x, y)=(x, x)$ para $(x, y) \in \mathbb{R}^{2}$. É fácil ver que $P^{2}=P$. Além disso,

$$
\operatorname{Im} P=\{(x, x): x \in \mathbb{R}\} \quad \text { e } \quad \operatorname{Ker} P=\{(0, y): y \in \mathbb{R}\} .
$$

Portanto, $\operatorname{Im} P$ e Ker $L$ não são ortogonais, isto é, $P$ não é uma projeção ortogonal.

Observe que, se $P \in L(H)$ é um operador tal que $P^{2}=P$ e $x \in \operatorname{Im} P$, então $P x=x$. De fato, se $x \in \operatorname{Im} P$, então $x=P z$ para algum $z \in H$. Logo,

$$
P x=P^{2} z=P z=x .
$$

A seguinte é uma caracterização das projeções ortogonais.

Proposição 3.2.13. Seja $P \in L(H)$ fixado. Então, $P$ é uma projeção ortogonal se, $e$ somente se, é auto-adjunto e $P^{2}=P$.

Demonstração. Suponhamos que $P$ seja uma projeção ortogonal. Por definição temos $P^{2}=P$. Também, $\operatorname{Im} P=(\operatorname{Ker} P)^{\perp}$. Este fato prova que $\operatorname{Im} P$ é fechado, pois $(\operatorname{Ker} P)^{\perp}$ é fechado. Como $P$ é contínua, então Ker $P$ é fechado. Daí, o Teorema 3.1.4 implica que

$$
H=\operatorname{Im} P \oplus \operatorname{Ker} P .
$$

Assim, se $x, y \in H$, então $x=x_{1}+x_{2}$ e $y=y_{1}+y_{2}$ onde $x_{1}, y_{1} \in \operatorname{Im} P$ e $x_{2}, y_{2} \in$ Ker $P$. Portanto, $P x_{1}=x_{1}, P y_{1}=y_{1} \mathrm{e}$

$$
\langle P x, y\rangle=\left\langle P\left(x_{1}+x_{2}\right), y_{1}+y_{2}\right\rangle=\left\langle P x_{1}, y_{1}\right\rangle+\left\langle P x_{1}, y_{2}\right\rangle=\left\langle P x_{1}, P y_{1}\right\rangle,
$$

pois $P x_{1} \in \operatorname{Im} P$ e $y_{2} \in \operatorname{Ker} P$. Por outro lado,

$$
\langle x, P y\rangle=\left\langle x_{1}+x_{2}, P\left(y_{1}+y_{2}\right)\right\rangle=\left\langle x_{1}, P y_{1}\right\rangle+\left\langle x_{2}, P y_{2}\right\rangle=\left\langle P x_{1}, P y_{1}\right\rangle .
$$

Consequentemente, $\langle P x, y\rangle=\langle x, P y\rangle$ para todo $x, y \in H$ e $P$ é auto-adjunto. 
Reciprocamente, suponhamos que $P$ seja auto-adjunto e que $P^{2}=P$. Como $P$ é auto-adjunto, de (3.2.5) temos

$$
H=\overline{\operatorname{Im} P} \oplus \operatorname{Ker} P,
$$

onde $\overline{\operatorname{Im} P}$ e $(\operatorname{Ker} P)^{\perp}$ são ortogonais. Portanto, $\operatorname{Im} P$ e $(\operatorname{Ker} P)^{\perp}$ são ortogonais. Por outro lado, se $x \in H$, então $x=P x+x-P x$. Dado que $P x \in \operatorname{Im} P$ e $x-P x \in \operatorname{Ker} P$, se segue

$$
H=\operatorname{Im} P \oplus \operatorname{Ker} P .
$$

Assim, dado que $\operatorname{Im} P \subseteq \overline{\operatorname{Im} P}$, de (3.2.6) temos $\overline{\operatorname{Im} P}=\operatorname{Im} P$ e $\operatorname{Im} P=(\operatorname{Ker} P)^{\perp}$. Em conclusão, $P$ é uma projeção ortogonal.

O seguinte corolário é uma consequência da proposição anterior.

Corolário 3.2.14. Se $P$ e $Q$ são projeções ortogonais que comutam, então $P Q$ é a projeção ortogonal sobre $\operatorname{Im} P \cap \operatorname{Im} Q$.

Demonstração. Dado que

$$
(P Q)^{2}=P Q P Q=P^{2} Q^{2}=P Q \quad \text { e }(P Q)^{*}=Q^{*} P^{*}=Q P=P Q,
$$

então, pela Proposição 3.2.13, $P Q$ é uma projeção ortogonal.

Se $x \in \operatorname{Im} P \cap \operatorname{Im} Q$, temos $P x=x=Q x$. Daí, $P Q x=P x=x$, isto é, $P Q$ é a identidade em $\operatorname{Im} P \cap \operatorname{Im} Q$. Portanto,

$$
\operatorname{Im} P \cap \operatorname{Im} Q \subseteq \operatorname{Im} P Q .
$$

Por outro lado, suponhamos que $x \in H$. Assim, $P Q x \in \operatorname{Im} P$ e $Q P x \in \operatorname{Im} Q$. Logo, $P Q x=Q P x \in \operatorname{Im} P \cap \operatorname{Im} Q$. Então,

$$
\operatorname{Im} P Q \subseteq \operatorname{Im} P \cap \operatorname{Im} Q .
$$

Consequentemente, $\operatorname{Im} P Q=\operatorname{Im} P \cap \operatorname{Im} Q$. Os fatos acima provam que $P Q$ é a projeção sobre $\operatorname{Im} P \cap \operatorname{Im} Q$.

Finalizaremos esta seção com uma propriedade importante dos operadores autoadjuntos.

Teorema 3.2.15. Se $L \in L(H)$ é um operador auto-adjunto, então

$$
\|L\|=\sup _{\|x\|=1}|\langle L x, x\rangle| .
$$


Demonstração. Da desigualdade de Cauchy-Schwarz temos

$$
\sup _{\|x\|=1}|\langle L x, x\rangle| \leq \sup _{\|x\|=1}\|L x\|\|x\|=\|L\| .
$$

Provemos agora que $\|L\| \leq \sup _{\|x\|=1}|\langle L x, x\rangle|$. De fato, se $L z=0$ para todo $z \in H$ com $\|z\|=1$, então $L=0$. Assim,

$$
0=\|L\|=\sup _{\|x\|=1}|\langle L x, x\rangle| .
$$

No caso contrario, para qualquer $z \in H$ de norma 1 tal que $L z \neq 0$, tomemos

$$
v=\|L z\|^{1 / 2} z \quad \text { e } \quad w=\|L z\|^{-1 / 2} L z .
$$

Então,

$$
\|v\|^{2}=\|L z\|\|z\|^{2}=\|L z\|=\|L z\|^{-1}\|L z\|^{2}=\|w\|^{2} .
$$

Tomemos agora $y_{1}=v+w$ e $y_{2}=v-w$. Logo,

$$
\begin{aligned}
& \left\langle L y_{1}, y_{1}\right\rangle-\left\langle L y_{2}, y_{2}\right\rangle \\
& \quad=\langle L(v+w), v+w\rangle-\langle L(v-w), v-w\rangle \\
& \quad=\langle L v, v\rangle+\langle L v, w\rangle+\langle L w, v\rangle+\langle L w, w\rangle-\langle L v, v\rangle+\langle L v, w\rangle+\langle L w, v\rangle-\langle L w, w\rangle \\
& \quad=2(\langle L v, w\rangle+\langle L w, v\rangle) \\
& \quad=2\left(\left\langle L\left(\|L z\|^{1 / 2} z\right),\|L z\|^{-1 / 2} L z\right\rangle+\left\langle L\left(\|L z\|^{-1 / 2} L z\right),\|L z\|^{1 / 2} z\right\rangle\right) \\
& \quad=2(\langle L z, L z\rangle+\langle L L z, z\rangle) \\
& =2\left(\langle L z, L z\rangle+\left\langle L z, L^{*} z\right\rangle\right) \\
& =2(\langle L z, L z\rangle+\langle L z, L z\rangle) \\
& =4\|L z\|^{2}
\end{aligned}
$$

isto é,

$$
\left\langle L y_{1}, y_{1}\right\rangle-\left\langle L y_{2}, y_{2}\right\rangle=4\|L z\|^{2} .
$$

Agora, para todo $y \neq 0$ e $u=\|y\|^{-1} y$, temos $y=\|y\| u$ e

$$
|\langle L y, y\rangle|=|\langle L(\|y\| u),\|y\| u\rangle|=\|y\|^{2}|\langle L u, u\rangle| \leq\|y\|^{2} \sup _{\|x\|=1}|\langle L x, x\rangle| .
$$

Portanto, da desigualdade triangular e de (3.2.7) obtemos

$$
\begin{aligned}
\left|\left\langle L y_{1}, y_{1}\right\rangle-\left\langle L y_{2}, y_{2}\right\rangle\right| & \leq\left|\left\langle L y_{1}, y_{1}\right\rangle\right|+\left|\left\langle L y_{2}, y_{2}\right\rangle\right| \\
& \leq \sup _{\|x\|=1}|\langle L x, x\rangle|\left(\left\|y_{1}\right\|^{2}+\left\|y_{2}\right\|^{2}\right) \\
& \leq \sup _{\|x\|=1}|\langle L x, x\rangle|(\langle v+w, v+w\rangle+\langle v-w, v-w\rangle) \\
& =2 \sup _{\|x\|=1}|\langle L x, x\rangle|\left(\|v\|^{2}+\|w\|^{2}\right) \\
& =4 \sup _{\|x\|=1}|\langle L x, x\rangle|\|L z\| .
\end{aligned}
$$


Consequentemente, (3.2.8) implica que

$$
4\|L z\|^{2} \leq 4 \sup _{\|x\|=1}|\langle L x, x\rangle|\|L z\| .
$$

Daí, $\|L z\| \leq \sup _{\|x\|=1}|\langle L x, x\rangle|$. Este fato prova que

$$
\sup _{\|x\|=1}\|L z\| \leq \sup _{\|x\|=1}|\langle L x, x\rangle| .
$$

Em conclusão, $\sup _{\|x\|=1}\|L z\|=\sup _{\|x\|=1}|\langle L x, x\rangle|$.

\subsection{Noções básicas da teoria espectral em espaços normados}

Embora o argumento principal da tese, o fluxo espectral, envolva operadores em espaços de Hilbert, nesta seção vamos apresentar algumas definições e noções básicas da teoria espectral para operadores limitados em espaços normados. Em muitos textos os conceitos básicos da teoria espectral são apresentados para operadores $L: D(L) \subseteq E \rightarrow E$, ou seja, para operadores definidos em subespaços de $E$. Para os nossos fins, esse nível de generalidade não é necessário e portanto somente serão tratados os resultados da teoria espectral no caso particular de operadores definidos em todo o espaço. As propriedades mais interessantes serão dadas para operadores em espaços de Hilbert.

Fazendo uso do conceito de decomposição polar de um operador auto-adjunto $L \in$ $L(H)$, onde $H$ é um espaço de Hilbert, daremos a definição dos subespaços espectrais positivo e negativo do operador $L$, denotados por $H_{+}(L)$ e $H_{-}(L)$, respectivamente. Além disso, mostraremos que

$$
H=H_{+}(L) \oplus H_{-}(L) \oplus \operatorname{Ker} L
$$

e que $H_{+}(L)$ e $H_{-}(L)$ são invariantes por $L$.

Na primeira parte da seção trataremos operadores em espaços normados (não necessariamente com produto interno) e por último com operadores auto-adjuntos em espaços de Hilbert.

Se não se diz o contrario, $E$ denotará um espaço normado sobre $\mathbb{K}$, onde $\mathbb{K}=\mathbb{R}$ (ou $\mathbb{C}$ ).

Definição 3.3.1 (Espectro de um operador). Seja $L$ um operador em $L(E)$. Um valor regular de $L$ é um número $\lambda \in \mathbb{K}$ tal que o operador $L_{\lambda}=L-\lambda I$ é inversível em $L(E)$, isto é, $L_{\lambda}^{-1}$ existe e pertence a $L(E)$. O conjunto de todos os valores regulares de $L$, denotado por $\rho(L)$, é chamado de conjunto resolvente de $L$. Seu complementar $\sigma(L)=\mathbb{K}-\rho(L)$ é chamado de espectro de $L$. Dizemos que $\lambda$ é um valor espectral de 
$L$ se $\lambda \in \sigma(L)$. Para $\lambda \in \rho(L)$, o operador $R(\lambda)=(L-\lambda I)^{-1}$ é chamado de resolvente de $L$.

Um autovalor de um operador $L$ é um valor $\lambda \in \mathbb{K}$ tal que $L-\lambda I$ não é injetor. Se $\lambda$ é um autovalor de $L$, existe um elemento $v \in E \backslash\{0\}$ tal que $L v=\lambda v$. O elemento $v$ é chamado de autovetor de $L$ correspondente ao autovalor $\lambda$. O autoespaço de um autovalor $\lambda$ de $L$ é o subespaço gerado pelos autovetores do operador $L$ correspondentes a $\lambda$.

Teorema 3.3.2. Se $L \in L(E)$, onde $E$ é um espaço de Banach complexo, então $\sigma(L) \neq \emptyset$.

Podemos ver uma prova do teorema anterior em [7], pág. 196, Teorema 3.6.

Quando $E$ tem dimensão finita, o espectro de um operador $L \in L(E)$ é composto pelos autovalores de $L$. Este resultado é consequência do fato que todo operador linear $L$ em um espaço normado de dimensão finita é inversível se, e somente se, é injetor. No seguinte exemplo veremos que em dimensão infinita podemos ter $\lambda \in \sigma(L) \operatorname{com} L-\lambda I$ injetor.

Exemplo 3.3.3. Consideremos o espaço de Hilbert $\ell_{2}$ das sequências de números complexos $\left(x_{n}\right)_{n=1}^{\infty}$ tais que $\sum_{n=1}^{\infty}\left|x_{n}\right|^{2}$ é convergente. O produto interno é dado por

$$
\langle x, y\rangle=\sum_{n=1}^{\infty} x_{n} \bar{y}_{n}, \quad \text { onde } x=\left(x_{n}\right)_{n=1}^{\infty}, y=\left(y_{n}\right)_{n=1}^{\infty} \in \ell_{2} .
$$

Tomemos $L: \ell_{2} \rightarrow \ell_{2}$ definido por

$$
L\left(a_{1}, a_{2}, a_{3}, \ldots\right)=\left(0, a_{1}, a_{2}, a_{3}, \ldots\right), \quad \text { onde }\left(a_{1}, a_{2}, a_{3}, \ldots\right) \in \ell_{2} .
$$

É fácil ver que $L$ é injetor e que $\|L\|=1$. O operador $L$ não é sobrejetor, pois $(1,0,0, \ldots) \notin \operatorname{Im} L$. Assim, $0 \in \sigma(L)$. Provemos que

$$
\sigma(L)=B=\{x \in \mathbb{C}:\|x\| \leq 1\} .
$$

De fato, do Teorema 1.1.7 temos que, se $|\lambda|>1=\|L\|$, então $I-(1 / \lambda) L$ é inversível. Este fato prova que, se $|\lambda|>1$, então o operador $L-\lambda I$ é inversível. Portanto,

$$
\sigma(L) \subseteq B .
$$

Suponhamos que $|\lambda| \leq 1$, com $\lambda \neq 0$. Mostremos que $L-\lambda I$ não é sobrejetor. Tomemos $(1,0,0, \ldots) \in \ell_{2}$. Se existir $\left(a_{1}, a_{2}, a_{3}, \ldots\right) \in \ell_{2}$ tal que $(L-\lambda I)\left(a_{1}, a_{2}, a_{3}, \ldots\right)=$ $(1,0,0, \ldots)$, então

$$
\left(-\lambda a_{1}, a_{1}-\lambda a_{2}, a_{2}-\lambda a_{3} \ldots\right)=(1,0,0, \ldots),
$$

isto é,

$$
a_{1}=-1 / \lambda, \quad a_{2}=-1 / \lambda^{2}, \quad a_{3}=-1 / \lambda^{3}, \ldots .
$$


Dado que $|\lambda| \leq 1$, temos

$$
\left(a_{1}, a_{2}, a_{3}, \ldots\right)=\left(-1 / \lambda,-1 / \lambda^{2},-1 / \lambda^{3}, \ldots\right) \notin \ell_{2} .
$$

Consequentemente, $L-\lambda I$ não é sobrejetor. Daí, $B \subseteq \sigma(L)$. Em conclusão, $B=\sigma(L)$.

No exemplo anterior, observe que, se $\ell_{2}$ fosse considerado sobre o corpo dos números reais (veja-se o Exemplo 2.2.3), então, da Definição 3.3.1 teríamos que $\sigma(L)=[-1,1] \subseteq$ $\mathbb{R}$. Da Análise funcional sabemos que um espaço vetorial real $E$ admite uma 'complexificação', ou seja, um espaço vetorial $\widehat{E}$ sobre $\mathbb{C}$ e uma inclusão linear canônica de $E$ em $\widehat{E}$. Se $E$ for normado (de Banach), ele induz uma estrutura normada (de Banach) em $\widehat{E}$. Assim, um operador linear $L \in L(E)$ admite um operador complexificado $\widehat{L} \in L(\widehat{E})$. Obviamente o espectro de $\widehat{L}$ será contido em $\mathbb{C}$ e podemos chamar $\sigma(\widehat{L})$ como o de espectro de $L$. Portanto, mesmo sendo $L$ um operador entre espaços reais, $\sigma(L)$ será considerado como subconjunto de $\mathbb{C}$. Este fato ajuda profundamente no estudo da propriedades espectrais de $L$.

Por outro lado, a um endomorfismo $L \in L\left(\mathbb{R}^{n}\right)$ é associada uma matriz real (na base canônica do espaço euclidiano) que pode ter autovalores complexos. Consequentemente, em dimensão finita é natural considerar em $\mathbb{C}$ o espectro de um operador real (o de uma matriz real). A complexificação em dimensão infinita estende a possibilidade de definir e usar o espectro em $\mathbb{C}$ de um operador real, com muitas vantagens na investigação. Resumimos este conceito a partir da seguinte definição.

Definição 3.3.4. A complexificação de um espaço real $E$ é o espaço

$$
\widehat{E}=\left\{x_{1}+i x_{2}: x_{1}, x_{2} \in E\right\}
$$

sobre o corpo dos números complexos com as seguintes operações:

i. $\left(x_{1}+i x_{2}\right)+\left(y_{1}+i y_{2}\right)=x_{1}+y_{1}+i\left(x_{2}+y_{2}\right)$ para $x_{1}+i x_{2}, y_{1}+i y_{2} \in \widehat{E}$.

ii. $(a+i b)\left(x_{1}+i x_{2}\right)=a x_{1}-b x_{2}+i\left(b x_{1}+a x_{2}\right)$ para $x_{1}+i x_{2} \in \widehat{E}$ e $a+i b \in \mathbb{C}$. $\widehat{E}$

Mostremos que, se $E$ é normado, com norma denotada por $\|\cdot\|$, então, para $x_{1}+i x_{2} \in$

$$
\left\|x_{1}+i x_{2}\right\|_{\widehat{E}}=\max _{\theta}\left(\left\|\cos \theta x_{1}-\operatorname{sen} \theta x_{2}\right\|^{2}+\left\|\operatorname{sen} \theta x_{1}+\cos \theta x_{2}\right\|^{2}\right)^{1 / 2}
$$

define uma norma em $\widehat{E}$. Seja $x+i y \in \widehat{E}$ fixado. É claro que $\|x+i y\|_{\widehat{E}} \geq 0$ e $\|x+i y\|_{\widehat{E}}=0$ se, e somente se, $x+i y=0$. Não é difícil provar que $\|\cdot\|_{\widehat{E}}$ satisfaz a desigualdade triangular. Vejamos que, se $z=|z|(\cos \alpha+i \operatorname{sen} \alpha) \in \mathbb{C}$, então

$$
\|z(x+i y)\|_{\widehat{E}}=|z|\|x+i y\|_{\widehat{E}}
$$


De fato,

$$
\begin{aligned}
\|z(x+i y)\|_{\widehat{E}}= & \|z \mid[\cos \alpha x-\operatorname{sen} \alpha y+i(\operatorname{sen} \alpha x+\cos \alpha y)]\|_{\widehat{E}} \\
= & \max _{\theta}\left(\||z|[\cos \theta(\cos \alpha x-\operatorname{sen} \alpha y)-\operatorname{sen} \theta(\operatorname{sen} \alpha x+\cos \alpha y)]\|^{2}\right. \\
& \left.+\||| z \mid\left[\operatorname{sen} \theta(\cos \alpha x-\operatorname{sen} \alpha y)+\cos \theta(\operatorname{sen} \alpha x+\cos \alpha y) \|^{2}\right]\right)^{1 / 2} \\
= & |z| \max _{\theta}\left(\|(\cos \theta \cos \alpha-\operatorname{sen} \theta \operatorname{sen} \alpha) x-(\cos \theta \operatorname{sen} \alpha+\operatorname{sen} \theta \cos \alpha) y\|^{2}\right. \\
& \left.+\|(\operatorname{sen} \theta \cos \alpha+\cos \theta \operatorname{sen} \alpha) x-(\operatorname{sen} \theta \operatorname{sen} \alpha-\cos \theta \cos \alpha) y\|^{2}\right)^{1 / 2} \\
= & |z| \max _{\theta}\left(\|\cos (\theta+\alpha) x-\operatorname{sen}(\theta+\alpha) y\|^{2}\right. \\
& \left.+\|\operatorname{sen}(\theta+\alpha) x+\cos (\theta+\alpha) y\|^{2}\right)^{1 / 2} \\
= & |z| \max _{\theta}\left(\|\cos \theta x-\operatorname{sen} \theta y\|^{2}+\|\operatorname{sen} \theta x+\cos \theta y\|^{2}\right)^{1 / 2} \\
= & |z|\|x+i y\|_{\widehat{E}},
\end{aligned}
$$

como queríamos provar.

Observe que, se $E$ é um espaço de Banach, então $\left(\widehat{E},\|\cdot\|_{\widehat{E}}\right)$ é um espaço de Banach. De fato, se $\left(x_{n}+i y_{n}\right)_{n=1}^{\infty}$ é uma sequência de Cauchy em $\widehat{E}$, então, para todo $\varepsilon>0$, existe um inteiro positivo $N$ tal que, se $n, m \geq N$,

$\max _{\theta}\left(\left\|\cos \theta\left(x_{n}-x_{m}\right)-\operatorname{sen} \theta\left(y_{n}-y_{m}\right)\right\|^{2}+\left\|\operatorname{sen} \theta\left(x_{n}-x_{m}\right)+\cos \theta\left(y_{n}-y_{m}\right)\right\|^{2}\right)^{1 / 2}<\varepsilon$.

Logo, tomando $\theta=0,\left(\left\|x_{n}-x_{m}\right\|^{2}+\left\|y_{n}-y_{m}\right\|^{2}\right)^{1 / 2}<\varepsilon$. Assim, $\left\|x_{n}-x_{m}\right\|<\varepsilon$ e $\left\|y_{n}-y_{m}\right\|<\varepsilon$. Daí, $\left(x_{n}\right)_{n=1}^{\infty}$ e $\left(y_{n}\right)_{n=1}^{\infty}$ são sequências de Cauchy em $E$. Dado que $E$ é de Banach, $\left(x_{n}\right)_{n=1}^{\infty}$ é convergente a algum $x \in E$ e $\left(y_{n}\right)_{n=1}^{\infty}$ é convergente a algum $y \in E$. Não é difícil provar que $\left(x_{n}+i y_{n}\right)_{n=1}^{\infty}$ converge a $x+i y \in \widehat{E}$. Consequentemente, $\widehat{E}$ é um espaço de Banach.

Suponhamos agora que $E$ seja um espaço com produto interno denotado por $\langle\cdot, \cdot\rangle$. Vejamos que $\left(\widehat{E},\|\cdot\|_{\widehat{E}}\right)$ é um espaço com produto interno. De fato, se $x_{1}+i x_{2} \in \widehat{E}$, temos

$$
\begin{aligned}
\left\|x_{1}+i x_{2}\right\|_{\widehat{E}}= & \max _{\theta}\left(\left\|\cos \theta x_{1}-\operatorname{sen} \theta x_{2}\right\|^{2}+\left\|\operatorname{sen} \theta x_{1}+\cos \theta x_{2}\right\|^{2}\right)^{1 / 2} \\
= & \max _{\theta}\left(\cos ^{2} \theta\left\|x_{1}\right\|^{2}-2 \cos \theta \operatorname{sen} \theta\left\langle x_{1}, x_{2}\right\rangle+\operatorname{sen}^{2} \theta\left\|x_{2}\right\|^{2}\right. \\
& \left.+\operatorname{sen}^{2} \theta\left\|x_{1}\right\|^{2}+2 \cos \theta \operatorname{sen} \theta\left\langle x_{1}, x_{2}\right\rangle+\cos ^{2} \theta\left\|x_{2}\right\|^{2}\right)^{1 / 2} \\
= & \left(\left\|x_{1}\right\|^{2}+\left\|x_{2}\right\|^{2}\right)^{1 / 2} .
\end{aligned}
$$

Consequentemente,

$$
\left\|x_{1}+i x_{2}\right\|_{\widehat{E}}=\left(\left\|x_{1}\right\|^{2}+\left\|x_{2}\right\|^{2}\right)^{1 / 2} \quad \text { para todo } x_{1}+i x_{2} \in \widehat{E} \text {. }
$$


Provemos que a norma $\|\cdot\|_{\widehat{E}}$ possui a propriedade do paralelogramo, isto é,

$$
\|\widehat{x}+\widehat{y}\|_{\widehat{E}}^{2}+\|\widehat{x}-\widehat{y}\|_{\widehat{E}}^{2}=2\left(\|\widehat{x}\|_{\widehat{E}}^{2}+\|\widehat{y}\|_{\widehat{E}}^{2}\right) \quad \text { para todo } \widehat{x}, \widehat{y} \in \widehat{E} .
$$

Sejam $\widehat{x}=x_{1}+i x_{2}$ e $\widehat{y}=y_{1}+i y_{2} \in \widehat{E}$. Dado que

$$
\left\|x_{1}+y_{1}\right\|^{2}+\left\|x_{1}-y_{1}\right\|^{2}=2\left(\left\|x_{1}\right\|^{2}+\left\|y_{1}\right\|^{2}\right) \text { e }\left\|x_{2}+y_{2}\right\|^{2}+\left\|x_{2}-y_{2}\right\|^{2}=2\left(\left\|x_{2}\right\|^{2}+\left\|y_{2}\right\|^{2}\right),
$$

pois $E$ é um espaço com produto interno, se segue que

$$
\begin{aligned}
\|\widehat{x}+\widehat{y}\|_{\widehat{E}}^{2}+\|\widehat{x}-\widehat{y}\|_{\widehat{E}}^{2} & =\left\|x_{1}+y_{1}+i\left(x_{2}+y_{2}\right)\right\|_{\widehat{E}}^{2}+\left\|x_{1}-y_{1}+i\left(x_{2}-y_{2}\right)\right\|_{\widehat{E}}^{2} \\
& =\left\|x_{1}+y_{1}\right\|^{2}+\left\|x_{2}+y_{2}\right\|^{2}+\left\|x_{1}-y_{1}\right\|^{2}+\left\|x_{2}-y_{2}\right\|^{2} \\
& =2\left(\left\|x_{1}\right\|^{2}+\left\|y_{1}\right\|^{2}\right)+2\left(\left\|x_{2}\right\|^{2}+\left\|y_{2}\right\|^{2}\right) \\
& =2\left(\left\|x_{1}+i x_{2}\right\|_{\widehat{E}}^{2}+\left\|y_{1}+i y_{2}\right\|_{\overparen{E}}^{2}\right) \\
& =2\left(\|\widehat{x}\|_{\widehat{E}}^{2}+\|\widehat{y}\|_{\widehat{E}}^{2}\right) .
\end{aligned}
$$

Consequentemente, $\left(\widehat{E},\|\cdot\|_{\widehat{E}}\right)$ é um espaço com produto interno. Observe que este produto interno é dado por

$$
\left\langle x_{1}+i x_{2}, y_{1}+i y_{2}\right\rangle_{\widehat{E}}=\left\langle x_{1}, y_{1}\right\rangle-i\left\langle x_{1}, y_{2}\right\rangle+i\left\langle x_{2}, y_{1}\right\rangle+\left\langle x_{2}, y_{2}\right\rangle,
$$

para $x_{1}+i x_{2}, y_{1}+i y_{2} \in \widehat{E}$. Os fatos mostrados acima implicam que, se $E$ é um espaço de Hilbert, então $\widehat{E}$ é um espaço de Hilbert.

A complexificação de um operador $L \in L(E)$ é o operador em $\widehat{L} \in L(\widehat{E})$ dado por

$$
\widehat{L}(x+i y)=L(x)+i L(y) \text { para } x+i y \in \widehat{E} .
$$

Assim,

$$
\begin{aligned}
\|L x+i L y\|_{\widehat{E}} & =\max _{\theta}\left(\|\cos \theta L x-\operatorname{sen} \theta L y\|^{2}+\|\operatorname{sen} \theta L x+\cos \theta L y\|^{2}\right)^{1 / 2} \\
& =\max _{\theta}\left(\|L(\cos \theta x-\operatorname{sen} \theta y)\|^{2}+\|L(\operatorname{sen} \theta x+\cos \theta y)\|^{2}\right)^{1 / 2} \\
& \leq \max _{\theta}\|L\|\left[\|\cos \theta x-\operatorname{sen} \theta y\|^{2}+\|\operatorname{sen} \theta x+\cos \theta y\|^{2}\right]^{1 / 2} \\
& =\|L\| \max _{\theta}\left[\|\cos \theta x-\operatorname{sen} \theta y\|^{2}+\|\operatorname{sen} \theta x+\cos \theta y\|^{2}\right]^{1 / 2} \\
& =\|L\|\|x+i y\|_{\widehat{E}}^{2} .
\end{aligned}
$$

Portanto,

$$
\|\widehat{L}\|_{\widehat{E}} \leq\|L\| .
$$

Agora, $\|\widehat{L} x\|_{\widehat{E}}=\|L x\|$ para $x \in E$. Este fato implica que

$$
\|\widehat{L}\|_{\widehat{E}}=\|L\| .
$$


Definição 3.3.5. Definimos o espectro de um operador $L \in L(E)$, onde $E$ é um espaço de normado real, como o espectro da complexificação de $L$.

Uma propriedade do espectro de um operador limitado em um espaço de Banach é a compacidade. Este fato é provado na seguinte proposição.

Proposição 3.3.6. Sejam $E$ um espaço de Banach e $L \in L(E)$. $O$ espectro de $L$ é um subconjunto compacto de $\mathbb{C}$ limitado por $\|L\|$.

Demonstração. Provemos que $\rho(L)$ é aberto em $\mathbb{C}$. De fato, suponhamos que $\lambda_{0} \in \rho(L)$. Assim, $L-\lambda_{0} I$ é inversível. Como $G L(E)$ é aberto (veja-se o Corolário 1.1.8), para $\lambda$ suficientemente perto de $\lambda_{0}$, temos que $L-\lambda I$ é inversível em $L(E)$. Este fato prova que $\rho(L)$ é aberto em $\mathbb{C}$. Assim, $\sigma(L)$ é fechado.

Agora vejamos que $\sigma(L)$ é limitado por $\|L\|$. Se $|\lambda|>\|L\|$, então $\|L / \lambda\|<1$. Se segue do Teorema 1.1.7 que $L / \lambda-I$ é inversível em $L(E)$, portanto $L-\lambda I$ é inversível. Consequentemente, $\lambda \in \rho(L)$. Em conclusão, $\sigma(L)$ é limitado por $\|L\|$.

Outra propriedade do espectro é apresentada na seguinte proposição.

Proposição 3.3.7. Sejam $L \in L(E)$ e $E_{1}$ e $E_{2}$ subespaços fechados de $E$, invariantes por $L$ e tais que $E=E_{1} \oplus E_{2}$. Então,

$$
\sigma(L)=\sigma\left(L_{1}\right) \cup \sigma\left(L_{2}\right),
$$

onde $L_{1}$ e $L_{2}$ são as restrições de $L$ aos subespaços $E_{1}$ e $E_{2}$, respectivamente.

Demonstração. Seja $\lambda \in \mathbb{C}$ fixado. Não é difícil ver que $L-\lambda I$ é inversível se, e somente se, as restrições $L_{1}-\left.\lambda I\right|_{E_{1}}$ e $L_{2}-\left.\lambda I\right|_{E_{2}}$ são inversíveis. Daí, $\lambda \in \rho(L)$ se, e somente se, $\lambda \in \rho\left(L_{1}\right)$ e $\lambda \in \rho\left(L_{2}\right)$. Portanto,

$$
\rho(L)=\rho\left(L_{1}\right) \cap \rho\left(L_{2}\right) .
$$

Este fato prova que $\sigma(L)=\sigma\left(L_{1}\right) \cup \sigma\left(L_{2}\right)$.

Nesta parte da seção apresentaremos algumas propriedades do espectro dos operadores auto-adjuntos em espaços de Hilbert que serão muito úteis de aqui para frente. Se não se diz o contrario, suporemos que $H$ seja um espaço de Hilbert sobre $\mathbb{K}$, onde $\mathbb{K}=$ $\mathbb{R}$ ou $\mathbb{C}$. Abusando um pouco da notação, no caso em que $H$ seja real, identificaremos $L$ com sua complexificação.

Teorema 3.3.8. O espectro de um operador auto-adjunto $L \in L(H)$ é um subconjunto dos números reais. 
Demonstração. Seja $\lambda=a+i b \in \mathbb{C}, \operatorname{com} b \neq 0$. Tomemos $x \in H$ fixado. Dado que $L-a I$ é auto-adjunto, temos

$$
\begin{aligned}
\|(L-\lambda I) x\|^{2} & =\langle(L-(a+i b) I) x,(L-(a+i b) I) x\rangle \\
& =\|(L-a I) x\|^{2}-\langle(L-a I) x, i b x\rangle-\langle i b x,(L-a I) x\rangle+\|b x\|^{2} \\
& =\|(L-a I) x\|^{2}+i\langle(L-a I) x, b x\rangle-i\langle b x,(L-a I) x\rangle+\|b x\|^{2} \\
& =\|(L-a I) x\|^{2}+i\left\langle b x,(L-a I)^{*} x\right\rangle-i\langle b x,(L-a I) x\rangle+\|b x\|^{2} \\
& =\|(L-a I) x\|^{2}+i\langle b x,(L-a I) x\rangle-i\langle b x,(L-a I) x\rangle+\|b x\|^{2} \\
& =\|(L-a I) x\|^{2}+\|b x\|^{2} \\
& \geq|b|\|x\|^{2} .
\end{aligned}
$$

Portanto, $L-\lambda I$ é injetor, pois $|b|>0$. A Proposição 1.1.10 implica que a restrição $(L-\lambda I): H \rightarrow \operatorname{Im}(L-\lambda I)$ do operador $L-\lambda I$ é inversível e que $\operatorname{Im}(L-\lambda I)$ é fechada. Igualmente podemos provar que a restrição $(L-\bar{\lambda} I): H \rightarrow \operatorname{Im}(L-\bar{\lambda} I)$ do operador $L-\bar{\lambda} I$ é inversível. Assim, $\operatorname{Ker}(L-\bar{\lambda} I)=\{0\}$, isto é,

$$
(\operatorname{Ker}(L-\bar{\lambda} I))^{\perp}=H .
$$

Provemos que $\operatorname{Im}(L-\lambda I)=H$. De fato, dado que $\operatorname{Im}(L-\lambda I)$ é fechada, as Proposições 3.1.5 e 3.2.9 e o Teorema 3.2.3 implicam que

$$
\operatorname{Im}(L-\lambda I)=\left(\operatorname{Ker}(L-\lambda I)^{*}\right)^{\perp}=\left(\operatorname{Ker}\left(L^{*}-\bar{\lambda} I\right)\right)^{\perp}=(\operatorname{Ker}(L-\bar{\lambda} I))^{\perp}=H .
$$

Consequentemente, $(L-\lambda I): H \rightarrow H$ é um isomorfismo. Logo, $\lambda \in \rho(L)$.

Fixemos um operador auto-adjunto $L \in L(H)$. Tomemos

$$
m=\inf _{\|x\|=1}\langle L x, x\rangle \quad \text { e } \quad M=\sup _{\|x\|=1}\langle L x, x\rangle .
$$

Se segue do Teorema 3.2.15 que

$$
\|L\|=\sup _{\|x\|=1}|\langle L x, x\rangle|=\max \{|m|,|M|\} .
$$

Da Proposição 3.3.6 e o Teorema 3.3.8 temos que, se $L$ é auto-adjunto, então

$$
\sigma(L) \subseteq[-\|L\|,\|L\|] .
$$

É claro que $[m, M] \subseteq[-\|L\|,\|L\|]$. Na próxima proposição mostraremos que

$$
\sigma(L) \subseteq[m, M] .
$$

Observe que, para $x \in H,\langle L x, x\rangle$ é um número real, pois

$$
\overline{\langle L x, x\rangle}=\langle x, L x\rangle=\langle L x, x\rangle .
$$


Proposição 3.3.9. $O$ espectro de um operador auto-adjunto $L$ está contido no intervalo $[m, M]$, onde $m=\inf _{\|x\|=1}\langle L x, x\rangle$ e $M=\sup _{\|x\|=1}\langle L x, x\rangle$.

Demonstração. Provemos que, se $\lambda=m-c$, onde $c>0$, então $\lambda \in \rho(L)$. De fato, para $y \in H$ com $y \neq 0$, tomemos $z=\|y\|^{-1} y$. Assim, $y=\|y\| z$ e $\|z\|=1$. Portanto,

$$
\langle L y, y\rangle=\|y\|^{2}\langle L z, z\rangle \geq\|y\|^{2} \inf _{\|x\|=1}\langle L x, x\rangle=\langle y, y\rangle m .
$$

Logo, da desigualdade de Cauchy-Schwarz se segue

$$
\|(L-\lambda I) y\|\|y\| \geq\langle(L-\lambda I) y, y\rangle=\langle L y, y\rangle-\langle\lambda y, y\rangle \geq(m-\lambda)\langle y, y\rangle=c\|y\|^{2} .
$$

Daí, $\|(L-\lambda I) y\| \geq c\|y\|$. Dado que $c>0$, a Proposição 1.1 .10 prova que a restrição $L-\lambda I: H \rightarrow \operatorname{Im}(L-\lambda I)$ é inversível e que $\operatorname{Im}(L-\lambda I)$ é fechada.

Provemos agora que o operador $L-\lambda I$ é sobrejetor. Suponhamos por contradição que $\operatorname{Im}(L-\lambda I) \neq H$. Como $\operatorname{Im}(L-\lambda I)$ é fechado, existe $x_{0} \neq 0 \in H$ ortogonal a $\operatorname{Im}(L-\lambda I)$. Já que $L$ é auto-adjunto e $\lambda \in \mathbb{R}$,

$$
0=\left\langle(L-\lambda I) x, x_{0}\right\rangle=\left\langle x,(L-\lambda I) x_{0}\right\rangle \quad \text { para todo } x \in H .
$$

Assim, $(L-\lambda I) x_{0}=0$, contradizendo o fato de que $\|(L-\lambda I) y\| \geq c\|y\|$ para todo $y \in H$. Portanto, $x_{0}=0$, isto é, $\operatorname{Im}(L-\lambda I)^{\perp}=\{0\}$. Este fato prova que $\operatorname{Im}(L-\lambda I)=H$ e $L-\lambda I$ é sobrejetor. Em conclusão, se $\lambda<m$, então $\lambda \in \rho(L)$.

Analogamente podemos provar que, se $\lambda=M+c$, onde $c>0$, então $\lambda \in \rho(L)$. Daí, se $\lambda>M, \lambda \in \rho(L)$.

Consequentemente, $\sigma(L) \subseteq[m, M]$.

Definição 3.3.10. Sejam $L \in L(H)$ um operador auto-adjunto e $A$ um subconjunto de $H$. Dizemos que $L$ é positivo (negativo) em $A$, se $\langle L x, x\rangle>0(\langle L x, x\rangle<0)$ para todo $x \in A$, $\operatorname{com} x \neq 0$.

Suponhamos que $H_{1}$ seja um subespaço de $H$ invariante por $L$. Se $L$ é positivo (negativo) em $H_{1}$ dizemos que $L$ é definido positivo (definido negativo) em $H_{1}$. Se $\langle L x, x\rangle \geq 0(\langle L x, x\rangle \leq 0)$ para todo $x \in H_{1}$, diremos que $L$ é não negativo (não positivo) em $H_{1}$. Quando $H_{1}=H$ e $L$ é definido positivo (negativo) em $H$, diremos que $L$ é definido positivo (definido negativo). Se $L$ e $T$ são dois operadores auto-adjuntos, usaremos a notação $L \geq T$ quando $L-T$ é um operador não negativo.

Vamos apresentar a decomposição de $H$ como soma direta do núcleo e dos espaços espectrais negativo e positivo de um operador auto-adjunto $L$. Para este fato faremos uso da raiz quadrada de um operador limitado não negativo.

Definição 3.3.11 (Raiz quadrada). Seja $L \in L(H)$ um operador não negativo. Uma raiz quadrada de $L$ é um operador $R \in L(H)$ tal que $R^{2}=L$. 
É claro que, se $R$ é uma raiz quadrada de um operador não negativo $L$, então $-R$ também é uma raiz quadrada de $L$. Em geral, um operador não negativo pode ter várias raízes quadradas. Mostraremos que todo operador não negativo possui uma única raiz quadrada não negativa. Para provar este fato precisaremos dos dois seguintes lemas, cujas provas podem ser encontradas, por exemplo, em [16], pág. 470, Teorema 9.3-1 e pág. 473, Teorema 9.3-3, respectivamente.

Lema 3.3.12 (Composição de operadores positivos). Se dois operadores auto-adjuntos $L$ e $T$ em $L(H)$ são não negativos e comutam, então a composição LT é não negativa.

Lema 3.3.13 (Sequência monótona). Seja $\left(L_{n}\right)_{n=1}^{\infty}$ uma sequência de operadores autoadjuntos em um espaço de Hilbert $H$ tal que

$$
L_{1} \leq L_{2} \leq \ldots \leq L_{n} \leq \ldots \leq T,
$$

onde $T$ é um operador auto-adjunto em $L(H)$. Suponha que qualquer $L_{j}$ comute com $T$ e com todo $L_{m}$. Então, existe $L \in L(H)$ tal que $\left(L_{n}\right)_{n=1}^{\infty}$ converge pontualmente a $L$, isto é, $L_{n} x \rightarrow L x$ para todo $x \in H$. O operador $L$ é auto-adjunto e satisfaz $L \leq T$.

Teorema 3.3.14 (Teorema da raiz quadrada). Todo operador não negativo $L \in L(H)$ possui uma raiz quadrada não negativa $R$, a qual é única. $O$ operador $R$ comuta com todo operador em $L(H)$ que comute com $L$.

Demonstração. Primeiro provaremos o teorema com a hipótese adicional $L \leq I$. Vejamos a existência. Se $L=0$, tomamos $R=0$. Suponhamos que $L \neq 0$. Consideremos a sequência $\left(R_{n}\right)_{n=1}^{\infty}$, onde $R_{0}=0$ e

$$
R_{n+1}=R_{n}+\frac{1}{2}\left(L-R_{n}^{2}\right), \quad n=0,1,2, \ldots
$$

Provemos que $R_{n}$ converge pontualmente a um $R \in L(H)$ tal que $R^{2}=L$. É fácil ver que cada $R_{n}$ é um polinômio em $L$, isto é, $R_{n}=p_{n}(L)$, onde $p_{n}: \mathbb{K} \rightarrow \mathbb{K}$ é um polinômio. Assim, todos os $R_{n}$ são auto-adjuntos e todos comutam entre si. Além disso, observe que, se $M \in L(H)$ comuta com $L$, então $M$ comuta com cada $R_{n}$.

Mostremos que $R_{n} \leq I$ para todo $n$. De fato, para $n=0, R_{0}=0 \leq I$. Seja $n>0$ dado. Já que $I-R_{n-1}$ é auto-adjunto,

$$
\left\langle\left(I-R_{n-1}\right)^{2} x, x\right\rangle=\left\langle\left(I-R_{n-1}\right) x,\left(I-R_{n-1}\right) x\right\rangle \geq 0 \text { para todo } x \in H,
$$

isto é, $\left(I-R_{n-1}\right)^{2} \geq 0$. Como, por hipótese, $I-L \geq 0$, de (3.3.4) obtemos

$$
\begin{aligned}
0 & \leq \frac{1}{2}\left(I-R_{n-1}\right)^{2}+\frac{1}{2}(I-L)=\frac{1}{2}\left(I-2 R_{n-1}-R_{n-1}^{2}\right)+\frac{1}{2}(I-L) \\
& =I-R_{n-1}-\frac{1}{2}\left(L-R_{n-1}^{2}\right)=I-R_{n} .
\end{aligned}
$$


Consequentemente, $R_{n} \leq I$.

Agora vejamos que $R_{n} \leq R_{n+1}$ para todo $n \in \mathbb{N}$. Para este fim, usaremos indução sobre $n$. Se $n=0,0=R_{0} \leq R_{1}=\frac{1}{2} L$. Mostremos que, se $R_{n-1} \leq R_{n}$ para um $n$ fixado, então $R_{n} \leq R_{n+1}$. Dado que $R_{n} R_{n-1}=R_{n-1} R_{n}$, de (3.3.4) se segue

$$
\begin{aligned}
R_{n+1}-R_{n} & =R_{n}+\frac{1}{2}\left(L-R_{n}^{2}\right)-R_{n-1}-\frac{1}{2}\left(L-R_{n-1}^{2}\right) \\
& =R_{n}-\frac{1}{2} R_{n}^{2}-R_{n-1}+\frac{1}{2} R_{n-1}^{2} \\
& =R_{n}\left(I-\frac{1}{2} R_{n}\right)-R_{n-1}\left(I-\frac{1}{2} R_{n-1}\right) \\
& =R_{n}\left(I-\frac{1}{2} R_{n}\right)-\frac{1}{2} R_{n} R_{n-1}-R_{n-1}\left(I-\frac{1}{2} R_{n-1}\right)+\frac{1}{2} R_{n-1} R_{n} \\
& =R_{n}\left[I-\frac{1}{2}\left(R_{n}+R_{n-1}\right)\right]-R_{n-1}\left[I-\frac{1}{2}\left(R_{n-1}+R_{n}\right)\right] \\
& =\left(R_{n}-R_{n-1}\right)\left[I-\frac{1}{2}\left(R_{n}+R_{n-1}\right)\right] .
\end{aligned}
$$

Por hipótese, $R_{n}-R_{n-1} \geq 0$ e $I-\frac{1}{2}\left(R_{n}+R_{n-1}\right) \geq 0$. Além disso, dado que $R_{n}-R_{n-1}$ e $I-\frac{1}{2}\left(R_{n}+R_{n-1}\right)$ comutam, se segue do Lema 3.3.12 que

$$
\left(R_{n}-R_{n-1}\right)\left[I-\frac{1}{2}\left(R_{n}+R_{n-1}\right)\right] \geq 0
$$

isto é, $R_{n+1}-R_{n} \geq 0$.

Acima se provou que $\left(R_{n}\right)_{n=1}^{\infty}$ é monótona e $R_{n} \leq I$. Assim, o Lema 3.3.13 implica que existe um operador auto-adjunto $R \in L(H)$ tal que $R_{n} x \rightarrow R x$ para todo $x \in H$. De (3.3.4) temos

$$
\frac{1}{2}\left(L x-R_{n}^{2} x\right)=R_{n+1} x-R_{n} x \rightarrow 0 \text { quando } n \rightarrow \infty .
$$

Portanto, $L x-R^{2} x=0$ para todo $x \in H$. Este fato implica que $L=R^{2}$. Além disso, $0=R_{0} \leq R_{n}$ para todo $n$, isto é, $\left\langle R_{n} x, x\right\rangle \geq 0$ para todo $x \in H$. Consequentemente, $\langle R x, x\rangle \geq 0$ para todo $x \in H$ pela continuidade do produto interno. Logo, $R \geq 0$.

Seja $S \in L(H)$ tal que $S L=L S$. Então, $R_{n} S=S R_{n}$, pois todo operador que comuta com $L$ comuta com $R_{n}$. Daí, $R_{n} S x=S R_{n} x$ para todo $x \in H$. Fazendo $n \rightarrow \infty$, temos $R S=S R$.

Provemos agora a unicidade. Sejam $R$ e $T$ raízes quadradas não negativas do operador $L$. Então, $R^{2}=T^{2}=L$. Além disso,

$$
T L=T T^{2}=T^{2} T=L T,
$$

isto é, $T$ comuta com $L$. Portanto, como se provou acima, $R T=T R$. Para $x \in H$, tomemos $y=(R-T) x$. Assim, $\langle R y, y\rangle \geq 0$ e $\langle T y, y\rangle \geq 0$, pois $R$ e $T$ são não negativos. 
Já que $T R=R T$ e $R^{2}=T^{2}$, obtemos

$$
\langle R y, y\rangle+\langle T y, y\rangle=\langle(R+T) y, y\rangle=\langle(R+T)(R-T) x, y\rangle=\left\langle\left(R^{2}-T^{2}\right) x, y\right\rangle=0 .
$$

Logo,

$$
\langle R y, y\rangle=\langle T y, y\rangle=0 .
$$

Dado que $R$ é não negativo, $R$ possui uma raiz quadrada não negativa $P$. Assim,

$$
0=\langle R y, y\rangle=\left\langle P^{2} y, y\right\rangle=\langle P y, P y\rangle=\|P y\|^{2},
$$

isto é, $P y=0$. Daí,

$$
R y=P^{2} y=P(P y)=0 .
$$

Analogamente podemos provar que $T y=0$. Então, $(R-T) y=0$. Como $y=(R-T) x$, temos

$$
\|R x-T x\|^{2}=\langle(R-T) x,(R-T) x\rangle=\left\langle(R-T)^{2} x, x\right\rangle=\langle(R-T) y, x\rangle=0 .
$$

Portanto, $R x-T x=0$ para todo $x \in H$, isto é, $R=T$.

Agora provemos o caso geral. Podemos supor que $L \neq 0$. Da desigualdade de Cauchy-Schwarz se segue

$$
\langle L x, x\rangle \leq\|L x\|\|x\| \leq\|L\|\|x\|^{2} .
$$

Logo, tomando $Q=(1 /\|L\|) L$, obtemos

$$
\langle Q x, x\rangle \leq\|x\|^{2}=\langle I x, x\rangle,
$$

isto é, $Q \leq I$. Como foi provado acima, $Q$ tem uma única raiz quadrada não negativa $B$. Portanto,

$$
\left(\|L\|^{1 / 2} B\right)^{2}=\|L\| B^{2}=\|L\| Q=L,
$$

isto é, $\|L\|^{1 / 2} B$ é uma raiz quadrada não negativa de $L$. A unicidade da raiz quadrada de $L$ se segue da unicidade da raiz quadrada de $Q$.

Seja $L \in L(H)$ um operador não negativo e $R$ a raiz quadrada não negativa de $L$. Vejamos que

$$
\operatorname{Ker} R=\operatorname{Ker} L \quad \text { e } \quad \overline{\operatorname{Im} R}=\overline{\operatorname{Im} L} .
$$

De fato, é claro que Ker $R \subseteq \operatorname{Ker} L$. Suponhamos que $x \in \operatorname{Ker} L$. Assim,

$$
0=\langle L x, x\rangle=\langle R R x, x\rangle=\langle R x, R x\rangle=\|R x\|^{2} .
$$

Logo, $R x=0$. Portanto, Ker $L \subseteq \operatorname{Ker} R$. Consequentemente, $\operatorname{Ker} R=\operatorname{Ker} L$.

Por outro lado, das Proposições 3.1.5 e 3.2.9 se segue

$$
\overline{\operatorname{Im} L}=\overline{\operatorname{Im} L^{*}}=(\operatorname{Ker} L)^{\perp}=(\operatorname{Ker} R)^{\perp}=\overline{\operatorname{Im} R^{*}}=\overline{\operatorname{Im} R} .
$$


Além disso, observe que $L$ é definido positivo em $\overline{\operatorname{Im} L}$. De fato, tomemos $x \in$ $\overline{\operatorname{Im} L}=\overline{\operatorname{Im} R} \operatorname{com} x \neq 0$. Assim, $R x \neq 0$. Logo,

$$
\langle L x, x\rangle=\left\langle R^{2} x, x\right\rangle=\langle R x, R x\rangle=\|R x\|>0 .
$$

É fácil ver que, se $L \in L(H)$ é um isomorfismo e $R \in L(H)$ é um operador tal que $R^{2}=L$, então $R$ é um isomorfismo. Consequentemente, a raiz quadrada não negativa de um isomorfismo não negativo é um isomorfismo.

Observação 3.3.15. Sejam $L \in L(H)$ um operador não negativo e $R$ sua raiz quadrada não negativa. De (3.3.5) se segue que $L$ é definido positivo em $\overline{\operatorname{Im} L}$.

Por outro lado, seja $L \in L(H)$ um operador não positivo. Assim, $-L$ é não negativo. Pelo fato acima, $-L$ é definido positivo em $\overline{\operatorname{Im} L}$. Daí, $L$ é definido negativo em $\overline{\operatorname{Im} L}$.

Podemos agora enunciar o teorema que apresenta a existência e a unicidade da decomposição polar de um operador linear limitado.

Teorema 3.3.16 (Teorema da decomposição polar). Seja L um operador em $L(H)$ (não necessariamente não negativo). Então, existem dois únicos operadores $R$ e $O$ em $L(H)$ com as seguintes propriedades:

i. $R$ é não negativo,

ii. O é um operador ortogonal de $\operatorname{Im} R$ em $\operatorname{Im} L$,

iii. $\operatorname{Ker} O=\operatorname{Ker} R=\operatorname{Ker} L e$

iv. $L=O R$.

Demonstração. Vejamos primeiro a existência. De fato, é fácil ver que o operador $L^{*} L$ é não negativo em $H$. Do Teorema 3.3 .14 se segue que existe um único operador não negativo $R \in L(H)$ tal que $R^{2}=L^{*} L$. Para $x_{1}, x_{2} \in H$,

$$
\left\langle L x_{1}, L x_{2}\right\rangle=\left\langle L^{*} L x_{1}, x_{2}\right\rangle=\left\langle R^{2} x_{1}, x_{2}\right\rangle=\left\langle R x_{1}, R x_{2}\right\rangle .
$$

Assim,

$$
\|L x\|=\|R x\| \quad \text { para todo } x \in H .
$$

Este fato prova que

Ker $R=\operatorname{Ker} L$.

Para cada $y \in \operatorname{Im} R$, existe $x \in H$ tal que $y=R x$. Ponhamos

$$
O y=O R x=L x .
$$

Vejamos que $O$ define um operador ortogonal de $\operatorname{Im} R$ a $\operatorname{Im} L$. De fato, primeiro provemos que $O$ está bem definido. Suponhamos que, para algum $y \in \operatorname{Im} R, y=$ 
$R x_{1}=R x_{2}$. Daí, $R\left(x_{1}-x_{2}\right)=0$. Dado que $\operatorname{Ker} R=\operatorname{Ker} L$, então $L\left(x_{1}-x_{2}\right)=0$. Assim,

$$
O y=L x_{1}=L x_{2} .
$$

Consequentemente, $O$ está bem definido sobre a imagem de $R$.

O operador $O$ é linear, pois, se $c \in \mathbb{K}$ e $y_{1}, y_{2} \in \operatorname{Im} R$, então $y_{1}=R x_{1}$ e $y_{2}=R x_{2}$, onde $x_{1}, x_{2} \in H$. Logo,

$O\left(c y_{1}+y_{2}\right)=O\left(c R x_{1}+R x_{2}\right)=O R\left(c x_{1}+x_{2}\right)=L\left(c x_{1}+x_{2}\right)=c L x_{1}+L x_{2}=c O y_{1}+O y_{2}$.

Além disso, de (3.3.6) temos $\left\langle R x_{1}, R x_{2}\right\rangle=\left\langle L x_{1}, L x_{2}\right\rangle$. Daí,

$$
\left\langle O y_{1}, O y_{2}\right\rangle=\left\langle O R x_{1}, O R x_{2}\right\rangle=\left\langle L x_{1}, L x_{2}\right\rangle=\left\langle R x_{1}, R x_{2}\right\rangle=\left\langle y_{1}, y_{2}\right\rangle .
$$

Como $O$ é sobrejetor, a Proposição 3.2.6 implica que $O$ é um operador ortogonal de $\operatorname{Im} R$ a $\operatorname{Im} L$.

Se segue do Corolário 1.1.5 e a Proposição 3.2.8 que $O$ pode ser estendido a um operador ortogonal em $\overline{\operatorname{Im} R}$ sobre $\overline{\operatorname{Im} L}$. O operador $O$ ainda pode ser estendido a um operador em $L(H)$, o qual pode ser denotado de novo por $O$, tomando $O x=0$ para todo $x \in(\operatorname{Im} R)^{\perp}=\operatorname{Ker} R$. Assim, a igualdade (3.3.8) implica que

$$
L x=O R x \quad \text { para todo } x \in H .
$$

É claro que

$$
\text { Ker } O=\operatorname{Ker} R=\operatorname{Ker} L \text {. }
$$

Vejamos agora a unicidade. De fato, suponhamos que $L=O_{1} R_{1}$, onde $R_{1}$ é não negativo e $O_{1}$ é ortogonal na imagem de $R_{1}$. Assim,

$$
L^{*}=R_{1}^{*} O_{1}^{*}=R_{1} O_{1}^{*} .
$$

Dado que $O_{1}$ é ortogonal na imagem de $R_{1}$, então

$$
O_{1}^{*} O_{1} R_{1}=R_{1} .
$$

Logo, $L^{*} L=R_{1} O_{1}^{*} O_{1} R_{1}=R_{1}^{2}$. Daí, $R_{1}$ é a raiz quadrada não negativa de $L^{*} L$, a qual é única pelo Teorema 3.3.14. Portanto, $R_{1}=R$. A igualdade (3.3.7) determina a unicidade do operador $O$ em $\overline{\operatorname{Im} R}$ e assim em $H$, pois, por hipótese, $O=0 \mathrm{em}$ $(\overline{\operatorname{Im} R})^{\perp}=\operatorname{Ker} R$. Consequentemente, a decomposição $L=O R$ é única.

Definição 3.3.17. A decomposição $L=O R$, dada no teorema anterior, é chamada a decomposição polar do operador $L$. 
Suponhamos agora que $L$ seja auto-adjunto e que $O R$ seja a decomposição polar de $L$. Dado que $\operatorname{Ker} L=\operatorname{Ker} R$, das Proposições 3.1.5 e 3.2.9 temos

$$
\overline{\operatorname{Im} L}=\overline{\operatorname{Im} L^{*}}=(\operatorname{Ker} L)^{\perp}=(\operatorname{Ker} R)^{\perp}=\overline{\operatorname{Im} R^{*}}=\overline{\operatorname{Im} R} .
$$

Como $R$ é não negativo, da Observação 3.3 .15 concluímos que $R$ é definido positivo em $\overline{\operatorname{Im} R}=\overline{\operatorname{Im} L}$. Além disso, já que $\operatorname{Ker} O=\operatorname{Ker} L$, se segue que

$$
\operatorname{Ker} O^{*}=\operatorname{Ker} L^{*}=\operatorname{Ker} L=\operatorname{Ker} R \text {. }
$$

Provemos que $O=O^{*}$. De fato, se $x \in \operatorname{Ker} R$,

$$
0=O R x=R O x
$$

pois $\operatorname{Ker} O=\operatorname{Ker} R$ pelo teorema anterior. É fácil ver que $O R O^{*}$ é não negativo, pois $R$ é não negativo. Agora, de (3.3.9) se segue $O^{*} O R=R$. Assim,

$$
\left(O R O^{*}\right)^{2}=O R O^{*} O R O^{*}=O R R O^{*}=L L^{*}=L^{2}=L^{*} L,
$$

isto é, $O R O^{*}$ é uma raiz quadrada não negativa do operador $L^{*} L$. Dado que $R$ é a raiz quadrada não negativa de $L^{*} L$, temos $O R O^{*}=R$ pela unicidade da raiz quadrada. Daí, $R O=O R O^{*} O$. Logo, $R O=O R$ na imagem de $R(O$ é ortogonal em $\operatorname{Im} R$ ). Portanto,

$$
R O x=O R x \quad \text { para todo } x \in \overline{\operatorname{Im} R} .
$$

Se segue de (3.3.10) e (3.3.11) que

$$
R O x=O R x \quad \text { para todo } x \in H .
$$

Consequentemente,

$$
L=L^{*}=(O R)^{*}=(R O)^{*}=O^{*} R .
$$

Daí, $L=O^{*} R$, o qual prova que $O=O^{*}$ pela unicidade da decomposição polar.

Observe que,

$$
\text { se } x \in \overline{\operatorname{Im} L}, \quad O^{2} x=O^{*} O x=x \quad \text { e } \quad \text { se } x \in(\overline{\operatorname{Im} L})^{\perp}, \quad O x=0 .
$$

Vejamos que qualquer $x \in \overline{\operatorname{Im} L}$ pode ser escrito de modo único como

$$
x=x_{+}+x_{-}, \quad \text { onde } O x_{+}=x_{+} \text {e } O x_{-}=-x_{-} .
$$

De fato, é fácil ver que, para $x \in H, x_{+}=(I+O) x / 2$ e $x_{-}=(I-O) x / 2$ satisfazem as condições acima. Suponhamos que $x=x_{+}+x_{-}=z_{+}+z_{-}$, onde

$$
O x_{+}=x_{+}, \quad O x_{-}=-x_{-}, \quad O z_{+}=z_{+} \quad \text { e } \quad O z_{-}=-z_{-} .
$$


Assim, $x_{+}-z_{+}=z_{-}-x_{-}$. Logo

$$
x_{+}-z_{+}=O\left(x_{+}-z_{+}\right)=O\left(z_{-}-x_{-}\right)=-\left(z_{-}-x_{-}\right) .
$$

Portanto, $x_{+}-z_{+}=z_{-}-x_{-}=0$.

Sejam $H_{ \pm}(L)$ os subespaços de $\overline{\operatorname{Im} L}$ consistentes de todos os $x$ tais que $O x= \pm x$, isto é,

$$
H_{+}(L)=\{x \in H: O x=x\} \quad \text { e } \quad H_{-}(L)=\{x \in H: O x=-x\}
$$

Daí,

$$
\overline{\operatorname{Im} L}=H_{+}(L) \oplus H_{-}(L) .
$$

Vejamos que os subespaços $H_{+}(L)$ e $H_{-}(L)$ são fechados. Seja $\left(x_{n}\right)_{n=1}^{\infty}$ uma sequência em $H_{+}(L)$ convergente a $x \in H$. Então, $O x_{n}=x_{n}$ para todo $n \in \mathbb{N}$. Agora,

$\|O x-x\|=\left\|O x-O x_{n}+O x_{n}-x\right\|=\left\|O x-O x_{n}+x_{n}-x\right\| \leq\left\|O x-O x_{n}\right\|+\left\|x_{n}-x\right\|$.

Como $x_{n} \rightarrow x$, temos que $O x_{n} \rightarrow O x$. Consequentemente, $O x=x$ e $x \in H_{+}(L)$. Analogamente, $H_{-}(L)$ é fechado.

Provemos agora que $H_{+}(L)$ e $H_{-}(L)$ são ortogonais. De fato, se $x_{+} \in H_{+}(L)$ e $x_{-} \in H_{-}(L)$, então $O x_{+}=x_{+}$e $O x_{-}=-x_{-}$. Daí,

$$
\left\langle x_{+}, x_{-}\right\rangle=\left\langle x_{+}, O^{*} O x_{-}\right\rangle=\left\langle O x_{+}, O x_{-}\right\rangle=\left\langle x_{+},-x_{-}\right\rangle=-\left\langle x_{+}, x_{-}\right\rangle .
$$

Assim, $\left\langle x_{+}, x_{-}\right\rangle=0$, o que prova que $H_{+}(L)$ e $H_{-}(L)$ são ortogonais.

Dado que $\overline{\operatorname{Im} L}=H_{+}(L) \oplus H_{-}(L)$ e $\operatorname{Ker} L=(\overline{\operatorname{Im} L})^{\perp}$, obtemos a decomposição

$$
H=H_{+}(L) \oplus H_{-}(L) \oplus \operatorname{Ker} L,
$$

sendo os três subespaços dois a dois ortogonais.

Agora, como $O=O^{*}$ e $L=O R=R O$, temos

$$
O L=O^{*} L=R=L O^{*}=L O .
$$

Logo,

$$
O L x=L O x=L x \text { se } x \in H_{+}(L) \quad \text { e } \quad O L x=L O x=-L x \text { se } x \in H_{-}(L) .
$$

Daí, os subespaços $H_{+}(L)$ e $H_{-}(L)$ são invariantes por $L$.

O operador $L$ também comuta com $R$, pois

$$
R L=R R O=L^{2} O=O L^{2}=O R R=L R .
$$

Por outro lado, para $x \in H_{+}(L)$, temos

$$
L x=L O x=R x .
$$


Como $R$ é definida positiva em $\operatorname{Im} L$, então $L$ é definida positiva em $H_{+}(L)$. Agora, se $x \in H_{-}(L)$, então

$$
L x=-L O x=-R x .
$$

Portanto, $L$ é definida negativa em $H_{-}(L)$.

Observe que as projeções ortogonais sobre os espaços $H_{+}(L), H_{-}(L)$ e $\operatorname{Ker} L$ são, respectivamente,

$$
P_{H_{+}(L)}=\frac{1}{2}\left(O^{2}+O\right), \quad P_{H_{-}(L)}=\frac{1}{2}\left(O^{2}-O\right) \quad \text { e } \quad P_{\text {Ker } L}=I-O^{2} .
$$

De fato, se $x \in H$, então $x=x_{+}+x_{-}+x_{0}$, onde $x_{+} \in H_{+}(L), x_{-} \in H_{-}(L)$ e $x_{0} \in \operatorname{Ker} L$. Logo, de (3.3.13) temos

$$
\frac{1}{2}\left(O^{2}+O\right) x=\frac{1}{2}\left(O^{2}\left(x_{+}+x_{-}+x_{0}\right)+O\left(x_{+}+x_{-}+x_{0}\right)\right)=\frac{1}{2}\left(x_{+}+x_{-}+x_{+}-x_{-}\right)=x_{+} .
$$

Dado que $O$ é auto-adjunto, $P_{H_{+}(L)}$ é auto-adjunto. Consequentemente, pela Proposição 3.2.13, $P_{H_{+}(L)}$ é a projeção ortogonal sobre $H_{+}(L)$, pois $P_{H_{+}(L)}$ é auto-adjunto e $P_{H_{+}(L)}^{2}=P_{H_{+}(L)}$.

Analogamente podemos provar que $P_{H_{-}(L)}$ e $P_{\operatorname{Ker} L}$ são as projeções ortogonais sobre $H_{-}(L)$ e $P_{\text {Ker } L}$, respectivamente. Na Seção 3 do Capítulo 4 veremos outra expressão para estas projeções e, além disso, mostraremos que elas dependem continuamente do operador.

Provaremos que, para um operador auto-adjunto $L \in L(H)$, a decomposição dada em (3.3.14) é única no seguinte sentido: Se existir dois subespaços ortogonais $H_{1}$ e $H_{2}$ tais que a soma $H=H_{1} \oplus H_{2} \oplus \operatorname{Ker} L$ é ortogonal e $L$ é definido positivo em $H_{1}$ e definido negativo em $H_{2}$, então $H_{+}(L)=H_{1}$ e $H_{-}(L)=H_{2}$. Antes de provar este fato, vejamos primeiro o seguinte lema.

Lema 3.3.18. Sejam $L \in L(H)$ um operador auto-adjunto e $O R$ a decomposição polar de $L$. Se $T \in L(H)$ comuta com $L$, então $T$ comuta com $R$ e $O$.

Demonstração. O operador $T$ comuta com o operador resolvente de $L$, pois, se $\lambda \in$ $\rho(L)$, então

$$
\begin{aligned}
(L-\lambda I)^{-1} T & =(L-\lambda I)^{-1} T(L-\lambda I)(L-\lambda I)^{-1} \\
& =(L-\lambda I)^{-1}(L-\lambda I) T(L-\lambda I)^{-1} \\
& =T(L-\lambda I)^{-1} .
\end{aligned}
$$

Analogamente, $T$ comuta com a resolvente de $L^{2}$. Assim, pelo Teorema 3.3.14, $T$ comuta com a raiz quadrada não negativa de $L^{2}$, que denotamos por $R$.

Para provar que $T$ comuta com $O$, observe que

$$
T O R=T L=L T=O R T=O T R,
$$


isto é,

$$
T O x=O T x \quad \text { para todo } x \in \overline{\operatorname{Im} R}=\overline{\operatorname{Im} L} .
$$

Por outro lado, se $x \in \operatorname{Ker} L$, temos $T O x=0$, pois $\operatorname{Ker} L=\operatorname{Ker} O$. Dado que $T L=$ $L T$, então $L T x=0$, isto é, $T x \in \operatorname{Ker} L=\operatorname{Ker} O$. Daí, $O T x=0$. Consequentemente,

$$
O T x=T O x \quad \text { para todo } x \in \operatorname{Ker} L .
$$

De (3.3.15) e (3.3.16) temos $T O=O T$.

Consideremos, de novo, um operador auto-adjunto $L \in L(H)$. Vejamos que, se $H^{\prime}$ é um subespaço de $H$ invariante por $L$ e $\langle L x, x\rangle \geq 0$ para todo $x \in H^{\prime}$, então $H^{\prime}$ é um subespaço de $H_{+}(L) \oplus \operatorname{Ker} L$. De fato, seja $O R$ a decomposição polar de $L$. Denotemos por $P^{\prime}$ a projeção ortogonal sobre $H^{\prime}$. Já que $H^{\prime}$ é invariante por $L, P^{\prime}$ comuta com $L$. Se segue do Lema 3.3.18 que $P^{\prime}$ comuta com $O$. Assim, de (3.3) temos que $P^{\prime}$ comuta com $P_{H_{-}(L)}$. O Corolário 3.2.14 mostra que $P^{\prime} P_{H_{-}(L)}$ é a projeção sobre $H^{\prime} \cap H_{-}(L)$. É claro que a interseção $H^{\prime} \cap H_{-}(L)$ é $\{0\}$, pois $L$ é definida negativa em $H_{-}(L)$ e é não negativa em $H^{\prime}$. Logo, $P^{\prime} P_{H_{-}(L)}=P_{H_{-}(L)} P^{\prime}=0$. Assim, se $x \in H^{\prime}$, então

$$
P_{H_{-}(L)} x=P_{H_{-}(L)} P^{\prime} x=0,
$$

isto é, $x \in\left(H_{-}(L)\right)^{\perp}=H_{+}(L) \oplus \operatorname{Ker} L$. Este fato prova que $H^{\prime} \subseteq H_{+}(L) \oplus \operatorname{Ker} L$.

Analogamente, se $\langle L x, x\rangle \leq 0$ para todo $x \in H^{\prime}$, então $H^{\prime}$ é um subespaço de $H_{-}(L) \oplus \operatorname{Ker} L=\left(H_{+}(L)\right)^{\perp}$.

Os fatos mostrados acima provam o seguinte teorema.

Teorema 3.3.19 (Teorema espectral). Seja $L \in L(H)$ auto-adjunto. Existe uma única decomposição

$$
H=H_{+}(L) \oplus H_{-}(L) \oplus \operatorname{Ker} L
$$

tal que:

i. $\overline{\operatorname{Im} L}=H_{+}(L) \oplus H_{-}(L)$,

ii. $H_{+}(L)$ e $H_{-}(L)$ são subespaços fechados de $H$ invariantes por $L$,

iii. $H_{+}(L), H_{-}(L)$ e $\operatorname{Ker} L$ são dois a dois ortogonais e

iv. L é definido positivo em $H_{+}(L)$ e definido negativo em $H_{-}(L)$.

Definição 3.3.20. Para um operador auto-adjunto $L \in L(H)$, os subespaços $H_{+}(L)$ e $H_{-}(L)$ definidos acima são chamados de subespaço espectral positivo e subespaço espectral negativo de $L$, respectivamente. 
Suponhamos que $L$ seja um operador auto-adjunto em um espaço de Hilbert de dimensão finita $H$. Neste caso $\sigma(L)$ consiste dos auto-valores de $L$. É claro que $L$ é definida positiva no autoespaço gerado pelos autovetores que possuem autovalores positivos e que é definida negativa no autoespaço gerado pelos autovetores que possuem autovalores negativos. Não é difícil provar que $H_{+}(L)$ é gerado pelos autovetores que possuem autovalores positivos e que $H_{-}(L)$ é gerado pelos autovetores que possuem autovalores negativos.

Os subespaços espectrais negativo e positivo de um operador auto-adjunto $L$ em um espaço de Hilbert de dimensão infinita $H$ são uma generalização dos autoespaços. Neste caso, é fácil provar que o autoespaço gerado pelos autovetores com respectivos autovalores positivos é um subespaço de $H_{+}(L)$ e o autoespaço gerado pelos autovetores com respectivos autovalores negativos é um subespaço de $H_{-}(L)$.

Seja $L \in L(H)$ um operador auto-adjunto fixado. Dado que $H_{+}(L)$ e $H_{-}(L)$ são invariantes por $L$, podemos considerar as restricões

$$
L_{+}=\left.L\right|_{H_{+}(L)}: H_{+}(L) \rightarrow H_{+}(L) \quad \text { e } \quad L_{-}=L_{H_{-}(L)}: H_{-}(L) \rightarrow H_{-}(L)
$$

do operador L. Da Proposição 3.3.7 temos que

$$
\sigma(L)=\sigma\left(L_{+}\right) \cup \sigma\left(L_{-}\right) \cup \sigma\left(L_{0}\right),
$$

onde $L_{0}$ é a restrição de $L$ a $\operatorname{Ker} L$. Observamos que, se $\operatorname{Ker} L$ for nulo, $\sigma\left(L_{0}\right)$ é vazio; do contrario $\sigma\left(L_{0}\right)=0$.

Observação 3.3.21. Dado que $L_{+}$é definido positivo e $L_{-}$é definido negativo, seguese de (3.3.17) e da Proposição 3.3.9 que

$$
\sigma\left(L_{+}\right)=\sigma(L) \cap \mathbb{R}^{+}=\sigma^{+}(L) \quad \text { e } \quad \sigma\left(L_{-}\right)=\sigma(L) \cap \mathbb{R}^{-}=\sigma^{-}(L),
$$

isto é, o espectro de $L_{+}$corresponde à parte positiva do espectro de $L$ e o espectro de $L_{-}$corresponde à parte negativa do espectro de $L$.

\subsection{Operadores de Fredholm auto-adjuntos em es- paços de Hilbert}

O propósito desta seção é mostrar que, se $H$ é um espaço de Hilbert real, de dimensão infinita e separável, o conjunto $\Phi_{S}(H)$ dos operadores de Fredholm auto-adjuntos em $L(H)$ possui três componentes conexas que são:

i. O conjunto dos operadores essencialmente positivos, denotado por $\Phi_{S}^{+}(H)$, que consiste dos operadores em $\Phi_{S}(H)$ tais que seu subespaço espectral negativo tem dimensão finita. 
ii. O conjunto dos operadores essencialmente negativos, denotado por $\Phi_{S}^{-}(H)$, que consiste dos operadores em $\Phi_{S}(H)$ tais que seu subespaço espectral positivo tem dimensão finita.

iii. O conjunto dos operadores fortemente indefinidos, denotado por $\Phi_{S}^{i}(H)$, que consiste dos operadores com subespaços espectrais positivo e negativo de dimensão infinita.

Em particular, provaremos que $\Phi_{S}^{+}(H)$ e $\Phi_{S}^{-}(H)$ são convexos e que $\Phi_{S}^{i}(H)$ é conexo por caminhos. A não trivial prova destas conhecidas propriedades não se encontram facilmente na literatura. Decidimos portanto providenciá-la nesta seção.

Em símbolos, podemos escrever

$$
\Phi_{S}(H)=\Phi_{S}^{+}(H) \cup \Phi_{S}^{-}(H) \cup \Phi_{S}^{i}(H)
$$

É claro que $\Phi_{S}^{+}(H), \Phi_{S}^{-}(H)$ e $\Phi_{S}^{i}(H)$ são dois a dois disjuntos.

Os conjuntos $\Phi_{S}^{+}(H), \Phi_{S}^{-}(H)$ e $\Phi_{S}^{i}(H)$ são analogamente definidos quando $H$ é um espaço de Hilbert real não necessariamente separável ou quando $H$ tem dimensão finita. Observe que, se $H$ tem dimensão finita, então $\Phi_{S}^{+}(H)=\Phi_{S}^{-}(H)=L_{S}(H)$ e $\Phi_{S}^{i}(H)$ é vazio.

Além dos fatos anteriores, apresentaremos algumas propriedades dos operadores de Fredholm auto-adjuntos nos espaços de Hilbert reais. Nesta seção, se não se diz o contrario, $H$ denotará um espaço de Hilbert real de dimensão finita ou infinita.

De (3.2.5) temos que, se $L \in L(H)$, então $H=\overline{\operatorname{Im} L^{*}} \oplus \operatorname{Ker} L$. No capítulo anterior provamos que a imagem de um operador de Fredholm é fechada. Consequentemente, se $L$ é um operador de Fredholm auto-adjunto,

$$
H=\operatorname{Im} L \oplus \operatorname{Ker} L
$$

A seguinte proposição é uma outra consequência de (3.2.5).

Proposição 3.4.1. Seja L um operador auto-adjunto. Suponhamos que a imagem de $L$ seja fechada e que $\operatorname{dim} \operatorname{Ker} L<\infty$. Então, $L$ é um operador de Fredholm e ind $L=0$.

Demonstração. Dado que $\operatorname{Im} L$ é fechada, de (3.2.5) temos

$$
H=\overline{\operatorname{Im} L^{*}} \oplus \operatorname{Ker} L=\operatorname{Im} L \oplus \operatorname{Ker} L .
$$

Logo,

$$
\operatorname{dim} \operatorname{Ker} L=\operatorname{dim}(\operatorname{Im} L)^{\perp}=\operatorname{dim} \operatorname{coKer} L .
$$

Este fato prova que $L$ é de Fredholm e que ind $L=0$.

Observação 3.4.2. Da proposição anterior se segue que o índice de qualquer operador de Fredholm auto-adjunto é 0. Portanto, se $L$ é um operador de Fredholm auto-adjunto, temos que $\operatorname{Ker} L=\{0\}$ se, e somente se, $\operatorname{Im} L=H$. 
Seja $E$ um espaço de Banach real. Observe que, se $L \in L(E)$ é um operador de Fredholm de índice 0 (não necessariamente auto-adjunto), então $0 \in \rho(L)$ ou 0 é um autovalor de $L$. De fato, suponhamos que $0 \in \sigma(L)$. Como ind $L=0$,

$$
\operatorname{dim} \operatorname{Ker} L=\operatorname{dim} \operatorname{coKer} L \text {. }
$$

Assim, dado que $L$ é não inversível, pois $0 \in \sigma(L)$, e $E$ é de Banach, se segue que $L$ é não injetor ou não é sobrejetor. Em ambos os casos temos que $\operatorname{dim} \operatorname{Ker} L>0$, isto é, 0 é um autovalor de $L$.

Vejamos algumas outras propriedades que possuem os operadores de Fredholm autoadjuntos.

Proposição 3.4.3. Seja $L \in L(H)$ um operador de Fredholm auto-adjunto. Então, 0 não é um ponto de acumulação de $\sigma(L)$.

Demonstração. De fato, de (3.4.1) temos que $H=\operatorname{Im} L \oplus \operatorname{Ker} L$ (Ker $L$ pode ser $\{0\}$ ). Seja

$$
L=\left(\begin{array}{cc}
L_{1} & 0 \\
0 & 0
\end{array}\right)
$$

a matriz de operadores de $L$ a respeito da decomposição de $H$. É claro que $L_{1}$ é um automorfismo de $\operatorname{Im} L$. Como $G L(\operatorname{Im} L)$ é aberto em $L(\operatorname{Im} L)$, existe um $\varepsilon>0$ tal que, se $|\lambda|<\varepsilon$, então $L_{1}-\left.\lambda I\right|_{\operatorname{Im} L}$ é inversível. Observe que, para $|\lambda|<\varepsilon$ e $\lambda \neq 0$,

$$
(L-\lambda I)^{-1}=\left(\begin{array}{cc}
L_{1}-\left.\lambda I\right|_{\operatorname{Im} L} & 0 \\
0 & -\left.\lambda I\right|_{\operatorname{Ker} L}
\end{array}\right)^{-1}=\left(\begin{array}{cc}
\left(L_{1}-\left.\lambda I\right|_{\operatorname{Im} L}\right)^{-1} & 0 \\
0 & -\left.\frac{1}{\lambda} I\right|_{\operatorname{Ker} L}
\end{array}\right) .
$$

Logo, $(L-\lambda I)^{-1} \in L(H)$. Este fato prova que existe uma vizinhança $V$ de 0 tal que $L-\lambda I$ é inversível para todo $\lambda \in V \operatorname{com} \lambda \neq 0$. Assim, 0 não é ponto de acumulação de $\sigma(L)$.

Observação 3.4.4. Outra propriedade dos operadores de Fredholm auto-adjuntos é que, para todo operador de Fredholm auto-adjunto $L$, existe um operador auto-adjunto com imagem de dimensão finita $K$ tal que $L+K$ é um isomorfismo auto-adjunto. Este fato se obtém tomando $K=P_{\operatorname{Ker} L}$, a projeção ortogonal sobre o $\operatorname{Ker} L$, na Proposição 2.1.7 e aplicando o Teorema da aplicação aberta (lembramos que a Proposição 2.1.7 atua em espaços vetoriais).

Observe que, se $L$ é um operador de Fredholm auto-adjunto, então

$$
\operatorname{Im} L=H_{+}(L) \oplus H_{-}(L),
$$

onde $H_{+}(L)$ e $H_{-}(L)$ são os subespaços espectrais positivo e negativo de $L$, respectivamente (veja-se o Teorema 3.3.19). Consequentemente, dado que $H_{+}(L)$ e $H_{-}(L)$ são invariantes por $L$, as restricões

$$
L_{+}=\left.L\right|_{H_{+}(L)}: H_{+}(L) \rightarrow H_{+}(L) \quad \text { e } \quad L_{-}=L_{H_{-}(L)}: H_{-}(L) \rightarrow H_{-}(L)
$$


de $L$ são isomorfismos. Destacamos que, se $L$ não for de Fredholm, temos que $\overline{\operatorname{Im} L}=$ $H_{+}(L) \oplus H_{-}(L)$; neste caso, $L_{-}$e $L_{+}$são injetores, mas não necessariamente são isomorfismos.

Para provar que os espaços $\Phi_{S}^{+}(H)$ e $\Phi_{S}^{-}(H)$ são convexos, primeiro vejamos o seguinte resultado conhecido na teoria dos operadores em espaços de Hilbert, do qual damos a prova por razões de precisão.

Lema 3.4.5. Suponhamos que H seja um espaço de Hilbert real ou complexo. Se $L: H \rightarrow H$ é um isomorfismo definido positivo, então existe $l>0$ tal que

$$
\inf _{\|x\|=1}\langle L x, x\rangle \geq l
$$

Demonstração. De fato, sejam $x, y \in H$ e tais que $\langle L x, y\rangle \neq 0$. Para $a \in \mathbb{R}$, tomemos $w_{a}=x+a\langle L x, y\rangle y$. Como $L$ é definido positivo, temos

$$
\begin{aligned}
0 & \leq\left\langle L w_{a}, w_{a}\right\rangle \\
& =\langle L(x+a\langle L x, y\rangle y), x+a\langle L x, y\rangle y\rangle \\
& =\langle L x, x\rangle+\langle L x, a\langle L x, y\rangle y+\langle L(a\langle L x, y\rangle y), x\rangle+\langle L(a\langle L x, y\rangle y), a\langle L x, y\rangle y\rangle \\
& =\langle L x, x\rangle+a \overline{\langle L x, y\rangle}\langle L x, y\rangle+a\langle L x, y\rangle\langle L y, x\rangle+a^{2}\langle L x, y\rangle \overline{\langle L x, y\rangle}\langle L y, y\rangle \\
& =\langle L x, x\rangle+a \overline{\langle L x, y\rangle}\langle L x, y\rangle+a\langle L x, y\rangle \overline{\langle L x, y\rangle}+a^{2}\langle L x, y\rangle \overline{\langle L x, y\rangle}\langle L y, y\rangle .
\end{aligned}
$$

Portanto, para todo $a \in \mathbb{R}$,

$$
\langle L x, x\rangle+2 a \overline{\langle L x, y\rangle}\langle L x, y\rangle+a^{2}\langle L x, y\rangle \overline{\langle L x, y\rangle}\langle L y, y\rangle \geq 0 .
$$

Consequentemente, tomando a parte esquerda da desigualdade anterior como um polinômio em $a$, temos que o discriminante deste polinômio é menor ou igual que 0 , isto é,

$$
\Delta=(2 \overline{\langle L x, y\rangle}\langle L x, y\rangle)^{2}-4 \overline{\langle L x, y\rangle}\langle L x, y\rangle\langle L y, y\rangle\langle L x, x\rangle \leq 0 .
$$

Dado que $\langle L x, y\rangle \neq 0, \overline{\langle L x, y\rangle}\langle L x, y\rangle>0$. Então, $\overline{\langle L x, y\rangle}\langle L x, y\rangle-\langle L y, y\rangle\langle L x, x\rangle \leq 0$, isto é,

$$
\overline{\langle L x, y\rangle}\langle L x, y\rangle \leq\langle L y, y\rangle\langle L x, x\rangle .
$$

É claro que a desigualdade anterior também vale quando $\langle L x, y\rangle=0$, pois $L$ é definido positivo. Portanto, a desigualdade (3.4.3) vale para todo $x, y \in H$. Daí, se $\|x\|=1$ e $y=L x$, temos

$$
\|L x\|^{4} \leq\left\langle L^{2} x, L x\right\rangle\langle L x, x\rangle \leq\left\|L^{2} x\right\|\|L x\|\langle L x, x\rangle \leq\|L\|\|L x\|^{2}\langle L x, x\rangle,
$$

isto é,

$$
\|L x\|^{2} \leq\|L\|\langle L x, x\rangle \quad \text { para todo } x \in H \operatorname{com}\|x\|=1
$$


Dado que $L$ é um isomorfismo, existe $l_{1}>0$ tal que

$$
l_{1} \leq \inf _{\|x\|=1}\|L x\|^{2} .
$$

Tomando $l=l_{1} /\|L\|$, de (3.4.4) temos

$$
l \leq \inf _{\|x\|=1}\langle L x, x\rangle,
$$

o que prova o lema.

Observação 3.4.6. Seja $L \in L(H)$ un isomorfismo definido negativo em um espaço de Hilbert real ou complexo $H$. Do lema anterior se segue que existe $l>0$ tal que

$$
l \leq \inf _{\|x\|=1}\langle-L x, x\rangle,
$$

pois $-L$ é um isomorfismo definido positivo.

Observe que

$$
\inf _{\|x\|=1}\langle-L x, x\rangle=-\sup _{\|x\|=1}\langle L x, x\rangle .
$$

Portanto, existe $l^{\prime}=-l<0$ tal que

$$
l^{\prime} \geq \sup _{\|x\|=1}\langle L x, x\rangle
$$

Como vimos no começo desta seção, se $H$ tem dimensão finita, então

$$
\Phi_{S}^{+}(H)=\Phi_{S}^{-}(H)=L_{S}(H) .
$$

Neste caso, é claro que $\Phi_{S}^{+}(H)$ e $\Phi_{S}^{-}(H)$ são subconjuntos convexos de $L(H)$, pois $L_{S}(H)$ é um subespaço de $L(H)$. A continuação provaremos o caso em que $H$ tem dimensão infinita.

Teorema 3.4.7. Se $H$ é um espaço de Hilbert de dimensão infinita, então $\Phi_{S}^{+}(H)$ e $\Phi_{S}^{-}(H)$ são subconjuntos convexos de $L(H)$.

Demonstração. Primeiro provemos que $\Phi_{S}^{+}(H)$ é convexo. Fixemos $L, T \in \Phi_{S}^{+}(H)$ e $t \in[0,1]$. Denotemos por $A$ o operador $t L+(1-t) T$. Então, $A$ é auto-adjunto e portanto admite os subespaços espectrais $H_{+}(A)$ e $H_{-}(A)$. O subespaço $H_{+}(L) \cap H_{+}(T)$ é fechado, pois é a interseção de dois subespaços fechados. Além disso, $H_{+}(L) \cap$ $H_{+}(T) \neq\{0\}$, já que $H_{+}(L)$ e $H_{+}(T)$ têm codimensão finita (Lema 1.2.10). Seja $u \in H_{+}(L) \cap H_{+}(T) \operatorname{com} u \neq 0$. Assim,

$$
\langle A u, u\rangle=\langle t L u+(1-t) T u, u\rangle=t\langle L u, u\rangle+(1-t)\langle T u, u\rangle>0,
$$


isto é, $A$ é positivo em $H_{+}(L) \cap H_{+}(T)$. Logo,

$$
H_{-}(A) \cap\left(H_{+}(L) \cap H_{+}(T)\right)=\{0\} .
$$

Isto prova que a soma

$$
H_{-}(A) \oplus\left(H_{+}(L) \cap H_{+}(T)\right)
$$

é de fato direta.

A codimensão de $H_{+}(L) \cap H_{+}(T)$ em $H$ é finita pelo Lema 1.2.10. Portanto, de (3.4.5) temos

$$
\operatorname{dim} H_{-}(A)<\infty .
$$

Por último provemos que $A$ é de Fredholm. O operador $A$ é injetor na interseção $H_{+}(L) \cap H_{+}(T)$, pois $A$ é positivo em $H_{+}(L) \cap H_{+}(T)$. Portanto, dado que $H_{+}(L) \cap$ $H_{+}(T)$ tem codimensão finita,

$$
\operatorname{dim} \operatorname{Ker} A<\infty \text {. }
$$

Agora mostremos que $\operatorname{Im} A$ tem codimensão finita. Para este fim, sabendo que $A$ é auto-adjunto e que seu núcleo tem dimensão finita, pela Proposição 3.4.1 é suficiente provar que a imagem de $A$ é fechada. Dado que a restrição $L_{+}: H_{+}(L) \rightarrow H_{+}(L)$ do operador $L$ é um isomorfismo definido positivo, do lema anterior temos que existe $l_{1}>0$ tal que

$$
l_{1} \leq \inf _{\substack{\|x\|=1 \\ x \in H_{+}(L)}}\left\langle L_{+} x, x\right\rangle
$$

Daí,

$$
l_{1} \leq \inf _{\substack{\|x\|=1 \\ x \in H_{+}(L)}}\left\langle L_{+} x, x\right\rangle \leq \inf _{\substack{\|x\|=1 \\ x \in H_{+}(L) \cap H_{+}(T)}}\langle L x, x\rangle .
$$

Analogamente, existe $l_{2}>0$ tal que

$$
l_{2} \leq \inf _{\substack{\|x\|=1 \\ x \in H_{+}(L) \cap H_{+}(T)}}\langle T x, x\rangle .
$$

Portanto, se $x \in H_{+}(L) \cap H_{+}(T)$ com $\|x\|=1$,

$$
\|A x\| \geq\langle A x, x\rangle=t\langle L x, x\rangle+(1-t)\langle T x, x\rangle \geq t l_{1}+(1-t) l_{2}>0 .
$$

Logo, da Proposição 1.1.10 se segue que a restrição

$$
A_{1}=\left.A\right|_{H_{+}(L) \cap H_{+}(T)}: H_{+}(L) \cap H_{+}(T) \rightarrow A\left(H_{+}(L) \cap H_{+}(T)\right)
$$

é inversível. Assim,

$$
\operatorname{Im} A_{1}=A\left(H_{+}(L) \cap H_{+}(T)\right)
$$


é fechada. Como $H_{+}(L) \cap H_{+}(T)$ tem codimensão finita em $H$, existe um subespaço de dimensão finita $H_{2}$ de $H$ tal que

$$
\operatorname{Im} A=\operatorname{Im} A_{1} \oplus H_{2} .
$$

Logo, $\operatorname{Im} A=\operatorname{Im}(t L+(1-t) T)$ é fechada, pois é a soma de um espaço fechado e um espaço de dimensão finita (Lema 1.2.3). Consequentemente, $t L+(1-t) T$ é de Fredholm. Em conclusão, $t L+(1-t) T \in \Phi_{S}^{+}(H)$.

Por outro lado, é claro que um operador $M$ pertence a $\Phi_{S}^{+}(H)$ se, e somente se, $-M$ pertence a $\Phi_{S}^{-}(H)$. Portanto, se $L, T \in \Phi_{S}^{-}(H)$, então $-L,-T \in \Phi_{S}^{+}(H)$. Assim, pela primeira parte da prova, $t(-L)+(1-t)(-T) \in \Phi_{S}^{+}(H)$, isto é, $t L+(1-t) T \in \Phi_{S}^{-}(H)$.

Denotaremos por $G L_{S}^{+}(H)$ o subconjunto de $L(H)$ dos isomorfismos definidos positivos.

Suponhamos que $H$ seja de dimensão finita. Sejam $L, T \in G L_{S}^{+}(H)$ e $t \in[0,1]$. É fácil ver que $t L+(1-t) T$ é definido positivo. Assim, $t L+(1-t) T$ é injetor. Como $H$ tem dimensão finita, $t L+(1-t) T$ é um isomorfismo. Este fato mostra que $G L_{S}^{+}(H)$ é um subconjunto convexo de $L(H)$.

Como consequência do teorema anterior, o seguinte corolário mostra o caso em que $H$ tem dimensão infinita.

Corolário 3.4.8. Se $H$ tem dimensão infinita, o conjunto $G L_{S}^{+}(H)$ é convexo em $L(H)$.

Demonstração. De fato, sejam $L$ e $T$ isomorfismos definidos positivos e $t \in[0,1]$ fixados. Observe que $t L+(1-t) T$ é definido positivo. Assim,

$$
\operatorname{Ker}(t L+(1-t) T)=\{0\} .
$$

Além disso, do teorema anterior temos que $t L+(1-t) T$ é de Fredholm. Dado que $t L+(1-t) T$ é auto-adjunto, a Observação 3.4.2 implica que

$$
\operatorname{Im}(t L+(1-t) T)=H .
$$

Consequentemente, $t L+(1-t) T$ é um isomorfismo definido positivo.

Analogamente podemos provar que o conjunto dos isomorfismos definidos negativos é convexo em $L(H)$.

Na Seção 3.2 provamos que $L_{S}(H)$ é um subespaço fechado de $L(H)$. Consideremos $L_{S}(H)$ como subespaço topológico de $L(H)$, isto é, com a topologia herdada de $L(H)$. Uma outra propriedade que possui o conjunto $G L_{S}^{+}(H)$ é apresentada na seguinte proposição.

Proposição 3.4.9. $O$ conjunto $G L_{S}^{+}(H)$ é aberto em $L_{S}(H)$. 
Demonstração. Seja $L \in G L_{S}^{+}(H)$ fixado. Se segue do Lema 3.4.5 que existe $l>0$ tal que

$$
l \leq \inf _{\|x\|=1}\langle L x, x\rangle .
$$

Seja $T$ um operador auto-adjunto tal que $\|L-T\|<\min \left\{1 /\left\|L^{-1}\right\|, l\right\}$. Então, $T$ é um isomorfismo (veja-se o Corolário 1.1.8) e, além disso, para $x \in H \operatorname{com} x \neq 0$, temos

$$
\langle L x, x\rangle \geq l\langle x, x\rangle>\|L-T\|\langle x, x\rangle=\langle\|L-T\| x, x\rangle \geq\langle(L-T) x, x\rangle,
$$

isto é,

$$
0<\langle L x, x\rangle-\langle(L-T) x, x\rangle=\langle T x, x\rangle \quad \text { para todo } x \in H \operatorname{com} x \neq 0
$$

Assim, $T$ é um isomorfismo definido positivo, o que prova a proposição.

Agora provaremos que, se $H$ é um espaço de Hilbert real, de dimensão infinita e separável, o conjunto dos operadores de Fredholm fortemente indefinidos em $L(H)$ é conexo por caminhos. Para este fim, vejamos primeiro os seguintes resultados.

Suponhamos que $L \in \Phi_{S}(H)$ e $S \in G L(H)$, onde $H$ é um espaço de Hilbert real (de dimensão finita ou infinita) não necessariamente separável. Observe que o operador $\widetilde{L}=S^{*} L S$ é um operador de Fredholm auto-adjunto. De fato, $\widetilde{L}$ é de Fredholm, pois a composição de operadores de Fredholm é um operador de Fredholm. Além disso,

$$
\widetilde{L}^{*}=\left(S^{*} L S\right)^{*}=S^{*} L^{*} S=S^{*} L S=\widetilde{L},
$$

isto é, $\widetilde{L}$ é auto-adjunto.

Definição 3.4.10 (Ação cogradiente). A ação cogradiente é a aplicação $\Upsilon$ : $G L(H) \times$ $\Phi_{S}(H) \rightarrow \Phi_{S}(H)$ definida por

$$
\Upsilon(S, L)=S^{*} L S
$$

No resto desta seção suporemos que $H$ seja um espaço de Hilbert real de dimensão infinita.

Proposição 3.4.11. Os espaços $\Phi_{S}^{+}(H), \Phi_{S}^{-}(H)$ e $\Phi_{S}^{i}(H)$ são invariantes pela ação cogradiente, isto é, para qualquer $S \in G L(H)$ fixado, temos que:

i. se $L \in \Phi_{S}^{+}(H)$, então $S^{*} L S \in \Phi_{S}^{+}(H)$;

ii. se $L \in \Phi_{S}^{-}(H)$, então $S^{*} L S \in \Phi_{S}^{-}(H)$;

iii. se $L \in \Phi_{S}^{i}(H)$, então $S^{*} L S \in \Phi_{S}^{i}(H)$. 
Demonstração. Sejam $L \in \Phi_{S}(H)$ e $S \in G L(H)$ fixados. Ponhamos $\widetilde{L}=S^{*} L S$. Podemos expressar o espaço $H$ como

$$
H=H_{+}(L) \oplus H_{-}(L) \oplus \operatorname{Ker} L \quad \text { e } \quad H=H_{+}(\widetilde{L}) \oplus H_{-}(\widetilde{L}) \oplus \operatorname{Ker} \widetilde{L} .
$$

Para provar o caso $i$. suponhamos que $L \in \Phi_{S}^{+}(H)$. O operador $\widetilde{L}$ é positivo em $S^{-1}\left(H_{+}(L)\right)$, pois, se $x \in H_{+}(L) \operatorname{com} x \neq 0$, então

$$
\left\langle\widetilde{L} S^{-1} x, S^{-1} x\right\rangle=\left\langle S^{*} L S S^{-1} x, S^{-1} x\right\rangle=\left\langle S^{*} L x, S^{-1} x\right\rangle=\left\langle L x, S S^{-1} x\right\rangle=\langle L x, x\rangle>0 .
$$

Logo, a soma

$$
S^{-1}\left(H_{+}(L)\right) \oplus H_{-}(\widetilde{L})
$$

é de fato direta. Dado que $S$ é um isomorfismo e a codimensão de $H_{+}(L)$ é finita, pois $L \in \Phi_{S}^{+}(H)$, então a codimensão de $S^{-1}\left(H_{+}(L)\right)$ em $H$ é finita. Portanto, sendo direta a soma em (3.4.6), obtemos que $\operatorname{dim} H_{-}(\widetilde{L})<\infty$. Daí, $\widetilde{L} \in \Phi_{S}^{+}(H)$, o que prova $i$.

Agora, para o caso ii. suponhamos que $L \in \Phi_{S}^{-}(H)$. Logo, $-L \in \Phi_{S}^{+}(H)$. Pelo fato anterior temos que $S^{*}(-L) S \in \Phi_{S}^{+}(H)$. Assim, $S^{*} L S \in \Phi_{S}^{-}(H)$.

Por último, suponhamos que $L \in \Phi_{S}^{i}(H)$. Já que $\widetilde{L}$ é de Fredholm, $\operatorname{dim} \operatorname{Ker} \widetilde{L}<\infty$.

Dado que a soma

$$
S^{-1}\left(H_{+}(L)\right) \oplus H_{-}(\widetilde{L})
$$

é direta e a dimensão de $S^{-1}\left(H_{+}(L)\right)$ é infinita, temos que a codimensão de $H_{-}(\widetilde{L})$ é infinita. Portanto, como $\operatorname{dim} \operatorname{Ker} \widetilde{L}<\infty$, então

$$
\operatorname{dim} H_{+}(\widetilde{L})=\infty
$$

Analogamente,

$$
\operatorname{dim} H_{-}(\widetilde{L})=\infty
$$

Logo, $\widetilde{L} \in \Phi_{S}^{i}(H)$, o que prova o caso $i i i$.

A continuação apresentaremos alguns outros resultados que também serão muito úteis para a construção do fluxo espectral.

Proposição 3.4.12. Se $L \in \Phi_{S}^{i}(H)$ e $K$ é um operador auto-adjunto com imagem de dimensão finita, então $L+K \in \Phi_{S}^{i}(H)$. 
Demonstração. O operador $L+K$ é de Fredholm, pois $L$ é de Fredholm e $K$ é compacto. Logo, como $L$ e $K$ são auto-adjuntos, $L+K \in \Phi_{S}(H)$.

Dado que $\operatorname{Im} K$ é fechada (sendo finito-dimensional) e $K$ é auto-adjunto, de (3.2.5) temos que

$$
H=\operatorname{Im} K \oplus \operatorname{Ker} K .
$$

Daí, Ker $K$ tem codimensão finita. Consequentemente, o Lema 1.2.11 implica que Ker $K \cap H_{+}(L)$ e Ker $K \cap H_{-}(L)$ têm dimensão infinita, pois $H_{+}(L)$ e $H_{-}(L)$ têm dimensão infinita. Assim, já que $L$ e $L+K$ coincidem em Ker $K$, se segue que $L+K$ é positivo em Ker $K \cap H_{+}(L)$ e negativo em $\operatorname{Ker} K \cap H_{-}(L)$. Este fato implica que $H_{+}(L+K)$ e $H_{-}(L+K)$ têm codimensão infinita. Portanto,

$$
\operatorname{dim} H_{+}(L+K)=\infty \quad \text { e } \quad \operatorname{dim} H_{-}(L+K)=\infty .
$$

Em conclusão, $L+K \in \Phi_{S}^{i}(H)$.

Definição 3.4.13 (Espaços contráteis). Um espaço topológico $\Lambda$ é contrátil se existe uma homotopia

$$
h: \Lambda \times[0,1] \rightarrow \Lambda,
$$

tal que

$$
h(\lambda, 0)=\lambda \quad \text { e } \quad h(\lambda, 1)=\lambda_{0} \quad \text { para todo } \lambda \in \Lambda,
$$

onde $\lambda_{0} \in \Lambda$ é fixado.

O teorema seguinte é consequência de um resultado devido a Kuiper (veja-se [17], Teorema 2) que diz que, se $H$ é separável, o conjunto $G L(H)$ é contrátil.

Teorema 3.4.14. Se $H$ é separável, $G L(H)$ é conexo por caminhos.

Demonstração. Consideremos uma homotopia

$$
h: H \times[0,1] \rightarrow H,
$$

tal que

$$
h(x, 0)=x \quad \text { e } \quad h(x, 1)=x_{0} \quad \text { para todo } x \in H,
$$

onde $x_{0} \in H$ é fixado. Para $x \in H$, tomamos a aplicação $f(t)=h(x, t)$ para $t \in$ $[0,1]$. É claro que $f$ é uma aplicação contínua que liga ao ponto $x$ com o ponto $x_{0}$. Consequentemente, $H$ é conexo por caminhos.

Agora podemos anunciar e provar a conexidade do conjunto $\Phi_{S}^{i}(H)$.

Teorema 3.4.15. Se H é um espaço de Hilbert real, de dimensão infinita e separável, o conjunto $\Phi_{S}^{i}(H)$ é conexo por caminhos. 
Demonstração. Sejam $H_{+}$e $H_{-}$dois subespaços fechados de $H$, ortogonais, de dimensão infinita e tais que $H=H_{+} \oplus H_{-}$. Fixemos o operador $\mathcal{J} \in L(H)$ que tem como matriz de operadores

$$
\mathcal{J}=\left(\begin{array}{cc}
\left.I\right|_{H_{+}} & 0 \\
0 & -\left.I\right|_{H_{-}}
\end{array}\right)
$$

onde $\left.I\right|_{H_{+}}$e $\left.I\right|_{H_{-}}$denotam a identidade de $H_{+}$e $H_{-}$, respectivamente. É claro que $\mathcal{J}$ é um isomorfismo auto-adjunto. Além disso,

$$
H_{+}(\mathcal{J})=H_{+} \quad \text { e } \quad H_{-}(\mathcal{J})=H_{-},
$$

isto é, $\mathcal{J} \in \Phi_{S}^{i}(H)$.

Se provarmos que qualquer operador $L \in \Phi_{S}^{i}(H)$ pode ser ligado com $\mathcal{J}$ por um caminho contínuo, então teremos provado o teorema. De fato, seja $L \in \Phi_{S}^{i}(H)$ fixado. Vejamos que, para todo $t \in[0,1]$, o operador $\mathcal{I}_{t}: H \rightarrow H$ definido por

$\mathcal{I}_{t}=(1-t) P_{H_{+}(L)} L P_{H_{+}(L)}+t P_{H_{+}(L)} I P_{H_{+}(L)}+(1-t) P_{H_{-}(L)} L P_{H_{-}(L)}-t P_{H_{-}(L)} I P_{H_{-}(L)}$

pertence a $\Phi_{S}^{i}(H)$, onde $P_{H_{+}(L)}$ e $P_{H_{-}(L)}$ denotam as projeções ortogonais sobre os espaços $H_{+}(L)$ e $H_{-}(L)$, respectivamente. Dado que as restrições dos operadores $L \mathrm{e}$ $I$ ao espaço $H_{+}(L)$ são isomorfismos definidos positivos e o conjunto dos isomorfismos definidos positivos é convexo (Corolário 3.4.8), temos que a restrição

$$
\left.\mathcal{I}_{t}\right|_{H_{+}(L)}=(1-t) P_{H_{+}(L)} L P_{H_{+}(L)}+t P_{H_{+}(L)} I P_{H_{+}(L)}: H_{+}(L) \rightarrow H_{+}(L)
$$

é um isomorfismo definido positivo para todo $t \in[0,1]$. Analogamente,

$$
\left.\mathcal{I}_{t}\right|_{H_{-}(L)}=(1-t) P_{H_{-}(L)} L P_{H_{-}(L)}+t P_{H_{-}(L)}(-I) P_{H_{-}(L)}: H_{-}(L) \rightarrow H_{-}(L)
$$

é um isomorfismo definido negativo para todo $t \in[0,1]$. Observe que, para $t \in[0,1]$, o operador $\mathcal{I}_{t}$ é auto-adjunto, pois $L, P_{H_{+}(L)}, P_{H_{-}(L)}$ e $I$ são auto-adjuntos. Além disso, $\operatorname{Ker} \mathcal{I}_{t}=\operatorname{Ker} L$ para todo $t \in[0,1]$. Assim, para $t \in[0,1], \mathcal{I}_{t}$ é um operador de Fredholm fortemente indefinido. Além disso, pelas fórmulas (3.4.7) e (3.4.8) e as propriedades acima de $\mathcal{I}_{t}$, temos que

$$
H_{+}\left(\mathcal{I}_{t}\right)=H_{+}(L), \quad H_{-}\left(\mathcal{I}_{t}\right)=H_{-}(L) \quad \text { e } \quad \operatorname{Ker} \mathcal{I}_{t}=\operatorname{Ker} L .
$$

Daí, para $t \in[0,1], \mathcal{I}_{t}$ define um caminho em $\Phi_{S}^{i}(H)$ tal que

$$
\mathcal{I}_{0}=L \quad \text { e } \quad \mathcal{I}_{1}=\mathcal{I}
$$

onde $\mathcal{I}$ é o operador em $\Phi_{S}^{i}(H)$ definido como

$$
\left.\mathcal{I}\right|_{H_{+}(L)}=\left.I\right|_{H_{+}(L)},\left.\quad \mathcal{I}\right|_{H_{-}(L)}=-\left.I\right|_{H_{-}(L)} \quad \text { e }\left.\quad \mathcal{I}\right|_{\text {Ker } L}=\left.0\right|_{\text {Ker } L},
$$


onde $\left.0\right|_{\text {Ker } L}$ é o operador nulo no núcleo de $L$.

Agora, da Proposição 3.4.12 se segue que $\alpha(t)=\mathcal{I}+t P_{\operatorname{Ker} L} \in \Phi_{S}^{i}(H)$ para todo $t \in[0,1]$, onde $P_{\text {Ker } L}$ é a projeção ortogonal sobre Ker $L$. Logo, $\alpha:[0,1] \rightarrow \Phi_{S}^{i}(H)$ é um caminho tal que

$$
\alpha(0)=\mathcal{I} \quad \text { e } \quad \alpha(1)=\mathcal{I}+P_{\operatorname{Ker} L} .
$$

Por outro lado, do Teorema 1.1 .18 se segue que $H_{+}(L) \oplus \operatorname{Ker} L$ é isométrico a $H_{+}$ e $H_{-}(L)$ é isométrico a $H_{-}$, isto é, existem dois operadores ortogonais

$$
M_{+}: H_{+}(L) \oplus \operatorname{Ker} L \rightarrow H_{+} \quad \text { e } \quad M_{-}: H_{-}(L) \rightarrow H_{-} .
$$

Tomemos o operador $M: H \rightarrow H$ dado por

$$
\left.M\right|_{H_{+}(L) \oplus \operatorname{Ker} L}=M_{+} \quad \text { e }\left.\quad M\right|_{H_{-}(L)}=M_{-} .
$$

Observe que $M^{*} \mathcal{J} M=\mathcal{I}+P_{\text {Ker } L}$. De fato, se $x \in H_{+}(L) \oplus \operatorname{Ker} L$, então $M x=$ $M_{+} x \in H_{+}$. Assim, $\mathcal{J} M_{+} x=M_{+} x$ e

$$
M^{*} \mathcal{J} M x=M_{+}^{*} \mathcal{J} M_{+} x=M_{+}^{*} M_{+} x=x=\left(\mathcal{I}+P_{\text {Ker } L}\right) x .
$$

Agora, se $x \in H_{-}(L)$, então $\mathcal{J} M x=-M_{-} x \in H_{-}$. Logo,

$$
M^{*} \mathcal{J} M x=-M_{-}^{*} M_{-} x=-x=\mathcal{I} x=\left(\mathcal{I}+P_{\text {Ker } L}\right) x .
$$

De (3.4.11) e (3.4.12) obtemos

$$
M^{*} \mathcal{J} M=\mathcal{I}+P_{\text {Ker } L}
$$

O Teorema 3.4.14 prova que existe um caminho $M:[0,1] \rightarrow G L(H)$ tal que $M(0)=M$ e $M(1)=I$. Se segue da Proposição 3.4.11 que

$$
\mathcal{J}_{t}=M(t)^{*} \mathcal{J} M(t) \in \Phi_{S}^{i}(H) \text { para todo } t \in[0,1] .
$$

Logo, para $t \in[0,1], \mathcal{J}_{t}$ define um caminho em $\Phi_{S}^{i}(H)$ tal que

$$
\mathcal{J}_{0}=M^{*} \mathcal{J} M=\mathcal{I}+P_{\text {Ker } L} \quad \text { e } \quad \mathcal{J}_{1}=\mathcal{J} .
$$

De (3.4.9), (3.4.10) e (3.4.13) se segue que, para qualquer $L \in \Phi_{S}^{i}(H)$, existe um caminho $\beta:[0,1] \rightarrow \Phi_{S}^{i}(H)$ tal que $\beta(0)=L$ e $\beta(1)=\mathcal{J}$. Em conclusão, $\Phi_{S}^{i}(H)$ é conexo por caminhos.

Como falamos na introdução desta seção, mostraremos que $\Phi_{S}^{+}(H), \Phi_{S}^{-}(H)$ e $\Phi_{S}^{i}(H)$ são as três componentes conexas de $\Phi_{S}(H)$. Para este fim, dado que

$$
\Phi_{S}(H)=\Phi_{S}^{+}(H) \cup \Phi_{S}^{-}(H) \cup \Phi_{S}^{i}(H)
$$

e os espaços $\Phi_{S}^{+}(H), \Phi_{S}^{-}(H)$ e $\Phi_{S}^{i}(H)$ são conexos dois a dois disjuntos, pelos Teoremas 3.4 .7 e 3.4.15, é suficiente provar que $\Phi_{S}^{+}(H), \Phi_{S}^{-}(H)$ e $\Phi_{S}^{i}(H)$ são abertos em $\Phi_{S}(H)$. Concluímos esta seção com a prova deste fato. 
Teorema 3.4.16. Os espaços $\Phi_{S}^{+}(H), \Phi_{S}^{-}(H)$ e $\Phi_{S}^{i}(H)$ são subconjuntos abertos de $\Phi_{S}(H)$.

Demonstração. Provemos primeiro que $\Phi_{S}^{+}(H)$ é aberto em $\Phi_{S}(H)$. De fato, dado que o conjunto dos operadores de Fredholm é um subconjunto aberto de $L(H)$, temos que o conjunto dos operadores de Fredholm auto-adjuntos é um subconjunto aberto de $L_{S}(H)$, isto é, $\Phi_{S}(H)$ é aberto em $L_{S}(H)$. Tomemos $L \in \Phi_{S}^{+}(H)$ fixado. Como a restrição $L_{+}=\left.L\right|_{H_{+}}: H_{+}(L) \rightarrow H_{+}(L)$ de $L$ é um isomorfismo, se segue do Lema 3.4.5 que existe $l>0$ tal que

$$
l \leq \inf _{\substack{\|x\|=1 \\ x \in H_{+}(L)}}\langle L x, x\rangle .
$$

Já que $\Phi_{S}(H)$ é aberto em $L_{S}(H)$, existe $\varepsilon>0$, com $\varepsilon<l$, tal que

$$
B(L, \varepsilon) \cap L_{S}(H) \subseteq \Phi_{S}(H),
$$

onde $B(L, \varepsilon)$ é a bola em $L(H)$ com centro em $L$ e raio $\varepsilon$. Vejamos que $B(L, \varepsilon) \cap$ $L_{S}(H) \subseteq \Phi_{S}^{+}(H)$. De fato, se $T \in B(L, \varepsilon) \cap L_{S}(H)$, então, para $x \in H_{+}(L)$ com $\|x\|=1$

$$
\langle(L-T) x, x\rangle \leq\|L-T\|<\varepsilon
$$

Daí,

$$
l \leq\langle L x, x\rangle<\varepsilon+\langle T x, x\rangle \quad \text { para todo } x \in H_{+}(L) \operatorname{com}\|x\|=1 .
$$

Este fato prova que $0<l-\varepsilon<\langle T x, x\rangle$ para todo $x \in H_{+}(L)$ com $\|x\|=1$. Consequentemente, $T$ é positivo em um espaço de codimensão finita. Logo, a dimensão de $H_{-}(T)$ é finita, isto é, $T \in \Phi_{S}^{+}(H)$, como queríamos provar.

Por outro lado, dado que $L \in \Phi_{S}^{-}(H)$ se, e somente se, $-L \in \Phi_{S}^{+}(H)$, da prova acima temos que existe uma bola $B(-L, \varepsilon)$ com centro em $-L$ e raio $\varepsilon>0$ tal que $B(-L, \varepsilon) \cap L_{S}(H) \subseteq \Phi_{S}^{+}(H)$. Portanto, dado que

$$
-B(-L, \varepsilon)=\{-T: T \in B(-L, \varepsilon)\}=B(L, \varepsilon),
$$

temos

$$
B(L, \varepsilon) \cap L_{S}(H)=[-B(-L, \varepsilon)] \cap L_{S}(H) \subseteq \Phi_{S}^{-}(H) .
$$

Este fato prova que $\Phi_{S}^{-}(H)$ é aberto em $\Phi_{S}(H)$.

Por último, vejamos que $\Phi_{S}^{i}(H)$ é aberto em $\Phi_{S}(H)$. De fato, sejam $L \in \Phi_{S}^{i}(H)$ e $l_{1}$ e $l_{2}$ tais que

$$
0<l_{1} \leq \inf _{\substack{\|x\|=1 \\ x \in H_{+}(L)}}\langle L x, x\rangle \quad \text { e } \quad 0<l_{2} \leq \inf _{\substack{\|x\|=1 \\ x \in H_{-}(L)}}\langle-L x, x\rangle .
$$


Como acima, existe $\varepsilon>0, \operatorname{com} \varepsilon<\min \left\{l_{1}, l_{2}\right\}$, tal que

$$
B(L, \varepsilon) \cap L_{S}(H) \subseteq \Phi_{S}(H)
$$

Tomemos $T \in B(L, \varepsilon) \cap L_{S}(H)$ fixado. De forma análoga ao fato obtido em (3.4.14), obtemos que $T$ é positivo em $H_{+}(L)$ e $-T$ é positivo em $H_{-}(L)$. Logo, $T$ é positivo em $H_{+}(L)$ e é negativo em $H_{-}(L)$. Consequentemente, $H_{+}(T)$ e $H_{-}(T)$ têm dimensão infinita. Portanto, $T \in \Phi_{S}^{i}(H)$ e $\operatorname{assim} B(L, \varepsilon) \cap L_{S}(H) \subseteq \Phi_{S}^{i}(H)$. Em conclusão, $\Phi_{S}^{i}(H)$ é aberto em $\Phi_{S}(H)$. 


\section{Capítulo 4}

\section{A assinatura generalizada e o índice de Morse relativo}

Em dimensão finita, os operadores auto-adjuntos permitem a representação de um espaço de Hilbert $H$ como soma direta ortogonal dos subespaços espectrais positivo e negativo e do núcleo do operador (Teorema espectral em dimensão finita). Neste caso a assinatura de um isomorfismo auto-adjunto é definida como a diferença entre a dimensão do seu subespaço espectral positivo e a dimensão do seu subespaço espectral negativo. Neste capítulo generalizaremos esta definicão de assinatura para perturbações compactas auto-adjuntas de um particular operador de Fredholm autoadjunto fortemente indefinido, cujo quadrado é a identidade e que chamaremos de simetria. A continuidade da aplicação assinatura, quando considerada no conjunto dos isomorfismos auto-adjuntos em um espaço de Hilbert de dimensão finita, que provaremos na seguinte seção, é crucial para a existência da assinatura generalizada, ou seja, em dimensão infinita. O fluxo espectral será definido fazendo uso da assinatura generalizada e da ação cogradiente, definida no capítulo anterior.

Na próxima seção veremos outras propriedades que possui a assinatura em dimensão finita. Uma destas propriedades é a invariância pela ação cogradiente. Além disso, mostraremos que, dado um isomorfismo auto-adjunto $L \in L(H)$, se expressarmos $H$ como a soma de dos subespaços ortogonais $H_{1}$ e $H_{2}$ invariantes por $L$, então a assinatura de $L$ é igual à soma das assinaturas das restrições do operador $L$ a $H_{1}$ e $H_{2}$.

Na segunda seção apresentaremos a definicão da assinatura generalizada. Veremos que esta definição não é invariante pela ação cogradiente. Porém, o fluxo espectral, que será definido no próximo capítulo fazendo uso da assinatura generalizada, é invariante por esta ação.

O propósito da terceira seção é provar que aplicação que associa a um operador auto-adjunto $L$ a projeção ortogonal $P_{H_{-}(L)}$ sobre seu subespaço espectral negativo (positivo) é contínua. Para este fim, com base no Teorema integral de Cauchy para aplicações complexas, veremos primeiro que, se $E$ é um espaço de Banach complexo, 
$L \in L(E)$ e $f: \Delta \rightarrow \mathbb{C}$ é uma aplicação regular, onde $\Delta$ é um subconjunto aberto de $\mathbb{C}$ que contém o espectro de $L, f(L)$ é bem definido como um operador em $L(E)$. Fazendo uso do fato anterior, provaremos que, se $H$ é um espaço de Hilbert complexo e $L \in L(H)$ é auto-adjunto e 0 não é ponto de acumulação de $\sigma(L)$, a projeção ortogonal sobre o subespaço espectral negativo de $L$ pode ser expressada como um operador $\chi(L)$, onde $\chi: \Delta \rightarrow \mathbb{C}$ é uma oportuna aplicação regular tal que $\sigma(L) \subseteq \Delta$. De forma análoga podemos representar a projeção ortogonal sobre o subespaço espectral positivo de $L$. Esta nova expressão para tais projeções permitirá mostrar que elas dependem continuamente do operador, fato que será fundamental no resto do trabalho.

Na quarta seção definiremos o índice de Morse relativo para pares de isomorfismos auto-adjuntos em espaços de Hilbert. No último capítulo provaremos que o fluxo espectral de um caminho de perturbações compactas auto-adjuntas de um operador de Fredholm fixado é igual ao índice de Morse relativo do par formado pelos extremos do caminho.

\subsection{A assinatura em espaços de Hilbert de dimen- são finita}

Como foi dito na introdução deste capítulo, nesta seção veremos a definição e algumas das propriedades que possui a aplicação assinatura sign : $G L_{S}(H) \rightarrow \mathbb{R}$, onde $G L_{S}(H)$ denota o conjunto dos isomorfismos auto-adjuntos em um espaço de Hilbert de dimensão finita $H$. Uma destas propriedades é a continuidade, que será provada no final desta seção.

A assinatura generalizada, que será definida na próxima seção, não é invariante pela ação cogradiente. Porém, mostraremos que a assinatura de um operador inversível auto-adjunto em um espaço de Hilbert de dimensão finita é invariante por esta ação. As propriedades da assinatura, que apresentaremos nesta seção, serão fundamentais na definição da assinatura generalizada.

Nesta seção vamos supor que $H$ seja um espaço de Hilbert real de dimensão finita. Para um operador auto-adjunto $L \in L(H)$, denotaremos por $\mu(L)$ a dimensão do subespaço espectral negativo de $L$. Este número é também conhecido como índice de Morse do operador.

Definição 4.1.1. A assinatura de um isomorfismo auto-adjunto $L \in L(H)$ é definida por

$$
\operatorname{sign} L=\mu(-L)-\mu(L) .
$$

A definição acima quer dizer que a assinatura de um isomorfismo auto-adjunto em um espaço de Hilbert de dimensão finita é igual à diferença entre as dimensões dos seus subespaços espectrais positivo e negativo, isto é,

$$
\operatorname{sign} L=\operatorname{dim} H_{+}(L)-\operatorname{dim} H_{-}(L) .
$$


Lembremos que, se $L \in L(H)$ é auto-adjunto, então $H_{+}(L)$ coincide com o autoespaço gerado pelos autovetores de $L$ relativos aos autovalores positivos e $H_{-}(L)$ coincide com o autoespaço gerado pelos autovetores relativos aos autovalores negativos.

Observação 4.1.2. Seja $L \in L(H)$ auto-adjunto. Observe que, como

$$
H_{+}(L)=\operatorname{span}\{v: v \text { é um autovetor de } L \text { com autovalor positivo }\}
$$

$\mathrm{e}$

$$
H_{-}(L)=\operatorname{span}\{v: v \text { é um autovetor de } L \text { com autovalor negativo }\},
$$

a dimensão de $H_{+}(L)$ é igual ao número de autovalores positivos de $L$ (contando as multiplicidades) e a dimensão de $H_{-}(L)$ é igual ao número de autovalores negativos de $L$ (contando as multiplicidades). Estas igualdades se obtêm pelo fato de que, se $L$ é auto-adjunto em um espaço de Hilbert de dimensão finita, a multiplicidade algébrica de cada autovalor $\lambda$ de $L$, isto é, o número de vezes que $\lambda$ se repete como raiz do polinômio característico de $L$, coincide com sua multiplicidade geométrica ${ }^{1}$, ou seja, a dimensão do autoespaço gerado pelos autovetores correspondentes a $\lambda$. Portanto, a assinatura de $L$ é igual à diferença entre o número de autovalores positivos de $L$ (contando as multiplicidades) e número de autovalores negativos de $L$ (contando as multiplicidades).

Como falamos na introdução desta secão, a assinatura é invariante pela ação cogradiente. Este fato é mostrado na seguinte proposição.

Proposição 4.1.3. Se $L: H \rightarrow H$ é um isomorfismo auto-adjunto e $S \in G L(H)$, então

$$
\operatorname{sign} S^{*} L S=\operatorname{sign} L
$$

Demonstração. É suficiente provar que

$$
\operatorname{dim} H_{+}\left(S^{*} L S\right)=\operatorname{dim} H_{+}(L) \quad \text { e } \quad \operatorname{dim} H_{-}\left(S^{*} L S\right)=\operatorname{dim} H_{-}(L) .
$$

Denotemos por $\widetilde{L}$ o operador $S^{*} L S$. Já que $L$ e $\widetilde{L}$ são isomorfismos auto-adjuntos, temos

$$
H=H_{+}(L) \oplus H_{-}(L)=H_{+}(\widetilde{L}) \oplus H_{-}(\widetilde{L}) .
$$

Em (3.4.6) vimos que a soma $S^{-1}\left(H_{+}(L)\right) \oplus H_{-}(\widetilde{L})$ é de fato direta. Agora, dado que $\operatorname{dim} S^{-1}\left(H_{+}(L)\right)=\operatorname{dim} H_{+}(L)$

$$
\operatorname{dim} H_{-}(\widetilde{L}) \leq \operatorname{dim} H_{-}(L) .
$$

\footnotetext{
${ }^{1}$ Sabemos que, se $L$ não for auto-adjunto, a multiplicidade geométrica do autovalor pode ser estritamente menor que sua multiplicidade algébrica.
} 
Analogamente,

$$
\operatorname{dim} H_{+}(\widetilde{L}) \leq \operatorname{dim} H_{+}(L) .
$$

De (4.1.1), (4.1.2) e (4.1.3) temos

$$
\operatorname{dim} H_{+}(\widetilde{L})=\operatorname{dim} H_{+}(L) \quad \text { e } \quad \operatorname{dim} H_{-}(\widetilde{L})=\operatorname{dim} H_{-}(L),
$$

o que prova o proposição.

Sejam $L \in G L(H)$ um operador auto-adjunto e $\widetilde{H}$ um espaço de Hilbert com $\operatorname{dim} \widetilde{H}=\operatorname{dim} H<\infty$. Observe que, se $O \in L(\widetilde{H}, H)$, então $O^{*} L O$ é auto-adjunto em $L(\widetilde{H})$, pois

$$
\left(O^{*} L O\right)^{*}=O^{*} L^{*} O=O^{*} L O .
$$

O seguinte lema é consequência imediata da prova da proposição anterior.

Lema 4.1.4. Sejam $H$ e $\widetilde{H}$ espaços de Hilbert com $\operatorname{dim} H=\operatorname{dim} \widetilde{H}<\infty$. Se $L \in$ $G L(H)$ é auto-adjunto e $O \in L(\widetilde{H}, H)$ é um isomorfismo, então $O^{*} L O$ é um isomorfismo auto-adjunto em $L(\widetilde{H})$ e, além disso,

$$
\operatorname{sign}\left(O^{*} L O\right)=\operatorname{sign} L .
$$

Demonstração. Acima provamos que o operador $O^{*} L O$ é auto-adjunto em $L(\widetilde{H})$. Observe que $O^{*} L O$ é um isomorfismo, sendo composição de isomofismos. De forma análoga à prova da proposição anterior podemos mostrar que

$$
\operatorname{dim} H_{+}\left(O^{*} L O\right)=\operatorname{dim} H_{+}(L) \text { e } \operatorname{dim} H_{-}\left(O^{*} L O\right)=\operatorname{dim} H_{-}(L) .
$$

Consequentemente, $\operatorname{sign}\left(O^{*} L O\right)=\operatorname{sign} L$.

Lema 4.1.5. Seja $L \in L(H)$ um isomorfismo auto-adjunto. Suponhamos que $H=$ $H_{1} \oplus H_{2}$, onde $H_{1}$ e $H_{2}$ são subespaços ortogonais de $H$, e que a matriz de operadores associada a $L$ e a esta soma seja da forma

$$
L=\left(\begin{array}{cc}
L_{1} & 0 \\
0 & L_{2}
\end{array}\right)
$$

Então,

$$
\operatorname{sign} L=\operatorname{sign} L_{1}+\operatorname{sign} L_{2} .
$$

Demonstração. É claro que $L_{1}: H_{1} \rightarrow H_{1}$ e $L_{2}: H_{2} \rightarrow H_{2}$ são isomorfismos autoadjuntos, portanto admitem as decomposições

$$
H_{1}=H_{+}\left(L_{1}\right) \oplus H_{-}\left(L_{1}\right) \quad \text { e } \quad H_{2}=H_{+}\left(L_{2}\right) \oplus H_{-}\left(L_{2}\right) .
$$


Assim,

$$
H=H_{+}\left(L_{1}\right) \oplus H_{-}\left(L_{1}\right) \oplus H_{+}\left(L_{2}\right) \oplus H_{-}\left(L_{2}\right) .
$$

É fácil ver que $H_{+}\left(L_{1}\right) \oplus H_{+}\left(L_{2}\right) \subseteq H_{+}(L)$ e $H_{-}\left(L_{1}\right) \oplus H_{-}\left(L_{2}\right) \subseteq H_{-}(L)$. Consequentemente, já que $H=H_{+}(L) \oplus H_{-}(L)$, obtemos que

$$
H_{+}(L)=H_{+}\left(L_{1}\right) \oplus H_{+}\left(L_{2}\right) \quad \text { e } \quad H_{-}(L)=H_{-}\left(L_{1}\right) \oplus H_{-}\left(L_{2}\right) .
$$

Daí,

$$
\begin{aligned}
\operatorname{sign} L & =\operatorname{dim} H_{+}(L)-\operatorname{dim} H_{-}(L) \\
& =\operatorname{dim} H_{+}\left(L_{1}\right)+\operatorname{dim} H_{+}\left(L_{2}\right)-\operatorname{dim} H_{-}\left(L_{1}\right)-\operatorname{dim} H_{-}\left(L_{2}\right) \\
& =\operatorname{sign} L_{1}+\operatorname{sign} L_{2},
\end{aligned}
$$

o que prova o lema.

Observe que, se $L_{1}: H_{1} \rightarrow H_{1}$ e $L_{2}: H_{2} \rightarrow H_{2}$ são isomorfismos auto-adjuntos, onde $H_{1}$ e $H_{2}$ são espaços de Hilbert de dimensão finita, então, de (3.2.1), o produto direto $\left(L_{1}, L_{2}\right): H_{1} \times H_{2} \rightarrow H_{1} \times H_{2}$ é um isomorfismo auto-adjunto. Como vimos em (3.1.2), a soma $H=\left[H_{1} \times\{0\}\right] \oplus\left[\{0\} \times H_{2}\right]$ é ortogonal. Neste caso, a matriz de operadores associada a $\left(L_{1}, L_{2}\right)$ e à decomposição de $H$ é da forma

$$
L=\left(\begin{array}{cc}
\hat{L}_{1} & 0 \\
0 & \hat{L}_{2}
\end{array}\right)
$$

onde $\hat{L}_{1}: H_{1} \times\{0\} \rightarrow H_{1} \times\{0\}$ é definido por $\hat{L}_{1}(x, 0)=\left(L_{1} x, 0\right)$ e $\hat{L}_{2}:\{0\} \times H_{2} \rightarrow$ $\{0\} \times H_{2}$ é definido por $\hat{L}_{2}(0, x)=\left(0, L_{2} x\right)$. Como consequência imediata do lema acima temos o seguinte corolário.

Corolário 4.1.6. Nas condições acima, $\operatorname{sign}\left(L_{1}, L_{2}\right)=\operatorname{sign} L_{1}+\operatorname{sign} L_{2}$.

Demonstração. Dos fatos mostrados acima e do Lema 4.1.5, obtemos que

$$
\operatorname{sign}\left(L_{1}, L_{2}\right)=\operatorname{sign} \hat{L}_{1}+\operatorname{sign} \hat{L}_{2}
$$

Não é difícil provar que $\operatorname{sign} \hat{L}_{1}=\operatorname{sign} L_{1}$ e $\operatorname{sign} \hat{L}_{2}=\operatorname{sign} L_{2}$. Consequentemente, $\operatorname{sign}\left(L_{1}, L_{2}\right)=\operatorname{sign} L_{1}+\operatorname{sign} L_{2}$.

Seja $L \in L(H)$ um isomorfismo auto-adjunto. O lema anterior mostra que, se $H=H_{1} \oplus H_{2}$, onde $H_{1}$ e $H_{2}$ são subespaços ortogonais de $H$ invariantes por $L$, então a assinatura de $L$ é igual à soma das assinaturas das restrições de $L$ aos espaços $H_{1}$ e $\mathrm{H}_{2}$. 
Antes de apresentar a próxima proposição, lembramos que, se $\left\{e_{1}, e_{2}, \ldots, e_{n}\right\}$ é uma base ortonormal de $H$, então, dado $x \in H$ e sua representação $x=\sum_{i=1}^{n} x_{i} e_{i}$, temos

$$
\|x\|=\sqrt{\sum_{i=1}^{n}\left|x_{i}\right|^{2}}
$$

De fato,

$$
\|x\|^{2}=\left\langle\sum_{i=1}^{n} x_{i} e_{i}, \sum_{j=1}^{n} x_{j} e_{j}\right\rangle=\sum_{i=1}^{n} x_{i}\left\langle e_{i}, \sum_{j=1}^{n} x_{j} e_{j}\right\rangle=\sum_{i=1}^{n} x_{i}\left\langle e_{i}, x_{i} e_{i}\right\rangle=\sum_{i=1}^{n}\left|x_{i}\right|^{2} .
$$

Finalizamos esta seção demonstrando que a aplicação assinatura é contínua.

Proposição 4.1.7. A aplicação sign : $G L_{S}(H) \rightarrow \mathbb{R}$ dada por

$$
L \mapsto \operatorname{sign} L
$$

é contínua.

Demonstração. Denotemos por $n$ a dimensão de $H$. Seja $L \in G L_{S}(H)$ fixado. Devemos mostrar que existe uma vizinhança $V \subseteq G L_{S}(H)$ de $L$ tal que, para todo $T \in V, \operatorname{sign} T=\operatorname{sign} L$. De fato, tomemos bases ortonormais $\left\{e_{1}, e_{2}, \ldots, e_{k}\right\}$ de $H_{+}(L)$ e $\left\{e_{k+1}, e_{k+2}, \ldots, e_{n}\right\}$ de $H_{-}(L)$ formadas por autovetores de $L$. Assim, para $i=1,2, \ldots, n$,

$$
L e_{i}=\lambda_{i} e_{i}, \quad \text { onde } \lambda_{i}>0 \text { para } i=1, \ldots, k \text { e } \lambda_{i}<0 \text { para } i=k+1, \ldots, n .
$$

Sejam $\lambda=\min \left\{\left|\lambda_{i}\right|: i=1,2, \ldots, n\right\}>0$ e $B(L, \lambda) \subseteq L_{S}(H)$ a bola com centro em $L$ e raio $\lambda$. Tomemos $T \in B(L, \lambda)$ fixado. Vejamos que $T$ é um isomorfismo e que $\operatorname{sign} T=\operatorname{sign} L$. De fato, para provar que $T$ é um isomorfismo é suficiente provar que $T$ é injetora, pois $H$ é de dimensão finita. Suponhamos por absurdo que exista $z \in H$ tal que $\|z\|=1$ e $T z=0$. Então, $z=\sum_{i=1}^{n} z_{i} e_{i}$, onde $z_{1}, z_{2}, \ldots, z_{n} \in \mathbb{R}$. De (4.1.4) temos $\left\|\sum_{i=1}^{n} \lambda_{i} z_{i} e_{i}\right\|^{2}=\sum_{i=1}^{n}\left|\lambda_{i} z_{i}\right|^{2}$, portanto

$$
\|T z-L z\|^{2}=\|L z\|^{2}=\left\|\sum_{i=1}^{n} \lambda_{i} z_{i} e_{i}\right\|^{2}=\sum_{i=1}^{n}\left|\lambda_{i} z_{i}\right|^{2} \geq|\lambda|^{2} \sum_{i=1}^{n}\left|z_{i}\right|^{2}=\lambda^{2}\|z\|^{2}=\lambda^{2} .
$$

Logo, $\|T-L\|=\sup _{\|x\|=1}\|T x-L x\| \geq \lambda$, contradizendo o fato de que $T \in B(L, \lambda)$. Assim, $T$ é injetor e portanto sobrejetor, isto é, $T$ é um isomorfismo. 
Agora provemos que $\operatorname{sign} T=\operatorname{sign} L$. Vejamos primeiro que $T$ é definida positiva em $H_{+}(L)$. Suponhamos por absurdo que $\langle T y, y\rangle \leq 0$ para algum $y=\sum_{i=1}^{k} y_{i} e_{i} \in H_{+}(L)$, com $\|y\|=1$. Daí,

$$
\begin{aligned}
\langle L y-T y, y\rangle & =\left\langle L\left(\sum_{i=1}^{k} y_{i} e_{i}\right)-T\left(\sum_{i=1}^{k} y_{i} e_{i}\right), \sum_{j=1}^{k} y_{j} e_{j}\right\rangle \\
& =\left\langle\sum_{i=1}^{k} \lambda_{i} y_{i} e_{i}, \sum_{j=1}^{k} y_{j} e_{j}\right\rangle-\left\langle T\left(\sum_{i=1}^{k} y_{i} e_{i}\right), \sum_{j=1}^{k} y_{j} e_{j}\right\rangle \\
& =\sum_{i=1}^{k} \lambda_{i}\left\langle y_{i} e_{i}, \sum_{j=1}^{k} y_{j} e_{j}\right\rangle-\langle T y, y\rangle \\
& \geq \sum_{i=1}^{k} \lambda_{i}\left\langle y_{i} e_{i}, y_{i} e_{i}\right\rangle \\
& =\sum_{i=1}^{k} \lambda_{i}\left|y_{i}\right|^{2} \\
& \geq \lambda \sum_{i=1}^{k}\left|y_{i}\right|^{2} \\
& =\lambda .
\end{aligned}
$$

Dado que $L-T$ é auto-adjunto, do Teorema 3.2.15 se segue

$$
\|L-T\|=\sup _{\|x\|=1}|\langle L x-T x, x\rangle| \geq \lambda
$$

contradizendo o fato de que $T \in B(L, \lambda)$. Portanto, $T$ é positivo em $H_{+}(L)$. Consequentemente, a soma $H_{+}(L)+H_{-}(T)$ é direta. Daí,

$$
\operatorname{dim} H_{-}(T) \leq \operatorname{dim} H_{-}(L) .
$$

Analogamente, $T$ é negativo em $H_{-}(L)$. Assim, a soma $H_{-}(L)+H_{+}(T)$ é direta. Então,

$$
\operatorname{dim} H_{+}(T) \leq \operatorname{dim} H_{+}(L)
$$

Como $T$ é um isomorfismo auto-adjunto, temos

$$
\operatorname{dim} H_{+}(T)+\operatorname{dim} H_{-}(T)=\operatorname{dim} H_{+}(L)+\operatorname{dim} H_{-}(L)=n .
$$

De (4.1.5), (4.1.6) e (4.1.7) se segue

$$
\operatorname{dim} H_{+}(T)=\operatorname{dim} H_{+}(L) \quad \text { e } \quad \operatorname{dim} H_{-}(T)=\operatorname{dim} H_{-}(L) .
$$

Logo, $\operatorname{sign} L=\operatorname{sign} T$. Em conclusão, sign é contínua.

Dado que a aplicação assinatura toma valores inteiros, da proposição anterior temos que esta aplicação é localmente constante. 


\subsection{A assinatura generalizada para perturbações compactas auto-adjuntas de uma simetria}

Nesta seção estenderemos a definição da assinatura, dada na seção anterior, para perturbações compactas auto-adjuntas de uma simetria em um espaço de Hilbert real, separável de dimensão infinita, lembrando, inclusive, a definição de simetria. Além disso, veremos algumas propriedades que possui esta extensão. A assinatura generalizada será definida fazendo uso da aproximação de Galerkin para operadores em um espaço de Hilbert separável. Mostraremos que esta generalizacão não é invariante pela ação cogradiente.

No resto do trabalho $H$ denotará um espaço de Hilbert real de dimensão infinita e separável. Denotaremos por $K_{S}(H)$ o espaço dos operadores compactos auto-adjuntos em $H$. Observe que $K_{S}(H)$ é um subespaço fechado de $L(H)$, pois é a interseção dos subespaços fechados $K(H)$ e $L_{S}(H)$.

Sejam $H_{-}$e $H_{+}$dois subespaços fechados de $H$, de dimensão infinita, ortogonais e tais que

$$
H=H_{+} \oplus H_{-} .
$$

Tomemos o operador $\mathcal{J} \in L(H)$ que tem a seguinte matriz de operadores associada à decomposição (4.2.1):

$$
\mathcal{J}=\left(\begin{array}{cc}
I_{H_{+}} & 0 \\
0 & -I_{H_{-}}
\end{array}\right)
$$

onde $I_{H_{+}}$e $I_{H_{-}}$são as identidades de $H_{+}$e $H_{-}$, respectivamente. É claro que $\mathcal{J}$ é um isomorfismo auto-adjunto. Além disso,

$$
H_{+}(\mathcal{J})=H_{+} \quad \text { e } \quad H_{-}(\mathcal{J})=H_{-} .
$$

Consequentemente, $\mathcal{J}$ é um operador fortemente indefinido, pois $H_{+}$e $H_{-}$têm dimensão infinita.

O operador $\mathcal{J}$ é uma simetria, isto é, $\mathcal{J}^{2}=I$. No resto do trabalho, se não temos ambiguidade, o termo simetria será usado para chamar operadores do tipo definido acima, denotados por $\mathcal{J}$, e, além disso, $H_{+}$e $H_{-}$denotarão os subespaços $H_{+}(\mathcal{J})$ e $H_{-}(\mathcal{J})$, respectivamente.

Sejam $\left(e_{i}^{+}\right)_{i=1}^{\infty}$ e $\left(e_{i}^{-}\right)_{i=1}^{\infty}$ bases de Hilbert de $H_{+}$e $H_{-}$, respectivamente. Logo,

$$
\mathcal{J} e_{i}^{+}=e_{i}^{+} \text {para todo } i \in \mathbb{N} \quad \text { e } \quad \mathcal{J} e_{i}^{-}=-e_{i}^{-} \text {para todo } i \in \mathbb{N} \text {. }
$$

Para cada número natural $n$, denotamos por $H_{n}$ o subespaço de $H$ de dimensão $2 n$ gerado por $\left(e_{i}^{ \pm}\right)_{i=1}^{n}$, isto é,

$$
H_{n}=\operatorname{span}\left\{e_{i}^{ \pm}: i=1, \ldots, n\right\}=\left\{x \in H: x=\sum_{i=1}^{n}\left(a_{i} e_{i}^{+}+b_{i} e_{i}^{-}\right), \text {onde } a_{i}, b_{i} \in \mathbb{R}\right\}
$$


Consequentemente, $\left(e_{i}^{ \pm}\right)_{i=n+1}^{\infty}$ se torna uma base de Hilbert para o espaço $H_{n}^{\perp}$. Assim, para cada número inteiro $k \geq 1$, temos

$$
H_{n+k} \cap H_{n}^{\perp}=\operatorname{span}\left\{e_{i}^{ \pm}: i=n+1, \ldots, n+k\right\} .
$$

Observação 4.2.1. É fácil provar que

$$
\mathcal{J}\left(H_{n}\right)=H_{n} \quad \text { e } \mathcal{J}\left(H_{n}^{\perp}\right)=H_{n}^{\perp} .
$$

Consideremos as restrições

$$
\left.\mathcal{J}\right|_{H_{n}}: H_{n} \rightarrow H_{n} \quad \text { e }\left.\quad \mathcal{J}\right|_{H_{n+k} \cap H_{n}^{\perp}}: H_{n+k} \cap H_{n}^{\perp} \rightarrow H_{n+k} \cap H_{n}^{\perp} .
$$

Da expressão dada em (4.2.2) temos

$$
H_{+}\left(\left.\mathcal{J}\right|_{H_{n}}\right)=\operatorname{span}\left\{e_{i}^{+}: i=1, \ldots, n\right\} \quad \text { e } \quad H_{-}\left(\left.\mathcal{J}\right|_{H_{n}}\right)=\operatorname{span}\left\{e_{i}^{-}: i=1, \ldots, n\right\} .
$$

Logo,

$$
\operatorname{sign}\left(\left.\mathcal{J}\right|_{H_{n}}\right)=\operatorname{dim}\left(H_{+}\left(\left.\mathcal{J}\right|_{H_{n}}\right)\right)-\operatorname{dim}\left(H_{-}\left(\left.\mathcal{J}\right|_{H_{n}}\right)\right)=0
$$

Por outro lado, as igualdades (4.2.2) e (4.2.3) implicam que

$$
H_{+}\left(\left.\mathcal{J}\right|_{H_{n+k} \cap H_{n}^{\perp}}\right)=\operatorname{span}\left\{e_{i}^{+}: i=n+1, \ldots, n+k\right\}
$$

e

$$
H_{-}\left(\left.\mathcal{J}\right|_{H_{n+k} \cap H_{n}^{\perp}}\right)=\operatorname{span}\left\{e_{i}^{-}: i=n+1, \ldots, n+k\right\}
$$

Portanto,

$$
\operatorname{sign}\left(\left.\mathcal{J}\right|_{H_{n+k} \cap H_{n}^{\perp}}\right)=0
$$

Como tínhamos falado acima, a seguir definiremos a assinatura de uma perturbação compacta auto-adjunta da simetria $\mathcal{J}$ usando a aproximação de Galerkin de operadores em espaços de Hilbert separáveis.

Definição 4.2.2. Seja $P_{n}$ a projeção ortogonal de $H$ sobre $H_{n}$. Para um operador $L \in L(H)$ e um inteiro positivo $n$, o operador $L_{n}: H_{n} \rightarrow H_{n}$ definido como a restrição de $P_{n} L$ ao subespaço $H_{n}$ é chamado de $n$-ésima aproximação de Galerkin de $L$.

Daqui à frente, se não se diz o contrario, a n-ésima aproximação de Galerkin será tomada a respeito dos subespaços $H_{n}$ e das bases $\left(e_{i}^{ \pm}\right)_{i=n+1}^{\infty}$ dadas anteriormente.

Observe que, se $x \in H$, então

$$
x=\sum_{n=1}^{\infty}\left(a_{n} e_{n}^{+}+b_{n} e_{n}^{-}\right)=\lim _{n \rightarrow+\infty} P_{n} x,
$$

isto é, a sequência $\left(P_{n}\right)_{n=1}^{\infty}$ converge pontualmente a $I$. Não é difícil provar que

$$
\left\|P_{n}-P_{m}\right\|=1 \text { para todo } n, m \in \mathbb{N}, \operatorname{com} n \neq m \text {. }
$$

Consequentemente, $\left(P_{n}\right)_{n=1}^{\infty}$ não converge a $I$ em $L(H)$. Porém, $\left(P_{n}\right)_{n=1}^{\infty}$ converge uniformemente a $I$ em qualquer subconjunto compacto de $H$. Para mostrar este fato, vejamos primeiro o seguinte lema. 
Lema 4.2.3. Sejam $\left(T_{n}\right)_{n=1}^{\infty}$ uma sequência de operadores em $L(E, F)$ e $T \in L(E, F)$, onde $E$ e $F$ são espaços de Banach. Suponhamos que exista $M>0$ tal que $\left\|T_{n}\right\| \leq M$ para todo $n \in \mathbb{N}$. Se $T_{n} x \rightarrow T x$ para todo $x \in E$, então $T_{n}$ converge uniformemente a $T$ em qualquer subconjunto compacto de $E$, isto é,

$$
\sup _{x \in C}\left\|T_{n} x-T x\right\| \rightarrow 0 \quad \text { quando } n \rightarrow \infty
$$

para qualquer subconjunto compacto $C$ de $E$.

Demonstração. Já que $C$ é um subconjunto compacto de $E$, ele é totalmente limitado (Teorema 1.4.2), isto é, para qualquer $\varepsilon>0$, existe um número finito de elementos $x_{1}, x_{2}, \ldots, x_{m}$ em $C$ tais que $C \subseteq \cup_{i=1}^{m} B\left(x_{i}, \varepsilon\right)$, onde $B\left(x_{i}, \varepsilon\right)$ é a bola com centro em $x_{i}$ e raio $\varepsilon$. Dado que, para $i=1,2, \ldots m, T_{n} x_{i} \rightarrow T x_{i}$ quando $n \rightarrow \infty$, existe um número $n_{i} \in \mathbb{N}$, em correspondência a $x_{i}$, tal que

$$
\left\|T_{n} x_{i}-T x_{i}\right\|<\varepsilon \quad \text { para } n>n_{i} .
$$

Sejam $x \in C$ e $n>\max \left\{n_{i}: i=1,2, \ldots, m\right\}$. Como $C \subseteq \cup_{i=1}^{m} B\left(x_{i}, \varepsilon\right)$, temos que $\left\|x-x_{i}\right\|<\varepsilon$ para algum $x_{i}$. Consequentemente,

$$
\begin{aligned}
\left\|T_{n} x-T x\right\| & \leq\left\|T_{n} x-T_{n} x_{i}\right\|+\left\|T_{n} x_{i}-T x_{i}\right\|+\left\|T x_{i}-T x\right\| \\
& \leq\left\|T_{n}\right\|\left\|x-x_{i}\right\|+\varepsilon+\|T\|\left\|x-x_{i}\right\| \\
& \leq M \varepsilon+\varepsilon+\|T\| \varepsilon \\
& =(M+1+\|T\|) \varepsilon .
\end{aligned}
$$

Logo,

$$
\sup _{x \in C}\left\|T_{n} x-T x\right\| \rightarrow 0 \quad \text { quando } n \rightarrow \infty
$$

o que prova o lema.

Dado que cada uma das projeções $P_{n}$ tem norma 1 e que a sequência $\left(P_{n}\right)_{n=1}^{\infty}$ converge pontualmente a $I$, se segue do lema anterior que esta sequência converge uniformemente a $I$ em qualquer subconjunto compacto de $H$. O seguinte lema é uma consequência do fato anterior.

Lema 4.2.4. Se $K$ é um operador compacto, a sequência $\left(P_{n} K\right)_{n=1}^{\infty}$ converge uniformemente a $K$ em $L(H)$.

Demonstração. Tomemos $C=\overline{K(B)}$, onde $B$ é a bola de raio 1 e centro em $0 \in H$. Seja $\varepsilon>0$ fixado. Dado que $C$ é compacto, do lema anterior se segue que existe um inteiro positivo $N$ tal que, se $n \geq N$, então $\sup _{y \in C}\left\|y-P_{n} y\right\|<\varepsilon$. Portanto, se $n \geq N$,

$$
\begin{aligned}
\left\|K-P_{n} K\right\| & =\sup _{x \in B}\left\|\left(K-P_{n} K\right) x\right\|=\sup _{x \in B}\left\|\left(I-P_{n}\right) K x\right\| \\
& =\sup _{y \in K(B)}\left\|\left(I-P_{n}\right) y\right\|=\sup _{y \in C}\left\|y-P_{n} y\right\|<\varepsilon .
\end{aligned}
$$

Este fato implica que $\left(P_{n} K\right)_{n=1}^{\infty}$ converge uniformemente a $K$ em $L(H)$. 
A proposição que apresentaremos a seguir é fundamental para a definição da assinatura de uma perturbação compacta auto-adjunta da simetria $\mathcal{J}$.

Proposição 4.2.5. Sejam $L=\mathcal{J}+K \in G L_{S}(H)$ uma perturbação compacta autoadjunta de $\mathcal{J}$ e, para $n$ natural, $L_{n}$ a n-ésima aproximação de Galerkin de L. Então, existe um inteiro positivo $N$ tal que $\operatorname{sign} L_{n}$ é independente de $n \geq N$.

Demonstração. Provemos que existe um inteiro positivo $N$ tal que, para $n \geq m \geq N$ e $0 \leq t \leq 1$, o operador

$$
\mathcal{J}_{n, m}(t)=\left.t\left[\mathcal{J}+P_{n} K\right]\right|_{H_{n}}+\left.(1-t)\left[\mathcal{J}+P_{m} K P_{m}\right]\right|_{H_{n}}: H_{n} \rightarrow H_{n}
$$

é inversível, onde $H_{n}$ é o subespaço definido acima. Supondo por absurdo que não exista tal $N$, então, para cada $k \in \mathbb{N}$, existem $t_{k} \in[0,1]$ e duas sequências estritamente crescentes de inteiros positivos, $\left(m_{k}\right)_{k=1}^{\infty}$ e $\left(n_{k}\right)_{k=1}^{\infty}$, tais que $n_{k} \geq m_{k}$ e, além disso,

$$
\mathcal{J}_{n_{k}, m_{k}}(t): H_{n_{k}} \rightarrow H_{n_{k}}
$$

não é inversível. Logo, para cada $k$, existe um vetor $u_{n_{k}}$ em $H_{n_{k}}$, que podemos pegar sem perda de generalidade de norma 1 , tal que

$$
t_{k} \mathcal{J} u_{n_{k}}+P_{n_{k}} K u_{n_{k}}+\left(1-t_{k}\right) \mathcal{J} u_{n_{k}}+\left(1-t_{k}\right) P_{m_{k}} K P_{m_{k}} u_{n_{k}}=0 .
$$

Assim,

$$
\mathcal{J} u_{n_{k}}+t_{k} P_{n_{k}} K u_{n_{k}}+\left(1-t_{k}\right) P_{m_{k}} K P_{m_{k}} u_{n_{k}}=0 \text { para todo } k .
$$

Pela compacidade de $[0,1]$, podemos supor que $t_{k} \rightarrow t \in[0,1]$.

O Teorema 2.3.10 implica que

$$
-t \mathcal{J}^{-1} K-(1-t) \mathcal{J}^{-1} K=-\mathcal{J}^{-1} K
$$

é um operador compacto. Logo, da Proposição 2.3.4 obtemos que

$$
-t \mathcal{J}^{-1} K u_{n_{k}}-(1-t) \mathcal{J}^{-1} K u_{n_{k}}=-\mathcal{J}^{-1} K u_{n_{k}}
$$

possui uma subsequência, à qual podemos dar o mesmo nome sem perda de generalidade, convergente a algum $u \in H$.

Como consequência do Lema 4.2.4 temos que $-t_{k} \mathcal{J}^{-1} P_{n_{k}} K-\left(1-t_{k}\right) \mathcal{J}^{-1} P_{m_{k}} K P_{m_{k}}$ converge uniformemente a $-t \mathcal{J}^{-1} K-(1-t) \mathcal{J}^{-1} K$. Daí, dado $\varepsilon>0$, existe um inteiro positivo $N$ tal que, se $k \geq N$,

$$
\left\|-t \mathcal{J}^{-1} K-(1-t) \mathcal{J}^{-1} K-\left(-t_{k} \mathcal{J}^{-1} P_{n_{k}} K-\left(1-t_{k}\right) \mathcal{J}^{-1} P_{m_{k}} K P_{m_{k}}\right)\right\|<\varepsilon .
$$


Logo, como $-t \mathcal{J}^{-1} K-(1-t) \mathcal{J}^{-1} K=-\mathcal{J}^{-1} K$,

$$
\begin{aligned}
\|-\mathcal{J}^{-1} K & u_{n_{k}}-\left(-t_{k} \mathcal{J}^{-1} P_{n_{k}} K-\left(1-t_{k}\right) \mathcal{J}^{-1} P_{m_{k}} K P_{m_{k}}\right) u_{n_{k}} \| \\
& \leq\left\|-\mathcal{J}^{-1} K-\left(-t_{k} \mathcal{J}^{-1} P_{n_{k}} K-\left(1-t_{k}\right) \mathcal{J}^{-1} P_{m_{k}} K P_{m_{k}}\right)\right\|\left\|u_{n_{k}}\right\| \\
& =\left\|-\mathcal{J}^{-1} K-\left(-t_{k} \mathcal{J}^{-1} P_{n_{k}} K-\left(1-t_{k}\right) \mathcal{J}^{-1} P_{m_{k}} K P_{m_{k}}\right)\right\| \\
& <\varepsilon
\end{aligned}
$$

Este fato implica que $-t_{k} \mathcal{J}^{-1} P_{n_{k}} K u_{n_{k}}-\left(1-t_{k}\right) \mathcal{J}^{-1} P_{m_{k}} K P_{m_{k}} u_{n_{k}}$ converge a $u$. De (4.2.5) se segue que

$$
u_{n_{k}}=-t_{k} \mathcal{J}^{-1} P_{n_{k}} K u_{n_{k}}-\left(1-t_{k}\right) \mathcal{J}^{-1} P_{m_{k}} K P_{m_{k}} u_{n_{k}} \text { para todo } k .
$$

Assim, $u_{n_{k}}$ converge a $u$, que se torna portanto de norma 1 .

Do Lema 4.2.4 obtemos que a sequência $\mathcal{J}+t_{k} P_{n_{k}} K+\left(1-t_{k}\right) P_{m_{k}} K P_{m_{k}}$ converge uniformemente a $\mathcal{J}+t K+(1-t) K=\mathcal{J}+K$. Portanto, dado que $u_{n_{k}}$ converge a $u$,

$$
\mathcal{J} u_{n_{k}}+t_{k} P_{n_{k}} K u_{n_{k}}+\left(1-t_{k}\right) P_{m_{k}} K P_{m_{k}} u_{n_{k}} \quad \text { converge a } \quad \mathcal{J} u+K u .
$$

Já que $\mathcal{J} u_{n_{k}}+t_{k} P_{n_{k}} K u_{n_{k}}+\left(1-t_{k}\right) P_{m_{k}} K P_{m_{k}} u_{n_{k}}=0$ para todo $k$, então $\mathcal{J} u+K u=0$, contradizendo o fato de que $\mathcal{J}+K$ é inversível. Em conclusão, existe $N>0$ tal que, para $n \geq m \geq N$ e $0 \leq t \leq 1$, o operador $\mathcal{J}_{n, m}(t)$ definido em (4.2.4) é um isomorfismo.

Consideremos portanto um inteiro positivo $N$ tal que, para $n \geq m \geq N$ e $0 \leq t \leq 1$, o operador $\mathcal{J}_{n, m}(t)$ definido na fórmula (4.2.4) seja inversível. Sejam $n \geq m \geq N$ números fixos. Os operadores

$$
\left.\left[\mathcal{J}+P_{n} K\right]\right|_{H_{n}}=\left.\left[\mathcal{J}+P_{n} K P_{n}\right]\right|_{H_{n}}: H_{n} \rightarrow H_{n} \quad \text { e }\left.\quad\left[\mathcal{J}+P_{m} K P_{m}\right]\right|_{H_{n}}: H_{n} \rightarrow H_{n}
$$

são auto-adjuntos, pois $\mathcal{J}, P_{n} K P_{n}$ e $P_{m} K P_{m}$ são auto-adjuntos. Consequentemente, $\mathcal{J}_{n, m}(t): H_{n} \rightarrow H_{n}$ é um caminho de isomorfismos auto-adjuntos. Pela continuidade da assinatura se segue sign $\mathcal{J}_{n, m}(0)=\operatorname{sign} \mathcal{J}_{n, m}(1)$, isto é,

$$
\operatorname{sign}\left(\left.\left[\mathcal{J}+P_{m} K P_{m}\right]\right|_{H_{n}}\right)=\operatorname{sign}\left(\left.\left[\mathcal{J}+P_{n} K\right]\right|_{H_{n}}\right)=\operatorname{sign}\left(\left.\left[P_{n}(\mathcal{J}+K)\right]\right|_{H_{n}}\right)=\operatorname{sign} L_{n} .
$$

É claro que $H_{m}$ e $H_{n} \cap H_{m}^{\perp}$ são invariantes por $\mathcal{J}+P_{m} K P_{m}$, pois são invariantes por $\mathcal{J}$ e $P_{m} K P_{m}$. Como $H_{n}=H_{m} \oplus\left[H_{n} \cap H_{m}^{\perp}\right]$, do Lema 4.1.5 e da Observação 4.2.1 temos

$$
\begin{aligned}
\operatorname{sign}\left(\left.\left[\mathcal{J}+P_{m} K P_{m}\right]\right|_{H_{n}}\right) & =\operatorname{sign}\left(\left.\left[\mathcal{J}+P_{m} K P_{m}\right]\right|_{H_{m}}\right)+\operatorname{sign}\left(\left.\left[\mathcal{J}+P_{m} K P_{m}\right]\right|_{H_{n} \cap H_{\frac{1}{m}}}\right) \\
& =\operatorname{sign} L_{m}+\operatorname{sign}\left(\left.[\mathcal{J}]\right|_{H_{n} \cap H_{m}^{\perp}}+\left.\left[P_{m} K P_{m}\right]\right|_{H_{n} \cap H_{m}^{\perp}}\right) \\
& =\operatorname{sign} L_{m}+\operatorname{sign}\left(\left.[\mathcal{J}]\right|_{H_{n} \cap H_{m}^{\perp}}\right) \\
& =\operatorname{sign} L_{m} .
\end{aligned}
$$

Assim, $\operatorname{sign} L_{n}=\operatorname{sign} L_{m}$ para $n \geq m \geq N$, o que prova a proposição. 
Como consequência obtemos a seguinte definição.

Definição 4.2.6. Seja $L=\mathcal{J}+K \in G L(H)$ uma perturbação compacta auto-adjunta de $\mathcal{J}$. Então, sua assinatura, denotada por $\operatorname{sign}_{\mathcal{J}}\left(L,\left(e_{i}{ }^{ \pm}\right)_{i=1}^{\infty}\right)$, é definida por

$$
\operatorname{sign}_{\mathcal{J}}\left(L,\left(e_{i}{ }^{ \pm}\right)_{i=1}^{\infty}\right)=\lim _{n \rightarrow \infty} \operatorname{sign} L_{n}
$$

Provemos que a Definição 4.2.6 não depende da escolha das bases de $H_{+}$e $H_{-}$. De fato, sejam $\left(\widetilde{e}_{i}^{+}\right)_{i=1}^{\infty}$ e $\left(\widetilde{e}_{i}^{-}\right)_{i=1}^{\infty}$ outras bases de Hilbert de $H_{+}$e $H_{-}$, respectivamente. Sejam $O_{+}: H_{+} \rightarrow H_{+}$e $O_{-}: H_{-} \rightarrow H_{-}$os operadores ortogonais definidos por

$$
O_{+} \widetilde{e}_{i}^{+}=e_{i}^{+} \quad \text { e } \quad O_{-} \widetilde{e}_{i}^{-}=e_{i}^{-} \text {para todo } i \in \mathbb{N} .
$$

Tomemos $O \in L(H)$ definido por

$$
O \widetilde{e}_{i}^{ \pm}=O_{ \pm} \widetilde{e}_{i}^{ \pm}=e_{i}^{ \pm} \text {para todo } i \in \mathbb{N}
$$

Não é difícil provar que $O$ é ortogonal.

Mostremos que $O^{-1} \mathcal{J} O=\mathcal{J}$. De fato, seja $x=\sum_{i=1}^{\infty}\left(a_{i} \widetilde{e}_{i}^{+}+b_{i} \widetilde{e}_{i}^{-}\right) \in H$ fixado. Daí,

$$
\begin{aligned}
O^{-1} \mathcal{J} O x & =O^{-1} \mathcal{J}\left(O \sum_{i=1}^{\infty}\left(a_{i} \widetilde{e}_{i}^{+}+b_{i} \widetilde{e}_{i}^{-}\right)\right)=O^{-1} \mathcal{J}\left(\sum_{i=1}^{\infty}\left(a_{i} e_{i}^{+}+b_{i} e_{i}^{-}\right)\right) \\
& =O^{-1}\left(\sum_{i=1}^{\infty}\left(a_{i} e_{i}^{+}-b_{i} e_{i}^{-}\right)\right)=\sum_{i=1}^{\infty}\left(a_{i} \widetilde{e}_{i}^{+}-b_{i} \widetilde{e}_{i}^{-}\right) \\
& =\mathcal{J}\left(\sum_{i=1}^{\infty}\left(a_{i} \widetilde{e}_{i}^{+}+b_{i} \widetilde{e}_{i}^{-}\right)\right)=\mathcal{J} x
\end{aligned}
$$

isto é, $O^{-1} \mathcal{J} O x=\mathcal{J} x$ para todo $x \in H$.

Denotemos por $\widetilde{H}_{n}$ o espaço gerado por $\left\{\widetilde{e}_{k}^{ \pm}: 1 \leq k \leq n\right\}$ e por $\widetilde{P}_{n}$ a projeção ortogonal sobre $\widetilde{H}_{n}$. Da definição de $O$ temos $H_{n}=O\left(\widetilde{H}_{n}\right)$. Logo, as restrições $O: \widetilde{H}_{n} \rightarrow H_{n}$ e $O^{-1}: H_{n} \rightarrow \widetilde{H}_{n}$ são bem definidas e, além disso, isomorfismos.

Observe que $\left.O \widetilde{P}_{n}\right|_{\widetilde{H}_{n}}=\left.P_{n} O\right|_{\widetilde{H}_{n}}$. De fato, se $x \in \widetilde{H}_{n}$, então $O x \in H_{n}$. Consequentemente,

$$
O \widetilde{P}_{n} x=O x=P_{n} O x \quad \text { para todo } x \in \widetilde{H}_{n} .
$$

Por outro lado, vejamos que $O^{-1} P_{n}=\widetilde{P}_{n} O^{-1}$. Seja $x \in H$ fixado. Daí, $x=$ $x_{1}+x_{2} \in H_{n} \oplus H_{n}^{\perp}$, onde $x_{1} \in H_{n}$ e $x_{2} \in H_{n}^{\perp}$. Portanto, $O^{-1} x_{1} \in \widetilde{H}_{n}$ e $O^{-1} x_{2} \in \widetilde{H}_{n}^{\perp}$. Assim,

$$
\widetilde{P}_{n} O^{-1}\left(x_{1}+x_{2}\right)=\widetilde{P}_{n} O^{-1} x_{1}+\widetilde{P}_{n} O^{-1} x_{2}=O^{-1} x_{1}=O^{-1} P_{n} x_{1}=O^{-1} P_{n}\left(x_{1}+x_{2}\right),
$$

o que prova que $O^{-1} P_{n} x=\widetilde{P}_{n} O^{-1} x$. 
Dado que $O^{-1}=O^{*}$, o operador compacto $O^{-1} K O$ é auto-adjunto. Logo, da Proposição 4.2.5 se segue que existe $n$ suficientemente grande tal que

$$
\operatorname{sign}_{\mathcal{J}}\left(\mathcal{J}+K,\left(e_{i}{ }^{ \pm}\right)_{i=1}^{\infty}\right)=\operatorname{sign}\left(\left.\left[\mathcal{J}+P_{n} K P_{n}\right]\right|_{H_{n}}\right)
$$

e

$$
\operatorname{sign}_{\mathcal{J}}\left(\mathcal{J}+O^{-1} K O,\left(\widetilde{e}_{i}^{ \pm}\right)_{i=1}^{\infty}\right)=\operatorname{sign}\left(\left.\left[\mathcal{J}+\widetilde{P}_{n} O^{-1} K O \widetilde{P}_{n}\right]\right|_{\widetilde{H}_{n}}\right) .
$$

Já que $O^{-1} P_{n}=\widetilde{P}_{n} O^{-1}$ e $\left.O \widetilde{P}_{n}\right|_{\widetilde{H}_{n}}=\left.P_{n} O\right|_{\widetilde{H}_{n}}$, do Lema 4.1 .4 temos

$$
\begin{aligned}
\operatorname{sign}\left(\left.\left[\mathcal{J}+\widetilde{P}_{n} O^{-1} K O \widetilde{P}_{n}\right]\right|_{\widetilde{H}_{n}}\right) & =\operatorname{sign}\left(\left.\left[\mathcal{J}+O^{-1} P_{n} K P_{n} O\right]\right|_{\widetilde{H}_{n}}\right) \\
& =\operatorname{sign}\left(\left.O^{-1}\left[\mathcal{J}+P_{n} K P_{n}\right] O\right|_{\widetilde{H}_{n}}\right) \\
& =\operatorname{sign}\left(\left.\left[\mathcal{J}+P_{n} K P_{n}\right]\right|_{H_{n}}\right),
\end{aligned}
$$

isto é,

$$
\operatorname{sign}\left(\left.\left[\mathcal{J}+\widetilde{P}_{n} O^{-1} K O \widetilde{P}_{n}\right]\right|_{\widetilde{H}_{n}}\right)=\operatorname{sign}\left(\left.\left[\mathcal{J}+P_{n} K P_{n}\right]\right|_{H_{n}}\right) .
$$

Como na prova da Proposição 4.2.5, temos que existe $N \in \mathbb{N}$ tal que, se $n \geqslant N$ e $0 \leqslant t \leqslant 1$

$$
\mathcal{J}+t \widetilde{P}_{n} K \widetilde{P}_{n}+(1-t) \widetilde{P}_{n} O^{-1} K O \widetilde{P}_{n}: \widetilde{H}_{n} \rightarrow \widetilde{H}_{n}
$$

é um isomorfismo auto-adjunto. A continuidade da assinatura (ver Proposição 4.1.7) implica que

$$
\operatorname{sign}\left(\left.\left[\mathcal{J}+\widetilde{P}_{n} K \widetilde{P}_{n}\right]\right|_{\widetilde{H}_{n}}\right)=\operatorname{sign}\left(\left.\left[\mathcal{J}+\widetilde{P}_{n} O^{-1} K O \widetilde{P}_{n}\right]\right|_{\widetilde{H}_{n}}\right) .
$$

Assim, de (4.2.7), (4.2.8) e (4.2.9) temos

$$
\operatorname{sign}_{\mathcal{J}}\left(\mathcal{J}+K,\left(e_{i}{ }^{ \pm}\right)_{i=1}^{\infty}\right)=\operatorname{sign}\left(\left.\left[\mathcal{J}+P_{n} K P_{n}\right]\right|_{H_{n}}\right)=\operatorname{sign}\left(\left.\left[\mathcal{J}+\widetilde{P}_{n} K \widetilde{P}_{n}\right]\right|_{\widetilde{H}_{n}}\right),
$$

isto é,

$$
\operatorname{sign}_{\mathcal{J}}\left(\mathcal{J}+K,\left(e_{i}{ }^{ \pm}\right)_{i=1}^{\infty}\right)=\operatorname{sign}_{\mathcal{J}}\left(\mathcal{J}+K,\left(\widetilde{e}_{i}^{ \pm}\right)_{i=1}^{\infty}\right)
$$

Este fato prova que a Definição 4.2.6 não depende das bases escolhidas para $H_{+}$e $H_{-}$, respectivamente. Consequentemente, daqui à frente denotaremos simplesmente por

$$
\operatorname{sign}_{\mathcal{J}}(\mathcal{J}+K)
$$

a assinatura generalizada do operador $\mathcal{J}+K$.

O seguinte resultado é consequência da independência da assinatura da escolha das bases de $H_{+}$e $H_{-}$.

Proposição 4.2.7. Seja $K \in L(H)$ um operador auto-adjunto com imagem de dimensão finita. Suponhamos que $V$ seja um subespaço de dimensão finita de $H$, invariante por $\mathcal{J}$ e tal que $\operatorname{Im} K \subseteq V$. Então,

$$
\operatorname{sign}_{\mathcal{J}} L=\operatorname{sign}\left(\left.[\mathcal{J}+K]\right|_{V}\right)-\operatorname{sign}\left(\left.[\mathcal{J}]\right|_{V}\right)
$$

onde $L=\mathcal{J}+K$. 
Demonstração. De fato, tomemos uma base $\left\{v_{1}, v_{2}, \ldots, v_{s}\right\}$ de $V$. Consideremos bases de Hilbert $\left(e_{i}^{ \pm}\right)_{i=1}^{\infty}$ de $H_{ \pm}$. Assim,

$$
v_{k}=\sum_{i=1}^{\infty} x_{i}^{k} e_{i}^{+}+\sum_{i=1}^{\infty} y_{i}^{k} e_{i}^{-} \quad \text { para } \quad k=1,2, \ldots, s .
$$

Sejam

$$
v_{k}^{+}=\sum_{i=1}^{\infty} x_{i}^{k} e_{i}^{+} \in H_{+} \quad \text { e } \quad v_{k}^{-}=\sum_{i=1}^{\infty} y_{i}^{k} e_{i}^{-} \in H_{-} \quad \text { para } \quad k=1,2, \ldots, s .
$$

Pelo processo de ortogonalização de Gram-Schmidt, para $k=1,2, \ldots, s$, existem vetores ortonormais $u_{k}^{+} \in H_{+}$e $u_{k}^{-} \in H_{-}$tais que

$$
\operatorname{span}\left\{u_{k}^{ \pm}: k=1,2, \ldots, s\right\}=\operatorname{span}\left\{v_{k}^{ \pm}: k=1,2 \ldots, s\right\} .
$$

Portanto, $V$ é um subespaço de $\widetilde{H}_{s}=\widetilde{H}_{+}^{s} \oplus \widetilde{H}_{-}^{s}$, onde

$$
\widetilde{H}_{ \pm}^{s}=\operatorname{span}\left\{u_{k}^{ \pm}: k=1, \ldots, s\right\} .
$$

Podemos estender as bases de $\widetilde{H}_{ \pm}^{s}$ a bases de Hilbert de $H_{ \pm}$. Denotaremos por $\widetilde{H}_{n}$ o espaço gerado pelos $n$ primeiros termos das novas bases de $H_{ \pm}$. Seja $n \geq s$ tal que

$$
\operatorname{sign}_{\mathcal{J}}(\mathcal{J}+K)=\operatorname{sign}\left(\left.[\mathcal{J}+K]\right|_{\widetilde{H}_{n}}\right) .
$$

Ponhamos $V_{n}^{\perp}=V^{\perp} \cap \widetilde{H}_{n}$. Assim, $\widetilde{H}_{n}=V \oplus V_{n}^{\perp}$. Observe que $V_{n}^{\perp}$ é invariante por $\mathcal{J}$. De fato, seja $x \in V_{n}^{\perp}$ fixado. Dado que $V$ é invariante por $\mathcal{J}$, para todo $y \in V$, temos que $\mathcal{J} y \in V$. Daí,

$$
\langle\mathcal{J} x, y\rangle=\left\langle x, \mathcal{J}^{*} y\right\rangle=\langle x, \mathcal{J} y\rangle=0 \quad \text { para todo } y \in V,
$$

isto é, $\mathcal{J} x \in V^{\perp} \cap \widetilde{H}_{n}=V_{n}^{\perp}$.

Sejam $P_{V}: \widetilde{H}_{n} \rightarrow V$ e $P_{V_{n}^{\perp}}: \widetilde{H}_{n} \rightarrow V_{n}^{\perp}$ as projeções ortogonais sobre $V$ e $V_{n}^{\perp}$, respectivamente. Se $x \in \widetilde{H}_{n}$, então $x=v+v^{\perp}$, onde $v \in V$ e $v^{\perp} \in V_{n}^{\perp}$. Já que $V$ e $V_{n}^{\perp}$ são invariantes por $\mathcal{J}$, se segue que $P_{V_{n}^{\perp}} \mathcal{J} v=0$ e $P_{V} \mathcal{J} v^{\perp}=0$. Como a imagem de $K$ está contida em $V$, temos que $P_{V_{n}^{\perp}} K v=0$ e $P_{V_{n}^{\perp}} K v^{\perp}=0$. Então,

$$
\begin{aligned}
(\mathcal{J}+K) x & =\left(P_{V}+P_{V_{n}^{\perp}}\right)(\mathcal{J}+K)\left(P_{V}+P_{V_{n}^{\perp}}\right) x \\
& =P_{V}(\mathcal{J}+K) v+P_{V}(\mathcal{J}+K) v^{\perp}+P_{V_{n}^{\perp}}(\mathcal{J}+K) v+P_{V_{n}^{\perp}}(\mathcal{J}+K) v^{\perp} \\
& =P_{V}(\mathcal{J}+K) v+P_{V} K v^{\perp}+P_{V_{n}^{\perp}} \mathcal{J} v^{\perp} .
\end{aligned}
$$

Se segue do Corolário 3.2.10 que $K v^{\perp}=0$. Daí,

$$
(\mathcal{J}+K)\left(v+v^{\perp}\right)=P_{V}(\mathcal{J}+K) v+P_{V_{n}^{\perp}} \mathcal{J} v^{\perp} .
$$


Logo, a matriz de operadores da restrição $\left.[\mathcal{J}+K]\right|_{\widetilde{H}_{n}}: \widetilde{H}_{n} \rightarrow \widetilde{H}_{n}$ associada à decomposição $\widetilde{H}_{n}=V \oplus V_{n}^{\perp}$ é dada por

$$
\left.[\mathcal{J}+K]\right|_{\widetilde{H}_{n}}=\left(\begin{array}{cc}
{\left.[\mathcal{J}+K]\right|_{V}} & 0 \\
0 & {\left.[\mathcal{J}]\right|_{V_{n}^{\perp}}}
\end{array}\right) .
$$

O Lema 4.1.5 implica que

$$
\operatorname{sign}\left(\left.[\mathcal{J}+K]\right|_{\widetilde{H}_{n}}\right)=\operatorname{sign}\left(\left.[\mathcal{J}+K]\right|_{V}\right)+\operatorname{sign}\left(\left.[\mathcal{J}]\right|_{V_{n}^{\perp}}\right) .
$$

Da Observação 4.2.1 e do Lema 4.1.5 segue-se

$$
0=\operatorname{sign}\left(\left.[\mathcal{J}]\right|_{\widetilde{H}_{n}}\right)=\operatorname{sign}\left(\left.[\mathcal{J}]\right|_{V}\right)+\operatorname{sign}\left(\left.[\mathcal{J}]\right|_{V_{n}^{\perp}}\right) .
$$

Consequentemente,

$$
\operatorname{sign}_{\mathcal{J}} L=\operatorname{sign}\left(\left.[\mathcal{J}+K]\right|_{\widetilde{H}_{n}}\right)=\operatorname{sign}\left(\left.[\mathcal{J}+K]\right|_{V}\right)-\operatorname{sign}\left(\left.[\mathcal{J}]\right|_{V}\right),
$$

o que prova a proposição.

Se um operador $L$ pode ser escrito como uma perturbação auto-adjunta compacta de duas simetrias $\mathcal{J}$ e $\mathcal{J}^{\prime}$ poderíamos ter $\operatorname{sign}_{\mathcal{J}^{\prime}} L \neq \operatorname{sign}_{\mathcal{J}} L$. Este fato é mostrado no seguinte exemplo, onde, inclusive, provamos que $\operatorname{sign}_{\mathcal{J}} L$ não é invariante pela ação cogradiente.

Exemplo 4.2.8. Seja $\mathcal{J}^{\prime} \in \Phi_{S}^{i}(H)$ uma simetria tal que, para uma base de Hilbert $\left\{e_{i}:-\infty<i<\infty\right\}$ de $H$,

$$
H_{+}\left(\mathcal{J}^{\prime}\right)=\operatorname{span}\left\{e_{i}: i \geq 0\right\} \quad \text { e } \quad H_{-}\left(\mathcal{J}^{\prime}\right)=\operatorname{span}\left\{e_{i}: i \leq-1\right\} .
$$

Seja $S: H \rightarrow H$ definido por

$$
S e_{i}=e_{i-1} \quad \text { para }-\infty<i<\infty .
$$

Observe que $S$ é ortogonal ( $S$ leva base ortonormal a base ortonormal). Tomemos $\mathcal{J}^{\prime \prime}=S^{*} \mathcal{J}^{\prime} S$. Assim,

$$
\mathcal{J}^{\prime \prime} e_{i}=e_{i} \text { se } i \geq 1 \quad \text { e } \quad \mathcal{J}^{\prime \prime} e_{i}=-e_{i} \text { se } i \leq 0 .
$$

Portanto, $\mathcal{J}^{\prime \prime}$ é uma simetria em $\Phi_{S}^{i}(H)$, onde

$$
H_{+}\left(\mathcal{J}^{\prime \prime}\right)=\left\{e_{i}: i \geq 1\right\} \quad \text { e } \quad H_{-}\left(\mathcal{J}^{\prime \prime}\right)=\left\{e_{i}: i \leq 0\right\} .
$$

Seja $K: H \rightarrow H$ o operador tal que

$$
K e_{0}=-2 e_{0} \quad \text { e } \quad K e_{i}=0 \text { para } i \in \mathbb{Z}-\{0\} .
$$


É fácil ver que $\mathcal{J}^{\prime}=\mathcal{J}^{\prime \prime}-K$. A imagem de $K$, que é o subespaço $H_{0}=\operatorname{span}\left\{e_{0}\right\}$, é invariante por $\mathcal{J}^{\prime \prime}$. Da Proposição 4.2.7 se segue

$$
\operatorname{sign}_{\mathcal{J}^{\prime \prime}} \mathcal{J}^{\prime}=\operatorname{sign}_{\mathcal{J}^{\prime \prime}}\left(\mathcal{J}^{\prime \prime}-K\right)=\operatorname{sign}\left(\left.\left[\mathcal{J}^{\prime \prime}-K\right]\right|_{H_{0}}\right)-\operatorname{sign}\left(\left.\left[\mathcal{J}^{\prime \prime}\right]\right|_{H_{0}}\right) .
$$

Como $\left(\mathcal{J}^{\prime \prime}-K\right) e_{0}=\mathcal{J}^{\prime} e_{0}=e_{0}$, então $\operatorname{sign}\left(\left.\left[\mathcal{J}^{\prime \prime}-K\right]\right|_{H_{0}}\right)=1$. Agora, $\mathcal{J}^{\prime \prime} e_{0}=-e_{0}$. Assim, $\operatorname{sign}\left(\left.\left[\mathcal{J}^{\prime \prime}\right]\right|_{H_{0}}\right)=-1$. Daí,

$$
\operatorname{sign}_{\mathcal{J}^{\prime \prime}} \mathcal{J}^{\prime}=2 \neq 0=\operatorname{sign}_{\mathcal{J}^{\prime \prime}} \mathcal{J}^{\prime \prime}
$$

Na Proposição 4.2.11 apresentaremos uma outra propriedade da assinatura generalizada, que tem a ver com o produto direto de dois operadores em espaços de Hilbert. Para este fim, vejamos primeiro os seguintes resultados.

Sejam $H_{1}$ e $H_{2}$ dois espaços de Hilbert reais. Consideremos o espaço de Hilbert $H_{1} \times H_{2}$ com o produto interno definido em (3.1.1). De (3.2.1) temos que, se $L_{1} \in L\left(H_{1}\right)$ e $L_{2} \in L\left(H_{2}\right)$, então $\left(L_{1}, L_{2}\right)^{*}=\left(L_{1}^{*}, L_{2}^{*}\right)$.

Lema 4.2.9. Se $L_{1} \in L\left(H_{1}\right)$ e $L_{2} \in L\left(H_{2}\right)$ são operadores auto-adjuntos, então

$$
\begin{gathered}
H_{+}\left(L_{1}, L_{2}\right)=H_{+}\left(L_{1}\right) \times H_{+}\left(L_{2}\right), \quad H_{+}\left(L_{1}, L_{2}\right)=H_{+}\left(L_{1}\right) \times H_{+}\left(L_{2}\right) \\
e \quad \operatorname{Ker}\left(L_{1}, L_{2}\right)=\operatorname{Ker} L_{1} \times \operatorname{Ker} L_{2} .
\end{gathered}
$$

Demonstração. De fato,

$$
\left(L_{1}, L_{2}\right)\left(H_{+}\left(L_{1}\right) \times H_{+}\left(L_{2}\right)\right)=L_{1}\left(H_{+}\left(L_{1}\right)\right) \times L_{2}\left(H_{+}\left(L_{2}\right)\right) \subseteq H_{+}\left(L_{1}\right) \times H_{+}\left(L_{2}\right),
$$

isto é, $H_{+}\left(L_{1}\right) \times H_{+}\left(L_{2}\right)$ é invariante por $\left(L_{1}, L_{2}\right)$.

Por outro lado, se $\left(x_{1}, x_{2}\right) \in H_{+}\left(L_{1}\right) \times H_{+}\left(L_{2}\right)$, com $x_{1}, x_{2} \neq 0$, de (3.1.1) temos

$$
\left\langle\left(L_{1}, L_{2}\right)\left(x_{1}, x_{2}\right),\left(x_{1}, x_{2}\right)\right\rangle=\left\langle\left(L_{1} x_{1}, L_{2} x_{2}\right),\left(x_{1}, x_{2}\right)\right\rangle=\left\langle L_{1} x_{1}, x_{1}\right\rangle+\left\langle L_{2} x_{2}, x_{2}\right\rangle>0 .
$$

Portanto, $\left(L_{1}, L_{2}\right)$ é definido positivo em $H_{+}\left(L_{1}\right) \times H_{+}\left(L_{2}\right)$.

Analogamente, $H_{-}\left(L_{1}\right) \times H_{-}\left(L_{2}\right)$ é invariante por $\left(L_{1}, L_{2}\right)$ e, além disso, $\left(L_{1}, L_{2}\right)$ é definido negativo em $H_{-}\left(L_{1}\right) \times H_{-}\left(L_{2}\right)$.

É fácil ver que

$$
\operatorname{Ker}\left(L_{1}, L_{2}\right)=\operatorname{Ker} L_{1} \times \operatorname{Ker} L_{2} .
$$

Consequentemente, o Teorema 3.3.19 implica que $H_{+}\left(L_{1}, L_{2}\right)=H_{+}\left(L_{1}\right) \times H_{+}\left(L_{2}\right)$ e $H_{-}\left(L_{1}, L_{2}\right)=H_{-}\left(L_{1}\right) \times H_{-}\left(L_{2}\right)$.

Lema 4.2.10. Se $P_{1}: H_{1} \rightarrow H_{1}$ e $P_{2}: H_{2} \rightarrow H_{2}$ são projeções ortogonais sobre $\operatorname{Im} P_{1}$ e $\operatorname{Im} P_{2}$, respectivamente, então $\left(P_{1}, P_{2}\right): H_{1} \times H_{2} \rightarrow \operatorname{Im} P_{1} \times \operatorname{Im} P_{2}$ é a projeção ortogonal sobre $\operatorname{Im} P_{1} \times \operatorname{Im} P_{2}$. 
Demonstração. É claro que $\operatorname{Im}\left(P_{1}, P_{2}\right)=\operatorname{Im} P_{1} \times \operatorname{Im} P_{2}$. Agora,

$$
\left(P_{1}, P_{2}\right)^{2}=\left(P_{1}, P_{2}\right)\left(P_{1}, P_{2}\right)=\left(P_{1} P_{1}, P_{2} P_{2}\right)=\left(P_{1}, P_{2}\right),
$$

isto é, $\left(P_{1}, P_{2}\right)$ é uma projeção. Por outro lado, dado que

$$
\left(P_{1}, P_{2}\right)^{*}=\left(P_{1}^{*}, P_{2}^{*}\right)=\left(P_{1}, P_{2}\right)
$$

$\left(P_{1}, P_{2}\right)$ é auto-adjunto. Consequentemente, a Proposição 3.2 .13 implica que $\left(P_{1}, P_{2}\right)$ é a projeção ortogonal sobre $\operatorname{Im} P_{1} \times \operatorname{Im} P_{2}$.

Observe que, se $\mathcal{J}_{1}$ e $\mathcal{J}_{2}$ são simetrias em $H_{1}$ e $H_{2}$, respectivamente, então $\left(\mathcal{J}_{1}, \mathcal{J}_{2}\right)$ é uma simetria em $H_{1} \times H_{2}$. De fato,

$$
\left(\mathcal{J}_{1}, \mathcal{J}_{2}\right)^{2}=\left(\mathcal{J}_{1}, \mathcal{J}_{2}\right)\left(\mathcal{J}_{1}, \mathcal{J}_{2}\right)=\left(\mathcal{J}_{1}^{2}, \mathcal{J}_{2}^{2}\right)=\left(I_{H_{1}}, I_{H_{2}}\right)=I_{H_{1} \times H_{2}}
$$

e, além disso, o Lema 4.2.9 implica que

$$
H_{+}\left(\mathcal{J}_{1}, \mathcal{J}_{2}\right)=H_{+}\left(\mathcal{J}_{1}\right) \times H_{+}\left(\mathcal{J}_{2}\right) \quad \text { e } \quad H_{-}\left(\mathcal{J}_{1}, \mathcal{J}_{2}\right)=H_{-}\left(\mathcal{J}_{1}\right) \times H_{-}\left(\mathcal{J}_{2}\right)
$$

Por outro lado, do Lema 2.3.8 temos que o produto direto de operadores compactos é compacto.

Proposição 4.2.11 (Assinatura do produto). Sejam $L_{1}=\mathcal{J}_{1}+K_{1} \in G L\left(H_{1}\right)$ e $L_{2}=$ $\mathcal{J}_{2}+K_{2} \in G L\left(H_{2}\right)$ perturbações compactas auto-adjuntas das simetrias $\mathcal{J}_{1} \in \Phi_{S}^{i}\left(H_{1}\right)$ $e \mathcal{J}_{2} \in \Phi_{S}^{i}\left(H_{2}\right)$, respectivamente. Então,

$$
\operatorname{sign}_{\left(\mathcal{J}_{1}, \mathcal{J}_{2}\right)}\left[\left(\mathcal{J}_{1}, \mathcal{J}_{2}\right)+\left(K_{1}, K_{2}\right)\right]=\operatorname{sign}_{\mathcal{J}_{1}}\left(\mathcal{J}_{1}+K_{1}\right)+\operatorname{sign}_{\mathcal{J}_{2}}\left(\mathcal{J}_{2}+K_{2}\right) .
$$

Demonstração. Sejam $\left(e_{1, i}^{ \pm}\right)_{i=1}^{\infty}$ e $\left(e_{2, i}^{ \pm}\right)_{i=1}^{\infty}$ bases de Hilbert para $H_{ \pm}\left(\mathcal{J}_{1}\right)$ e $H_{ \pm}\left(\mathcal{J}_{2}\right)$, respectivamente. Consideremos os subespaços

$$
H_{1, n}=\operatorname{span}\left\{e_{1, i}^{ \pm}: i=1, \ldots, n\right\} \quad \text { e } \quad H_{2, n}=\operatorname{span}\left\{e_{2, i}^{ \pm}: i=1, \ldots, n\right\} .
$$

Denotemos por $L_{1, n}$ e $L_{2, n}$ as $n$-ésimas aproximações de Galerkin de $L_{1}$ e $L_{2}$, respectivamente, com respeito as bases dadas acima. Então, $L_{1, n}=P_{1, n} L_{1}$ e $L_{2, n}=P_{2, n} L_{2}$, onde $P_{1, n}$ e $P_{1, n}$ são as projeções sobre $H_{1, n}$ e $H_{2, n}$, respectivamente. Se segue do lema anterior que $\left(P_{1, n}, P_{2, n}\right)$ é a projeção sobre $H_{1, n} \times H_{2, n}$. Logo,

$$
\left(L_{1, n}, L_{2, n}\right)=\left(P_{1, n} L_{1}, P_{2, n} L_{2}\right)=\left(P_{1, n}, P_{2, n}\right)\left(L_{1}, L_{2}\right),
$$

isto é, $\left(L_{1, n}, L_{2, n}\right)$ é a projeção de Galerkin do operador $\left(L_{1}, L_{2}\right)$.

Seja $n$ um inteiro positivo tal que

$$
\operatorname{sign}_{\mathcal{J}_{1}}\left(\mathcal{J}_{1}+K_{1}\right)=\operatorname{sign} L_{1, n} \quad \text { e } \quad \operatorname{sign}_{\mathcal{J}_{2}}\left(\mathcal{J}_{2}+K_{2}\right)=\operatorname{sign} L_{2, n} .
$$


Do Lema 4.1.5 temos

$$
\operatorname{sign}\left(L_{1, n}, L_{2, n}\right)=\operatorname{sign} L_{1, n}+\operatorname{sign} L_{2, n}=\operatorname{sign}_{\mathcal{J}_{1}}\left(\mathcal{J}_{1}+K_{1}\right)+\operatorname{sign}_{\mathcal{J}_{2}}\left(\mathcal{J}_{2}+K_{2}\right) .
$$

Assim,

$$
\begin{aligned}
\operatorname{sign}_{\left(\mathcal{J}_{1}, \mathcal{J}_{2}\right)}\left[\left(\mathcal{J}_{1}, \mathcal{J}_{2}\right)+\left(K_{1}, K_{2}\right)\right] & =\lim _{n \rightarrow \infty} \operatorname{sign}\left(L_{1, n}, L_{2, n}\right) \\
& =\operatorname{sign}_{\mathcal{J}_{1}}\left(\mathcal{J}_{1}+K_{1}\right)+\operatorname{sign}_{\mathcal{J}_{2}}\left(\mathcal{J}_{2}+K_{2}\right),
\end{aligned}
$$

o que prova a proposição.

\subsection{Funções de operadores}

Como falamos na introdução deste capítulo, o objetivo desta seção é mostrar que a aplicação que a cada operador auto-adjunto associa a projeção ortogonal sobre seu subespaço espectral negativo (positivo) é contínua. Esta propriedade será de grande utilidade, tanto como para a próxima seção, onde trataremos o índice de Morse relativo para pares de isomorfismos cuja diferença é compacta, que se definirá em função das projeções sobre os subespaços espectrais negativos, como também para o resto do trabalho.

Seja $L \in L(E)$, onde $E$ é un espaço de Banach complexo. Com base no Teorema da fórmula integral de Cauchy, nesta seção veremos que, se $f: \Delta \rightarrow \mathbb{C}$ é uma aplicação regular $^{2}$, onde $\Delta$ é um subconjunto aberto de $\mathbb{C}$ que contém $\sigma(L)$, podemos definir o operador $f(L) \in L(E)$ como

$$
f(L)=-\frac{1}{2 \pi i} \int_{\Gamma} f(\lambda)(L-\lambda I)^{-1} d \lambda,
$$

onde $\Gamma$ é um caminho (ou um número finito de caminhos que não se intersectam) fechado, simples, positivamente orientado, contido em $\Delta$ e que contém $\sigma(L)$ no seu interior. Para este fim, primeiro lembraremos alguns conceitos, tais como: caminhos retificáveis (fechados, simples ou positivamente orientados), aplicações regulares, integral de Riemann de aplicações regulares, entre outras. Além disso, apresentaremos algumas propriedades que possui a integral de Riemann de aplicações regulares ao longo de caminhos retificáveis, assim como também o Teorema da fórmula integral de Cauchy. A maioria destes resultados se podem ver, por exemplo, em [15] ou em [2]. Fazendo uso destas noções da análise complexa, será clara a fórmula acima que define o operador $f(L)$. Schechter em [25], §6.3 e §6.4, faz uma apresentação bem detalhada desta construção.

Finalmente, provaremos que, se $L \in L(H)$ é auto-adjunto, onde $H$ é um espaço de Hilbert complexo, e 0 não é ponto de acumulação de $\sigma(L)$, a projeção ortogonal sobre o

\footnotetext{
${ }^{2}$ Uma aplicação regular é também conhecida como holomorfa.
} 
subespaço espectral negativo de $L$ se pode expressar da forma $\chi(L)$, onde $\chi: \Delta \rightarrow \mathbb{C}$ é uma oportuna aplição regular e $\Delta$ é um subconjunto aberto dos complexos que contém $\sigma(L)$. No caso real, tomaremos a complexificação $\widehat{L}$ do operador $L$ e provaremos que a parte real do operador $\chi(\widehat{L})$ coincide com a projeção ortogonal sobre o subespaço espectral negativo de $L$. Com esta representação das projeções podemos mostrar que elas dependem continuamente dos operadores.

Nesta seção, se não se diz o contrario, $E$ denotará um espaço de Banach complexo e $\Delta$ um subconjunto aberto de $\mathbb{C}$.

Definição 4.3.1. Seja $\Gamma:[a, b] \rightarrow \mathbb{C}$ um caminho, isto é, uma aplicação contínua. Para qualquer partição de $[a, b]$, dada por $P=\left\{t_{0}, t_{1}, \ldots, t_{m}\right\}$, definimos

$$
\Lambda_{\Gamma}(P)=\sum_{k=1}^{m}\left\|\Gamma\left(t_{k}\right)-\Gamma\left(t_{k-1}\right)\right\| .
$$

Se

$$
\Lambda_{\Gamma}=\sup \left\{\Lambda_{\Gamma}(P): P \text { é uma partição de }[a, b]\right\}
$$

é finito, então dizemos que $\Gamma$ é retificável e seu comprimento é o supremo acima.

Definição 4.3.2. Seja $\Gamma:[a, b] \rightarrow \mathbb{C}$ um caminho.

Dizemos que $\Gamma$ é fechado se $\Gamma(a)=\Gamma(b)$. Neste caso o interior de $\Gamma$, denotado por $\stackrel{\circ}{\Gamma}$, é a região de $\mathbb{C}$ limitada por $\Gamma$.

Dizemos que $\Gamma$ é simples se $\Gamma\left(t_{1}\right) \neq \Gamma\left(t_{2}\right)$ para $t_{1}, t_{2} \in[a, b]$, com $t_{1} \neq t_{2}$ e pelo menos um dos dois é ponto interior de $[a, b]$. Se $t_{1}<t_{2}$, usaremos a notação $\Gamma\left(t_{1}\right)<\Gamma\left(t_{2}\right)$, sempre que $\Gamma\left(t_{1}\right)$ seja diferente de $\Gamma\left(t_{2}\right)$. Para simplificar, mesmo que seja impróprio, chamaremos de intervalo a imagem $\Gamma\left(\left[t_{1}, t_{2}\right]\right)$ e a denotaremos por $\left[\Gamma\left(t_{1}\right), \Gamma\left(t_{2}\right)\right]$. Além disso, escreveremos $\lambda \in \Gamma$ para denotar que $\lambda$ pertence à imagem de $\Gamma$.

Um caminho fechado $\Gamma$ é positivamente orientado se é percorrido no sentido antihorário, isto é, o seu interior fica à esquerda, ao se percorrer $\Gamma$. Por simplicidade, um caminho fechado, simples, retificável e positivamente orientado $\Gamma:[a, b] \rightarrow \mathbb{C}$ será chamado de curva fechada.

Definição 4.3.3. Dizemos que uma aplicação $f: \Delta \rightarrow E$ é regular (ou holomorfa) em $\lambda_{0} \in \Delta$, se existe em $E$ o limite

$$
\lim _{\lambda \rightarrow \lambda_{0}} \frac{f(\lambda)-f\left(\lambda_{0}\right)}{\lambda-\lambda_{0}}=f^{\prime}\left(\lambda_{0}\right) .
$$

Se $f$ é regular em cada ponto de $\Delta$, dizemos que $f$ é regular em $\Delta$ ou simplesmente que $f$ é regular.

Proposição 4.3.4. Seja $L \in L(E)$ dado. A aplicação $R: \rho(L) \rightarrow L(E)$ dada por $R(\lambda)=(L-\lambda I)^{-1}$, para $\lambda \in \rho(L)$, é regular. 
Demonstração. Sendo $\lambda \mapsto L-\lambda I$ contínua, do Lema 1.1.9 se segue que $R$ é contínua. Fixemos $\lambda_{0}$ em $\rho(L)$. Observe que, se $\lambda \in \rho(L)$, de (1.1.1) obtemos

$$
(L-\lambda I)^{-1}-\left(L-\lambda_{0} I\right)^{-1}=-(L-\lambda I)^{-1}\left(\lambda_{0} I-\lambda I\right)\left(L-\lambda_{0} I\right)^{-1},
$$

isto é,

$$
\frac{(L-\lambda I)^{-1}-\left(L-\lambda_{0} I\right)^{-1}}{\left(\lambda-\lambda_{0}\right)}=(L-\lambda I)^{-1}\left(L-\lambda_{0} I\right)^{-1} .
$$

Consequentemente,

$$
\lim _{\lambda \rightarrow \lambda_{0}} \frac{(L-\lambda I)^{-1}-\left(L-\lambda_{0} I\right)^{-1}}{\left(\lambda-\lambda_{0}\right)}=\left(L-\lambda_{0} I\right)^{-2},
$$

o que prova a proposição.

Lembremos agora a definição da integral de uma aplicação ao longo de um caminho contido no plano complexo. Consideremos um caminho retificável $\Gamma:[a, b] \rightarrow \Delta$. Uma partição da imagem de $\Gamma$ é um subconjunto $P=\left\{\lambda_{0}, \lambda_{1}, \lambda_{2}, \ldots, \lambda_{n}\right\} \subseteq \operatorname{Im}(\Gamma)$, onde $\lambda_{0}=\Gamma(a)$ e $\lambda_{n}=\Gamma(b)$, tal que

$$
\lambda_{0}<\lambda_{1}<\lambda_{2}<\ldots<\lambda_{n}
$$

Dizemos que uma partição $P^{\prime}$ da imagem de $\Gamma$ é mais fina que $P$ se $P \subseteq P^{\prime}$. A norma de uma partição $P$ da imagem de $\Gamma$ é definida por

$$
\|P\|=\max \left\{\left|\lambda_{i}-\lambda_{i-1}\right|: i=1, \ldots, n\right\} .
$$

É fácil ver que, se $P^{\prime}$ é mais fina que $P$, então $\left\|P^{\prime}\right\| \leq\|P\|$. Tomemos agora uma aplicação $f: \Delta \rightarrow E$ e um caminho retificável $\Gamma:[a, b] \rightarrow \Delta$. Para uma partição $P=\left\{\lambda_{0}, \lambda_{1}, \lambda_{2}, \ldots, \lambda_{n}\right\}$ da imagem de $\Gamma$, seja $E=\left\{\zeta_{1}, \zeta_{2}, \ldots, \zeta_{n}\right\}$, onde $\zeta_{i} \in\left[\lambda_{i-1}, \lambda_{i}\right]$, para $i=1,2, \ldots, n$. Neste caso diremos que $(P, E)$ é uma partição pontuada de $\Gamma$. Consideremos a soma

$$
S(P, E, f)=\sum_{i=1}^{n}\left(\lambda_{i}-\lambda_{i-1}\right) f\left(\zeta_{i}\right) .
$$

Definição 4.3.5. Dizemos que $f: \Delta \rightarrow E$ é integrável no caminho $\Gamma$ se existe um número $A$ com a seguinte propriedade: Para todo $\varepsilon>0$, existe uma partição pontuada $\left(P_{\varepsilon}, E_{\varepsilon}\right)$ da imagem de $\Gamma$ tal que para cada partição $P=\left\{\lambda_{0}, \lambda_{1}, \lambda_{2}, \ldots, \lambda_{n}\right\}$ mais fina que $P_{\varepsilon}$ e cada escolha dos pontos $E=\left\{\zeta_{1}, \zeta_{2}, \ldots, \zeta_{n}\right\}$, onde $\zeta_{i} \in\left[\lambda_{i-1}, \lambda_{i}\right]$, temos que

$$
|S(P, E, f)-A|<\varepsilon \text {. }
$$

Neste caso usaremos a notação

$$
\lim _{\|P\| \rightarrow 0} S(P, E, f)=A
$$


O número $A$ é chamado de integral de $f$ no caminho $\Gamma$ e será denotado por

$$
\int_{\Gamma} f(\lambda) d \lambda
$$

No caso das funções contínuas reais de variável real, o análogo da noção de integral acima é equivalente à definição clássica de integral de Riemann, dada com as somas superiores e inferiores.

Teorema 4.3.6. Se $f: \Delta \rightarrow E$ é contínua, então ela é integrável em qualquer caminho retificável $\Gamma$ contido em $\Delta$.

Podemos encontrar uma prova do teorema anterior, por exemplo, em [15], Cápitulo 3 , 9 . Como consequência obtemos que, se $f$ é contínua, para qualquer sequência de partições pontuadas $\left(P_{n}, E_{n}\right)_{n=1}^{\infty}$ de $\Gamma$ tal que $\lim _{n \rightarrow \infty}\left\|P_{n}\right\|=0$, então

$$
\int_{\Gamma} f(\lambda) d \lambda=\lim _{n \rightarrow \infty} S\left(P_{n}, E_{n}, f\right) .
$$

A seguir apresentaremos alguns resultados clássicos da teoria das funções complexas que serão usados nesta seção. Os dois seguintes resultados são os bem conhecidos Teorema integral de Cauchy e a Fórmula integral de Cauchy, cujas provas se podem encontrar, por exemplo, em [15], pág. 57, Teorema 2, e pág. 61, respectivamente.

Teorema 4.3.7 (Teorema integral de Cauchy). Sejam $f: \Delta \rightarrow E$ uma aplicação regular e $\Gamma_{0}$ uma curva fechada contida em $\Delta$. Suponhamos que $\Gamma_{1}, \Gamma_{2}, \ldots, \Gamma_{m}$ sejam curvas fechadas contidas no interior de $\Gamma_{0}$ e que cada uma delas esteja contida no exterior de todas as outras. Isto é, para cada $i=1,2, \ldots, m, \Gamma_{i}$ está contida no exterior de cada uma das curvas $\Gamma_{1}, \Gamma_{2}, \ldots, \Gamma_{i-1}, \Gamma_{i+1}, \ldots, \Gamma_{m}$. Então,

$$
\int_{\Gamma_{0}} f(\lambda) d \lambda=\int_{\Gamma_{1}} f(\lambda) d \lambda+\int_{\Gamma_{2}} f(\lambda) d \lambda+\ldots+\int_{\Gamma_{m}} f(\lambda) d \lambda,
$$

sempre que todas as curvas fechadas e as regiões anulares entre $\Gamma_{0}$ e os $\Gamma_{i}$, para $i=$ $1,2, \ldots, m$, estejam contidas inteiramente em $\Delta$.

Teorema 4.3.8 (Fórmula integral de Cauchy). Seja $f: \Delta \rightarrow E$ uma aplicação regular. Se $\Gamma$ é uma curva fechada, então a fórmula

$$
f(\zeta)=-\frac{1}{2 \pi i} \int_{\Gamma} \frac{f(\lambda)}{\zeta-\lambda} d \lambda
$$

é valida para todo ponto $\zeta$ no interior de $\Gamma$, sempre que $\Gamma$ e seu interior estejam contidos inteiramente em $\Delta$. 
Uma característica das aplicações regulares com valores em $\mathbb{C}$ é que todas suas derivadas de ordem superior existem e, além disso, elas se podem expressar localmente como uma série de potências, como mostra o seguinte teorema. Uma prova deste teorema se pode ver em [15], pág. 79, Teorema 1.

Teorema 4.3.9. Sejam $f: \Delta \rightarrow \mathbb{C}$ uma aplicação regular e $\lambda_{0} \in \Delta$. Então, existe uma, e somente uma, série de potências da forma

$$
\sum_{k=0}^{\infty} a_{k}\left(\lambda-\lambda_{0}\right)^{k}
$$

a qual converge ao menos na maior bola contida em $\Delta$ com centro em $\lambda_{0}$ e representa a função $f(\lambda)$ em tal bola. Além disso,

$$
a_{k}=\frac{1}{k !} f^{(k)}\left(\lambda_{0}\right) .
$$

Fixemos um operador $L \in L(E)$. É sabido que, para um polinômio $p: \mathbb{C} \rightarrow \mathbb{C}$ dado por $p(\lambda)=\sum_{k=0}^{n} a_{k} \lambda^{k}$, podemos definir o operador $p(L)$ como

$$
p(L)=\sum_{k=0}^{n} a_{k} L^{k}, \quad \text { onde } L^{0}=I .
$$

Vamos agora abordar a definição de $f(L)$, onde $f: \Delta \rightarrow \mathbb{C}$ é uma aplicação regular tal que $\sigma(L) \subseteq \Delta$, como foi introduzida na fórmula (4.3.1). Primeiro mostraremos o caso em que $\Delta$ é uma bola aberta em $\mathbb{C}$ e depois o caso geral. Antes de apresentar esta definição, primeiro vejamos os seguintes resultados, cujas provas se podem ver, por exemplo, em [7], pág. 197, Proposição 3.8 e [25], pág. 150, Lema 6.28, respectivamente.

Lema 4.3.10. Se $L \in L(E)$, então o limite

$$
\lim _{k \rightarrow \infty}\left\|L^{k}\right\|^{1 / k}
$$

existe e coincide com

$$
\max _{\lambda \in \sigma(L)}|\lambda|
$$

O número $r(L)=\max _{\lambda \in \sigma(L)}|\lambda|$ no lema anterior é chamado de raio espectral de $L$.

Lema 4.3.11. Se $A$ é um subconjunto compacto de $\Delta$, existe um conjunto aberto limitado $\omega$ tal que:

i. $A \subseteq \omega$,

ii. $\bar{\omega} \subseteq \Delta$, 
iii. a fronteira de $\omega$ consiste de um número finito de curvas fechadas $\Gamma_{1}, \Gamma_{2}, \ldots, \Gamma_{n}$ as quais não se intersectam.

Denotaremos por $\partial \omega$ a fronteira de $\omega$. Tomando $A=\sigma(L)$ no lema anterior, obtemos que

$$
\sigma(L) \subseteq \omega=\bigcup_{i=1}^{n} \stackrel{\circ}{\Gamma}_{i} \subseteq \bigcup_{i=1}^{n} \stackrel{\circ}{\Gamma}_{i} \subseteq \Delta .
$$

Sejam $L \in L(E)$ e $f: B(0, \varepsilon) \rightarrow \mathbb{C}$ uma aplicação regular tal que $\sigma(L) \subseteq B(0, \varepsilon)$. Do Teorema 4.3.9 se segue que

$$
f(\lambda)=\sum_{k=0}^{\infty} a_{k} \lambda^{k} \quad \text { para todo } \lambda \in B(0, \varepsilon) .
$$

Definimos, analogamente, a série formal de potências de $L$

$$
\sum_{k=0}^{\infty} a_{k} L^{k}
$$

e provemos que converge em $L(E)$. De fato, observe que

$$
r(L)=\max _{\lambda \in \sigma(L)}|\lambda|<\varepsilon,
$$

pois $\sigma(L) \subseteq B(0, \varepsilon)$ e $\sigma(L)$ é compacto. Assim,

$$
\sigma(L) \subseteq B(0, \delta), \quad \text { onde } \delta=(r(L)+\varepsilon) / 2 .
$$

Dado que

$$
\lim _{k \rightarrow \infty}\left\|L^{k}\right\|^{1 / k}=r(L),
$$

existe $N \in \mathbb{N}$ tal que $\left\|L^{k}\right\|^{1 / k}<\delta<\varepsilon$ para $k \geq N$. Como consequência do Teorema 4.3.9, temos que a série $\sum_{k=0}^{\infty}\left|a_{k}\right| \delta^{k}$ é convergente em $\mathbb{C}$. Por outro lado,

$$
\sum_{k=N}^{\infty}\left\|a_{k} L^{k}\right\| \leq \sum_{k=N}^{\infty}\left|a_{k}\right|\|L\|^{k} \leq \sum_{k=N}^{\infty}\left|a_{k}\right| \delta^{k} \leq \sum_{k=0}^{\infty}\left|a_{k}\right| \delta^{k} .
$$

Este fato implica que a série $\sum_{k=0}^{\infty}\left\|a_{k} L^{k}\right\|$ é convergente. Portanto, dado que $L(E)$ é um espaço de Banach, pelo Teorema 1.1.2 concluímos que $\sum_{k=0}^{\infty} a_{k} L^{k}$ converge em $L(E)$. Consequentemente, o operador

$$
f(L)=\sum_{k=0}^{\infty} a_{k} L^{k} \in L(E)
$$


é bem definido. Como resultado análogo ao Teorema 4.3.8 (fórmula integral de Cauchy), provaremos que $f(L)$ coincide com a integral

$$
-\frac{1}{2 \pi i} \int_{\Gamma} f(\lambda)(L-\lambda I)^{-1} d \lambda,
$$

onde $\Gamma$ é uma curva fechada contida em $B(0, \varepsilon)$ que contém no seu interior o espectro de $L$. Para este fim, vejamos o seguinte teorema, cuja demostração pode ser encontrada, por exemplo, em [25], pág. 136, Teorema 6.12.

Teorema 4.3.12. Sejam $L$ um operador em $L(E)$ e $\Gamma:[a, b] \rightarrow \mathbb{C}$ um curva fechada tal que $\sigma(L) \subseteq \stackrel{\Gamma}{\Gamma}$. Então, para cada inteiro positivo $k$, temos

$$
L^{k}=-\frac{1}{2 \pi i} \int_{\Gamma} \lambda^{k}(L-\lambda I)^{-1} d \lambda .
$$

Sejam $L \in L(E)$ e $f: B(0, \varepsilon) \rightarrow \mathbb{C}$ uma aplicação regular tal que $\sigma(L) \subseteq B(0, \varepsilon)$.

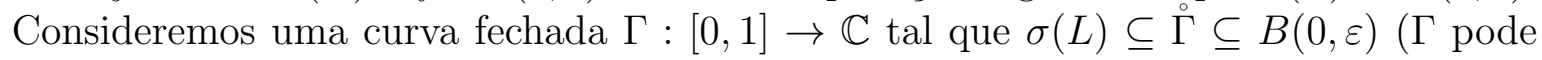
ser, por exemplo, a curva dada por $\Gamma(t)=\delta e^{2 \pi i t}$, isto é, a circunferência de raio $\delta$, onde $\delta=(r(L)+\varepsilon) / 2$ como em (4.3.5)). Então, de (4.3.6) e do teorema anterior, temos

$$
\begin{aligned}
f(L) & =\sum_{k=0}^{\infty} a_{k} L^{k}=-\frac{1}{2 \pi i} \sum_{k=0}^{\infty} a_{k} \int_{\Gamma} \lambda^{k}(L-\lambda I)^{-1} d \lambda \\
& =-\frac{1}{2 \pi i} \int_{\Gamma} \sum_{k=0}^{\infty} a_{k} \lambda^{k}(L-\lambda I)^{-1} d \lambda=-\frac{1}{2 \pi i} \int_{\Gamma} f(\lambda)(L-\lambda I)^{-1} d \lambda,
\end{aligned}
$$

o que prova que a fórmula (4.3.1) é válida para o caso $\Delta=B(0, \varepsilon)$.

De forma análoga à expressão acima podemos definir o operador $f(L)$ para qualquer aplicação regular $f: \Delta \rightarrow \mathbb{C}$, onde $\Delta \subseteq \mathbb{C}$ é um subconjunto aberto (não necessariamente uma bola aberta em $\mathbb{C}$ ) que contém o espectro de $L$, como veremos na seguinte definição.

Definição 4.3.13. Suponhamos que $L \in L(E)$ e que $f: \Delta \rightarrow \mathbb{C}$ seja uma aplicação regular tal que $\sigma(L) \subseteq \Delta$. Seja $\omega \subseteq \mathbb{C}$ um aberto tal que sua fronteira consista de um número finito de curvas fechadas $\Gamma_{1}, \ldots, \Gamma_{n} \mathrm{e}$

$$
\sigma(L) \subseteq \omega=\bigcup_{i=1}^{n} \stackrel{\circ}{\Gamma}_{i} \subseteq \overline{\bigcup_{i=1}^{n} \stackrel{\circ}{\Gamma}_{i}} \subseteq \Delta .
$$

O operador $f(L)$ é definido como

$$
f(L)=-\frac{1}{2 \pi i} \int_{\partial \omega} f(\lambda)(L-\lambda I)^{-1} d \lambda .
$$


A existência da integral (4.3.7) se deve a que $\partial \omega$ consiste de um número finito de curvas fechadas e que a aplicação

$$
\lambda \mapsto f(\lambda)(L-\lambda I)^{-1}
$$

é regular em $\partial \omega$ e portanto contínua.

$\mathrm{Na}$ definição anterior não foi exigida a conexidade de $\Delta$ ou de $\sigma(L)$. Como consequência do Lema 4.3.11 temos que, se $\sigma(L)$ é conexo, existe uma curva fechada $\Gamma$ contida em $\Delta$ e que contém no seu interior o espectro de $L$. Assim,

$$
f(L)=-\frac{1}{2 \pi i} \int_{\Gamma} f(\lambda)(L-\lambda I)^{-1} d \lambda .
$$

Suponhamos agora que

$$
\sigma(L)=\sigma_{1} \cup \sigma_{2},
$$

onde $\sigma_{1}$ e $\sigma_{2}$ são subconjuntos de $\mathbb{C}$ separados, isto é, existem dois conjuntos abertos disjuntos $\Delta_{1}$ e $\Delta_{2}$ tais que $\sigma_{1} \subseteq \Delta_{1}$ e $\sigma_{2} \subseteq \Delta_{2}$. Não é difícil provar que $\sigma_{1}$ e $\sigma_{2}$ são subconjuntos compactos de $\mathbb{C}$. Seja $f$ uma aplicação complexa e regular em $\Delta_{0}=$ $\Delta_{1} \cup \Delta_{2}$. Se $\Gamma_{1}$ e $\Gamma_{2}$ são duas curvas fechadas tais que

$$
\sigma_{1} \subseteq \stackrel{\circ}{\Gamma}_{1} \subseteq \Delta_{1} \quad \text { e } \quad \sigma_{2} \subseteq \stackrel{\circ}{\Gamma}_{2} \subseteq \Delta_{2}
$$

então, por definição,

$$
f(L)=-\frac{1}{2 \pi i} \int_{\Gamma_{1} \cup \Gamma_{2}} f(\lambda)(L-\lambda I)^{-1} d \lambda
$$

isto é,

$$
f(L)=-\left(\frac{1}{2 \pi i} \int_{\Gamma_{1}} f(\lambda)(L-\lambda I)^{-1} d \lambda+\frac{1}{2 \pi i} \int_{\Gamma_{2}} f(\lambda)(L-\lambda I)^{-1} d \lambda\right) .
$$

Por outro lado, além das condições do caso anterior, suponhamos também que $f$ seja regular em uma região conexa $\Delta$ que contém $\Delta_{0}$ e que $\Gamma_{0}$ seja uma curva fechada contida em $\Delta$ e que contém no seu interior as curvas $\Gamma_{1}$ e $\Gamma_{2}$. Se segue do Teorema 4.3.7 (Teorema integral de Cauchy), para o caso de integrais de aplicações definidas em $L(E)$, que

$$
\frac{1}{2 \pi i} \int_{\Gamma_{0}} f(\lambda)(L-\lambda I)^{-1} d \lambda=\frac{1}{2 \pi i} \int_{\Gamma_{1}} f(\lambda)(L-\lambda I)^{-1} d \lambda+\frac{1}{2 \pi i} \int_{\Gamma_{2}} f(\lambda)(L-\lambda I)^{-1} d \lambda .
$$

Este fato mostra que, se $\Delta$ é uma bola aberta com centro em 0 , a definição do operador $f(L)$ dada em (4.3.7) coincide com (4.3.6).

No caso em que $\sigma(L)$ e o domínio de $f$ possuam um número finito de componentes conexas, obtemos uma expressão do operador $f(L)$ análoga à dada acima. 
Observação 4.3.14. Suponhamos que temos $f(L)$ como em (4.3.7). É fácil ver que $L$ comuta com $(L-\lambda I)^{-1}$ para $\lambda \in \rho(L)$. Assim,

$$
\begin{aligned}
L f(L) & =L\left(-\frac{1}{2 \pi i} \int_{\partial \omega} f(\lambda)(L-\lambda I)^{-1} d \lambda\right)=-\frac{1}{2 \pi i} \int_{\partial \omega} f(\lambda) L(L-\lambda I)^{-1} d \lambda \\
& =-\frac{1}{2 \pi i} \int_{\partial \omega} f(\lambda)(L-\lambda I)^{-1} L d \lambda=\left(-\frac{1}{2 \pi i} \int_{\partial \omega} f(\lambda)(L-\lambda I)^{-1} d \lambda\right) L \\
& =f(L) L .
\end{aligned}
$$

Logo,

$$
L f(L)=f(L) L
$$

Outras duas propriedades importantes que possui o operador $f(L)$ são apresentadas nos dois seguintes lemas, cujas provas se podem encontrar, por exemplo, em [25], pág. 138, Lema 6.15 e pág. 139, Teorema 6.17, respectivamente.

Lema 4.3.15. Seja $L \in L(E)$ fixado. Suponhamos que $f: \Delta \rightarrow \mathbb{C}$ e $g: \Delta \rightarrow \mathbb{C}$ sejam duas aplicações regulares e que $\sigma(L) \subseteq \Delta$. Se $h: \Delta \rightarrow \mathbb{C}$ é definida como $h(\lambda)=f(\lambda) g(\lambda)$, então

$$
h(L)=f(L) g(L) .
$$

Lema 4.3.16. Seja $L \in L(E)$ fixado. Se $f: \Delta \rightarrow \mathbb{C}$ é regular em uma vizinhança de $\sigma(L)$, então

$$
\sigma(f(L))=f(\sigma(L))
$$

isto é, $\lambda \in \sigma(f(L))$ se, e somente se, $\lambda=f(\zeta)$ para algum $\zeta \in \sigma(L)$.

Com falamos na introdução desta seção, provaremos que a projeção ortogonal sobre o subespaço espectral negativo de um operador auto-adjunto $L$, definido em um espaço de Hilbert complexo $H$, se pode expressar da forma $\chi(L)$, onde $\chi$ é uma aplicação regular em um subconjunto aberto que contém o espectro de $L$. Mais geralmente, veremos que, se $\sigma(L)=\sigma_{1} \cup \sigma_{2}$, onde $\sigma_{1}$ e $\sigma_{2}$ são dois subconjuntos separados de $\sigma(L)$, então:

i. existe um subespaço fechado $H_{1} \subseteq H$ tal que $H_{1}$ e $H_{1}^{\perp}$ são invariantes por $L$,

ii. os espectros das restrições $\left.L\right|_{H_{1}}: H_{1} \rightarrow H_{1}$ e $\left.L\right|_{H_{1}^{\perp}}: H_{1}^{\perp} \rightarrow H_{1}^{\perp}$ do operador $L$ são $\sigma_{1}$ e $\sigma_{2}$, respectivamente, e

iii. a projeção ortogonal sobre $H_{1}$ pode ser expressada em função de $L$.

De fato, sejam $H$ um espaço de Hilbert complexo e $L \in L(H)$ um operador autoadjunto. Neste caso $\sigma(L)$ é um subconjunto dos números reais. Suponhamos que

$$
\sigma(L)=\sigma_{1} \cup \sigma_{2}
$$


e que exista uma bola aberta $B(a, \varepsilon)$ com centro em $a \in \mathbb{R}$ e raio $\varepsilon>0$ tal que

$$
\sigma_{1} \subseteq B(a, \varepsilon) \quad \text { e } \quad \sigma_{2} \subseteq \mathbb{C} \backslash \overline{B(a, \varepsilon)} .
$$

Assim, como em (4.3.5), existe uma bola aberta $B(a, \delta)$, com $0<\delta<\varepsilon$, tal que $\sigma_{1} \subseteq B(a, \delta)$. Tomemos $\Gamma:[0,1] \rightarrow \mathbb{C}$ definido por

$$
\Gamma(t)=a+\delta e^{2 \pi i t},
$$

isto é, $\Gamma$ é uma parametrização (orientada positivamente) da circunferência de centro em $a$ e raio $\delta$. Daí,

$$
\sigma_{1} \subseteq \stackrel{\circ}{\Gamma} \subseteq B(a, \varepsilon)
$$

Por outro lado, observe que

$$
\sigma_{2}=\sigma_{2}^{\prime} \cup \sigma_{2}^{\prime \prime},
$$

onde $\sigma_{2}^{\prime}=\sigma_{2} \cap\left(-\infty, \min \sigma_{1}\right)$ e $\sigma_{2}^{\prime \prime}=\sigma_{2} \cap\left(\max \sigma_{1},+\infty\right)\left(\sigma_{2}^{\prime}\right.$ ou $\sigma_{2}^{\prime \prime}$ podem ser vazios, porém, obviamente não ambos). Como vimos acima, existem dois curvas fechadas $\Gamma^{\prime} \mathrm{e}$ $\Gamma^{\prime \prime}$ contidas em $\mathbb{C} \backslash \overline{B(a, \varepsilon)}$ tais que

$$
\sigma_{2}^{\prime} \subseteq \stackrel{\circ}{ }^{\prime} \subseteq \mathbb{C} \backslash \overline{B(a, \varepsilon)} \quad \text { e } \quad \sigma_{2}^{\prime \prime} \subseteq \Gamma^{\prime \prime} \subseteq \mathbb{C} \backslash \overline{B(a, \varepsilon)} .
$$

A aplicação $\chi$ dada por

$$
\chi(\lambda)= \begin{cases}1 & \text { se } \lambda \in B(a, \varepsilon) \\ 0 & \text { se } \lambda \in \mathbb{C} \backslash \overline{B(a, \varepsilon)}\end{cases}
$$

é regular no conjunto aberto $\Delta=B(a, \varepsilon) \cup \mathbb{C} \backslash \overline{B(a, \varepsilon)}$. Assim,

$$
\chi(L)=-\frac{1}{2 \pi i} \int_{\Gamma \cup \Gamma^{\prime} \cup \Gamma^{\prime \prime}}(L-\lambda I)^{-1} d \lambda .
$$

De (4.3.8) temos

$$
\chi(L)=-\frac{1}{2 \pi i} \int_{\Gamma}(L-\lambda I)^{-1} d \lambda .
$$

Proposição 4.3.17. O operador $\chi(L)$ é uma projeção ortogonal.

Demonstração. Denotemos por $P$ o operador $\chi(L)$. Dado que $\chi(\lambda) \chi(\lambda)=\chi(\lambda)$ para todo $\lambda \in \Delta$, do Lema 4.3.15 temos

$$
P^{2}=\chi(L) \chi(L)=\chi(L)=P .
$$

Vejamos que $P$ é auto-adjunto. De fato, não é difícil provar que, para qualquer operador $T \in L(H)$,

$$
\rho\left(T^{*}\right)=\{\bar{\lambda}: \lambda \in \rho(T)\},
$$


onde $\bar{\lambda}$ denota o conjugado de $\lambda \in \mathbb{C}$. Logo, dado que $L$ é auto-adjunto e $\Gamma \subseteq \rho(L)$, se $\lambda \in \Gamma$, então $L-\bar{\lambda} I$ é inversível. Dada uma partição $A=\left\{\lambda_{0}, \lambda_{1}, \ldots, \lambda_{n}\right\}$ da imagem de $\Gamma$, onde $\Gamma(0)=\lambda_{0}<\lambda_{1}<\lambda_{2}<\ldots<\lambda_{n}=\lambda_{0}$, tomemos $E_{A}=\left\{\lambda_{1}, \lambda_{2}, \ldots, \lambda_{n}\right\}$. Sejam $R$ a aplicação resolvente do operador $L$ e $x, y \in H$. Logo, como na fórmula (4.3.2), temos

$$
\begin{aligned}
\left\langle S\left(A, E_{A},-R / 2 \pi i\right) x, y\right\rangle & =\left\langle-\frac{1}{2 \pi i} \sum_{k=1}^{n}\left(\lambda_{k}-\lambda_{k-1}\right)\left(L-\lambda_{k} I\right)^{-1} x, y\right\rangle \\
& =\left\langle x, \frac{1}{2 \pi i} \sum_{k=1}^{n}\left(\bar{\lambda}_{k}-\bar{\lambda}_{k-1}\right)\left(L^{*}-\bar{\lambda}_{k} I\right)^{-1} y\right\rangle \\
& =\left\langle x, \frac{1}{2 \pi i} \sum_{k=1}^{n}\left(\bar{\lambda}_{k}-\bar{\lambda}_{k-1}\right)\left(L-\bar{\lambda}_{k} I\right)^{-1} y\right\rangle .
\end{aligned}
$$

A definição de $\Gamma$ (ver (4.3.10)) implica que $\bar{\lambda}_{k} \in \Gamma$ e que $\bar{\lambda}_{n}<\bar{\lambda}_{n-1}<\ldots<\bar{\lambda}_{0}=\bar{\lambda}_{n}$. Para $k=0,1, \ldots, n$, seja $\xi_{k}=\bar{\lambda}_{n-k}$. Assim, $\xi_{0}<\xi_{1}<\ldots<\xi_{n}=\xi_{0}$, isto é, $B=$ $\left\{\xi_{0}, \xi_{1}, \ldots, \xi_{n}\right\}$ é uma partição da imagem de $\Gamma$. Consideremos $E_{B}=\left\{\xi_{0}, \xi_{2}, \ldots, \xi_{n-1}\right\}$. Consequentemente,

$$
\begin{aligned}
\left\langle x, \frac{1}{2 \pi i} \sum_{k=1}^{n}\left(\bar{\lambda}_{k}-\bar{\lambda}_{k-1}\right)\left(L-\bar{\lambda}_{k} I\right)^{-1} y\right\rangle & =\left\langle x, \frac{1}{2 \pi i} \sum_{k=1}^{n}\left(\xi_{n-k}-\xi_{n-k+1}\right)\left(L-\xi_{n-k} I\right)^{-1} y\right\rangle \\
& =\left\langle x,-\frac{1}{2 \pi i} \sum_{k=1}^{n}\left(\xi_{n-k+1}-\xi_{n-k}\right)\left(L-\xi_{n-k} I\right)^{-1} y\right\rangle \\
& =\left\langle x,-\frac{1}{2 \pi i} \sum_{j=1}^{n}\left(\xi_{j}-\xi_{j-1}\right)\left(L-\xi_{j-1} I\right)^{-1} y\right\rangle \\
& =\left\langle x, S\left(B, E_{B},-R / 2 \pi i\right) y\right\rangle .
\end{aligned}
$$

Portanto,

$$
\left\langle S\left(A, E_{A},-R / 2 \pi i\right) x, y\right\rangle=\left\langle x, S\left(B, E_{B},-R / 2 \pi i\right) y\right\rangle \quad \text { para todo } x, y \in H .
$$

Da definição de $P$, obtemos que

$$
\lim _{\|A\| \rightarrow 0} S\left(A, E_{A},-R / 2 \pi i\right)=P=\lim _{\|B\| \rightarrow 0} S\left(B, E_{B},-R / 2 \pi i\right) .
$$

Portanto, de (4.3.12) e da continuidade do produto interno,

$$
\langle P x, y\rangle=\langle x, P y\rangle \quad \text { para todo } x, y \in H,
$$

isto é, $P=P^{*}$, o que prova a proposição. 
Da proposição anterior se segue que $H=\operatorname{Im} \chi(L) \oplus \operatorname{Ker} \chi(L)$ e que, além disso, $\operatorname{Im} \chi(L)$ e $\operatorname{Ker} \chi(L)$ são subespaços ortogonais de $H$. Por outro lado, de (4.3.9) temos

$$
L \chi(L)=\chi(L) L .
$$

Portanto, $\operatorname{Im} \chi(L)$ e Ker $\chi(L)$ são invariantes por $L$. Para a seguinte proposição denotaremos por $L_{1}$ e $L_{2}$ as restrições

$$
\left.L\right|_{\operatorname{Im} \chi(L)}: \operatorname{Im} \chi(L) \rightarrow \operatorname{Im} \chi(L) \quad \text { e }\left.\quad L\right|_{\operatorname{Ker} \chi(L)}: \operatorname{Ker} \chi(L) \rightarrow \operatorname{Ker} \chi(L)
$$

do operador $L$, respectivamente.

Proposição 4.3.18. Os espectros das restrições $L_{1}$ e $L_{2}$ são $\sigma_{1}$ e $\sigma_{2}$, respectivamente.

Demonstração. Como na proposição anterior, seja $P=\chi(L)$. A Proposição 3.3 .7 prova que

$$
\sigma(L)=\sigma\left(L_{1}\right) \cup \sigma\left(L_{2}\right) .
$$

Provemos que $\sigma\left(L_{1}\right)=\sigma_{1}$. De fato, seja $\xi \in \mathbb{C}$ um ponto que não pertença a $\sigma_{1}$. Denotemos por $\Delta_{1}=B(a, \delta)$ e $\Delta_{2}=\mathbb{C} \backslash \overline{B(a, \varepsilon)}$. Seja $\Delta_{1}^{\prime} \subseteq \Delta_{1}$ subconjunto aberto tal que $\sigma_{1} \subseteq \Delta_{1}^{\prime}$ e $\xi \notin \Delta_{1}^{\prime}$. Assim, $\Delta_{1}^{\prime} \cup \Delta_{2}$ é uma vizinhança aberta de $\sigma(L)$. Tomemos

$$
g(\lambda)= \begin{cases}\chi(\lambda) /(\xi-\lambda) & \text { se } \lambda \in \Delta_{1}^{\prime} \\ 0 & \text { se } \lambda \in \Delta_{2}\end{cases}
$$

Então, $g$ é regular em $\Delta_{1}^{\prime} \cup \Delta_{2}$. Observe que $\chi(\lambda) g(\lambda)=0=g(\lambda)$ para $\lambda \in \Delta_{2} \mathrm{e}$

$$
\chi(\lambda) g(\lambda)=\chi(\lambda) \chi(\lambda) /(\xi-\lambda)=\chi(\lambda) /(\xi-\lambda)=g(\lambda) \quad \text { para } \lambda \in \Delta_{1}^{\prime} .
$$

isto é, $\chi(\lambda) g(\lambda)=g(\lambda)$ para todo $\lambda \in \Delta_{1}^{\prime} \cup \Delta_{2}$. Logo,

$$
P g(L)=\chi(L) g(L)=g(L) .
$$

Consequentemente, $\operatorname{Im} P$ é invariante por $g(L)$. Consideremos a restrição $\left.g(L)\right|_{\operatorname{Im} P}$ : $\operatorname{Im} P \rightarrow \operatorname{Im} P$ do operador $g(L)$. Como $\chi(\lambda)=g(\lambda)(\xi-\lambda)$ para todo $\lambda \in \Delta_{1}^{\prime} \cup \Delta_{2}$, então

$$
P=\chi(L)=g(L)(\xi I-L) .
$$

Assim, $\left.g(L)\right|_{\operatorname{Im} P}: \operatorname{Im} P \rightarrow \operatorname{Im} P$ é a inversa da restrição $\left.(\xi I-L)\right|_{\operatorname{Im} P}: \operatorname{Im} P \rightarrow \operatorname{Im} P$, pois $\left.P\right|_{\operatorname{Im} P}$ é a identidade de $\operatorname{Im} P$. Este fato prova que $\left.\xi I\right|_{\operatorname{Im} P}-L_{1}$ é inversível, isto é, $\xi \in \rho\left(L_{1}\right)$. Em conclusão, $\sigma\left(L_{1}\right) \subseteq \sigma_{1}$.

Analogamente podemos provar que $\sigma\left(L_{2}\right) \subseteq \sigma_{2}$.

Por outro lado, como

$$
\sigma(L)=\sigma_{1} \cup \sigma_{2}=\sigma\left(L_{1}\right) \cup \sigma\left(L_{2}\right),
$$

$\sigma_{1}$ e $\sigma_{2}$ são disjuntos e $\sigma\left(L_{i}\right) \subseteq \sigma_{i}$ para $i=1,2$, temos que $\sigma\left(L_{i}\right)=\sigma_{i}$. 
Definição 4.3.19. O operador $\chi(L)$, definido acima, é chamado de projeção sobre o subespaço espectral de $L$ correspondente a $\sigma_{1}$.

Demonstremos agora que, se $\sigma_{1}=\sigma^{-}(L)$, para um operador auto-adjunto $L \in$ $L(H)$, e se 0 não é ponto de acumulação do espectro de $L$, o operador $\chi(L)$ em (4.3.11) é a projeção ortogonal sobre o subespaço espectral negativo de $L$. De fato, seja $L \in L(H)$ auto-adjunto. Primeiro provemos que

$$
\lambda_{0}=\max _{\lambda \in \sigma(L)} \lambda=M=\sup _{\|x\|=1}\langle L x, x\rangle .
$$

Da Proposição 3.3.9 se segue $\lambda_{0} \leq M$. Suponhamos por contradição que $M>\lambda_{0}$. Assim, $L-M I$ é inversível e não positivo. Consequentemente, a Observação 3.3.15 implica que $L-M I$ é definido negativo. Pela Observação 3.4.6, temos que existe $l<0$ tal que

$$
\sup _{\|x\|=1}\langle(L-M I) x, x\rangle \leq l .
$$

Porém,

$$
\sup _{\|x\|=1}\langle(L-M I) x, x\rangle=\sup _{\|x\|=1}(\langle L x, x\rangle-M\langle x, x\rangle)=\sup _{\|x\|=1}\langle L x, x\rangle-M=0,
$$

contradizendo (4.3.15). Portanto, $M=\lambda_{0}$.

Analogamente podemos provar que

$$
\min _{\lambda \in \sigma(L)} \lambda=\inf _{\|x\|=1}\langle L x, x\rangle .
$$

Suponhamos ainda que $L \in L(H)$ seja um operador auto-adjunto e que 0 não seja ponto de acumulação de $\sigma(L)$. É claro que existem duas bolas abertas disjuntas $B_{1}$, e $B_{2}$ em $\mathbb{C}$ tais que

$$
\sigma^{-}(L) \subseteq B_{1}, \quad\{0\} \cup \sigma^{+}(L) \subseteq B_{2} .
$$

Como em (4.3.11), podemos definir a projeção $P_{-}$sobre o subespaço espectral de $L$ correspondente a $\sigma^{-}(L)$ como um operador $\chi(L)$. Logo,

$$
H=\operatorname{Im} P_{-} \oplus \operatorname{Ker} P_{-}
$$

onde a soma é ortogonal.

Agora, o espectro da restrição $\left.L\right|_{\operatorname{Im} P_{-}}: \operatorname{Im} P_{-} \rightarrow \operatorname{Im} P_{-}$, que é $\sigma^{-}(L)$ pela Proposição 4.3.18, é um conjunto de números reais negativos. Se segue de (4.3.14) que

$$
0>\max _{\lambda \in \sigma\left(\left.L\right|_{\left.\operatorname{Im} P_{-}\right)}\right.} \lambda=\sup _{\|x\|=1}\left\langle\left. L\right|_{\operatorname{Im} P_{-}} x, x\right\rangle \quad \text { para } x \in \operatorname{Im} P_{-},
$$


isto é, $\left.L\right|_{\operatorname{Im} P_{-}}$é definida negativa. Consequentemente,

$$
\langle L x, x\rangle<0 \quad \text { para todo } x \in \operatorname{Im} P_{-}, x \neq 0 .
$$

Analogamente, de (4.3.14),

$$
0 \leq \min _{\lambda \in \sigma\left(\left.L\right|_{\text {Ker } P_{-}}\right)} \lambda=\inf _{\|x\|=1}\left\langle\left. L\right|_{\text {Ker } P_{-}} x, x\right\rangle \quad \text { para todo } x \in \operatorname{Ker} P_{-},
$$

isto é,

$$
\langle L x, x\rangle \geq 0 \quad \text { para todo } x \in \operatorname{Ker} P_{-} .
$$

Dado que a soma $H=\operatorname{Im} P_{-} \oplus \operatorname{Ker} P_{-}$é ortogonal, se segue de (4.3.17), (4.3.18) e do Teorema 3.3.19 que $\operatorname{Im} P_{-}=H_{-}(L)$, Ker $P_{-}=H_{+}(L) \oplus \operatorname{Ker} L$ e que $P$ é a projeção ortogonal sobre $H_{-}(L)$.

De forma análoga, a projeção ortogonal sobre $H_{+}(L)$ pode ser expressada da forma dada em (4.3.11).

A projeção apresentada em (4.3.11) somente é válida para o caso em que o espaço seja complexo. Usando a complexifição de um operador em um espaço de Hilbert real, podemos representar tal projeção de forma similar. Suponhamos que $H$ seja um espaço de Hilbert real e $L \in L(H)$ seja um operador auto-adjunto. Seja $\widehat{L} \in L(\widehat{H})$ a complexificação de L. Assim,

$$
\widehat{H}=H_{+}(\widehat{L}) \oplus H_{-}(\widehat{L}) \oplus \operatorname{Ker}(\widehat{L}) .
$$

Se $\widehat{x}=x+i y \in \widehat{H}$, onde $x, y \in H$, a parte real de $\widehat{x}$ é definida por $\operatorname{Re} \widehat{x}=x$.

Para um subconjunto $\Omega$ de $\widehat{H}$, definamos

$$
\operatorname{Re}(\Omega)=\{\operatorname{Re} \widehat{x}: \widehat{x} \in \Omega\} .
$$

É claro que $\widehat{L}$ e $L$ coincidem em $\operatorname{Re}(\Omega)$ para qualquer subconjunto $\Omega$ de $\widehat{H}$. Identifica$\operatorname{remos} L: H \rightarrow H$ com a restrição $\left.\widehat{L}\right|_{\operatorname{Re}(\widehat{H})}: \operatorname{Re}(\widehat{H}) \rightarrow \operatorname{Re}(\widehat{H})$.

Vejamos que $\widehat{L}\left(\overline{H_{+}(\widehat{L})}\right) \subseteq \overline{H_{+}(\widehat{L})}$, onde

$$
\overline{H_{+}(\widehat{L})}=\left\{x_{1}-i x_{2}: x_{1}+i x_{2} \in H_{+}(\widehat{L}), x_{1}, x_{2} \in H\right\} .
$$

De fato, seja $\widehat{x}=x_{1}+i x_{2} \in H_{+}(\widehat{L})$, com $x_{1} \in H$ e $x_{2} \in H$. Daí,

$$
\widehat{L} \widehat{\widehat{x}}=\widehat{L}\left(x_{1}-i x_{2}\right)=L x_{1}-i L x_{2}=\overline{L x_{1}+i L x_{2}}=\overline{\widehat{L} \widehat{x}} .
$$

Como $\widehat{L} \widehat{x} \in H_{+}(\widehat{L})$, temos $\overline{\widehat{L} \widehat{x}} \in \overline{H_{+}(\widehat{L})}$, como queríamos provar. 
Mostremos que $\widehat{L}$ é definido positivo em $\overline{H_{+}(\widehat{L})}$. Seja $\overline{\widehat{x}} \in \overline{H_{+}(\widehat{L})}$, onde $\widehat{x} \in H_{+}(\widehat{L})$ com $\widehat{x} \neq 0$. De (3.3.3) obtemos que $\langle\overline{\widehat{L} \widehat{x}}, \overline{\widehat{x}}\rangle=\overline{\langle\widehat{L} \widehat{x}, \widehat{x}\rangle}$. Logo, como $\langle\widehat{L} \widehat{x}, \widehat{x}\rangle>0$,

$$
\langle\widehat{L} \bar{x}, \overline{\widehat{x}}\rangle=\langle\overline{\mathrm{L}} \widehat{x}, \overline{\widehat{x}}\rangle=\overline{\langle\widehat{L} \widehat{x}, \widehat{x}\rangle}>0 .
$$

Analogamente, $\widehat{L}$ é definido negativo em $\overline{H_{-}(\widehat{L})}$. É claro que $\overline{\operatorname{Ker}(\widehat{L})}=\operatorname{Ker}(\widehat{L})$. Assim, se segue do Teorema 3.3.19 que

$$
\overline{H_{+}(\widehat{L})}=H_{+}(\widehat{L}) \quad \text { e } \overline{H_{-}(\widehat{L})}=H_{-}(\widehat{L}) .
$$

Lema 4.3.20. Com as condições acima,

$$
H=\operatorname{Re}\left(H_{+}(\widehat{L})\right) \oplus \operatorname{Re}\left(H_{-}(\widehat{L})\right) \oplus \operatorname{Re}(\operatorname{Ker}(\widehat{L})),
$$

e, além disso,

$$
H_{+}(L)=\operatorname{Re}\left(H_{+}(\widehat{L})\right), \quad H_{-}(L)=\operatorname{Re}\left(H_{-}(\widehat{L})\right) \quad e \operatorname{Ker}(L)=\operatorname{Re} \operatorname{Ker}(\widehat{L}) .
$$

Demonstração. Seja $x \in H$ dado. Logo, $\widehat{x}=x+i 0 \in \widehat{H}$. Portanto, existem $\widehat{x}_{+} \in$ $H_{+}(\widehat{L}), \widehat{x}_{-} \in H_{+}(\widehat{L})$ e $\widehat{x}_{0} \in \operatorname{Ker}(\widehat{L})$ únicos tais que

$$
\widehat{x}=\widehat{x}_{+}+\widehat{x}_{-}+\widehat{x}_{0} .
$$

Daí,

$$
\widehat{x}=\operatorname{Re} \widehat{x}=\operatorname{Re} \widehat{x}_{+}+\operatorname{Re} \widehat{x}_{-}+\operatorname{Re} \widehat{x}_{0} .
$$

De (4.3.19) se segue $\operatorname{Re} \widehat{x}_{+}=\left(\widehat{x}_{+}+\overline{\widehat{x}_{+}}\right) / 2 \in H_{+}(\widehat{L}), \operatorname{Re} \widehat{x}_{-}=\left(\widehat{x}_{-}+\overline{\widehat{x}_{-}}\right) / 2 \in H_{-}(\widehat{L}) \mathrm{e}$ $\operatorname{Re} \widehat{x}_{0}=\left(\widehat{x}_{0}+{\widehat{x_{0}}}_{0}\right) / 2 \in \operatorname{Ker}(\widehat{L})$. De (4.3.22) e da unicidade da soma (4.3.21) temos que $\operatorname{Re} \widehat{x}_{+}=\widehat{x}_{+}, \operatorname{Re} \widehat{x}_{-}=\widehat{x}_{-}$e $\operatorname{Re} \widehat{x}_{0}=\widehat{x}_{0}$. Este fato prova que

$$
H=\operatorname{Re}\left(H_{+}(\widehat{L})\right)+\operatorname{Re}\left(H_{-}(\widehat{L})\right)+\operatorname{Re}(\operatorname{Ker}(\widehat{L})) .
$$

Além disso, para $x \in \operatorname{Re}\left(H_{+}(\widehat{L})\right)$,

$$
L x=\widehat{L} \operatorname{Rex}=\operatorname{Re} \widehat{L} x \in \operatorname{Re}\left(H_{+}(\widehat{L})\right) .
$$

Logo, $\operatorname{Re}\left(H_{+}(\widehat{L})\right)$ é invariante por $L$. De igual forma podemos provar que $\operatorname{Re}\left(H_{-}(\widehat{L})\right)$ é invariante por $L$.

Observe que

$$
\operatorname{Re}\left(H_{+}(\widehat{L})\right)=\left\{(\widehat{x}+\widehat{\widehat{x}}) / 2: \widehat{x} \in H_{+}(\widehat{L})\right\} \subseteq H_{+}(\widehat{L})+\overline{H_{+}(\widehat{L})}=H_{+}(\widehat{L}) .
$$

Consequentemente, $L$ é definido positivo em $\operatorname{Re}\left(H_{+}(\widehat{L})\right)$. Analogamente,

$$
\operatorname{Re}\left(H_{-}(\widehat{L})\right) \subseteq H_{-}(\widehat{L}) \quad \text { e } \quad \operatorname{Re} \operatorname{Ker}(\widehat{L}) \subseteq \operatorname{Ker}(\widehat{L}) .
$$

Os fatos acima mostram a decomposição (4.3.20) e, além disso,

$$
H_{+}(L)=\operatorname{Re}\left(H_{+}(\widehat{L})\right), \quad H_{-}(L)=\operatorname{Re}\left(H_{-}(\widehat{L})\right) \quad \text { e } \operatorname{Ker}(L)=\operatorname{Re} \operatorname{Ker}(\widehat{L}),
$$

o que prova o lema. 
Sejam $L \in L(H)$ um operador auto-adjunto em um espaço de Hilbert real $H$ tal que 0 não seja ponto de acumulação de $\sigma(L)$. Tomemos uma curva fechada $\Gamma$ que contém no seu interior o conjunto $\sigma^{-}(L)$ e no seu exterior o conjunto $\{0\} \cap \sigma^{+}(L)$. Como vimos acima,

$$
P=-\frac{1}{2 \pi i} \int_{\Gamma}(\widehat{L}-\lambda I)^{-1} d \lambda
$$

é a projeção ortogonal sobre $H_{-}(\widehat{L})$. Daí, a restrição

$$
\left.P\right|_{\operatorname{Re} \widehat{H}}: \operatorname{Re} \widehat{H} \rightarrow \operatorname{Re} \widehat{H}
$$

do operador $P$ é a projeção ortogonal sobre $\operatorname{Re} H_{-}(\widehat{L})=H_{-}(L)$. Abusando um pouco da notação, no caso em que $H$ seja um espaço de Hilbert real, identificaremos tal restrição com a projeção dada em (4.3.23).

Concluiremos esta seção com dois resultados que têm a ver com a continuidade das projeções ortogonais. Antes disso, vejamos primeiro o seguinte lema, cuja prova se pode encontrar, por exemplo, em [15], pág. 45, Teorema 5.

Lema 4.3.21. Se $f: \Delta \rightarrow E$ é regular, onde $E$ é um espaço de Banach, então, para qualquer caminho retificável $\Gamma \subseteq \Delta$,

$$
\left\|\int_{\Gamma} f(\lambda) d \lambda\right\| \leq M l
$$

onde $M=\sup _{\lambda \in \Gamma}|f(\lambda)|$ e l é o comprimento de $\Gamma$.

Teorema 4.3.22. Sejam $J=[a, b] \subseteq \mathbb{R} e L: J \rightarrow L_{S}(H)$ um caminho de operadores auto-adjuntos. Suponhamos que exista $c \in \mathbb{R}$ tal que, para cada $\lambda \in J$,

$$
\sigma\left(L_{\lambda}\right) \subseteq(-\infty, c) \cup(c,+\infty) .
$$

Então, para $\lambda \in J$, a projeção $P_{\lambda}$ sobre o subespaço espectral de $L_{\lambda}$ correspondente a $\sigma\left(L_{\lambda}\right) \cap(-\infty, c)$ existe e, além disso,

$$
\begin{aligned}
P: J & \rightarrow L_{S}(H) \\
\lambda & \mapsto P_{\lambda}
\end{aligned}
$$

é um caminho de projeções ortogonais.

Demonstração. É claro que a aplicação $\varphi: J \rightarrow \mathbb{R}$ definida por

$$
\lambda \mapsto \varphi(\lambda)=\left\|\mathrm{L}_{\lambda}\right\|=\sup _{\|x\|=1}\left|\left\langle\mathrm{~L}_{\lambda} x, x\right\rangle\right|
$$


é contínua. Já que $J$ é compacto, existe

$$
l=\max _{\lambda \in J} \varphi(\lambda) \in \mathbb{R} .
$$

Se segue da Proposição 3.3.6 que

$$
\sigma\left(\mathrm{L}_{\lambda}\right) \subseteq[-l, l] \text { para } \lambda \in J .
$$

Agora, se $c \leq-l$, então $\mathbf{P}_{\lambda}=0$ para todo $\lambda \in J$, pois $\sigma\left(\mathrm{L}_{\lambda}\right) \cap(-\infty, c)=\emptyset$. Neste caso o teorema é claramente válido.

Por outro lado, suponhamos que $c>l$. Sejam

$$
\sigma_{1}=\bigcup_{\lambda \in J}\left[\sigma\left(\mathrm{L}_{\lambda}\right) \cap(-\infty, c)\right] \quad \text { e } \quad \sigma_{2}=\bigcup_{\lambda \in J}\left[\sigma\left(\mathrm{L}_{\lambda}\right) \cap(c,+\infty)\right] .
$$

Mostremos que $c$ não é ponto de acumulação do conjunto

$$
\sigma=\sigma_{1} \cup \sigma_{2}=\bigcup_{\lambda \in J}\left[\sigma\left(\mathrm{L}_{\lambda}\right) \cap(-\infty, c)\right] \cup \bigcup_{\lambda \in J}\left[\sigma\left(\mathrm{L}_{\lambda}\right) \cap(c,+\infty)\right]=\bigcup_{\lambda \in J} \sigma\left(\mathrm{L}_{\lambda}\right) .
$$

De fato, suponhamos por absurdo que $c$ seja ponto de acumulação de $\sigma$. Assim, existe uma sequência $\left(\alpha_{n}\right)_{n=1}^{\infty}$ contida em $\sigma$ que converge a $c$. Pela definição de $\sigma$, existe uma sequência de operadores $\left(\mathrm{L}_{\lambda_{n}}\right)_{n=1}^{\infty} \subseteq \operatorname{Im} \mathrm{L}$, tal que $\alpha_{n} \in \sigma\left(\mathrm{L}_{\lambda_{n}}\right)$ para todo $n$. Como $J$ é compacto, $\left(\mathrm{L}_{\lambda_{n}}\right)_{n=1}^{\infty}$ possui uma subsequência convergente a $\mathrm{L}_{\lambda} \in \operatorname{Im} \mathrm{L}$. Sem perda de generalidade, podemos dar o mesmo nome a esta subsequência. Vejamos que $c \in \sigma\left(\mathrm{L}_{\lambda}\right)$. De fato, suponhamos, novamente por absurdo, que $c \notin \sigma\left(\mathrm{L}_{\lambda}\right)$. Então, $\mathrm{L}_{\lambda}-c I$ é inversível. Dado que o conjunto dos operadores inversíveis é aberto e $\left(\mathrm{L}_{\lambda_{n}}-\alpha_{n} I\right)_{n=1}^{\infty}$ converge a $\mathrm{L}_{\lambda}-c I$, existe $N \in \mathbb{N}$ tal que $\mathrm{L}_{\lambda_{n}}-\alpha_{n} I$ é inversível para todo $n>N$. Este fato contradiz que $\alpha_{n} \in \sigma\left(\mathrm{L}_{\lambda_{n}}\right)$ para todo $n$. Logo, $c \in \sigma\left(\mathrm{L}_{\lambda}\right)$, contradizendo nossa hipótese que, para $\lambda \in J, c \notin \sigma\left(\mathrm{L}_{\lambda}\right)$. Em conclusão, $c$ não é ponto de acumulação de $\sigma$.

Por outro lado, de (4.3.24) e do fato anterior se segue que existem $a_{1}, a_{2} \in \mathbb{R}$, com $-l<a_{1}<c<a_{2}<l$, tais que

$$
\sigma_{1} \subseteq B\left(-l, a_{1}+l\right) \quad \text { e } \quad \sigma_{2} \subseteq B\left(l, l-a_{2}\right) .
$$

Consequentemente,

$$
\Gamma_{1}(t)=(-l, 0)+\left(a_{1}+l\right) e^{2 \pi i t} \quad \text { e } \quad \Gamma_{2}(t)=(l, 0)+\left(l-a_{2}\right) e^{2 \pi i t} \text {, para } t \in[0,1],
$$

são curvas fechadas tais que $\sigma_{1} \subseteq \stackrel{\circ}{\Gamma}_{1}$ e $\sigma_{2} \subseteq \stackrel{\circ}{\Gamma}_{2}$. Tomemos

$$
\Delta_{1}=\{(x, y) \in \mathbb{C}: x<c\} \quad \text { e } \quad \Delta_{2}=\{(x, y) \in \mathbb{C}: x>c\} .
$$

Logo, $\sigma_{1} \subseteq \stackrel{\circ}{\Gamma}_{1} \subseteq \Delta_{1}$ e $\sigma_{2} \subseteq \stackrel{\circ}{\Gamma}_{2} \subseteq \Delta_{2}$. Definindo a aplicação $\chi: \Delta_{1} \cup \Delta_{2} \rightarrow \mathbb{C}$ como

$$
\chi(z)= \begin{cases}1 & \text { se } z \in \Delta_{1} \\ 0 & \text { se } z \in \Delta_{2},\end{cases}
$$


temos que, se $\lambda \in J$,

$$
\mathrm{P}_{\lambda}=-\frac{1}{2 \pi i} \int_{\Gamma_{1}}\left(\mathrm{~L}_{\lambda}-\zeta I\right)^{-1} d \zeta
$$

é a projeção ortogonal sobre o subespaço espectral de $L_{\lambda}$ correspondente a $\sigma\left(L_{\lambda}\right) \cap$ $(-\infty, c)$.

Agora provemos a continuidade de P. Fixemos $\lambda_{0}$ em $J$. De (1.1.1) obtemos

$$
\left(\mathrm{L}_{\lambda_{0}}-\zeta I\right)^{-1}-\left(\mathrm{L}_{\lambda}-\zeta I\right)^{-1}=-\left(\mathrm{L}_{\lambda_{0}}-\zeta I\right)^{-1}\left(\mathrm{~L}_{\lambda_{0}}-\mathrm{L}_{\lambda}\right)\left(\mathrm{L}_{\lambda}-\zeta I\right)^{-1} \quad \text { para } \zeta \in \Gamma_{1} .
$$

Assim,

$$
\begin{aligned}
\mathrm{P}_{\lambda_{0}}-\mathrm{P}_{\lambda} & =-\frac{1}{2 \pi i} \int_{\Gamma_{1}}\left(\mathrm{~L}_{\lambda_{0}}-\zeta I\right)^{-1} d \zeta+\frac{1}{2 \pi i} \int_{\Gamma_{1}}\left(\mathrm{~L}_{\lambda}-\zeta I\right)^{-1} d \zeta \\
& =-\frac{1}{2 \pi i} \int_{\Gamma_{1}}\left(\left(\mathrm{~L}_{\lambda_{0}}-\zeta I\right)^{-1}-\left(\mathrm{L}_{\lambda}-\zeta I\right)^{-1}\right) d \zeta \\
& =\frac{1}{2 \pi i} \int_{\Gamma_{1}}\left(\mathrm{~L}_{\lambda_{0}}-\zeta I\right)^{-1}\left(\mathrm{~L}_{\lambda_{0}}-\mathrm{L}_{\lambda}\right)\left(\mathrm{L}_{\lambda}-\zeta I\right)^{-1} d \zeta .
\end{aligned}
$$

É fácil ver que $\phi: J \times \Gamma_{1} \rightarrow L(H)$, definida por $\phi(\lambda, \zeta)=\left(\mathrm{L}_{\lambda}-\zeta I\right)$, é contínua. Portanto, do Lema 1.1.9 se segue que a aplicação $(\lambda, \zeta) \mapsto\left(\mathrm{L}_{\lambda}-\zeta I\right)^{-1}$ é contínua em $J \times \Gamma_{1}$, sendo composição de aplicações contínuas. Daí, como $J \times \Gamma_{1}$ é compacto, existe $M>0$ tal que

$$
\sup _{(\lambda, \zeta) \in J \times \Gamma_{1}}\left\|\left(\mathrm{~L}_{\lambda}-\zeta I\right)^{-1}\right\| \leq M .
$$

Assim,

$$
\left\|\left(\mathrm{L}_{\lambda_{0}}-\zeta I\right)^{-1}\left(\mathrm{~L}_{\lambda_{0}}-\mathrm{L}_{\lambda}\right)\left(\mathrm{L}_{\lambda}-\zeta I\right)^{-1}\right\| \leq M^{2}\left\|\mathrm{~L}_{\lambda_{0}}-\mathrm{L}_{\lambda}\right\| .
$$

Logo, se $\left\|\mathrm{L}_{\lambda_{0}}-\mathrm{L}_{\lambda}\right\|<\varepsilon$, para qualquer $\varepsilon>0$, então, pelo Lema 4.3.21,

$$
\left\|\mathrm{P}_{\lambda_{0}}-\mathrm{P}_{\lambda}\right\| \leq \frac{1}{2 \pi} M^{2} l \varepsilon
$$

onde $l$ é o comprimento de $\Gamma$. Consequentemente, $\mathrm{P}$ é contínua.

Da prova do teorema anterior temos o seguinte corolário.

Corolário 4.3.23. A aplicação $P: G L_{S}(H) \rightarrow L(H)$ que associa a cada operador $L \in$ $G l_{S}(H)$ a projeção ortogonal $P_{H_{-}(L)}$ sobre seu subespaço espectral negativo é contínua.

Demonstração. Seja $L \in G L_{S}(H)$ fixado. Assim, existe $c>0$ tal que $\|L x\| \geq c\|x\|$ para todo $x \in H$. Seja $B(L, c / 2) \subseteq G L_{S}(H)$ a bola aberta com centro em $L$ e raio $c / 2$ e tomemos $T \in B(L, c / 2)$. Mostremos que, se $\lambda \in \mathbb{R}$ com $|\lambda|<c / 2$, então $\lambda \in \rho(T)$. De fato, fixemos $\lambda \in \mathbb{R} \operatorname{com}|\lambda|<c / 2$ e $z \in H$ de norma 1 . Observe que

$$
\|T z\| \geq\|L z\|-\|L z-T z\| \geq c-c / 2,
$$


isto é, $\|T z\| \geq c / 2$. Consequentemente,

$$
\|(T-\lambda I) z\| \geq\|T z\|-\|\lambda z\| \geq \frac{c}{2}-|\lambda|>0 .
$$

Este fato mostra que $\operatorname{Ker}(T-\lambda I)=\{0\}$. Logo, a Proposição 1.1.10 implica que a imagem de $T-\lambda I$ é fechada. Além disso, como $T-\lambda I$ é auto-adjunto, das Proposições 3.1.5 e 3.2.9 obtemos que $\operatorname{Im}(T-\lambda I)=H$. Em conclusão, $\lambda \in \rho(T)$.

Por outro lado, já que $\|T\| \leq\|L\|+c / 2$, então, para $\lambda \in \mathbb{R}$ com $|\lambda|>\|L\|-c / 2$, $\lambda \in \rho(T)$ pela Proposição 3.3.6. Os fatos acima mostram que

$$
\sigma(T) \subseteq[-(\|L\|+c / 2),-c / 2] \cup[c / 2,\|L\|+c / 2] \quad \text { para todo } T \in B(L, c / 2) .
$$

Como provamos no Teorema anterior, existem duas curvas fechadas disjuntas $\Gamma_{1}$ e $\Gamma_{2}$ tais que $\sigma^{-}(T) \subseteq \stackrel{\circ}{\Gamma}_{1}, \sigma^{+}(T) \subseteq \stackrel{\circ}{\Gamma}_{2} \mathrm{e}$

$$
\mathrm{P}_{H_{-}(T)}=-\frac{1}{2 \pi i} \int_{\Gamma_{1}}(T-\zeta I)^{-1} d \zeta
$$

é a projeção ortogonal sobre o subespaço espectral de $T$ correspondente a $\sigma^{-}(T)$, isto é, $\mathrm{P}_{H_{-}(T)}$ é a projeção ortogonal sobre $H_{-}(T)$. A continuidade de $\mathrm{P}$ se segue da prova do teorema anterior.

\subsection{Pares de operadores de Fredholm e índice de Morse relativo}

Lembremos que o índice de Morse de um operador essencialmente positivo $L \in L(H)$ é definido como a dimensão do seu subespaço espectral negativo, e que o denotamos por $\mu(L)$. Como já foi dito, o fluxo espectral é um conceito que é introduzido quando o índice de Morse não existe. Aqui apresentaremos um conceito que é chamado de indice de Morse relativo, que é definido para pares de isomorfismos auto-adjuntos cuja diferença é compacta. No próximo capítulo veremos que o índice de Morse relativo e o fluxo espectral estão estritamente ligados.

Para definir o índice de Morse relativo, introduziremos primeiro a noção de "par de Fredholm"e seu índice, e usaremos as propriedades dos operadores congruentes módulo operador compacto, que foram apresentadas no Capítulo 2.

Sejam $H$ um espaço de Hilbert e $(L, T)$ um par de isomorfismos auto-adjuntos em $L(H)$. Veremos que, se $H$ tem dimensão finita, o indice de Morse relativo do par $(L, T)$ coincide com a diferença dos índices de Morse de $L$ e $T$, isto é, $\mu(L)-\mu(T)$.

No final da seção veremos uma relação que liga o índice de Morse relativo com a assinatura generalizada de uma perturbação compacta auto-adjunta de uma simetria, definida na Seção 2 deste capítulo. 
Definição 4.4.1. Dizemos que um par de projeções ortogonais $(P, Q)$ é um par de Fredholm se $\operatorname{Im} P \cap \operatorname{Ker} Q$ e $\operatorname{Im} Q \cap \operatorname{Ker} P$ têm dimensão finita. $\mathrm{O}$ indice de $(P, Q)$ é definido por

$$
\operatorname{ind}(P, Q)=\operatorname{dim}(\operatorname{Im} P \cap \operatorname{Ker} Q)-\operatorname{dim}(\operatorname{Im} Q \cap \operatorname{Ker} P) .
$$

Apresentaremos na seguinte proposição uma relação que liga os operadores congruentes módulo operador compacto aos pares de Fredholm.

Proposição 4.4.2. Seja $(P, Q)$ um par de projeções ortogonais em $L(H)$. Se $P-Q$ é compacto, então $(P, Q)$ é um par de Fredholm.

Demonstração. Denotemos por $K$ o operador compacto $P-Q$. Então,

$$
K=P-Q=P(I-Q)+P Q-Q=((I-Q) P)^{*}-(I-P) Q
$$

e

$$
(I-P) Q=(Q+I-Q-P) Q=(Q-P) Q=-K Q .
$$

Os dois fatos acima implicam que

$$
((I-Q) P))^{*}=(I-P) Q+K=-K Q+K=K(I-Q) .
$$

Logo,

$$
(I-Q) P=(K(I-Q))^{*}=(I-Q)^{*} K^{*}=(I-Q) K .
$$

Consequentemente, os operadores $(I-P) Q=-K Q$ e $(I-Q) P=(I-Q) K$ são compactos.

Agora, é fácil ver que $(I-P) Q$ restrito a $\operatorname{Im} Q \cap \operatorname{Ker} P$ é a identidade. Dado que a identidade de um espaço de Banach é compacta se, e somente se, seu domínio tem dimensão finita, temos que

$$
\operatorname{dim}(\operatorname{Im} Q \cap \operatorname{Ker} P)<\infty .
$$

Analogamente, $(I-Q) P$ restrito a $\operatorname{Im} P \cap \operatorname{Ker} Q$ é a identidade e portanto

$$
\operatorname{dim}(\operatorname{Im} P \cap \operatorname{Ker} Q)<\infty .
$$

Assim, $(P, Q)$ é um par de Fredholm.

Em seguida veremos algumas das propriedades que possui o índice de um par de Fredholm.

Lema 4.4.3. Se $(P, Q)$ é um par de Fredholm em $L(H)$ e o operador $O \in L(H)$ é ortogonal, então $\left(O^{*} P O, O^{*} Q O\right)$ é um par de Fredholm e

$$
\operatorname{ind}(P, Q)=\operatorname{ind}\left(O^{*} P O, O^{*} Q O\right) .
$$


Demonstração. Sejam $\widetilde{P}=O^{*} P O$ e $\widetilde{Q}=O^{*} Q O$. Então,

$$
\widetilde{P}^{*}=\left(O^{*} P O\right)^{*}=O^{*} P^{*} O=O^{*} P O=\widetilde{P},
$$

isto é, $\widetilde{P}$ é auto-adjunto. Analogamente, $\widetilde{Q}$ é auto-adjunto. Dado que $\widetilde{P}^{2}=\widetilde{P}$ e $\widetilde{Q}^{2}=\widetilde{Q}$, se segue da Proposição 3.2.13 que $\widetilde{P}$ e $\widetilde{Q}$ são projeções ortogonais.

É fácil ver que $\operatorname{Ker} \widetilde{P}=O^{*}(\operatorname{Ker} P)$ e $\operatorname{Ker} \widetilde{Q}=O^{*}(\operatorname{Ker} Q)$. Assim, como $\operatorname{Im} O=H$ e $O^{*}=O^{-1}$, temos que

$$
\begin{aligned}
\operatorname{Im} \widetilde{P} \cap \operatorname{Ker} \widetilde{Q} & =\operatorname{Im}\left(O^{*} P O\right) \cap O^{*}(\operatorname{Ker} Q)=O^{*}(\operatorname{Im} P O) \cap O^{*}(\operatorname{Ker} Q) \\
& =O^{*}[(\operatorname{Im} P O) \cap(\operatorname{Ker} Q)]=O^{*}[P(\operatorname{Im} O) \cap(\operatorname{Ker} Q)] \\
& =O^{*}[\operatorname{Im} P \cap \operatorname{Ker} Q] .
\end{aligned}
$$

Logo,

$$
\operatorname{dim}(\operatorname{Im} \widetilde{P} \cap \operatorname{Ker} \widetilde{Q})=\operatorname{dim}(\operatorname{Im} P \cap \operatorname{Ker} Q) .
$$

Analogamente,

$$
\operatorname{dim}(\operatorname{Im} \widetilde{Q} \cap \operatorname{Ker} \widetilde{P})=\operatorname{dim}(\operatorname{Im} Q \cap \operatorname{Ker} P) .
$$

Os dois fatos acima mostram que $\left(O^{*} P O, O^{*} Q O\right)$ é um par de Fredholm e que, além disso, $\operatorname{ind}(P, Q)=\operatorname{ind}\left(O^{*} P O, O^{*} Q O\right)$.

$\mathrm{Na}$ Proposição 4.4.5 provaremos que o índice de um caminho de um par de projeções de Fredholm é constante. Para este fim, vejamos primeiro o seguinte lema.

Lema 4.4.4. Se $(P, Q)$ é um par de projeções de Fredholm, então o complementar ortogonal de $Q(\operatorname{Im} P)$ em $\operatorname{Im} Q$ é $\operatorname{Im} Q \cap \operatorname{Ker} P$.

Demonstração. Seja $z \in \operatorname{Im} Q \cap \operatorname{Ker} P$ fixado. Assim, $Q z=z$ e $P z=0$. Se $y \in$ $Q(\operatorname{Im} P)$, então $y=Q P x$ para algum $x \in H$. Agora,

$$
\langle y, z\rangle=\langle Q P x, z\rangle=\langle P x, Q z\rangle=\langle P x, z\rangle=\langle x, P z\rangle=0 .
$$

Logo, $z \in(Q(\operatorname{Im} P))^{\perp}$, isto é,

$$
\operatorname{Im} Q \cap \operatorname{Ker} P \subseteq(Q(\operatorname{Im} P))^{\perp} .
$$

Por outro lado, seja $z \in \operatorname{Im} Q$ tal que $\langle x, z\rangle=0$ para todo $x \in Q(\operatorname{Im} P)$. Então, $\langle Q P y, z\rangle=0$ para todo $y \in H$. Daí,

$$
0=\langle Q P y, z\rangle=\langle P y, Q z\rangle=\langle P y, z\rangle=\langle y, P z\rangle \quad \text { para todo } y \in H .
$$

Logo, $P z=0$, isto é, $z \in \operatorname{Ker} P$. Consequentemente,

$$
\operatorname{Im} Q \cap(Q(\operatorname{Im} P))^{\perp} \subseteq \operatorname{Im} Q \cap \operatorname{Ker} P .
$$

De (4.4.1) e (4.4.2) temos $\operatorname{Im} Q \cap(Q(\operatorname{Im} P))^{\perp}=\operatorname{Im} Q \cap \operatorname{Ker} P$, o que prova o lema. 
Do lema anterior se segue que

$$
\operatorname{Im} Q=Q(\operatorname{Im} P) \oplus(\operatorname{Im} Q \cap \operatorname{Ker} P) .
$$

Seja agora $\hat{Q}: \operatorname{Im} P \rightarrow \operatorname{Im} Q$ a restrição de $Q$ a $\operatorname{Im} P$. É claro que

$$
\operatorname{dim} \operatorname{Ker} \hat{Q}=\operatorname{dim}(\operatorname{Ker} Q \cap \operatorname{Im} P),
$$

pois $\operatorname{Ker} \hat{Q}=\operatorname{Ker} Q \cap \operatorname{Im} P$. Além disso, do Lema 4.4.4 temos $(\operatorname{Im} \hat{Q})^{\perp}=\operatorname{Im} Q \cap \operatorname{Ker} P$. Assim,

$$
\operatorname{dim} \operatorname{coKer} \hat{Q}=\operatorname{dim}(\operatorname{Im} Q \cap \operatorname{Ker} P) .
$$

De (4.4.3) e (4.4.4) se segue que $\hat{Q}: \operatorname{Im} P \rightarrow \operatorname{Im} Q$ é um operador de Fredholm de índice

$$
\text { ind } \hat{Q}=\operatorname{dim}(\operatorname{Ker} Q \cap \operatorname{Im} P)-\operatorname{dim}(\operatorname{Im} Q \cap \operatorname{Ker} P) \text {. }
$$

Este fato implica que $\operatorname{ind}(P, Q)$ coincide com o índice de $\hat{Q}: \operatorname{Im} P \rightarrow \operatorname{Im} Q$.

No resto da seção, $J$ denotará um intervalo $[a, b]$ e $\left\{\left(P_{t}, Q_{t}\right)\right\}_{t \in J}$ um caminho contínuo de pares de Fredholm, no sentido de que $t \mapsto P_{t}$ e $t \mapsto Q_{t}$ são contínuas.

Proposição 4.4.5. O indice do caminho $\left\{\left(P_{t}, Q_{t}\right)\right\}_{t \in J}$ é constante.

Demonstração. Para provar que ind $\left(P_{t}, Q_{t}\right)$ é constante é suficiente provar que ele é localmente constante, pois $J$ é conexo. Para este fim, tomemos $t_{0} \in J$ fixado. Da continuidade de $t \mapsto P_{t}$ e $t \mapsto Q_{t}$ temos que

$$
\left\{A_{t}=\left(I-P_{t}\right)\left(I-P_{t_{0}}\right)+P_{t} P_{t_{0}}\right\}_{t \in J} \quad \text { e } \quad\left\{B_{t}=\left(I-Q_{t}\right)\left(I-Q_{t_{0}}\right)+Q_{t} Q_{t_{0}}\right\}_{t \in J}
$$

são contínuos. Sendo $A_{t_{0}}=B_{t_{0}}=I$, existe uma vizinhança conexa $\Omega$ de $t_{0}$ em $J$ tal que $A_{t}$ e $B_{t}$ são isomorfismos para $t \in \Omega$ e, assim, as restrições $A_{t}: \operatorname{Im} P_{t_{0}} \rightarrow A_{t}\left(\operatorname{Im} P_{t_{0}}\right)$ e $B_{t}: \operatorname{Im} Q_{t_{0}} \rightarrow B_{t}\left(\operatorname{Im} Q_{t_{0}}\right)$ são isomorfismos.

Mostremos que

$$
A_{t}\left(\operatorname{Im} P_{t_{0}}\right)=\operatorname{Im} P_{t} \quad \text { e } \quad B_{t}\left(\operatorname{Im} Q_{t_{0}}\right)=\operatorname{Im} Q_{t} .
$$

De fato, se $y \in \operatorname{Im} P_{t_{0}}$, então $y=P_{t_{0}} x$ para algum $x \in H$. Consequentemente,

$$
\begin{aligned}
A_{t} y & =\left(\left(I-P_{t}\right)\left(I-P_{t_{0}}\right)+P_{t} P_{t_{0}}\right) P_{t_{0}} x=\left(I-P_{t}\right)\left(I-P_{t_{0}}\right) P_{t_{0}} x+P_{t} P_{t_{0}} P_{t_{0}} x=P_{t} P_{t_{0}} x \\
& =P_{t} y .
\end{aligned}
$$

Assim,

$$
A_{t}\left(\operatorname{Im} P_{t_{0}}\right) \subseteq \operatorname{Im} P_{t}
$$

Agora, suponhamos que $y$ pertença a $\left(\operatorname{Im} P_{t_{0}}\right)^{\perp}$. Então, $P_{t_{0}} y=0$ e $\left(I-P_{t_{0}}\right) y=y$. Logo,

$$
A_{t} y=\left(I-P_{t}\right) y
$$


Este fato prova que

$$
A_{t}\left(\left(\operatorname{Im} P_{t_{0}}\right)^{\perp}\right) \subseteq\left(\operatorname{Im} P_{t}\right)^{\perp} .
$$

Dado que, para $t \in \Omega, A_{t}$ é um isomorfismo,

$$
H=A_{t}(H)=A_{t}\left[\operatorname{Im} P_{t_{0}} \oplus\left(\operatorname{Im} P_{t_{0}}\right)^{\perp}\right]=A_{t}\left(\operatorname{Im} P_{t_{0}}\right) \oplus A_{t}\left(\left(\operatorname{Im} P_{t_{0}}\right)^{\perp}\right) .
$$

Consequentemente,

$$
\operatorname{Im} P_{t} \oplus\left(\operatorname{Im} P_{t}\right)^{\perp}=H=A_{t}\left(\operatorname{Im} P_{t_{0}}\right) \oplus A_{t}\left(\left(\operatorname{Im} P_{t_{0}}\right)^{\perp}\right) .
$$

De (4.4.5), (4.4.6) e (4.4.7) temos

$$
A_{t}\left(\operatorname{Im} P_{t_{0}}\right)=\operatorname{Im} P_{t} \quad \text { e } \quad A_{t}\left(\left(\operatorname{Im} P_{t_{0}}\right)^{\perp}\right)=\left(\operatorname{Im} P_{t}\right)^{\perp} .
$$

Analogamente,

$$
B_{t}\left(\operatorname{Im} Q_{t_{0}}\right)=\operatorname{Im} Q_{t} \quad \text { e } \quad B_{t}\left(\left(\operatorname{Im} Q_{t_{0}}\right)^{\perp}\right)=\left(\operatorname{Im} Q_{t}\right)^{\perp} .
$$

Da composição das restrições

$$
\hat{A}_{t}=\left.A_{t}\right|_{\operatorname{Im} P_{t_{0}}}: \operatorname{Im} P_{t_{0}} \rightarrow \operatorname{Im} P_{t}, \quad \hat{Q}_{t}=\left.Q_{t}\right|_{\operatorname{Im} P_{t}}: \operatorname{Im} P_{t} \rightarrow \operatorname{Im} Q_{t}
$$

e

$$
\left(\hat{B}_{t}\right)^{-1}=\left.B_{t}^{-1}\right|_{\operatorname{Im} Q_{t}}: \operatorname{Im} Q_{t} \rightarrow \operatorname{Im} Q_{t_{0}}
$$

obtemos o operador

$$
\left(\hat{B}_{t}\right)^{-1} \hat{Q}_{t} \hat{A}_{t}: \operatorname{Im} P_{t_{0}} \rightarrow \operatorname{Im} Q_{t_{0}} .
$$

Como consequência das fórmulas (4.4.3) e (4.4.4), se segue que $\hat{Q}_{t}$ é um operador de Fredholm com

$$
\text { ind } \hat{Q}_{t}=\operatorname{ind}\left(P_{t}, Q_{t}\right) \quad \text { para todo } t \in \Omega \text {. }
$$

Dado que $A_{t}$ e $B_{t}$ são isomorfismos, $\left(\hat{B}_{t}\right)^{-1} \hat{Q}_{t} \hat{A}_{t}$ é um operador de Fredholm que tem o mesmo índice de $\hat{B}_{t}$ para $t \in \Omega$. Já que

$$
\left\{\left(\hat{B}_{t}\right)^{-1} \hat{Q}_{t} \hat{A}_{t}\right\}_{t \in \Omega}
$$

é um caminho de operadores de Fredholm com o mesmo domínio e contradomínio, a continuidade do índice de Fredholm (Teorema 2.2.4) implica que ind $\left(\left(\hat{B}_{t}\right)^{-1} \hat{Q}_{t} \hat{A}_{t}\right)$ é constante em $\Omega$. Assim, o índice de Fredholm do caminho $\left\{\hat{Q}_{t}\right\}_{t \in \Omega}$ também é constante.

De (4.4.8) se segue que ind $\left(P_{t}, Q_{t}\right)$ é constante para todo $t \in \Omega$, como queríamos provar. 
Lembramos que na Seção 4 do Capítulo 2 deste trabalho foi introduzido o conceito de operadores congruentes módulo operador compacto. Por simplicidade, se $L$ e $T$ são operadores congruentes módulo operador compacto, diremos que $L$ e $T$ são congruentes. Sejam $L$ e $T$ em $L(H)$ dois isomorfismos auto-adjuntos congruentes. Da seguinte proposição se segue que faz sentido definir o índice do par $(L, T)$. Como veremos na próxima definição, este índice será chamado de índice de Morse relativo. Para um operador auto-adjunto $L \in L(H)$, denotaremos por $P_{H_{-}(L)}$ a projeção ortogonal sobre $H_{-}(L)$, o subespaço espectral negativo de $L$.

Proposição 4.4.6. Sejam $L$ e $T$ dois isomorfismos auto-adjuntos congruentes. Então, as projeções ortogonais sobre os espaços espectrais negativos de $L$ e $T$ são congruentes.

Demonstração. É imediata se os espectros negativos de $L$ e de $T$ são vazios, pois, neste caso, $P_{H_{-}(L)}=P_{H_{-}(T)}=0$. Suponhamos que $L$ tenha espectro negativo não vazio. Dado que $L$ e $T$ são isomorfismos, 0 não pertence nem é ponto de acumulação de $\sigma(L) \cup \sigma(T)$. Portanto, existe uma curva fechada $\Gamma$ (veja-se Definição 4.3.2) que contém no seu interior os espectros negativos de $L$ e $T$ e no seu exterior os seus espectros positivos. Daí,

$$
P_{H_{-}(L)}=-\frac{1}{2 \pi i} \int_{\Gamma}(L-\lambda I)^{-1} d \lambda \quad \text { e } \quad P_{H_{-}(T)}=-\frac{1}{2 \pi i} \int_{\Gamma}(T-\lambda I)^{-1} d \lambda .
$$

Assim,

$$
P_{H_{-}(L)}-P_{H_{-}(T)}=\frac{1}{2 \pi i} \int_{\Gamma}(L-\lambda I)^{-1}(T-L)(L-\lambda I)^{-1} d \lambda .
$$

Dado que $M_{\lambda}=(L-\lambda I)^{-1}(T-L)(L-\lambda I)^{-1}$ é compacto para cada $\lambda \in \Gamma$, as combinações lineares finitas dos $M_{\lambda}$ são operadores compactos. Como a integral é o limite de uma sequência formada de combinações lineares finitas dos $M_{\lambda}$ e o espaço dos operadores compactos é fechado, temos que $P_{H_{-}(L)}-P_{H_{-}(T)}$ é compacto, o que prova a proposição.

Na Proposição 4.4.2 provamos que, se a diferença de duas projeções ortogonais $P$ e $Q$ é compacta, então $(P, Q)$ é um par de Fredholm. Assim, da proposição anterior obtemos que, se $L$ e $T$ são isomorfismos auto-adjuntos tais que $L-T$ é compacto, $\left(P_{H_{-}(L)}, P_{H_{-}(T)}\right)$ é um par de Fredholm. Consequentemente,

$$
\operatorname{dim}\left(\operatorname{Im} P_{H_{-}(L)} \cap \operatorname{Ker} P_{H_{-}(T)}\right)<\infty \quad \text { e } \quad \operatorname{dim}\left(\operatorname{Im} P_{H_{-}(T)} \cap \operatorname{Ker} P_{H_{-}(L)}\right)<\infty .
$$

Portanto, a seguinte definição faz sentido.

Definição 4.4.7 (Índice de Morse relativo). Seja $(L, T)$ um par de isomorfismos autoadjuntos congruentes. O indice de Morse relativo do par $(L, T)$ é definido por

$$
\mu_{r e l}(L, T)=\operatorname{ind}\left(P_{H_{-}(L)}, P_{H_{-}(T)}\right) .
$$


Suponhamos que $H$ tenha dimensão finita. É claro que, se $(L, T)$ é um par de operadores auto-adjuntos, então $L$ e $T$ são congruentes. Veremos que se $L$ e $T$ são isomorfismos auto-adjuntos, o índice de Morse relativo do par $(L, T)$ coincide com a diferença dos índices de Morse de $L$ e de $T$, isto é,

$$
\mu_{r e l}(L, T)=\mu(L)-\mu(T) .
$$

Mais geralmente, se $H$ tem dimensão infinita e $L, T \in \Phi_{S}^{+}(H)$ são isomorfismos congruentes, mostraremos que a fórmula acima também é válida. Para provar estes fatos, vejamos primeiro o seguinte lema.

Lema 4.4.8. Sejam $U$ e $V$ dois subespaços de dimensão finita de H. Então,

$$
\operatorname{dim} U-\operatorname{dim} V=\operatorname{dim}\left(U \cap V^{\perp}\right)-\operatorname{dim}\left(V \cap U^{\perp}\right) .
$$

Demonstração. Primeiro vejamos que $V^{\perp} \cap U=\left(V+U^{\perp}\right)^{\perp}$. De fato, tomemos $x \in$ $V^{\perp} \cap U$ fixado. Para $w \in V+U^{\perp}, w=w_{V}+w_{U^{\perp}}$, onde $w_{V} \in V$ e $w_{U^{\perp}} \in U^{\perp}$. Logo,

$$
\langle x, w\rangle=\left\langle x, w_{V}+w_{U^{\perp}}\right\rangle=\left\langle x, w_{V}\right\rangle+\left\langle x, w_{U^{\perp}}\right\rangle=0 .
$$

Este fato prova que $x \in\left(V+U^{\perp}\right)^{\perp}$. Daí,

$$
V^{\perp} \cap U \subseteq\left(V+U^{\perp}\right)^{\perp} .
$$

Agora, dado que $U^{\perp} \subseteq V+U^{\perp}$, então

$$
\left(V+U^{\perp}\right)^{\perp} \subseteq U^{\perp \perp}=U
$$

( $U$ tem dimensão finita e portanto é fechado). Por outro lado, $V \subseteq V+U^{\perp}$, assim

$$
\left(V+U^{\perp}\right)^{\perp} \subseteq V^{\perp} .
$$

Logo,

$$
\left(V+U^{\perp}\right)^{\perp} \subseteq V^{\perp} \cap U
$$

Dos fatos acima se segue

$$
\left(V+U^{\perp}\right)^{\perp}=V^{\perp} \cap U .
$$

Agora, sejam $P: V \rightarrow U$ a restrição a $V$ da projeção ortogonal sobre $U$ e $Q: U \rightarrow$ $V^{\perp} \cap U$ a restrição a $U$ da projeção ortogonal sobre $V^{\perp} \cap U$. Já que $V^{\perp} \cap U=\left(V+U^{\perp}\right)^{\perp}$, temos Ker $Q=U \cap\left(V+U^{\perp}\right)$. Dado que $V^{\perp} \cap U$ é subespaço de $U$, se segue que $Q$ é sobrejetora. Do Teorema do núcleo e da imagem temos

$$
\operatorname{dim} U=\operatorname{dim} \operatorname{Ker} Q+\operatorname{dim} \operatorname{Im} Q=\operatorname{dim}\left(U \cap\left(V+U^{\perp}\right)\right)+\operatorname{dim}\left(V^{\perp} \cap U\right) .
$$


Tomemos $u \in U$ fixado. Se $u=P(v)$ para algum $v \in V$, então $u=v+(P(v)-v)$, onde $(P(v)-v) \in U^{\perp}$. Consequentemente, $u \in U \cap\left(V+U^{\perp}\right)$ e assim $Q(u)=0$.

Reciprocamente, se $Q(u)=0$, temos $u=v+a \operatorname{com} v \in V$ e $a \in U^{\perp}$. Daí,

$$
u=P(u)=P(v+a)=P(v)+P(a)=P(v) .
$$

Os dois fatos acima provam que

$$
\operatorname{Im} P=U \cap\left(V+U^{\perp}\right)=\operatorname{Ker} Q .
$$

É claro que Ker $P=V \cap U^{\perp}$. Logo, pelo Teorema do núcleo e da imagem,

$$
\operatorname{dim} V=\operatorname{dim} \operatorname{Ker} P+\operatorname{dim} \operatorname{Im} P=\operatorname{dim}\left(V \cap U^{\perp}\right)+\operatorname{dim} \operatorname{Ker} Q .
$$

Portanto, de (4.4.10) e (4.4.11) temos

$$
\begin{aligned}
\operatorname{dim} U-\operatorname{dim} V & =\operatorname{dim}\left(V^{\perp} \cap U\right)+\operatorname{dim} \operatorname{Ker} Q-\operatorname{dim}\left(V \cap U^{\perp}\right)-\operatorname{dim} \operatorname{Ker} Q \\
& =\operatorname{dim}\left(V^{\perp} \cap U\right)-\operatorname{dim}\left(V \cap U^{\perp}\right),
\end{aligned}
$$

o que prova o lema.

Proposição 4.4.9. Se $L, T \in L(H)$ são isomorfismos essencialmente positivos e congruentes, então

$$
\mu_{r e l}(L, T)=\mu(L)-\mu(T) .
$$

Demonstração. Dado que, por hipótese, $H_{-}(L)$ e $H_{-}(T)$ têm dimensão finita, do lema anterior se segue que

$$
\operatorname{dim}\left(H_{-}(L)\right)-\operatorname{dim}\left(H_{-}(T)\right)=\operatorname{dim}\left[H_{-}(L) \cap\left(H_{-}(T)\right)^{\perp}\right]-\operatorname{dim}\left[H_{-}(T) \cap\left(H_{-}(L)\right)^{\perp}\right],
$$

isto é,

$$
\mu(L)-\mu(T)=\operatorname{dim}\left[H_{-}(L) \cap\left(H_{-}(T)\right)^{\perp}\right]-\operatorname{dim}\left[H_{-}(T) \cap\left(H_{-}(L)\right)^{\perp}\right] .
$$

Assim, como consequência da definição do índice de Morse relativo obtemos

$$
\mu_{\text {rel }}(L, T)=\operatorname{dim}\left[H_{-}(L) \cap\left(H_{-}(T)\right)^{\perp}\right]-\operatorname{dim}\left[H_{-}(T) \cap\left(H_{-}(L)\right)^{\perp}\right]=\mu(L)-\mu(T),
$$

o que prova a proposição.

Observação 4.4.10. Se $H$ tem dimensão finita e $(L, T)$ é um par de isomorfismos auto-adjuntos, de forma análoga à prova da proposição anterior, obtemos que

$$
\mu_{r e l}(L, T)=\mu(L)-\mu(T) .
$$


Demonstraremos agora algumas outras propriedades que possui o índice de Morse relativo para pares de isomorfismos auto-adjuntos. Para este fim, vejamos primeiro o seguinte resultado.

Lema 4.4.11. Se $L \in \Phi_{S}(H)$ e $K \in K_{S}(H)$, então $L+K$ pertence à mesma componente conexa de $\Phi_{S}(H)$ à qual pertence $L$.

Demonstração. Primeiramente, observe que a aplicação $\alpha:[0,1] \rightarrow \Phi_{S}(H)$ definida por $\alpha(t)=L+t K$ é contínua. Assim, sua imagem, $\alpha([0,1])$, é conexa.

Consideremos primeiro que $L \in \Phi_{S}^{+}(H)$. Suponhamos por absurdo que $L+K \notin$ $\Phi_{S}^{+}(H)$. Então, $L+K \in \Phi_{S}^{-}(H) \cup \Phi_{S}^{i}(H)$. Consequentemente,

$$
U=\alpha([0,1]) \cap \Phi_{S}^{+}(H) \quad \text { e } \quad V=\alpha([0,1]) \cap\left[\Phi_{S}^{-}(H) \cup \Phi_{S}^{i}(H)\right]
$$

são subconjuntos disjuntos não vazios cuja união é igual a $\alpha([0,1])$. Observe que $U$ e $V$ são subconjuntos abertos da imagem de $\alpha\left(\Phi_{S}^{+}(H), \Phi_{S}^{-}(H)\right.$ e $\Phi_{S}^{i}(H)$ são abertos de $\left.\Phi_{S}(H)\right)$, o que contradiz o fato de que $\alpha([0,1])$ é conexo. Em conclusão, $L+K \in \Phi_{S}^{+}(H)$.

De forma análoga podemos provar o caso em que $L$ pertence às outras duas componentes conexas de $\Phi_{S}(H)$.

Suponhamos que $L \in G L_{S}^{+}(H), K \in K_{S}(H)$ e que $L+K \in G L_{S}(H)$. Do Lema 4.4.11 obtemos que $\mu(L+K)<\infty$. Assim, dado que $\mu(L)=0$, a Proposição 4.4.9 implica que

$$
\mu_{\text {rel }}(L+K, L)=\mu(L+K) .
$$

A seguinte proposição é uma consequência do fato de que o índice de um caminho de pares de Fredholm é constante (Proposição 4.4.5).

Proposição 4.4.12. Se $\left\{\left(L_{t}, T_{t}\right)\right\}_{t \in J}$ é um caminho de pares de isomorfismos autoadjuntos congruentes, então $\mu_{\text {rel }}\left(L_{t}, T_{t}\right)$ é constante.

Demonstração. Como $\left\{\left(L_{t}, T_{t}\right)\right\}_{t \in J}$ é um caminho de pares de isomorfismos auto-adjuntos, então, se $t \in J, 0$ não pertence a os espectros dos operadores $L_{t}$ e $T_{t}$. Como foi visto na prova do Teorema 4.3.22, existe uma curva fechada simples $\Gamma$ em $\mathbb{C}$ tal que no seu interior ficam os espectros negativos dos operadores $L_{t}$ e $T_{t}$, para todo $t \in J$, e no seu exterior ficam os espectros positivos. Daí, as representações integrais das projeções ortogonais dos operadores $L_{t}$ e $T_{t}$ sobre seus subespaços espectrais negativos são dadas por

$$
P_{H_{-}\left(L_{t}\right)}=-\frac{1}{2 \pi i} \int_{\Gamma}\left(L_{t}-\lambda I\right)^{-1} d \lambda \quad \text { e } \quad P_{H_{-}\left(T_{t}\right)}=-\frac{1}{2 \pi i} \int_{\Gamma}\left(T_{t}-\lambda I\right)^{-1} d \lambda,
$$

para $t \in J$, respectivamente. Do Teorema 4.3.22 se segue que $t \mapsto P_{H_{-}\left(L_{t}\right)}$ e $t \mapsto P_{H_{-}\left(T_{t}\right)}$ são contínuas. Portanto, pela Proposição 4.4.6,

$$
\left\{\left(P_{H_{-}\left(L_{t}\right)}, P_{H_{-}\left(T_{t}\right)}\right)\right\}_{t \in J}
$$


é um caminho de pares de projeções ortogonais congruentes. Da Proposição 4.4.5 temos que

$$
\mu_{\text {rel }}\left(L_{t}, T_{t}\right)=\operatorname{ind}\left(P_{H_{-}\left(L_{t}\right)}, P_{H_{-}\left(T_{t}\right)}\right)
$$

é constante, o que prova a proposição.

Provemos agora que, se $S \in L(H)$ é um isomorfismo auto-adjunto e $O \in L(H)$ é ortogonal, então

$$
O^{*} P_{H_{-}(S)} O=P_{H_{-}\left(O^{*} S O\right)} .
$$

De fato, vejamos primeiro que $O^{*}\left(H_{-}(S)\right)=H_{-}\left(O^{*} S O\right)$. Denotemos por $\widetilde{S}$ o operador $O^{*} S O$. Se $x \in H_{-}(S)$,

$$
\left\langle O^{*} S O\left(O^{*} x\right), O^{*} x\right\rangle=\left\langle O^{*} S x, O^{*} x\right\rangle=\langle S x, x\rangle \leq 0 .
$$

Assim, $\widetilde{S}$ é negativa em $O^{*}\left(H_{-}(S)\right)$. Além disso,

$$
\widetilde{S}\left(O^{*}\left(H_{-}(S)\right)\right)=O^{*} S O\left(O^{*}\left(H_{-}(S)\right)\right)=O^{*} S\left(H_{-}(S)\right)=O^{*}\left(H_{-}(S)\right) .
$$

Analogamente, $\widetilde{S}$ é positiva em $O^{*}\left(H_{+}(S)\right)$ e

$$
\widetilde{S}\left(O^{*}\left(H_{+}(S)\right)\right)=O^{*}\left(H_{+}(S)\right) .
$$

É fácil ver que $\operatorname{Ker} \widetilde{S}=O^{*}(\operatorname{Ker} S)$. Consequentemente, de (4.4.14) e (4.4.15) e do Teorema 3.3.19 se segue

$$
H_{-}(\widetilde{S})=O^{*}\left(H_{-}(S)\right) \quad \text { e } \quad H_{+}(\widetilde{S})=O^{*}\left(H_{+}(S)\right) .
$$

Por outro lado, dado que $P_{H_{-}(S)}(H)=H_{-}(S)$, temos

$$
\operatorname{Im}\left(O^{*} P_{H_{-}(S)} O\right)=O^{*} P_{H_{-}(S)}(\operatorname{Im} O)=O^{*} P_{H_{-}(S)}(H)=O^{*}\left(H_{-}(S)\right) .
$$

Além disso, o operador $O^{*} P_{H_{-}(S)} O$ é uma projeção ortogonal, pois é auto-adjunto e $\left(O^{*} P_{H_{-}(S)} O\right)^{2}=O^{*} P_{H_{-}(S)} O$ (veja-se Proposição 3.2.13). Em conclusão, $O^{*} P_{H_{-}(S)} O$ é a projeção ortogonal sobre $O^{*}\left(H_{-}(S)\right)=H_{-}(\widetilde{S})$.

Uma consequência do Lema 4.4.3 e de (4.4.13) é dada na seguinte proposição.

Proposição 4.4.13. Seja $(L, T)$ um par de isomorfismos auto-adjuntos congruentes em $L(H)$. Se $O \in L(H)$ é ortogonal, então

$$
\mu_{r e l}(L, T)=\mu_{r e l}\left(O^{*} L O, O^{*} T O\right) .
$$


Demonstração. É claro que $O^{*} L O$ e $O^{*} T O$ são congruentes, pois

$$
O^{*} L O-O^{*} T O=O^{*}(L-T) O
$$

é compacto pelo Teorema 2.3.10. Por outro lado, do Lema 4.4.3 temos que

$$
\mu_{r e l}(L, T)=\operatorname{ind}\left(P_{H_{-}(L)}, P_{H_{-}(T)}\right)=\operatorname{ind}\left(O^{*} P_{H_{-}(L)} O, O^{*} P_{H_{-}(T)} O\right) .
$$

Da igualdade (4.4.13) se segue

$$
\begin{aligned}
\mu_{r e l}\left(O^{*} L O, O^{*} T O\right) & =\operatorname{ind}\left(P_{H_{-}\left(O^{*} L O\right)}, P_{H_{-}\left(O^{*} T O\right)}\right) \\
& =\operatorname{ind}\left(O^{*} P_{H_{-}(L)} O, O^{*} P_{H_{-}(T)} O\right) .
\end{aligned}
$$

Consequentemente, $\mu_{r e l}(L, T)=\mu_{\text {rel }}\left(O^{*} L O, O^{*} T O\right)$.

A seguinte proposição mostra um resultado mais geral que o fato anterior: o índice relativo de Morse relativo é invariante pela ação cogradiente.

Proposição 4.4.14. Sejam $L$ e $T$ em $L(H)$, isomorfismos auto-adjuntos e congruentes. Se $S \in G L(H)$, então $S^{*} L S$ e $S^{*} T S$ são congruentes e, além disso,

$$
\mu_{\text {rel }}\left(S^{*} L S, S^{*} T S\right)=\mu_{\text {rel }}(L, T) .
$$

Demonstração. Observe que $S^{*} L S$ e $S^{*} T S$ são congruentes, sendo $S^{*} L S-S^{*} T S$ compacto. Seja $S=O R$ a decomposição polar de $S$. Aqui, $O$ é ortogonal e $R$ denota a raiz quadrada não negativa do operador $S^{*} S$ (ver Teorema 3.3.16). Já que $R$ e $I$ são isomorfismos definidos positivos, do Corolário 3.4.8 temos que, para cada $t \in[0,1]$, o operador $(1-t) R+t I$ é um isomorfismo definido positivo. Definamos

$$
S_{t}=O((1-t) R+t I) \text { para } t \in[0,1] .
$$

É fácil ver diretamente da definição que $\left\{\left(S_{t}^{*} L S_{t}, S_{t}^{*} T S_{t}\right)\right\}_{t \in[0,1]}$ é um caminho de pares de isomorfismos auto-adjuntos congruentes. Assim, das Proposições 4.4 .12 e 4.4.13 temos

$$
\begin{aligned}
\mu_{r e l}\left(S^{*} L S, S^{*} T S\right) & =\mu_{r e l}\left(S_{0}^{*} L S_{0}, S_{0}^{*} T S_{0}\right)=\mu_{r e l}\left(S_{1}^{*} L S_{1}, S_{1}^{*} T S_{1}\right) \\
& =\mu_{r e l}\left(O^{*} L O, O^{*} T O\right)=\mu_{r e l}(L, T),
\end{aligned}
$$

o que prova a proposição.

No seguinte lema vamos supor que $L_{1}, T_{1} \in L\left(H_{1}\right)$ e $L_{2}, T_{2} \in L\left(H_{2}\right)$, onde $H_{1}$ e $H_{2}$ são espaços de Hilbert e, além disso, para evitar confusão, denotaremos por $\left[L_{1}, L_{2}\right] \in L\left(H_{1} \times H_{2}\right)$ e $\left[T_{1}, T_{2}\right] \in L\left(H_{1} \times H_{2}\right)$ os produtos diretos dos operadores envolvidos. 
Lema 4.4.15. Se $\left(L_{1}, T_{1}\right)$ e $\left(L_{2}, T_{2}\right)$ são pares de isomorfismos congruentes, então $\left(\left[L_{1}, L_{2}\right],\left[T_{1}, T_{2}\right]\right)$ é um par de isomorfismos congruentes e, além disso,

$$
\mu_{\text {rel }}\left(\left[L_{1}, L_{2}\right],\left[T_{1}, T_{2}\right]\right)=\mu_{\text {rel }}\left(L_{1}, T_{1}\right)+\mu_{\text {rel }}\left(L_{2}, T_{2}\right) .
$$

Demonstração. O operador $\left[L_{1}, L_{2}\right]-\left[T_{1}, T_{2}\right]=\left[L_{1}-T_{1}, L_{2}-T_{2}\right]$ é compacto pela Proposição 2.3.8. É claro que $\left[L_{1}, L_{2}\right]$ e $\left[T_{1}, T_{2}\right]$ são isomorfismos. Daí, $\left(\left[L_{1}, L_{2}\right],\left[T_{1}, T_{2}\right]\right)$ é um par de isomorfismos congruentes.

Por outro lado, o Lema 4.2.9 implica que

$$
H_{ \pm}\left[L_{1}, L_{2}\right]=H_{ \pm}\left(L_{1}\right) \times H_{ \pm}\left(L_{2}\right) \quad \text { e } \quad H_{ \pm}\left[T_{1}, T_{2}\right]=H_{ \pm}\left(T_{1}\right) \times H_{ \pm}\left(T_{2}\right) .
$$

Assim,

$$
\begin{aligned}
\mu_{\text {rel }}\left(\left[L_{1}, L_{2}\right],\left[T_{1}, T_{2}\right]\right)= & \operatorname{ind}\left(P_{H_{-}\left[L_{1}, L_{2}\right]}, P_{H_{-}\left[T_{1}, T_{2}\right]}\right) \\
= & \operatorname{dim}\left(\operatorname{Im} P_{H_{-}\left[L_{1}, L_{2}\right]} \cap \operatorname{Ker} P_{H_{-}\left[T_{1}, T_{2}\right]}\right) \\
& -\operatorname{dim}\left(\operatorname{Im} P_{H_{-}\left[T_{1}, T_{2}\right]} \cap \operatorname{Ker} P_{H_{-}\left[L_{1}, L_{2}\right]}\right) \\
= & \operatorname{dim}\left(\left[H_{-}\left(L_{1}\right) \times H_{-}\left(L_{2}\right)\right] \cap\left[H_{+}\left(T_{1}\right) \times H_{+}\left(T_{2}\right)\right]\right) \\
& -\operatorname{dim}\left(\left[H_{-}\left(T_{1}\right) \times H_{-}\left(T_{2}\right)\right] \cap\left[H_{+}\left(L_{1}\right) \times H_{+}\left(L_{2}\right)\right]\right) \\
= & \operatorname{dim}\left(\left[H_{-}\left(L_{1}\right) \cap H_{+}\left(T_{1}\right)\right] \times\left[H_{-}\left(L_{2}\right) \cap H_{+}\left(T_{2}\right)\right]\right) \\
& -\operatorname{dim}\left(\left[H_{-}\left(T_{1}\right) \cap H_{+}\left(L_{1}\right)\right] \times\left[H_{-}\left(T_{2}\right) \cap H_{+}\left(L_{2}\right)\right]\right) \\
= & \operatorname{dim}\left(H_{-}\left(L_{1}\right) \cap H_{+}\left(T_{1}\right)\right)+\operatorname{dim}\left(H_{-}\left(L_{2}\right) \cap H_{+}\left(T_{2}\right)\right) \\
& -\left[\operatorname{dim}\left(H_{-}\left(T_{1}\right) \cap H_{+}\left(L_{1}\right)\right)+\operatorname{dim}\left(H_{-}\left(T_{2}\right) \cap H_{+}\left(L_{2}\right)\right)\right] \\
= & {\left[\operatorname{dim}\left(H_{-}\left(L_{1}\right) \cap H_{+}\left(T_{1}\right)\right)-\operatorname{dim}\left(H_{-}\left(T_{1}\right) \cap H_{+}\left(L_{1}\right)\right)\right] } \\
& +\left[\operatorname{dim}\left(H_{-}\left(L_{2}\right) \cap H_{+}\left(T_{2}\right)\right)-\operatorname{dim}\left(H_{-}\left(T_{2}\right) \cap H_{+}\left(L_{2}\right)\right)\right] \\
= & \mu_{r e l}\left(L_{1}, T_{1}\right)+\mu_{r e l}\left(L_{2}, T_{2}\right) .
\end{aligned}
$$

Isto é, $\mu_{\text {rel }}\left(\left[L_{1}, L_{2}\right],\left[T_{1}, T_{2}\right]\right)=\mu_{r e l}\left(L_{1}, T_{1}\right)+\mu_{r e l}\left(L_{2}, T_{2}\right)$.

Finalizaremos este capítulo apresentando uma relação que liga o índice de Morse relativo com a assinatura generalizada de uma perturbação compacta auto-adjunta de uma simetria $\mathcal{J}$.

Proposição 4.4.16. Sejam $L_{a}=\mathcal{J}+K_{a}$ e $L_{b}=\mathcal{J}+K_{b}$ dois operadores inversíveis, onde $K_{a}$ e $K_{b}$ pertencem a $K_{S}(H)$. Então,

$$
\frac{1}{2}\left[\operatorname{sign}_{\mathcal{J}} L_{b}-\operatorname{sign}_{\mathcal{J}} L_{a}\right]=\mu_{\text {rel }}\left(L_{a}, L_{b}\right) .
$$

Demonstração. Sejam $\left(e_{i}^{ \pm}\right)_{i=1}^{\infty}$ bases de Hilbert de $H_{ \pm}(\mathcal{J})$ e

$$
H_{n}=\operatorname{span}\left\{e_{i}^{ \pm}: i=1, \ldots, n\right\} \quad \text { para } n \in \mathbb{N} .
$$


Denotemos por $P_{n}$ a projeção sobre $H_{n}$ para $n \in \mathbb{N}$. De forma análoga à prova da Proposição 4.2.5 podemos provar que existe um inteiro positivo $N$ tal que

$$
L_{\lambda, t, n}=\mathcal{J}+t K_{\lambda}+(1-t) P_{n} K_{\lambda} P_{n}: H \rightarrow H
$$

é injetor para todo $n \geq N, 0 \leq t \leq 1$ e $\lambda=a, b$. Além disso, dado que

$$
\left(P_{n} K_{\lambda} P_{n}\right)^{*}=P_{n}^{*} K_{\lambda}^{*} P_{n}^{*}=P_{n} K_{\lambda} P_{n}
$$

temos que $P_{n} K_{\lambda} P_{n}$ é auto-adjunto para $\lambda=a, b$ e $n \in \mathbb{N}$. Como $\mathcal{J}, K_{\lambda}$ e $P_{n} K_{\lambda} P_{n}$ são auto-adjuntos, para $\lambda=a, b$ e $n \in \mathbb{N}$, então $L_{a, t, n}$ e $L_{b, t, n}$ são auto-adjuntos para $t \in[0,1]$. Assim, $L_{\lambda, t, n}$ é um operador de Fredholm auto-adjunto para $\lambda=a, b$, e portanto ind $L_{\lambda, t, n}=0 . \operatorname{Logo}, \operatorname{Im} L_{\lambda, t, n}=H$, pois $\operatorname{Ker} L_{\lambda, t, n}=\{0\}$. Consequentemente, existe um inteiro positivo $N$ tal que $L_{\lambda, t, n}$ é um isomorfismo para todo $n \geq N, 0 \leq t \leq 1$ $\mathrm{e} \lambda=a, b$.

Se $n \geq N$, então $\left\{\left(L_{a, t, n}, L_{b, t, n}\right)\right\}_{t \in[0,1]}$ é um caminho de pares de isomorfismos autoadjuntos congruentes, pois

$$
L_{a, t, n}-L_{b, t, n}=t\left(K_{a}-K_{b}\right)+(1-t) P_{n}\left(K_{a}-K_{b}\right) P_{n}
$$

é compacto para todo $t \in[0,1]$. Assim, a Proposição 4.4 .12 prova que, se $n \geq N$,

$$
\mu_{r e l}\left(L_{a, 0, n}, L_{b, 0, n}\right)=\mu_{r e l}\left(L_{a, 1, n}, L_{b, 1, n}\right),
$$

isto é,

$$
\mu_{\text {rel }}\left(\mathcal{J}+P_{n} K_{a} P_{n}, \mathcal{J}+P_{n} K_{b} P_{n}\right)=\mu_{\text {rel }}\left(\mathcal{J}+K_{a}, \mathcal{J}+K_{b}\right) .
$$

Denotemos por $L_{\lambda, n}$ a $n$-ésima aproximação de Galerkin de $L_{\lambda}$ para $\lambda=a, b$. É claro que $H_{n}$ e $H_{n}^{\perp}$ são invariantes pelos operadores $\mathcal{J}+P_{n} K_{a} P_{n}$ e $\mathcal{J}+P_{n} K_{b} P_{n}$. Além disso, dado que $P_{n}$ é a projeção sobre $H_{n}$, temos que, se $y \in H_{n}^{\perp}$, então $P_{n} K_{a} P_{n} y=P_{n} K_{b} P_{n} y=0$. Este fato prova que

$$
\mathcal{J}+P_{n} K_{a} P_{n} \quad \text { e } \quad \mathcal{J}+P_{n} K_{b} P_{n} \quad \text { coincidem com } \mathcal{J} \text { em } H_{n}^{\perp} .
$$

É claro que $\mu_{\text {rel }}\left(\left.\mathcal{J}\right|_{H_{n}^{\perp}},\left.\mathcal{J}\right|_{H_{\frac{1}{n}}}\right)=0$. Assim, de (4.4.18) e do Lema 4.4.15 temos

$$
\begin{aligned}
& \mu_{\text {rel }}\left(\mathcal{J}+P_{n} K_{a} P_{n}, \mathcal{J}+P_{n} K_{b} P_{n}\right) \\
& \quad=\mu_{r e l}\left(\mathcal{J}+\left.P_{n} K_{a} P_{n}\right|_{H_{n}}, \mathcal{J}+\left.P_{n} K_{b} P_{n}\right|_{H_{n}}\right)+\mu_{r e l}\left(\mathcal{J}+\left.P_{n} K_{a} P_{n}\right|_{H_{n}^{\perp}}, \mathcal{J}+\left.P_{n} K_{b} P_{n}\right|_{H_{n}^{\perp}}\right) \\
& \quad=\mu_{r e l}\left(\mathcal{J}+\left.P_{n} K_{a} P_{n}\right|_{H_{n}}, \mathcal{J}+\left.P_{n} K_{b} P_{n}\right|_{H_{n}}\right)+\mu_{r e l}\left(\left.\mathcal{J}\right|_{H_{n}^{\perp}},\left.\mathcal{J}\right|_{H_{n}^{\perp}}\right) \\
& \quad=\mu_{r e l}\left(\mathcal{J}+\left.P_{n} K_{a} P_{n}\right|_{H_{n}}, \mathcal{J}+\left.P_{n} K_{b} P_{n}\right|_{H_{n}}\right) \\
& \quad=\mu_{r e l}\left(L_{a, n}, L_{b, n}\right) .
\end{aligned}
$$


Logo,

$$
\mu_{\text {rel }}\left(\mathcal{J}+P_{n} K_{a} P_{n}, \mathcal{J}+P_{n} K_{b} P_{n}\right)=\mu_{\text {rel }}\left(L_{a, n}, L_{b, n}\right) .
$$

Portanto, de (4.4.17) e (4.4.19) temos que, se $n \geq N$, então

$$
\mu_{r e l}\left(L_{a}, L_{b}\right)=\mu_{r e l}\left(L_{a, n}, L_{b, n}\right) .
$$

Por outro lado, pela definição da assinatura generalizada como o limite das assinaturas das aproximações de Galerkin, existe um inteiro positivo, que podemos seguir chamando de $N$, tal que

$$
\operatorname{sign}_{\mathcal{J}} L_{\lambda}=\operatorname{sign} L_{\lambda, n} \text { para } n \geq N, \lambda=a, b .
$$

Consequentemente, de (4.4.20) se segue que a igualdade em (4.4.16) será provada se mostrarmos que

$$
\frac{1}{2}\left[\operatorname{sign} L_{b, n}-\operatorname{sign} L_{a, n}\right]=\mu_{r e l}\left(L_{a, n}, L_{b, n}\right) \quad \text { para } n \geq N .
$$

Para provar a igualdade acima, observe primeiro que

$$
\mu\left(L_{a, n}\right)+\mu\left(-L_{a, n}\right)=\operatorname{dim} H_{n}=2 n=\mu\left(L_{b, n}\right)+\mu\left(-L_{b, n}\right) .
$$

Assim,

$$
\begin{aligned}
\frac{1}{2}\left[\operatorname{sign} L_{b, n}-\operatorname{sign} L_{a, n}\right] & =\frac{1}{2}\left[\mu\left(-L_{b, n}\right)-\mu\left(L_{b, n}\right)-\left(\mu\left(-L_{a, n}\right)-\mu\left(L_{a, n}\right)\right)\right] \\
& =\frac{1}{2}\left[2 n-\mu\left(L_{b, n}\right)-\mu\left(L_{b, n}\right)-\left(2 n-\mu\left(L_{a, n}\right)-\mu\left(L_{a, n}\right)\right)\right] \\
& =\frac{1}{2}\left[-2 \mu\left(L_{b, n}\right)+2 \mu\left(L_{a, n}\right)\right] \\
& =\mu\left(L_{a, n}\right)-\mu\left(L_{b, n}\right),
\end{aligned}
$$

isto é,

$$
\frac{1}{2}\left[\operatorname{sign} L_{b, n}-\operatorname{sign} L_{a, n}\right]=\mu\left(L_{a, n}\right)-\mu\left(L_{b, n}\right) .
$$

Pelo lema anterior temos

$$
\begin{aligned}
\mu\left(L_{a, n}\right)-\mu\left(L_{b, n}\right) & =\operatorname{dim}\left(H_{-}\left(L_{a, n}\right)\right)-\operatorname{dim}\left(H_{-}\left(L_{b, n}\right)\right) \\
& =\operatorname{dim}\left(H_{-}\left(L_{a, n}\right) \cap\left(H_{-}\left(L_{b, n}\right)\right)^{\perp}\right)-\operatorname{dim}\left(H_{-}\left(L_{b, n}\right) \cap\left(H_{-}\left(L_{a, n}\right)\right)^{\perp}\right) \\
& =\operatorname{ind}\left(P_{H_{-}\left(L_{a, n}\right)}, P_{H_{-}\left(L_{b, n}\right)}\right) \\
& =\mu_{r e l}\left(L_{a, n}, L_{b, n}\right) .
\end{aligned}
$$

Se segue de (4.4.21) e do fato anterior que

$$
\mu_{r e l}\left(L_{a, n}, L_{b, n}\right)=\frac{1}{2}\left[\operatorname{sign} L_{b, n}-\operatorname{sign} L_{a, n}\right],
$$

o que prova a proposição. 
Como consequência temos que, se $K \in K_{S}(H)$ e $\mathcal{J}+K$ é um isomorfismo, então

$$
\mu_{\text {rel }}(\mathcal{J}, \mathcal{J}+K)=\frac{1}{2}\left[\operatorname{sign}_{\mathcal{J}}(\mathcal{J}+K)-\operatorname{sign}_{\mathcal{J}}(\mathcal{J})\right]=\frac{1}{2}\left[\operatorname{sign}_{\mathcal{J}}(\mathcal{J}+K)\right]
$$




\section{Capítulo 5}

\section{O fluxo espectral de caminhos de operadores de Fredholm auto-adjuntos}

Neste capítulo vamos construir o fluxo espectral para caminhos de operadores de Fredholm auto-adjuntos e, além disso, veremos algumas das suas propriedades. Para este fim, será introduzido, na próxima seção, o conceito de parametrix cogradiente para uma família de operadores fortemente indefinidos, e usaremos a noção de assinatura generalizada, apresentada no capítulo anterior.

Na segunda seção o fluxo espectral será definido primeiramente para caminhos em $\Phi_{S}^{i}(H)$ com extremos inversíveis. Veremos que esta noção se pode estender a caminhos fechados em $\Phi_{S}^{i}(H)$, onde seus extremos não necessariamente são inversíveis. Mostraremos que o fluxo espectral é invariante por ação cogradiente e também por homotopias.

$\mathrm{Na}$ Seção 3 abordaremos o caso para caminhos com valores nas outras duas componentes conexas de $\Phi_{S}(H)$, ou seja, em $\Phi_{S}^{+}(H)$ e em $\Phi_{S}^{-}(H)$. Veremos que, se $\mathrm{L}=\left\{\mathrm{L}_{\lambda}\right\}_{\lambda \in J}$ é um caminho de operadores essencialmente positivos com extremos inversíveis, então o fluxo espectral de $\mathrm{L}$ é igual à diferença

$$
\mu\left(\mathrm{L}_{a}\right)-\mu\left(\mathrm{L}_{b}\right)
$$

Finalizaremos o trabalho na quarta seção, onde daremos a definição do fluxo espectral em pontos singulares isolados de um caminho de operadores de Fredholm autoadjuntos. O propósito desta seção é mostrar que, se $L=\left\{L_{\lambda}\right\}_{\lambda \in J}$ é um caminho continuamente diferenciável de operadores de Fredholm auto-adjuntos e $\lambda_{0}$ é um ponto singular isolado de $L$, então o fluxo espectral de $L$ em $\lambda_{0}$ coincide com a assinatura do diferencial $\dot{L}_{\lambda_{0}}$ restrito ao núcleo de $L_{\lambda_{0}}$. 


\subsection{Parametrix cogradiente}

O objetivo desta seção é mostrar que toda família de operadores de Fredholm autoadjuntos fortemente indefinidos, parametrizada por um espaço topológico paracompacto e contrátil, possui uma parametrix cogradiente, que definiremos mais adiante. Como premissa para a prova deste fato, primeiro lembraremos algumas noções básicas da análise funcional, da teoria dos grupos e da topologia algébrica.

Nesta seção $H$ denotará um espaço de Hilbert real, separável e de dimensão infinita.

Definição 5.1.1 (Forma bilinear). Sejam $H_{1}$ e $H_{2}$ espaços de Hilbert reais. Uma forma bilinear é uma aplicação

$$
h: H_{1} \times H_{2} \rightarrow \mathbb{R}
$$

tal que, para todo $x, x_{1}, x_{2} \in H_{1}, y, y_{1}, y_{2} \in H_{2}$ e $a, b \in \mathbb{R}$,

i. $h\left(a x_{1}+b x_{2}, y\right)=a h\left(x_{1}, y\right)+b h\left(x_{2}, y\right) \mathrm{e}$

ii. $h\left(x, a y_{1}+b y_{2}\right)=a h\left(x, y_{1}\right)+b h\left(x, y_{2}\right)$.

Se $H_{1}=H_{2}$ e $h(x, y)=h(y, x)$ para todo $x, y \in H_{1}$ dizemos que $h$ é simétrica.

Uma forma bilinear $h: H_{1} \times H_{2} \rightarrow \mathbb{R}$ é limitada se existe $c>0$ tal que

$$
|h(x, y)| \leq c\|x\|\|y\| \quad \text { para todo }(x, y) \in H_{1} \times H_{2} .
$$

Neste caso, o número

$$
\|h\|=\sup _{\substack{\|x\|=1, x \in H_{1} \\\|y\|=1, y \in H_{2}}}|h(x, y)|
$$

é chamado de norma de $h$.

O seguinte teorema, cuja prova se pode ver, por exemplo, em [16], pág. 192, Teorema 3.8-4, é o bem conhecido Teorema da representação de Riesz para formas bilineares.

Teorema 5.1.2 (Teorema da representação de Riesz). Sejam $H_{1}, H_{2}$ espaços de Hilbert reais e $h: H_{1} \times H_{2} \rightarrow \mathbb{R}$ uma forma bilinear limitada. Então, $h$ tem uma representação

$$
h(x, y)=\langle S x, y\rangle
$$

onde $S: H_{1} \rightarrow H_{2}$ é um operador linear limitado, unicamente determinado por $h$, $e$

$$
\|h\|=\|S\| .
$$

Observação 5.1.3. Nas condições do teorema anterior, é claro que, se $H_{1}=H_{2}$ e $h: H_{1} \times H_{1} \rightarrow \mathbb{R}$ é simétrica, então $S$ é auto-adjunto. 
Definição 5.1.4 (G-ação). Sejam $(G, \cdot)$ um grupo e $X$ um conjunto. Uma $G$-ação sobre $X$ é uma aplicação $\alpha: G \times X \rightarrow X$ com as seguintes propriedades;

i. Para todo $x \in X, \alpha(e, x)=x$, onde $e$ é o elemento neutro de $G$.

ii. Para todo $x \in X, g, h \in G, \alpha(g \cdot h, x)=\alpha(g, \alpha(h, x))$.

Neste caso dizemos que $X$ é um G-grupo.

Se $g \in G$ e $x \in X$, o elemento $\alpha(g, x)$ será denotado por $\alpha_{g}(x)$. A definição acima implica que, para cada $g \in G$, a aplicação $\alpha_{g}: X \rightarrow X$, que podemos, se necessário, denotar por $\alpha_{g}(x)=g x$ para $x \in X$, possui a inversa $\alpha_{g^{-1}}: X \rightarrow X$, definida por $\alpha_{g^{-1}}(x)=g^{-1} x$ para $x \in X$. Portanto, se $G$ é um grupo topológico e $X$ é um espaço topológico, para cada $g \in G$, a aplicação $\alpha_{g}$ é um homeomorfismo. É claro que todo grupo $G$ é um $G$-grupo.

Definição 5.1.5. Sejam $G$ um grupo e $X, Y$ dois $G$-grupos com $G$-ações $\alpha$ e $\beta$, respectivamente. Uma aplicação $\psi: X \rightarrow Y$ é G-equivariante se

$$
\psi\left(\alpha_{g}(x)\right)=\beta_{g}(\psi(x)) \text { para todo } x \in X \text { e } g \in G \text {. }
$$

Neste caso dizemos que $\psi$ é equivariante entre os $G$-grupos $X$ e $Y$.

Definição 5.1.6 (Fibrado). Sejam $X, B$ e $F$ três espaços topológicos. Uma aplicação contínua e sobrejetora $\pi: X \rightarrow B$ é um fibrado trivial com fibra $F$ e base $B$ se existe um homeomorfismo $\phi: X \rightarrow B \times F$ tal que

$$
\pi(x)=\pi_{1} \phi(x) \quad \text { para todo } x \in X,
$$

onde $\pi_{1}: B \times F \rightarrow B$ é a projeção sobre a primeira coordenada.

Mais geralmente, dizemos que $\pi: X \rightarrow B$ é um fibrado localmente trivial com fibra $F$ e base $B$ se para qualquer ponto $b \in B$ existe uma vizinhança $U$ de $b$ e um homeomorfismo $\phi_{U}: \pi^{-1}(U) \rightarrow U \times F$ tal que

$$
\pi(x)=\pi_{1} \phi_{U}(x) \quad \text { para todo } x \in \pi^{-1}(U) .
$$

A aplicação $\phi_{U}$ é chamada uma trivialização sobre a vizinhança $U$ de $b$.

Sejam $B^{\prime}$ um espaço topológico e $f: B^{\prime} \rightarrow B$ uma aplicação contínua. O pullback induzido por $f$ e pelo fibrado $\pi: X \rightarrow B$ é a aplicação $f^{\star}(\pi): X^{\prime} \rightarrow B^{\prime}$, onde $X^{\prime}$ é o subespaço do produto $B^{\prime} \times X$ consistente dos pares $\left(b^{\prime}, x\right)$ para o qual $f\left(b^{\prime}\right)=\pi(x)$, e $f^{\star}(\pi)$ é a aplicação definida pela projeção $\left(b^{\prime}, x\right) \mapsto b^{\prime}$ para todo $\left(b^{\prime}, x\right) \in X^{\prime}$.

Os dois seguintes resultados, cujas provas se podem ver, por exemplo, em [23], pág. 61, Lema 1.3 e pág. 75, Proposição 3.5, respectivamente, serão fundamentais nesta seção. 
Lema 5.1.7. Sejam $\pi: X \rightarrow B$ um fibrado localmente trivial com fibra $F$ e $f: B^{\prime} \rightarrow B$ uma aplicação contínua. Então, o pullback induzido por $f$ e $\pi, f^{\star}(\pi): X^{\prime} \rightarrow B^{\prime}$, é também um fibrado localmente trivial com fibra $F$ e base $B^{\prime}$.

Proposição 5.1.8. Seja $\pi: X \rightarrow B$ um fibrado localmente trivial com fibra $F$. Se $B$ é paracompacto e contrátil, então $\pi$ é um fibrado trivial.

Definição 5.1.9 (Parametrix cogradiente). Sejam $\Lambda$ um espaço topológico e $\mathrm{L}=$ $\left\{\mathrm{L}_{\lambda}\right\}_{\lambda \in \Lambda}$ uma família de operadores de Fredholm auto-adjuntos fortemente indefinidos, isto é, uma aplicação contínua $\mathrm{L}: \Lambda \rightarrow \Phi_{S}^{i}(H)$. Uma parametrix cogradiente para $\mathrm{L}$ relativa a uma simetria $\mathcal{J} \in \Phi_{S}^{i}(H)$ é uma família $\mathrm{M}=\left\{\mathrm{M}_{\lambda}\right\}_{\lambda \in \Lambda}$ de operadores em $G L(H)$ tal que, para cada $\lambda \in \Lambda$,

$$
\mathrm{M}_{\lambda}^{*} \mathrm{~L}_{\lambda} \mathrm{M}_{\lambda}=\mathcal{J}+\mathrm{K}_{\lambda}
$$

onde $\mathrm{K}_{\lambda} \in K_{S}(H)$. Neste caso diremos que as famílias L e M são cogradientes.

No final desta seção mostraremos que, se $\Lambda$ é paracompacto e contrátil, então uma família de operadores $L=\left\{L_{\lambda}\right\}_{\lambda \in \Lambda}$ possui uma parametrix cogradiente. Para este fim, primeiro veremos alguns resultados que serão usados nesta prova.

Tomemos $\mathcal{G}=G L(H) \times K_{S}(H)$ e $\Phi_{S}^{i}(H)$ como espaços topológicos com as topologias herdadas de $L(H) \times L(H)$ e de $L(H)$, respectivamente. Se segue da Proposição 3.4.11 que, se $M \in G L(H)$ e $L \in \Phi_{S}^{i}(H)$, então $M L M^{*} \in \Phi_{S}^{i}(H)$. Além disso, se $K \in K_{S}(H)$, do Lema 4.4.11 temos $M L M^{*}+K \in \Phi_{S}^{i}(H)$. Seja

$$
\begin{aligned}
\pi: \mathcal{G} & \rightarrow \Phi_{S}^{i}(H) \\
(M, K) & \mapsto M \mathcal{J} M^{*}+K
\end{aligned}
$$

Dos Teoremas 3.2.2 e 3.2.3 se segue que o operador que assina a cada $L \in L(H)$ seu adjunto $L^{*}$ é um operador linear limitado. Portanto, $\pi$ é contínua, sendo soma e composição de aplicações contínuas.

Mostraremos que $\pi$ é um fibrado localmente trivial com fibra $\pi^{-1}(\mathcal{J})$. Para este fim, primeiro provemos que $\mathcal{G}$ pode ser dotado com uma estrutura de grupo topológico.

Proposição 5.1.10. A operação

$$
g \cdot g^{\prime}=\left(M M^{\prime}, K+M K^{\prime} M^{*}\right), \quad \text { para } g=(M, K), g^{\prime}=\left(M^{\prime}, K^{\prime}\right) \in \mathcal{G},
$$

define uma estrutura de grupo topológico para o conjunto $\mathcal{G}$.

Demonstração. De fato, $g \cdot g^{\prime} \in \mathcal{G}$, pois $M M^{\prime} \in G L(H)$ e $K+M K^{\prime} M^{*} \in K_{S}(H)$. É fácil ver que o elemento neutro de $\mathcal{G}$ é $(I, 0)$, onde $I$ é a identidade em $G L(H)$ e 0 é o operador nulo. Por outro lado, se $g=(M, K) \in \mathcal{G}$, então $g^{-1}=\left(M^{-1},-M^{-1} K\left(M^{-1}\right)^{*}\right)$, pois

$$
(M, K) \cdot\left(M^{-1},-M^{-1} K\left(M^{-1}\right)^{*}\right)=\left(M M^{-1}, K+M\left(-M^{-1} K\left(M^{-1}\right)^{*}\right) M^{*}\right)=(I, 0)
$$


e

$\left(M^{-1},-M^{-1} K\left(M^{-1}\right)^{*}\right) \cdot(M, K)=\left(M^{-1} M,-M^{-1} K\left(M^{-1}\right)^{*}+M^{-1} K\left(M^{-1}\right)^{*}\right)=(I, 0)$.

É claro que $g^{-1} \in \mathcal{G}$. Por último vejamos que o produto dado em (5.1.2) é associativo. Se $\left(M_{1}, K_{1}\right),\left(M_{2}, K_{2}\right)$ e $\left(M_{3}, K_{3}\right)$ são elementos de $\mathcal{G}$, então

$$
\begin{aligned}
\left(\left(M_{1}, K_{1}\right) \cdot\left(M_{2}, K_{2}\right)\right) \cdot\left(M_{3}, K_{3}\right) & =\left(M_{1} M_{2}, K_{1}+M_{1} K_{2} M_{1}^{*}\right) \cdot\left(M_{3}, K_{3}\right) \\
& =\left(M_{1} M_{2} M_{3}, K_{1}+M_{1} K_{2} M_{1}^{*}+M_{1} M_{2} K_{3}\left(M_{1} M_{2}\right)^{*}\right) \\
& =\left(M_{1} M_{2} M_{3}, K_{1}+M_{1} K_{2} M_{1}^{*}+M_{1} M_{2} K_{3} M_{2}^{*} M_{1}^{*}\right) \\
& =\left(M_{1} M_{2} M_{3}, K_{1}+M_{1}\left(K_{2}+M_{2} K_{3} M_{2}^{*}\right) M_{1}^{*}\right) \\
& =\left(M_{1}, K_{1}\right) \cdot\left(M_{2} M_{3}, K_{2}+M_{2} K_{3} M_{2}^{*}\right) \\
& =\left(M_{1}, K_{1}\right) \cdot\left(\left(M_{2}, K_{2}\right) \cdot\left(M_{3}, K_{3}\right)\right) .
\end{aligned}
$$

Consequentemente, $(\mathcal{G}, \cdot)$ é um grupo.

Por outro lado, é claro que

$$
\varsigma: \mathcal{G} \times \mathcal{G} \rightarrow \mathcal{G} \quad \text { dada por } \varsigma\left(g, g^{\prime}\right)=g \cdot g^{\prime}
$$

é contínua. Pelo Lema 1.1.9, a aplicação $M \mapsto M^{-1}$, para $M \in G L(H)$, é contínua. Logo,

$$
g \mapsto g^{-1}=\left(M^{-1},-M^{-1} K\left(M^{-1}\right)^{*}\right), \quad \text { onde } g=(M, K) \in \mathcal{G},
$$

é também contínua. Portanto, $\mathcal{G}$ é um grupo topológico.

Agora vejamos que existe uma $\mathcal{G}$-ação sobre $\Phi_{S}^{i}(H)$ de tal modo que $\pi: \mathcal{G} \rightarrow \Phi_{S}^{i}(H)$ se torna uma aplicação equivariante entre $\mathcal{G}$ e $\Phi_{S}^{i}(H)$.

Proposição 5.1.11. A aplicação $\tau: \mathcal{G} \times \Phi_{S}^{i}(H) \rightarrow \Phi_{S}^{i}(H)$ definida por

$$
\tau_{g}(L)=M L M^{*}+K \quad \text { para } L \in \Phi_{S}^{i}(H) \text { e } g=(M, K) \in \mathcal{G}
$$

é uma $\mathcal{G}$-ação contínua sobre $\Phi_{S}^{i}(H)$. Além disso, $\pi$ é equivariante entre $\mathcal{G}$ e $\Phi_{S}^{i}(H)$.

Demonstração. É claro que $\tau$ é contínua. Provemos que $\tau$ é uma ação de $\mathcal{G}$ em $\Phi_{S}^{i}(H)$. De fato, denotando $\tau(g, L)=\tau_{g}(L)$, temos

$$
\tau((I, 0), L)=L \quad \text { para todo } L \in \Phi_{S}^{i}(H) .
$$

Agora, fixemos $g=(M, K), g^{\prime}=\left(M^{\prime}, K^{\prime}\right) \in \mathcal{G}$ e $L \in \Phi_{S}^{i}(H)$. Assim,

$$
\begin{aligned}
\tau\left((M, K) \cdot\left(M^{\prime}, K^{\prime}\right), L\right) & =\tau\left(\left(M M^{\prime}, K+M K^{\prime} M^{*}\right), L\right) \\
& =M M^{\prime} L\left(M M^{\prime}\right)^{*}+K+M K^{\prime} M^{*} \\
& =M M^{\prime} L\left(M^{\prime}\right)^{*} M^{*}+K+M K^{\prime} M^{*} \\
& =M\left(M^{\prime} L\left(M^{\prime}\right)^{*}+K^{\prime}\right) M^{*}+K \\
& =M\left(\tau\left(\left(M^{\prime}, K^{\prime}\right), L\right)\right) M^{*}+K \\
& =\tau\left((M, K), \tau\left(\left(M^{\prime}, K^{\prime}\right), L\right)\right) .
\end{aligned}
$$


Este fato prova que $\Phi_{S}^{i}(H)$ é um $\mathcal{G}$-grupo.

Por outro lado, denotando $\varsigma_{g}\left(g^{\prime}\right)=\varsigma\left(g, g^{\prime}\right)$, sendo $\varsigma$ definida na (5.1.3),

$$
\begin{aligned}
\pi \varsigma_{g}\left(g^{\prime}\right) & =\pi\left(g \cdot g^{\prime}\right)=\pi\left(\left(M M^{\prime}, K+M K^{\prime} M^{*}\right)\right)=M M^{\prime} \mathcal{J}\left(M M^{\prime}\right)^{*}+K+M K^{\prime} M^{*} \\
& =M M^{\prime} \mathcal{J}\left(M^{\prime}\right)^{*} M^{*}+K+M K^{\prime} M^{*}=M\left(M^{\prime} \mathcal{J} M^{\prime *}+K^{\prime}\right) M^{*}+K \\
& =M\left(\pi\left(M^{\prime}, K^{\prime}\right)\right) M^{*}+K=\tau_{(M, K)}\left(\pi\left(M^{\prime}, K^{\prime}\right)\right) \\
& =\tau_{g} \pi\left(g^{\prime}\right) .
\end{aligned}
$$

Daí,

$$
\pi \varsigma_{g}\left(g^{\prime}\right)=\pi\left((M, K) \cdot\left(M^{\prime}, K^{\prime}\right)\right)=M\left(\pi\left(M^{\prime}, K^{\prime}\right)\right) M^{*}+K=\tau_{g} \pi\left(g^{\prime}\right) .
$$

Consequentemente, $\pi$ é equivariante entre os $\mathcal{G}$-grupos $\mathcal{G}$ e $\Phi_{S}^{i}(H)$.

Provaremos que existe uma vizinhança $\mathcal{U}$ do operador $\mathcal{J}$ no espaço $\Phi_{S}^{i}(H)$ e uma aplicação contínua $\varrho: \mathcal{U} \rightarrow \mathcal{G}$ tal que $\pi \varrho=\left.I\right|_{\mathcal{U}}$, ou seja, $\pi \varrho(L)=L$ para todo $L \in \mathcal{U}$. Primeiro vejamos os seguintes resultados.

Tomemos $D=\{a+i b \in \mathbb{C}: a>0\}$ e seja

$$
\gamma: D \rightarrow \mathbb{C}, \quad \text { dada por } \lambda \mapsto \lambda^{1 / 2}=|\lambda|^{1 / 2} e^{\frac{i \operatorname{Arg} z}{2}},
$$

onde $\operatorname{Arg} z$ denota o argumento principal de $z$. Podemos ver, por exemplo, em [6], pág. 64, Exemplo 15, que $\gamma$ é regular. É claro que $\gamma(\lambda) \gamma(\lambda)=\lambda$ para topo $\lambda \in D$.

Lembremos que a raiz quadrada não negativa de um operador não negativo $L \in$ $L(H)$ é um operador não negativo $R \in L(H)$ tal que $R^{2}=L$. Como mostramos no Teorema 3.3.14, a raiz quadrada de um operador não negativo existe e é única. Usando a aplicação $\gamma$, definida acima, provaremos o seguinte lema.

Lema 5.1.12. A aplicação

$$
\begin{aligned}
\mathcal{R}: G L_{S}^{+}(H) & \rightarrow G L_{S}^{+}(H) \\
L & \mapsto L^{1 / 2}
\end{aligned}
$$

onde $L^{1 / 2}$ denota a raiz quadrada não negativa do operador $L \in G L_{S}^{+}(H)$, é contínua.

Demonstração. Tomemos $L \in G L_{S}^{+}(H)$ fixado. Para demonstrar a continuidade de $\mathcal{R}$, primeiro mostremos que existe uma vizinhança $\mathcal{U}$ de $L$ tal que, para todo $T \in \mathcal{U}$, o operador $T^{1 / 2}$ é igual a $\gamma(T)$, onde $\gamma: D \rightarrow \mathbb{C}$ é a aplicação definida na fórmula (5.1.5). Lembramos que $\gamma(T)$ é definida segundo a fórmula (4.3.1). Seja $c>0$ tal que $\|L x\| \geq c\|x\|$ para todo $x \in H$ (veja-se Proposição 1.1.10). De forma análoga à prova do Corolário 4.3.23 podemos ver que, para todo $T \in B(L, c / 2) \subseteq G L_{S}^{+}(H)$,

$$
\sigma(T) \subseteq[c / 2,\|L\|+c / 2]
$$


Consideremos a seguinte parametrização da circunferência em $\mathbb{C}$ de centro $(\|L\|, 0)$ e raio $\|L\|-\frac{1}{6} c$ :

$$
\Gamma(t)=(\|L\|, 0)+\left(\|L\|-\frac{1}{6} c\right) e^{2 \pi i t}, \text { para } t \in[0,1] .
$$

Daí, para cada $T \in B(L, c / 2), \sigma(T) \subseteq \stackrel{\Gamma}{\Gamma}$ e, além disso,

$$
\gamma(T)=-\frac{1}{2 \pi i} \int_{\Gamma} \lambda^{1 / 2}(T-\lambda I)^{-1} d \lambda .
$$

Mostremos que $\gamma(T)=T^{1 / 2}$. Para este fim, provaremos que $\gamma(T)^{2}=T$ e $\gamma(T)$ é não negativo. De fato, dado que $\gamma(\lambda) \gamma(\lambda)=\lambda$ para topo $\lambda \in D$, do Lema 4.3 .15 obtemos que $\gamma(T)^{2}=T$. Vejamos agora que $\gamma(T)$ é auto-adjunto. Para cada $n \in \mathbb{N}$, tomemos uma partição $P_{n}=\left\{\lambda_{0}, \lambda_{1}, \ldots, \lambda_{n}\right\}$ de $\Gamma$, com

$$
2\|L\|-c / 6=\lambda_{0}<\lambda_{1}<\lambda_{2}<\ldots<\lambda_{n}=\lambda_{0}
$$

e tais que, para cada $k=1, \ldots, n,\left|\lambda_{k}-\lambda_{k-1}\right| \rightarrow 0$ quando $n \rightarrow \infty$. Além disso, para $k=0,1, \ldots, n$, seja $\xi_{k}=\bar{\lambda}_{n-k}$. Assim, $\xi_{k} \in \Gamma$ e $\xi_{0}<\xi_{1}<\ldots<\xi_{n}=\xi_{0}$. Dados $x, y \in H$, temos

$$
\begin{aligned}
\left\langle\sum_{k=1}^{n} \lambda_{k}^{1 / 2}\left(\lambda_{k}-\lambda_{k-1}\right)\left(T-\lambda_{k} I\right)^{-1} x, y\right\rangle & =\left\langle x, \sum_{k=1}^{n} \bar{\lambda}_{k}^{1 / 2}\left(\bar{\lambda}_{k}-\bar{\lambda}_{k-1}\right)\left(T-\bar{\lambda}_{k} I\right)^{-1} y\right\rangle \\
& =\left\langle x, \sum_{k=1}^{n} \xi_{n-k}^{1 / 2}\left(\xi_{n-k}-\xi_{n-k+1}\right)\left(T-\xi_{n-k} I\right)^{-1} y\right\rangle \\
& =\left\langle x,-\sum_{k=1}^{n} \xi_{n-k}^{1 / 2}\left(\xi_{n-k+1}-\xi_{n-k}\right)\left(T-\xi_{n-k} I\right)^{-1} y\right\rangle \\
& =\left\langle x,-\sum_{j=1}^{n} \xi_{j-1}^{1 / 2}\left(\xi_{j}-\xi_{j-1}\right)\left(T-\xi_{j-1} I\right)^{-1} y\right\rangle .
\end{aligned}
$$

Portanto,

$$
\left\langle-\frac{1}{2 \pi i} \sum_{k=1}^{n} \lambda_{k}^{1 / 2}\left(\lambda_{k}-\lambda_{k-1}\right)\left(T-\lambda_{k} I\right)^{-1} x, y\right\rangle=\left\langle x,-\frac{1}{2 \pi i} \sum_{j=1}^{n} \xi_{j-1}^{1 / 2}\left(\xi_{j}-\xi_{j-1}\right)\left(T-\xi_{j-1} I\right)^{-1} y\right\rangle .
$$

Dado que

$$
\begin{aligned}
& \lim _{n \rightarrow \infty}-\frac{1}{2 \pi i} \sum_{k=1}^{n} \lambda_{k}^{1 / 2}\left(\lambda_{k}-\lambda_{k-1}\right)\left(T-\lambda_{k} I\right)^{-1}=\gamma(T) \\
&=\lim _{n \rightarrow \infty}-\frac{1}{2 \pi i} \sum_{j=1}^{n} \xi_{j-1}^{1 / 2}\left(\xi_{j}-\xi_{j-1}\right)\left(T-\xi_{j-1} I\right)^{-1},
\end{aligned}
$$


se segue que $\langle\gamma(T) x, y\rangle=\langle x, \gamma(T) y\rangle$ para todo $x, y \in H$. Este fato prova que $\gamma(T)$ é auto-adjunto.

Por último, mostremos que $\gamma(T)$ é não negativo. Do Lema 4.3 .16 obtemos que

$$
\sigma(\gamma(T))=\gamma(\sigma(T))
$$

Assim, como o espectro de $T$ é positivo, então o espectro de $\gamma(T)$ é positivo. Das equações (4.3.16) e (4.3.14) obtemos, respectivamente, que

$$
\max _{\lambda \in \sigma(\gamma(T))} \lambda=\sup _{\|x\|=1}\langle\gamma(T) x, x\rangle \quad \text { e } \min _{\lambda \in \sigma(\gamma(T))} \lambda=\inf _{\|x\|=1}\langle\gamma(T) x, x\rangle .
$$

Consequentemente, dado que $\min _{\lambda \in \sigma(\gamma(T))} \lambda \geq 0$, se segue que $\gamma(T)$ é não negativo.

Os fatos acima mostram que $\gamma(T)$ é a raiz quadrada não negativa do $T$.

Agora provemos a continuidade de $\mathcal{R}$. Tomemos $T \in B(L, c / 2)$. De (1.1.1) obtemos

$$
(L-\lambda I)^{-1}-(T-\lambda I)^{-1}=-(L-\lambda I)^{-1}(L-T)(T-\lambda I)^{-1} \quad \text { para } \lambda \in \Gamma .
$$

Assim,

$$
\begin{aligned}
\gamma(L)-\gamma(T) & =-\frac{1}{2 \pi i} \int_{\Gamma} \lambda^{1 / 2}(L-\lambda I)^{-1} d \lambda+\frac{1}{2 \pi i} \int_{\Gamma} \lambda^{1 / 2}(T-\lambda I)^{-1} d \lambda \\
& =-\frac{1}{2 \pi i} \int_{\Gamma} \lambda^{1 / 2}\left[(L-\lambda I)^{-1}-(T-\lambda I)^{-1}\right] d \lambda \\
& =\frac{1}{2 \pi i} \int_{\Gamma} \lambda^{1 / 2}(L-\lambda I)^{-1}(L-T)(T-\lambda I)^{-1} d \lambda .
\end{aligned}
$$

Como na prova lo Lema 1.1.9, existe $M>0$ tal que

$$
\left\|(T-\lambda I)^{-1}\right\| \leq M \quad \text { para } \lambda \in \Gamma \text { e } T \in B(L, c / 2) .
$$

Assim,

$$
\left\|\lambda^{1 / 2}(L-\lambda I)^{-1}(L-T)(T-\lambda I)^{-1}\right\| \leq m M^{2}\|L-T\|, \text { onde } m=\max _{\lambda \in \Gamma}\left|\lambda^{1 / 2}\right| .
$$

Logo, dado $\varepsilon>0$, se $\|L-T\|<\varepsilon$, então, pelo Lema 4.3.21,

$$
\|\mathcal{R}(L)-\mathcal{R}(T)\|=\|\gamma(L)-\gamma(T)\|<\frac{1}{2 \pi} m M^{2} l \varepsilon,
$$

onde $l$ é o comprimento de $\Gamma$. Consequentemente, $\mathcal{R}$ é contínua.

Lema 5.1.13. Existe uma vizinhança $\mathcal{U}$ do operador $\mathcal{J}$ no espaço $\Phi_{S}^{i}(H)$ e uma seção $\varrho: \mathcal{U} \rightarrow \mathcal{G}$ de $\pi$, isto é, uma aplicação contínua $\varrho$ tal que $\pi \varrho=\left.I\right|_{\mathcal{U}}$. 
Demonstração. Escolhamos uma vizinhança $\mathcal{U}$ de $\mathcal{J}$ em $\Phi_{S}^{i}(H)$ que consista de operadores inversíveis (isto é possível pois $\mathcal{J}$ é inversível e o conjunto de operadores inversíveis é aberto). Para cada $T \in \mathcal{U}$, sejam $P_{T}^{+}$e $P_{T}^{-}$as projeções ortogonais de $H$ sobre os subespaços espectrais $H_{+}(T)$ e $H_{-}(T)$, respectivamente. Do Corolário 4.3.23 segue-se que elas dependem continuamente de $T$ em $\mathcal{U}$. Denotemos por $H_{+}$o subespaço $H_{+}(\mathcal{J})$ e por $H_{-}$o subespaço $H_{-}(\mathcal{J})$.

Dividiremos o resto da prova em vários passos:

Passo 1: Provemos que podemos escolher $\mathcal{U}$ suficientemente pequeno tal que cada $T \in \mathcal{U}$ tenha a propriedade que as restrições $\left.P_{T}^{+}\right|_{H_{+}}: H_{+} \rightarrow H_{+}(T)$ e $\left.P_{T}^{-}\right|_{H_{-}}: H_{-} \rightarrow$ $H_{-}(T)$ sejam dois isomorfismos. Para este fim, tomemos $\mathcal{U}$ suficientemente pequeno tal que o operador $P: \mathcal{U} \rightarrow L(H)$, definido por

$$
P(T)=P_{T}^{+} P_{\mathcal{J}}^{+}+P_{T}^{-} P_{\mathcal{J}}^{-}: H \rightarrow H, \quad \text { para } T \in \mathcal{U},
$$

seja uma bijeção. Este fato é possível pois $P(\mathcal{J})=I$, as projeções dependem continuamente de $T$, portanto $P(T)$ depende continuamente de $T$, e o conjunto dos isomorfismos é aberto. Da sobrejetividade de $P$ temos

$$
\begin{aligned}
H_{+}(T) \oplus H_{-}(T) & =H=P(T)(H)=\left(P_{T}^{+} P_{\mathcal{J}}^{+}+P_{T}^{-} P_{\mathcal{J}}^{-}\right)(H) \\
& \subseteq P_{T}^{+} P_{\mathcal{J}}^{+}(H)+P_{T}^{-} P_{\mathcal{J}}^{-}(H)=P_{T}^{+}\left(H_{+}\right) \oplus P_{T}^{-}\left(H_{-}\right),
\end{aligned}
$$

isto é, $H=P_{T}^{+}\left(H_{+}\right) \oplus P_{T}^{-}\left(H_{-}\right)$. Consequentemente, como $P_{T}^{ \pm}\left(H_{ \pm}\right) \subseteq H_{ \pm}(T)$,

$$
P_{T}^{ \pm}\left(H_{ \pm}\right)=H_{ \pm}(T) .
$$

Da injetividade de $P$ se segue que $P_{T}^{ \pm}$leva $H_{ \pm}$injetivamente sobre $H_{ \pm}(T)$. Logo, se $T \in \mathcal{U}$, a restrição de $P_{T}^{ \pm}$a $H_{ \pm}$é um isomorfismo sobre $H_{ \pm}(T)$. Denotemos por $Q_{T}^{ \pm}: H_{ \pm} \rightarrow H_{ \pm}(T)$ tal restrição.

Passo 2: Existem duas aplicações contínuas $S^{+}: \mathcal{U} \rightarrow G L\left(H_{+}\right)$e $S^{-}: \mathcal{U} \rightarrow$ $G L\left(H_{-}\right)$tais que

$$
\left\langle S^{+}(T) P_{T}^{+} T P_{T}^{+} S^{+}(T) u, v\right\rangle=\langle u, v\rangle \quad \text { e } \quad\left\langle S^{-}(T) P_{T}^{-} T P_{T}^{-} S^{-}(T) w, z\right\rangle=-\langle w, z\rangle
$$

para todo $T \in \mathcal{U}, u, v \in H_{+}$e $w, z \in H_{-}$. De fato, para cada $T \in \mathcal{U}$ tomemos a forma bilinear $B_{+}(T)$ em $H_{+}$dada por

$$
B_{+}(T)(u, v)=\left\langle T Q_{T}^{+} u, Q_{T}^{+} v\right\rangle \quad \text { para } u, v \in H_{+} .
$$

Então, $B_{+}(T)$ é simétrica e limitada. Pelo Teorema da representação de Riesz (Teorema 5.1.2), $B_{+}(T)$ é representado pelo operador auto-adjunto $A(T)=Q_{T}^{+*} T Q_{T}^{+} \in L\left(H_{+}\right)$e

$$
\sup _{\substack{v \in H_{+},\|v\|=1 \\ u \in H_{+},\|u\|=1}}\left|B_{+}(T)(u, v)\right|=\sup _{u \in H_{+},\|u\|=1}\|A(T) u\| .
$$


Provemos que a aplicação $A: \mathcal{U} \rightarrow L\left(H_{+}\right)$que associa a cada operador $T \in \mathcal{U}$ o operador $A(T) \in L\left(H_{+}\right)$é contínua. Seja $T \in \mathcal{U}$ fixado. Já que $Q_{L}^{ \pm}=P_{L}^{ \pm}$para $L \in \mathcal{U}$, de (5.1.7), (5.1.8) e da desigualdade de Cauchy-Schwarz, se segue que, para $S \in \mathcal{U}$,

$$
\begin{aligned}
\|A(S)-A(T)\| & =\sup _{\substack{u \in H_{+},\|u\|=1\\
}}\|(A(S)-A(T)) u\| \\
& \sup _{\substack{v \in H_{+},\|v\|=1 \\
u \in H_{+},\|u\|=1}}\left|\left\langle\left(S P_{S}^{+}-T P_{T}^{+}\right) u,\left(P_{S}^{+}-P_{T}^{+}\right) v\right\rangle\right| \\
\leq & \sup _{\substack{v \in H_{+},\|v\|=1 \\
u \in H_{+},\|u\|=1}}\left\|\left(S P_{S}^{+}-T P_{T}^{+}\right) u\right\|\left\|\left(P_{S}^{+}-P_{T}^{+}\right) v\right\| \\
\leq & \left\|S P_{S}^{+}-T P_{T}^{+}\right\|\left\|P_{S}^{+}-P_{T}^{+}\right\| \\
& =\left\|(S-T)\left(P_{S}^{+}-P_{T}^{+}\right)+T\left(P_{S}^{+}-P_{T}^{+}\right)+(S-T) P_{T}^{+}\right\|\left\|P_{S}^{+}-P_{T}^{+}\right\| \\
& \leq\left[\|S-T\|\left\|P_{S}^{+}-P_{T}^{+}\right\|+\|T\|\left\|P_{S}^{+}-P_{T}^{+}\right\|+\|S-T\|\left\|P_{T}^{+}\right\|\right]\left\|P_{S}^{+}-P_{T}^{+}\right\| .
\end{aligned}
$$

Agora, seja $\varepsilon>0$ dado. Como as projeções dependem continuamente de $T$ e $S$, existe $\delta>0, \operatorname{com} \delta<\min \left\{\varepsilon^{1 / 4}, \varepsilon^{1 / 4} /\left\|P_{T}^{+}\right\|\right\}$, tal que, se $\|T-S\|<\delta$, então

$$
\left\|P_{T}^{+}-P_{S}^{+}\right\|<\min \left\{\varepsilon^{1 / 2}, \varepsilon^{1 / 4} / 3, \varepsilon^{1 / 4} /(3\|T\|), \sqrt{\varepsilon} /\left(3\left\|P_{T}^{+}\right\|\right)\right\} .
$$

Consequentemente, se $\|T-S\|<\delta$,

$$
\|A(T)-A(S)\|<\frac{1}{3}\left[\varepsilon^{1 / 4} \varepsilon^{1 / 4}+\varepsilon^{1 / 2}+\varepsilon^{1 / 4} \varepsilon^{1 / 4}\right] \varepsilon^{1 / 2}=\varepsilon,
$$

o que prova que $A$ é contínua.

Fixemos $T$ em $\mathcal{U}$. Observe que $A(T)$ é definido positivo. De fato, dado que $P_{T}^{+}$é injetor em $H_{+}$, se $u \in H_{+} \operatorname{com} u \neq 0$, então $P_{T}^{+} u \neq 0$. Assim, como $P_{T}^{+} u \in H_{+}(T)$,

$$
\langle A(T) u, u\rangle=\left\langle T Q_{T}^{+} u, Q_{T}^{+} u\right\rangle=\left\langle T P_{T}^{+} u, P_{T}^{+} u\right\rangle>0
$$

Não é difícil provar que $A(T)$ é um isomorfismo. Os fatos acima mostram que $A(T)$ é um isomorfismo definido positivo.

Seja $S_{T}^{+} \in L\left(H_{+}\right)$a raiz quadrada não negativa da inversa de $A(T)$, isto é,

$$
S_{T}^{+}=\left(A(T)^{-1}\right)^{1 / 2}
$$

É fácil ver que

$$
\left(S_{T}^{+}\right)^{-1}\left(S_{T}^{+}\right)^{-1}=A(T) .
$$

Como $S_{T}^{+}$é auto-adjunto, temos

$$
\left(\left(S_{T}^{+}\right)^{-1}\right)^{*}=\left(\left(S_{T}^{+}\right)^{*}\right)^{-1}=\left(S_{T}^{+}\right)^{-1} .
$$


Assim, para $u, v \in H_{+}$, de (5.1.7) e (5.1.8) obtemos

$$
\begin{aligned}
\langle u, v\rangle & =\left\langle\left(S_{T}^{+}\right)^{-1} S_{T}^{+} u,\left(S_{T}^{+}\right)^{-1} S_{T}^{+} v\right\rangle=\left\langle\left(\left(S_{T}^{+}\right)^{-1}\right)^{*}\left(S_{T}^{+}\right)^{-1} S_{T}^{+} u, S_{T}^{+} v\right\rangle \\
& =\left\langle\left(S_{T}^{+}\right)^{-1}\left(S_{T}^{+}\right)^{-1} S_{T}^{+} u, S_{T}^{+} v\right\rangle=\left\langle A(T) S_{T}^{+} u, S_{T}^{+} v\right\rangle \\
& =\left\langle T P_{T}^{+} S_{T}^{+} u, P_{T}^{+} S_{T}^{+} v\right\rangle=\left\langle S_{T}^{+} P_{T}^{+} T P_{T}^{+} S_{T}^{+} u, v\right\rangle .
\end{aligned}
$$

Portanto, a aplicação

$$
\begin{aligned}
S^{+}: \mathcal{U} & \rightarrow G L\left(H_{+}\right) \\
T & \mapsto S^{+}(T)=S_{T}^{+}
\end{aligned}
$$

é tal que, para todo $T \in \mathcal{U}$ e $u, v \in H_{+}$,

$$
\langle u, v\rangle=\left\langle S^{+}(T) P_{T}^{+} T P_{T}^{+} S^{+}(T) u, v\right\rangle .
$$

Dado que a aplicação $T \mapsto A(T)$ para $T \in \mathcal{U}$ é contínua e $S^{+}(T)=\left(A(T)^{-1}\right)^{1 / 2}$, se segue dos Lemas 1.1.9 e 5.1.12 que $S^{+}$é contínua.

Por outro lado, tomemos a forma bilinear

$$
B_{-}(T)(u, v)=-\left\langle T Q_{T}^{-} u, Q_{T}^{-} v\right\rangle \quad \text { para } u, v \in H_{-} .
$$

Então, $B_{-}(T)$ é limitada e definida positiva. Como acima, podemos construir uma aplicação contínua

$$
\begin{aligned}
S^{-}: \mathcal{U} & \rightarrow G L\left(H_{-}\right) \\
T & \mapsto S^{-}(T)=S_{T}^{-}
\end{aligned}
$$

com a propriedade que, para todo $T \in \mathcal{U}$ e $u, v \in H_{-}$,

$$
-\langle u, v\rangle=\left\langle S^{-}(T) P_{T}^{-} T P_{T}^{-} S^{-}(T) u, v\right\rangle .
$$

Passo 3: A aplicação $S: \mathcal{U} \rightarrow G L(H)$ dada por

$$
S(T)=P_{T}^{+} S_{T}^{+} P_{\mathcal{J}}^{+}-P_{T}^{-} S_{T}^{-} P_{\mathcal{J}}^{-}
$$

é contínua e $S(T)^{*} T S(T)=\mathcal{J}$ para todo $T \in \mathcal{U}$. Dado que $P_{T}^{+} S_{T}^{+} P_{\mathcal{J}}^{+}$e $P_{T}^{-} S_{T}^{-} P_{\mathcal{J}}^{-}$ são contínuas, se segue que $S$ é contínua. Das igualdades (5.1.9) e (5.1.10) temos, respectivamente, que

$$
\left.S_{T}^{+} P_{T}^{+} T P_{T}^{+} S_{T}^{+}\right|_{H_{+}}=\left.I\right|_{H_{+}} \quad \text { e }\left.\quad S_{T}^{-} P_{T}^{-} T P_{T}^{-} S_{T}^{-}\right|_{H_{-}}=-\left.I\right|_{H_{-}} .
$$


Assim, como $P_{T}^{-} T P_{T}^{+}=0=P_{T}^{+} T P_{T}^{-}$e $S_{T}^{+}, S_{T}^{-}$e as projeções são auto-adjuntas, então

$$
\begin{aligned}
S(T)^{*} T S(T) & =\left(P_{T}^{+} S_{T}^{+} P_{\mathcal{J}}^{+}-P_{T}^{-} S_{T}^{-} P_{\mathcal{J}}^{-}\right)^{*} T\left(P_{T}^{+} S_{T}^{+} P_{\mathcal{J}}^{+}-P_{T}^{-} S_{T}^{-} P_{\mathcal{J}}^{-}\right) \\
& =\left(\left(P_{T}^{+} S_{T}^{+} P_{\mathcal{J}}^{+}\right)^{*}-\left(P_{T}^{-} S_{T}^{-} P_{\mathcal{J}}^{-}\right)^{*}\right) T\left(P_{T}^{+} S_{T}^{+} P_{\mathcal{J}}^{+}-P_{T}^{-} S_{T}^{-} P_{\mathcal{J}}^{-}\right) \\
& =\left(P_{\mathcal{J}}^{+*} S_{T}^{+*} P_{T}^{+*}-P_{\mathcal{J}}^{-*} S_{T}^{-*} P_{T}^{-*}\right) T\left(P_{T}^{+} S_{T}^{+} P_{\mathcal{J}}^{+}-P_{T}^{-} S_{T}^{-} P_{\mathcal{J}}^{-}\right) \\
& =\left(P_{\mathcal{J}}^{+} S_{T}^{+} P_{T}^{+}-P_{\mathcal{J}}^{-} S_{T}^{-} P_{T}^{-}\right) T\left(P_{T}^{+} S_{T}^{+} P_{\mathcal{J}}^{+}-P_{T}^{-} S_{T}^{-} P_{\mathcal{J}}^{-}\right) \\
& =P_{\mathcal{J}}^{+} S_{T}^{+} P_{T}^{+} T\left(P_{T}^{+} S_{T}^{+} P_{\mathcal{J}}^{+}-P_{T}^{-} S_{T}^{-} P_{\mathcal{J}}^{-}\right)-P_{\mathcal{J}}^{-} S_{T}^{-} P_{T}^{-} T\left(P_{T}^{+} S_{T}^{+} P_{\mathcal{J}}^{+}-P_{T}^{-} S_{T}^{-} P_{\mathcal{J}}^{-}\right) \\
& =P_{\mathcal{J}}^{+} S_{T}^{+} P_{T}^{+} T P_{T}^{+} S_{T}^{+} P_{\mathcal{J}}^{+}+P_{\mathcal{J}}^{-} S_{T}^{-} P_{T}^{-} T P_{T}^{-} S_{T}^{-} P_{\mathcal{J}}^{-} \\
& =P_{\mathcal{J}}^{+} I P_{\mathcal{J}}^{+}-P_{\mathcal{J}}^{-} I P_{\mathcal{J}}^{-} \\
& =\left(\left.I\right|_{H_{+}}\right) \oplus\left(-\left.I\right|_{H_{-}}\right) \\
& =\mathcal{J} .
\end{aligned}
$$

Daí, para todo $T \in \mathcal{U}, S(T)^{*} T S(T)=\mathcal{J}$.

Passo 4: A aplicação

$$
\begin{aligned}
\varrho: \mathcal{U} & \rightarrow \mathcal{G} \\
T & \mapsto\left(\left(S(T)^{-1}\right)^{*}, 0\right)
\end{aligned}
$$

é contínua e $\pi(\varrho(T))=\left.I\right|_{\mathcal{U}}$. De fato, é claro que $\varrho$ é contínua. Além disso, para $T \in \mathcal{U}$,

$$
\pi(\varrho(T))=\pi\left(\left(S(T)^{-1}\right)^{*}, 0\right)=\left(S(T)^{-1}\right)^{*} \mathcal{J} S(T)^{-1}=T .
$$

A prova é concluída.

Lema 5.1.14. A aplicação $\pi: \mathcal{G} \rightarrow \Phi_{S}^{i}(H)$ é sobrejetora.

Demonstração. Sejam $L \in \Phi_{S}^{i}(H)$ e $K$ a projeção ortogonal sobre Ker $L$. Da Observação 3.4.4 se segue que $T=L+K$ é um isomorfismo auto-adjunto. Além disso, o Lema 4.4.11 mostra que $T \in \Phi_{S}^{i}(H)$. Como consequência do Teorema 1.1.18, temos que existe um isomorfismo $A=A_{+} \oplus A_{-}$em $L(H)$ tal que $A_{+}: H_{+}(\mathcal{J}) \rightarrow H_{+}(T)$ e $A_{-}: H_{-}(\mathcal{J}) \rightarrow H_{-}(T)$ são isomorfismos. Sejam

$$
B_{+}(T)(u, v)=\left\langle T A_{+} u, A_{+} v\right\rangle \quad \text { para } u, v \in H_{+}(\mathcal{J})
$$

e

$$
B_{-}(T)(u, v)=-\left\langle T A_{-} u, A_{-} v\right\rangle \quad \text { para } u, v \in H_{-}(\mathcal{J}) .
$$

É fácil ver que $B_{+}(T)$ e $B_{-}(T)$ são formas bilineares definidas positivas. Procedendo analogamente à prova do Lema 5.1.13, com $A_{ \pm}$em vez de $Q_{T}^{ \pm}$, podemos mostrar que existe um elemento $S(T) \in G L(H)$ tal que $\left(S(T)^{-1}\right)^{*} \mathcal{J} S(T)^{-1}=T$. Daí,

$$
L=\left(S(T)^{-1}\right)^{*} \mathcal{J} S(T)^{-1}-K=\pi\left(\left(S(T)^{-1}\right)^{*},-K\right)
$$

Logo, $\pi$ é sobrejetora. 
Proposição 5.1.15. A aplicação $\pi: \mathcal{G} \rightarrow \Phi_{S}^{i}(H)$ é a projeção sobre a base de um fibrado localmente trivial com fibra $\pi^{-1}(\mathcal{J})$.

Demonstração. Pelo Lema 5.1.13 podemos escolher uma vizinhança $\mathcal{U}$ de $\mathcal{J}$ e uma aplicação $\varrho: \mathcal{U} \rightarrow \mathcal{G}$ tal que $\pi \varrho=\left.I\right|_{\mathcal{U}}$. Suponhamos que $L \in \mathcal{U}$ e $g \in \pi^{-1}(\mathcal{J})$. Então, se $\varrho(L)=(M, K)$,

$$
L=\pi \varrho(L)=M \mathcal{J} M^{*}+K
$$

Portanto, dado que $\pi(g)=\mathcal{J}$, de (5.1.4) temos

$$
\pi(\varrho(L) \cdot g)=M \pi(g) M^{*}+K=M \mathcal{J} M^{*}+K=L .
$$

Este fato prova que, se $L \in \mathcal{U}$ e $g \in \pi^{-1}(\mathcal{J})$, então $\varrho(L) \cdot g \in \pi^{-1}(\mathcal{U})$. Daí, podemos definir

$$
\begin{aligned}
\eta: \mathcal{U} \times \pi^{-1}(\mathcal{J}) & \rightarrow \pi^{-1}(\mathcal{U}) \\
(L, g) & \mapsto \varrho(L) \cdot g=\varsigma(\varrho(L), g) .
\end{aligned}
$$

A aplicação $\eta$ é contínua, pois $\varrho$ e $\varsigma$ são contínuas (veja-se (5.1.3)).

Observe que a aplicação

$$
\vartheta(g)=\left(\pi(g),[\varrho(\pi(g))]^{-1} \cdot g\right) \quad \text { para } g \in \pi^{-1}(\mathcal{U})
$$

é contínua e é a inversa de $\eta$. De fato, se $g \in \pi^{-1}(\mathcal{U})$ temos

$$
\eta \vartheta(g)=\eta\left(\pi(g),[\varrho(\pi(g))]^{-1} \cdot g\right)=\varrho(\pi(g)) \cdot[\varrho(\pi(g))]^{-1} \cdot g=g .
$$

Além disso, se $(L, g) \in \mathcal{U} \times \pi^{-1}(\mathcal{J})$, de (5.1.11) se segue

$$
\begin{aligned}
\vartheta \eta(L, g) & =\vartheta(\varrho(L) \cdot g)=\left(\pi(\varrho(L) \cdot g),[\varrho(\pi(\varrho(L) \cdot g))]^{-1} \cdot(\varrho(L) \cdot g)\right) \\
& =\left(L,[\varrho(L)]^{-1} \cdot(\varrho(L) \cdot g)\right)=(L, g) .
\end{aligned}
$$

Por outro lado, de (5.1.11) temos que $\pi \eta(L, g)=L$ para todo $L \in \mathcal{U}$ e $g \in \pi^{-1}(\mathcal{J})$. Assim, $\eta$ define uma trivialização sobre uma vizinhança de $\mathcal{J}$.

Usando a transitividade da ação, esta trivialização pode ser transportada a qualquer operador em $\Phi_{S}^{i}(H)$. De fato, fixemos $L_{0}$ em $\Phi_{S}^{i}(H)$. Da sobrejetividade de $\pi$ podemos escolher um elemento $g_{0}$ em $\mathcal{G}$ tal que $\pi\left(g_{0}\right)=\tau_{g_{0}}(\mathcal{J})=L_{0}$. Definamos $\mathcal{U}^{\prime}=\tau_{g_{0}}(\mathcal{U})$. Então, $\mathcal{U}^{\prime}$ é uma vizinhança de $L_{0}$ e $\tau_{g_{0}}(\cdot): \mathcal{U} \rightarrow \mathcal{U}^{\prime}$ é um homeomorfismo. Sejam $L^{\prime} \in \mathcal{U}^{\prime}$ e $g \in \pi^{-1}(\mathcal{J})$. Assim, $\pi(g)=\mathcal{J}$ e

$$
\tau_{g_{0}^{-1}}\left(L^{\prime}\right) \in \tau_{g_{0}^{-1}}\left(\mathcal{U}^{\prime}\right)=\tau_{g_{0}^{-1}}\left(\tau_{g_{0}}(\mathcal{U})\right)=\mathcal{U}
$$

isto é, $\tau_{g_{0}^{-1}}\left(L^{\prime}\right) \in \mathcal{U}$. Logo, o Lema 5.1.13 implica que $\pi \varrho\left(\tau_{g_{0}^{-1}}\left(L^{\prime}\right)\right)=\tau_{g_{0}^{-1}}\left(L^{\prime}\right)$. Se $\varrho\left(\tau_{g_{0}^{-1}}\left(L^{\prime}\right)\right)=\left(M^{\prime}, K^{\prime}\right)$, então

$$
\tau_{g_{0}^{-1}}\left(L^{\prime}\right)=\pi \varrho\left(\tau_{g_{0}^{-1}}\left(L^{\prime}\right)\right)=M^{\prime} \mathcal{J}\left(M^{\prime}\right)^{*}+K^{\prime} .
$$


Portanto, de (5.1.4) se segue

$$
\begin{aligned}
\pi\left(g_{0} \cdot \varrho\left(\tau_{g_{0}^{-1}}\left(L^{\prime}\right)\right) \cdot g\right) & =\pi \varsigma_{g_{0}}\left(\varrho\left(\tau_{g_{0}^{-1}}\left(L^{\prime}\right)\right) \cdot g\right)=\tau_{g_{0}} \pi\left(\varrho\left(\tau_{g_{0}^{-1}}\left(L^{\prime}\right)\right) \cdot g\right) \\
& =\tau_{g_{0}} \pi\left(\left(M^{\prime}, K^{\prime}\right) \cdot g\right)=\tau_{g_{0}}\left(M^{\prime} \pi(g)\left(M^{\prime}\right)^{*}+K^{\prime}\right) \\
& =\tau_{g_{0}}\left(M^{\prime} \mathcal{J}\left(M^{\prime}\right)^{*}+K^{\prime}\right)=\tau_{g_{0}}\left(\tau_{g_{0}^{-1}}\left(L^{\prime}\right)\right) \\
& =L^{\prime} .
\end{aligned}
$$

Daí, para $L^{\prime} \in \mathcal{U}^{\prime}$ e $g \in \pi^{-1}(\mathcal{J})$, temos

$$
\pi\left(g_{0} \cdot \varrho\left(\tau_{g_{0}^{-1}}\left(L^{\prime}\right)\right) \cdot g\right)=L^{\prime} \quad \text { e } \quad g_{0} \cdot \varrho\left(\tau_{g_{0}^{-1}}\left(L^{\prime}\right)\right) \cdot g \in \pi^{-1}\left(\mathcal{U}^{\prime}\right) .
$$

Tomemos a aplicação

$$
\begin{aligned}
\eta^{\prime}: \mathcal{U}^{\prime} \times \pi^{-1}(\mathcal{J}) & \rightarrow \pi^{-1}\left(\mathcal{U}^{\prime}\right) \\
\left(L^{\prime}, g\right) & \mapsto g_{0} \cdot \varrho\left(\tau_{g_{0}^{-1}}\left(L^{\prime}\right)\right) \cdot g .
\end{aligned}
$$

Provemos que

$$
\vartheta^{\prime}(g)=\left(\pi(g),\left[g_{0} \cdot \varrho\left(\tau_{g_{0}^{-1}}(\pi(g))\right)\right]^{-1} \cdot g\right) \quad \text { para } g \in \pi^{-1}\left(\mathcal{U}^{\prime}\right)
$$

é a inversa de $\eta$. Seja $g$ em $\pi^{-1}\left(\mathcal{U}^{\prime}\right)$. Assim,

$\eta^{\prime} \vartheta^{\prime}(g)=\eta^{\prime}\left(\pi(g),\left[g_{0} \cdot \varrho\left(\tau_{g_{0}^{-1}}(\pi(g))\right)\right]^{-1} \cdot g\right)=g_{0} \cdot \varrho\left(\tau_{g_{0}^{-1}}(\pi(g)) \cdot\left[g_{0} \cdot \varrho\left(\tau_{g_{0}^{-1}}(\pi(g))\right)\right]^{-1} \cdot g=g\right.$.

Agora, se $\left(L^{\prime}, g\right) \in \mathcal{U}^{\prime} \times \pi^{-1}(\mathcal{J})$, de (5.1.12) se segue

$$
\begin{aligned}
\vartheta^{\prime} \eta^{\prime}\left(L^{\prime}, g\right) & =\vartheta^{\prime}\left(g_{0} \cdot \varrho\left(\tau_{g_{0}^{-1}}\left(L^{\prime}\right)\right) \cdot g\right) \\
& =\left(\pi\left(g_{0} \cdot \varrho \tau_{g_{0}^{-1}} L^{\prime} \cdot g\right),\left[g_{0} \cdot \varrho\left(\tau_{g_{0}^{-1}}\left[\pi\left(g_{0} \cdot \varrho \tau_{g_{0}^{-1}} L^{\prime} \cdot g\right)\right]\right)\right]^{-1} \cdot g_{0} \cdot \varrho \tau_{g_{0}^{-1}} L^{\prime} \cdot g\right) \\
& =\left(L^{\prime},\left[g_{0} \cdot \varrho\left(\tau_{g_{0}^{-1}} L^{\prime}\right)\right]^{-1} \cdot g_{0} \cdot \varrho \tau_{g_{0}^{-1}} L^{\prime} \cdot g\right)=\left(L^{\prime}, g\right) .
\end{aligned}
$$

Portanto, $\vartheta^{\prime}$ é a inversa de $\eta^{\prime}$. Além disso, $\vartheta^{\prime}$ é contínua, pois é composição de aplicações contínuas.

Dado que

$$
\pi_{1} \vartheta^{\prime}(g)=\pi(g) \quad \text { para todo } g \in \pi^{-1}\left(\mathcal{U}^{\prime}\right),
$$

a aplicação $\vartheta^{\prime}$ define uma trivialização sobre uma vizinhança $\mathcal{U}^{\prime}$ do operador $L_{0}$. Em conclusão, $\pi: \mathcal{G} \rightarrow \Phi_{S}^{i}(H)$ é um fibrado localmente trivial com fibra $\pi^{-1}(\mathcal{J})$.

Teorema 5.1.16 (Existência da parametrix cogradiente). Sejam $\Lambda$ um espaço topológico paracompacto e contrátil e $L=\left\{L_{\lambda}\right\}_{\lambda \in \Lambda}$ uma familia de operadores de Fredholm fortemente indefinidos. Então, L possui uma parametrix cogradiente. 
Demonstração. Se segue da proposição anterior que a aplicação $\pi: \mathcal{G} \rightarrow \Phi_{S}^{i}(H)$ é um fibrado localmente trivial com fibra $\pi^{-1}(\mathcal{J})$ e base $\Phi_{S}^{i}(H)$. Seja $\Xi$ o subespaço do produto $\Lambda \times \mathcal{G}$ definido por

$$
\Xi=\left\{(\lambda, g) \in \Lambda \times \mathcal{G}: \mathrm{L}_{\lambda}=\pi(g)\right\} .
$$

Consideremos o pullback $\mathrm{L}^{\star}(\pi): \Xi \rightarrow \Lambda$ induzido por $\mathrm{L}$ e $\pi$, isto é,

$$
\mathrm{L}^{\star}(\pi)(\lambda, g)=\lambda \quad \text { para todo }(\lambda, g) \in \Xi .
$$

Do Lema 5.1.7 temos que $\mathrm{L}^{\star}(\pi)$ é um fibrado localmente trivial com fibra $\pi^{-1}(\mathcal{J})$ e base $\Lambda$. Dado que $\Lambda$ é paracompacto e contrátil, se segue da Proposição 5.1.8 que $L^{\star}(\pi)$ é um fibrado trivial, isto é, existe um homeomorfismo $\phi: \Xi \rightarrow \Lambda \times \pi^{-1}(\mathcal{J})$ tal que

$$
\mathbf{L}^{\star}(\pi)(\lambda, g)=\lambda=\pi_{1} \phi(\lambda, g) \quad \text { para todo }(\lambda, g) \in \Xi,
$$

onde $\pi_{1}: \Lambda \times \pi^{-1}(\mathcal{J}) \rightarrow \Lambda$ é a projeção sobre a primeira coordenada. Consequentemente, $\phi$ é da forma

$$
\phi(\lambda, g)=(\lambda, \psi(\lambda, g)) \quad \text { para }(\lambda, g) \in \Xi,
$$

onde $\psi: \Xi \rightarrow \pi^{-1}(\mathcal{J})$ é uma aplicação contínua. Fixemos $g_{0} \in \pi^{-1}(\mathcal{J})$ e tomemos

$$
\begin{aligned}
\phi_{g_{0}}^{-1}: \Lambda & \rightarrow \Xi \\
\lambda & \mapsto \phi^{-1}\left(\lambda, g_{0}\right) .
\end{aligned}
$$

A aplicação $\phi_{g_{0}}^{-1}$ é contínua, pois $\phi^{-1}$ é contínua. Agora, como $\phi_{g_{0}}^{-1}(\lambda) \in \Xi$, de (5.1.14) se segue que $\phi_{g_{0}}^{-1}(\lambda)$ é da forma

$$
\phi_{g_{0}}^{-1}(\lambda)=\left(\lambda, \mathrm{M}_{\lambda}, \mathrm{K}_{\lambda}\right),
$$

onde $\mathrm{M}_{\lambda} \in G L(H), \mathrm{K}_{\lambda} \in K_{S}(H)$ e $\pi\left(\mathrm{M}_{\lambda}, \mathrm{K}_{\lambda}\right)=\mathrm{L}_{\lambda}$, para todo $\lambda \in \Lambda$. É claro que $\mathrm{M}=\left\{\mathrm{M}_{\lambda}\right\}_{\lambda \in \Lambda}$ e $\mathrm{K}=\left\{\mathrm{K}_{\lambda}\right\}_{\lambda \in \Lambda}$ são famílias contínuas, pois $\phi_{g_{0}}^{-1}$ é contínua. Já que $\pi\left(\mathrm{M}_{\lambda}, \mathrm{K}_{\lambda}\right)=\mathrm{L}_{\lambda}$ para $\lambda \in \Lambda$, obtemos que

$$
\mathrm{L}_{\lambda}=\pi\left(\mathrm{M}_{\lambda}, \mathrm{K}_{\lambda}\right)=\mathrm{M}_{\lambda} \mathcal{J} \mathrm{M}_{\lambda}^{*}+\mathrm{K}_{\lambda},
$$

o que prova o teorema.

\subsection{Fluxo espectral de caminhos de operadores de Fredholm auto-adjuntos}

No começo desta seção apresentaremos a definição e as propriedades principais do fluxo espectral para caminhos de operadores fortemente indefinidos com extremos inversíveis. 
A noção do fluxo espectral se pode estender a caminhos fechados em $\Phi_{S}^{i}(H)$ cujos extremos não necessariamente são isomorfismos, como mostraremos mais adiante. No final da seção daremos a definição para qualquer caminho (fechados ou com extremos inversíveis) de operadores de Fredholm auto-adjuntos.

Denotemos por $J$ o intervalo $[a, b]$. Observe que $J$ é contrátil. De fato, a aplicação

$$
\begin{aligned}
h: J \times[0,1] & \rightarrow J \\
(x, \lambda) & \mapsto x(1-\lambda)+a \lambda
\end{aligned}
$$

é contínua e, além disso, para todo $x \in J, h(x, 0)=x$ e $h(x, 1)=a$. Por outro lado, dado que $J$ é compacto, então é paracompacto.

Seja $L=\left\{L_{\lambda}\right\}_{\lambda \in J}$ um caminho de operadores auto-adjuntos fortemente indefinidos. O Teorema 5.1.16 implica que, para cada simetria $\mathcal{J} \in \Phi_{S}^{i}(H)$, L é cogradiente a um caminho de perturbaçoes compactas auto-adjuntas de $\mathcal{J}$. Deste fato temos a seguinte definição.

Definição 5.2.1 (Fluxo espectral). Seja $L=\left\{L_{\lambda}\right\}_{\lambda \in J}$ um caminho de operadores de Fredholm auto-adjuntos, fortemente indefinidos e com extremos inversíveis. O fluxo espectral de $\mathrm{L}$ no intervalo $J$ é definido por

$$
\operatorname{sf}(\mathrm{L}, J)=\frac{1}{2}\left[\operatorname{sign}_{\mathcal{J}}\left(\mathcal{J}+\mathrm{K}_{b}\right)-\operatorname{sign}_{\mathcal{J}}\left(\mathcal{J}+\mathrm{K}_{a}\right)\right],
$$

onde $\mathcal{J} \in \Phi_{S}^{i}(H)$ é uma simetria e $\left\{\mathcal{J}+\mathrm{K}_{\lambda}\right\}_{\lambda \in J}$ é um caminho de perturbações compactas auto-adjuntas de $\mathcal{J}$ que é cogradiente a $\mathbf{L}$.

Provemos que a definição acima é independente das escolhas da simetria $\mathcal{J}$ e da parametrix. De fato, suponhamos que $\mathcal{J}$ e $\mathcal{J}^{\prime}$ sejam duas simetrias de $H$ e que $\left\{\mathrm{M}_{\lambda}\right\}_{\lambda \in J}$ e $\left\{M_{\lambda}^{\prime}\right\}_{\lambda \in J}$ sejam duas parametrix cogradientes de $\left\{L_{\lambda}\right\}_{\lambda \in J}$ relativas a $\mathcal{J}$ e $\mathcal{J}^{\prime}$, respectivamente. Assim, para $\lambda \in J$,

$$
\mathrm{M}_{\lambda}^{*} \mathrm{~L}_{\lambda} \mathrm{M}_{\lambda}=\mathcal{J}+\mathrm{K}_{\lambda} \quad \text { e } \quad\left(\mathrm{M}_{\lambda}^{\prime}\right)^{*} \mathrm{~L}_{\lambda} \mathrm{M}_{\lambda}^{\prime}=\mathcal{J}^{\prime}+\mathrm{K}_{\lambda}^{\prime},
$$

onde $\mathrm{K}_{\lambda}$ e $\mathrm{K}_{\lambda}^{\prime}$ são operadores compactos auto-adjuntos. Observe que os caminhos $\left\{\mathcal{J}+K_{\lambda}\right\}_{\lambda \in J}$ e $\left\{\mathcal{J}^{\prime}+K_{\lambda}^{\prime}\right\}_{\lambda \in J}$ são cogradientes. De fato, para $\lambda \in J$,

$$
\mathcal{J}^{\prime}+\mathrm{K}_{\lambda}^{\prime}=\left(\mathrm{M}_{\lambda}^{\prime}\right)^{*}\left(\mathrm{M}_{\lambda}^{*}\right)^{-1}\left(\mathcal{J}+\mathrm{K}_{\lambda}\right) \mathrm{M}_{\lambda}^{-1} \mathrm{M}_{\lambda}^{\prime}=\left(\mathrm{M}_{\lambda}^{-1} \mathrm{M}_{\lambda}^{\prime}\right)^{*}\left(\mathcal{J}+\mathrm{K}_{\lambda}\right) \mathrm{M}_{\lambda}^{-1} \mathrm{M}_{\lambda}^{\prime},
$$

Consequentemente,

$$
\mathcal{J}^{\prime}+\mathrm{K}_{\lambda}^{\prime}=\mathrm{N}_{\lambda}^{*}\left(\mathcal{J}+\mathrm{K}_{\lambda}\right) \mathrm{N}_{\lambda}
$$

onde $\mathrm{N}_{\lambda}=\mathrm{M}_{\lambda}^{-1} \mathrm{M}_{\lambda}^{\prime}$.

A Proposição 4.4.16 prova que

$$
\frac{1}{2}\left[\operatorname{sign}_{\mathcal{J}}\left(\mathcal{J}+\mathrm{K}_{b}\right)-\operatorname{sign}_{\mathcal{J}}\left(\mathcal{J}+\mathrm{K}_{a}\right)\right]=\mu_{\text {rel }}\left(\mathcal{J}+\mathrm{K}_{a}, \mathcal{J}+\mathrm{K}_{b}\right) .
$$


Da invariança do índice de Morse relativo pela ação cogradiente (Proposição 4.4.14), temos

$$
\mu_{\text {rel }}\left(\mathcal{J}+\mathrm{K}_{a}, \mathcal{J}+\mathrm{K}_{b}\right)=\mu_{\text {rel }}\left(\mathrm{N}_{a}^{*}\left(\mathcal{J}+\mathrm{K}_{a}\right) \mathrm{N}_{a}, \mathrm{~N}_{a}^{*}\left(\mathcal{J}+\mathrm{K}_{b}\right) \mathrm{N}_{a}\right) .
$$

Por outro lado, observe que, para cada $\lambda \in J, \mathrm{~N}_{\lambda}^{*} \mathcal{J} \mathrm{N}_{\lambda}$ é uma perturbação compacta de $\mathcal{J}^{\prime}$, pois $\mathrm{N}_{\lambda}^{*} \mathcal{J} \mathrm{N}_{\lambda}=\mathcal{J}^{\prime}+\mathrm{K}_{\lambda}^{\prime}-\mathrm{N}_{\lambda}^{*} \mathrm{~K}_{\lambda} \mathrm{N}_{\lambda}$. Assim, se $\lambda \in J$, então

$$
\begin{aligned}
\mathrm{N}_{a}^{*}\left(\mathcal{J}+\mathrm{K}_{a}\right) \mathrm{N}_{a}-\mathrm{N}_{\lambda}^{*}\left(\mathcal{J}+\mathrm{K}_{b}\right) \mathrm{N}_{\lambda} & =\mathcal{J}^{\prime}+\mathrm{K}_{a}^{\prime}-\mathrm{N}_{\lambda}^{*} \mathcal{J} \mathrm{N}_{\lambda}-\mathrm{N}_{\lambda}^{*} \mathrm{~K}_{b} \mathrm{~N}_{\lambda} \\
& =\mathcal{J}^{\prime}+\mathrm{K}_{a}^{\prime}-\left(\mathcal{J}^{\prime}+\mathrm{K}_{\lambda}^{\prime}-\mathrm{N}_{\lambda}^{*} \mathrm{~K}_{\lambda} \mathrm{N}_{\lambda}\right)-\mathrm{N}_{\lambda}^{*} \mathrm{~K}_{b} \mathrm{~N}_{\lambda} \\
& =\mathrm{K}_{a}^{\prime}-\mathrm{K}_{\lambda}^{\prime}+\mathrm{N}_{\lambda}^{*} \mathrm{~K}_{\lambda} \mathrm{N}_{\lambda}-\mathrm{N}_{\lambda}^{*} \mathrm{~K}_{b} \mathrm{~N}_{\lambda} .
\end{aligned}
$$

Logo,

$$
\mathrm{N}_{a}^{*}\left(\mathcal{J}+\mathrm{K}_{a}\right) \mathrm{N}_{a}-\mathrm{N}_{\lambda}^{*}\left(\mathcal{J}+\mathrm{K}_{b}\right) \mathrm{N}_{\lambda}
$$

é compacto para cada $\lambda \in J$. Este fato prova que

$$
\left\{\left(\mathrm{N}_{a}^{*}\left(\mathcal{J}+\mathrm{K}_{a}\right) \mathrm{N}_{a}, \mathrm{~N}_{\lambda}^{*}\left(\mathcal{J}+\mathrm{K}_{b}\right) \mathrm{N}_{\lambda}\right)\right\}_{\lambda \in J}
$$

é um caminho de pares de isomorfismos auto-adjuntos congruentes. Assim, pela continuidade do índice de Morse relativo (Proposição 4.4.12), temos

$$
\mu_{\text {rel }}\left(\mathrm{N}_{a}^{*}\left(\mathcal{J}+\mathrm{K}_{a}\right) \mathrm{N}_{a}, \mathrm{~N}_{a}^{*}\left(\mathcal{J}+\mathrm{K}_{b}\right) \mathrm{N}_{a}\right)=\mu_{\text {rel }}\left(\mathrm{N}_{a}^{*}\left(\mathcal{J}+\mathrm{K}_{a}\right) \mathrm{N}_{a}, \mathrm{~N}_{b}^{*}\left(\mathcal{J}+\mathrm{K}_{b}\right) \mathrm{N}_{b}\right) .
$$

Da Proposição 4.4.16 se segue

$$
\mu_{\text {rel }}\left(\mathcal{J}^{\prime}+\mathrm{K}_{a}^{\prime}, \mathcal{J}^{\prime}+\mathrm{K}_{b}^{\prime}\right)=\frac{1}{2}\left[\operatorname{sign}_{\mathcal{J}^{\prime}}\left(\mathcal{J}^{\prime}+\mathrm{K}_{b}^{\prime}\right)-\operatorname{sign}_{\mathcal{J}^{\prime}}\left(\mathcal{J}^{\prime}+\mathrm{K}_{a}^{\prime}\right)\right] .
$$

Dado que $\mathrm{N}_{a}^{*}\left(\mathcal{J}+\mathrm{K}_{a}\right) \mathrm{N}_{a}=\mathcal{J}^{\prime}+\mathrm{K}_{a}^{\prime}$ e $\mathrm{N}_{b}^{*}\left(\mathcal{J}+\mathrm{K}_{b}\right) \mathrm{N}_{b}=\mathcal{J}^{\prime}+\mathrm{K}_{b}^{\prime}$, das igualdades (5.2.2)-(5.2.5) concluímos que

$$
\frac{1}{2}\left[\operatorname{sign}_{\mathcal{J}}\left(\mathcal{J}+\mathrm{K}_{b}\right)-\operatorname{sign}_{\mathcal{J}}\left(\mathcal{J}+\mathrm{K}_{a}\right)\right]=\frac{1}{2}\left[\operatorname{sign}_{\mathcal{J}^{\prime}}\left(\mathcal{J}^{\prime}+\mathrm{K}_{b}^{\prime}\right)-\operatorname{sign}_{\mathcal{J}^{\prime}}\left(\mathcal{J}^{\prime}+\mathrm{K}_{a}^{\prime}\right)\right]
$$

Consequentemente, a definição do fluxo espectral é independente da escolha da simetria e da correspondente escolha da parametrix cogradiente.

Observação 5.2.2. Observe que, de (5.2.1), a relação "ser cogradiente a" é transitiva. É fácil ver que esta relação é simétrica e reflexiva, portanto ela define uma relação de equivalência.

Definição 5.2.3. Um caminho de operadores com extremos inversíveis será chamado de admissivel.

Vejamos agora algumas propriedades do fluxo espectral. No resto da seção $L$ denotará um caminho $\left\{\mathrm{L}_{\lambda}\right\}_{\lambda \in J}$ em $\Phi_{S}^{i}(H)$ e $J$ o intervalo $[a, b]$. 
Proposição 5.2.4 (Normalização). Se L é um caminho de isomorfismos, então

$$
\operatorname{sf}(L, J)=0 .
$$

Demonstração. Seja $\left\{\mathcal{J}+\mathrm{K}_{\lambda}\right\}_{\lambda \in J}$ um caminho cogradiente a L. É claro que, para todo $\lambda \in J, \mathcal{J}+\mathrm{K}_{\lambda}$ é um isomorfismo, pois é cogradiente ao isomorfismo $\mathrm{L}_{\lambda}$. Da Proposição 4.4.16 temos

$$
\operatorname{sf}(\mathrm{L}, J)=\frac{1}{2}\left[\operatorname{sign}_{\mathcal{J}}\left(\mathcal{J}+\mathrm{K}_{b}\right)-\operatorname{sign}_{\mathcal{J}}\left(\mathcal{J}+\mathrm{K}_{a}\right)\right]=\mu_{\text {rel }}\left(\mathcal{J}+\mathrm{K}_{a}, \mathcal{J}+\mathrm{K}_{b}\right) .
$$

Como $\left\{\mathcal{J}+\mathrm{K}_{\lambda}, \mathcal{J}+\mathrm{K}_{b}\right\}_{\lambda \in J}$ é um caminho de pares de isomorfismos auto-adjuntos congruentes, a Proposição 4.4.12 implica que $\mu_{\text {rel }}\left(\mathcal{J}+\mathrm{K}_{\lambda}, \mathcal{J}+\mathrm{K}_{b}\right)$ é constante para $\lambda \in J$. Isto é, para cada $\lambda \in J$,

$$
\mu_{\text {rel }}\left(\mathcal{J}+\mathrm{K}_{a}, \mathcal{J}+\mathrm{K}_{b}\right)=\mu_{\text {rel }}\left(\mathcal{J}+\mathrm{K}_{\lambda}, \mathcal{J}+\mathrm{K}_{b}\right)=\mu_{\text {rel }}\left(\mathcal{J}+\mathrm{K}_{b}, \mathcal{J}+\mathrm{K}_{b}\right)=0 .
$$

Portanto, $\operatorname{sf}(\mathrm{L}, J)=0$.

Em seguida apresentaremos uma das mais importantes propriedades do fluxo espectral, isto é, a sua invariâcia pela ação cogradiente.

Proposição 5.2.5 (Invariância pela ação cogradiente). Seja $L$ um caminho admissivel. Então, para qualquer caminho $M=\left\{M_{\lambda}\right\}_{\lambda \in J}$ de operadores em $G L(H)$,

$$
\operatorname{sf}(L, J)=\operatorname{sf}\left(M^{*} L M, J\right),
$$

onde $M^{*} L M=\left\{M_{\lambda}^{*} L_{\lambda} M_{\lambda}\right\}_{\lambda \in J}$.

Demonstração. Seja $\left\{\mathcal{J}+\mathrm{K}_{\lambda}\right\}_{\lambda \in J}$ um caminho cogradiente a L. Observe que o caminho $\left\{\mathcal{J}+\mathrm{K}_{\lambda}\right\}_{\lambda \in J}$ também é cogradiente a $\mathrm{M}^{*} \mathrm{LM}$. Portanto,

$$
\operatorname{sf}(\mathrm{L}, J)=\frac{1}{2}\left[\operatorname{sign}_{\mathcal{J}}\left(\mathcal{J}+\mathrm{K}_{b}\right)-\operatorname{sign}_{\mathcal{J}}\left(\mathcal{J}+\mathrm{K}_{a}\right)\right]=\operatorname{sf}\left(\mathrm{M}^{*} \mathrm{LM}, J\right)
$$

pela definição do fluxo espectral.

Proposição 5.2.6 (Fluxo espectral para perturbações compactas de um operador fixado). Seja $L$ um caminho admissivel tal que $L_{\lambda}-L_{a}$ é compacto para todo $\lambda \in J$. Então,

$$
\operatorname{sf}(L, J)=\mu_{r e l}\left(L_{a}, L_{b}\right) .
$$

Demonstração. Por hipótese, para cada $\lambda \in J$, podemos escrever $\mathrm{L}_{\lambda}=\mathrm{L}_{a}+\mathrm{K}_{\lambda}$, onde $\mathrm{K}_{\lambda}$ é compacto e auto-adjunto. Escolhamos um operador $\mathrm{M} \in G L(H)$ tal que $\mathrm{M}^{*} \mathrm{~L}_{a} \mathrm{M}=$ $\mathcal{J}+\mathrm{K}$, onde $\mathrm{K} \in K_{S}(H)$ (isto é possível aplicando o Teorema 5.1.16 ao caminho constante $\left.\left\{\mathrm{L}_{a}\right\}_{\lambda \in J}\right)$. Logo,

$$
\mathrm{M}^{*} \mathrm{~L}_{\lambda} \mathrm{M}=\mathcal{J}+\mathrm{C}_{\lambda}
$$


onde $\mathrm{C}_{\lambda}=\mathrm{K}+\mathrm{M}^{*} \mathrm{~K}_{\lambda} \mathrm{M}$ é compacto para todo $\lambda \in J$. Consequentemente, o caminho constante $\mathrm{M}=\{\mathrm{M}\}_{\lambda \in J}$ é uma parametrix cogradiente para L. Este fato prova que $\left\{\mathcal{J}+\mathrm{C}_{\lambda}\right\}_{\lambda \in J}$ é cogradiente a L. Assim, pela definição de fluxo espectral,

$$
\operatorname{sf}(\mathrm{L}, J)=\mu_{r e l}\left(\mathcal{J}+\mathrm{C}_{a}, \mathcal{J}+\mathrm{C}_{b}\right)=\mu_{\text {rel }}\left(\mathrm{M}^{*} \mathrm{~L}_{a} \mathrm{M}, \mathrm{M}^{*} \mathrm{~L}_{b} \mathrm{M}\right) .
$$

Dado que $\mu_{r e l}\left(\mathrm{M}^{*} \mathrm{~L}_{a} \mathrm{M}, \mathrm{M}^{*} \mathrm{~L}_{b} \mathrm{M}\right)=\mu_{\text {rel }}\left(\mathrm{L}_{a}, \mathrm{~L}_{b}\right)$, então $\operatorname{sf}(\mathrm{L}, J)=\mu_{\text {rel }}\left(\mathrm{L}_{a}, \mathrm{~L}_{b}\right)$.

Proposição 5.2.7 (Aditividade). Se o caminho L é admissivel em $[a, c]$ e em $[c, b]$, então

$$
\operatorname{sf}(L,[a, b])=\operatorname{sf}(L,[a, c])+\operatorname{sf}(L,[c, b]) .
$$

Demonstração. De fato, seja $\left\{\mathcal{J}+\mathrm{K}_{\lambda}\right\}_{\lambda \in J}$ um caminho cogradiente a L. Então,

$$
\begin{aligned}
\operatorname{sf}(\mathrm{L},[a, c])+ & \operatorname{sf}(\mathrm{L},[c, b]) \\
& =\frac{1}{2}\left[\operatorname{sign}_{\mathcal{J}}\left(\mathcal{J}+\mathrm{K}_{c}\right)-\operatorname{sign}_{\mathcal{J}}\left(\mathcal{J}+\mathrm{K}_{a}\right)+\operatorname{sign}_{\mathcal{J}}\left(\mathcal{J}+\mathrm{K}_{b}\right)-\operatorname{sign}_{\mathcal{J}}\left(\mathcal{J}+\mathrm{K}_{c}\right)\right] \\
& =\frac{1}{2}\left[\operatorname{sign}_{\mathcal{J}}\left(\mathcal{J}+\mathrm{K}_{b}\right)-\operatorname{sign}_{\mathcal{J}}\left(\mathcal{J}+\mathrm{K}_{a}\right)\right] \\
& =\operatorname{sf}(\mathrm{L},[a, b]) .
\end{aligned}
$$

Este fato prova a proposição.

Para a seguinte proposição suporemos que $H_{1}$ e $H_{2}$ sejam espaços de Hilbert reais, separáveis e de dimensão infinita. Além disso, consideraremos dois caminhos $\mathrm{L}_{1}=$ $\left\{\mathrm{L}_{1, \lambda}\right\}_{\lambda \in J}$ e $\mathrm{L}_{2}=\left\{\mathrm{L}_{2, \lambda}\right\}_{\lambda \in J}$ em $\Phi_{S}^{i}\left(H_{1}\right)$ e $\Phi_{S}^{i}\left(H_{2}\right)$, respectivamente, e denotaremos por $\left(\mathrm{L}_{1}, \mathrm{~L}_{2}\right)=\left\{\left(\mathrm{L}_{1, \lambda}, \mathrm{L}_{2, \lambda}\right)\right\}_{\lambda \in J} \subseteq H_{1} \times H_{2}$ o produto direto destes caminhos.

Proposição 5.2.8 (Fluxo espectral de um produto direto de operadores). Se $L_{1}$ e $L_{2}$ são caminhos admissiveis de operadores de Fredholm auto-adjuntos fortemente indefinidos em $\mathrm{H}_{1}$ e $\mathrm{H}_{2}$, respectivamente, então

$$
\operatorname{sf}\left(\left(L_{1}, L_{2}\right), J\right)=\operatorname{sf}\left(L_{1}, J\right)+\operatorname{sf}\left(L_{2}, J\right) .
$$

Demonstração. Sejam $\left\{\mathcal{J}_{1}+\mathrm{K}_{1 \lambda}\right\}_{\lambda \in J}$ e $\left\{\mathcal{J}_{2}+\mathrm{K}_{2 \lambda}\right\}_{\lambda \in J}$ caminhos cogradientes a $\mathrm{L}_{1}$ e $\mathrm{L}_{2}$, respectivamente. Assim, existem dois caminhos $\mathrm{M}_{1}=\left\{\mathrm{M}_{1 \lambda}\right\}_{\lambda \in J}$ e $\mathrm{M}_{2}=\left\{\mathrm{M}_{2 \lambda}\right\}_{\lambda \in J}$ em $G L\left(H_{1}\right)$ e $G L\left(H_{2}\right)$, respectivamente, tais que, para cada $\lambda \in J$,

$$
\mathrm{M}_{1 \lambda}{ }^{*} \mathrm{~L}_{1 \lambda} \mathrm{M}_{1 \lambda}=\mathcal{J}_{1}+\mathrm{K}_{1 \lambda} \quad \text { e } \quad \mathrm{M}_{2 \lambda}{ }^{*} \mathrm{~L}_{2 \lambda} \mathrm{M}_{2 \lambda}=\mathcal{J}_{2}+\mathrm{K}_{2 \lambda} .
$$

De (3.2.1) temos a relação seguinte para caminhos de operadores em $H_{1}$ e $H_{2}$ :

$$
\begin{aligned}
\left(\mathrm{M}_{1 \lambda}, \mathrm{M}_{2 \lambda}\right)^{*}\left(\mathrm{~L}_{1 \lambda}, \mathrm{L}_{2 \lambda}\right)\left(\mathrm{M}_{1 \lambda}, \mathrm{M}_{2 \lambda}\right) & =\left(\mathrm{M}_{1 \lambda}{ }^{*}, \mathrm{M}_{2 \lambda}{ }^{*}\right)\left(\mathrm{L}_{1 \lambda}, \mathrm{L}_{2 \lambda}\right)\left(\mathrm{M}_{1 \lambda}, \mathrm{M}_{2 \lambda}\right) \\
& =\left(\mathrm{M}_{1 \lambda}{ }^{*} \mathrm{~L}_{1 \lambda} \mathrm{M}_{1 \lambda}, \mathrm{M}_{2 \lambda}{ }^{*} \mathrm{~L}_{2 \lambda} \mathrm{M}_{2 \lambda}\right) \\
& =\left(\mathcal{J}_{1}+\mathrm{K}_{1 \lambda}, \mathcal{J}_{2}+\mathrm{K}_{2 \lambda}\right) \\
& =\left(\mathcal{J}_{1}, \mathcal{J}_{2}\right)+\left(\mathrm{K}_{1 \lambda}, \mathrm{K}_{2 \lambda}\right) .
\end{aligned}
$$


Este fato prova que $\left\{\left(\mathcal{J}_{1}, \mathcal{J}_{2}\right)+\left(\mathrm{K}_{1 \lambda}, \mathrm{K}_{2 \lambda}\right)\right\}_{\lambda \in J}$ é cogradiente ao caminho de operadores $\left\{\left(\mathrm{L}_{1 \lambda}, \mathrm{L}_{2 \lambda}\right)\right\}_{\lambda \in J}$.

Em (4.2.11) provamos que $\left(\mathcal{J}_{1}, \mathcal{J}_{2}\right)$ é uma simetria. De $(3.2 .1)$ temos que $\left(\mathrm{K}_{1 \lambda}, \mathrm{K}_{2 \lambda}\right)$ é auto-adjunto. Assim, $\left(\mathrm{K}_{1 \lambda}, \mathrm{K}_{2 \lambda}\right)$ é compacto e auto-adjunto. Consequentemente, da definição do fluxo espectral e da Proposição 4.2.11, temos

$$
\begin{aligned}
\operatorname{sf} & \left(\left(\mathrm{L}_{1}, \mathrm{~L}_{2}\right), J\right) \\
& =\frac{1}{2}\left[\operatorname{sign}_{\left(\mathcal{J}_{1}, \mathcal{J}_{2}\right)}\left(\left(\mathcal{J}_{1}, \mathcal{J}_{2}\right)+\left(\mathrm{K}_{1 b}, \mathrm{~K}_{2 b}\right)\right)-\operatorname{sign}_{\left(\mathcal{J}_{1}, \mathcal{J}_{2}\right)}\left(\left(\mathcal{J}_{1}, \mathcal{J}_{2}\right)+\left(\mathrm{K}_{1 a}, \mathrm{~K}_{2 a}\right)\right)\right] \\
& =\frac{1}{2}\left[\operatorname{sign}_{\mathcal{J}_{1}}\left(\mathcal{J}_{1}+\mathrm{K}_{1 b}\right)+\operatorname{sign}_{\mathcal{J}_{2}}\left(\mathcal{J}_{2}+\mathrm{K}_{2 b}\right)-\left(\operatorname{sign}_{\mathcal{J}_{1}}\left(\mathcal{J}_{1}+\mathrm{K}_{1 a}\right)+\operatorname{sign}_{\mathcal{J}_{2}}\left(\mathcal{J}_{2}+\mathrm{K}_{2 a}\right)\right)\right] \\
& =\frac{1}{2}\left[\operatorname{sign}_{\mathcal{J}_{1}}\left(\mathcal{J}_{1}+\mathrm{K}_{1 b}\right)-\operatorname{sign}_{\mathcal{J}_{1}}\left(\mathcal{J}_{1}+\mathrm{K}_{1 a}\right)+\operatorname{sign}_{\mathcal{J}_{2}}\left(\mathcal{J}_{2}+\mathrm{K}_{2 b}\right)-\operatorname{sign}_{\mathcal{J}_{2}}\left(\mathcal{J}_{2}+\mathrm{K}_{2 a}\right)\right] \\
& =\operatorname{sf}\left(\mathrm{L}_{1}, J\right)+\operatorname{sf}\left(\mathrm{L}_{2}, J\right) .
\end{aligned}
$$

Isto é, $\operatorname{sf}\left(\left(\mathrm{L}_{1}, \mathrm{~L}_{2}\right), J\right)=\operatorname{sf}\left(\mathrm{L}_{1}, J\right)+\operatorname{sf}\left(\mathrm{L}_{2}, J\right)$.

Uma propriedade importante do fluxo espectral é a invariância homotópica. Apresentaremos aqui esta propriedade em várias versões. Primeiramente, observamos que $[0,1] \times J$ é contrátil. De fato, a aplicação

$$
h:[0,1] \times J \times[0,1] \rightarrow[0,1] \times J \quad(x, y, \lambda) \mapsto(x(1-\lambda), y(1-\lambda)+a \lambda)
$$

é contínua e, além disso, para todo $(x, y) \in[0,1] \times J$, temos

$$
h(x, y, 0)=(x, y) \quad \text { e } \quad h(x, y, 1)=(0, a) .
$$

Teorema 5.2.9 (Invariância homotópica). Seja

$$
L=\left\{L_{(s, \lambda)}\right\}_{(s, \lambda) \in[0,1] \times J}
$$

uma família de operadores em $\Phi_{S}^{i}(H)$ tal que, para cada $s \in[0,1]$, o caminho $L_{(s, \cdot)}=$ $\left\{L_{(s, \lambda)}\right\}_{\lambda \in J}$ é admissível. Então, $\operatorname{sf}\left(L_{(s, \cdot)}, J\right)$ é independente de s.

Demonstração. Dado que $[0,1] \times J$ é compacto e contrátil, o Teorema 5.1.16 implica que podemos escolher uma parametrix $\mathrm{M}=\left\{\mathrm{M}_{(s, \lambda)}\right\}_{(s, \lambda) \in[0,1] \times J}$ relativa a $\mathcal{J}$ para $\mathrm{L}$. Logo,

$$
\mathrm{M}_{(s, \lambda)}^{*} \mathrm{~L}_{(s, \lambda)} \mathrm{M}_{(s, \lambda)}=\mathcal{J}+\mathrm{K}_{(s, \lambda)} \text {, onde } \mathrm{K}_{(s, \lambda)} \in K_{S}(H) \text { para }(s, \lambda) \in[0,1] \times J .
$$

Dado $s \in[0,1]$, tomemos $\mathrm{M}_{(s, \cdot)}=\left\{\mathrm{M}_{(s, \lambda)}\right\}_{\lambda \in J}$. Da definição do fluxo espectral e da Proposição 4.4.16 temos que, para cada $s \in[0,1]$,

$$
\operatorname{sf}\left(\mathrm{L}_{(s, \cdot)}, J\right)=\operatorname{sf}\left(\mathrm{M}_{(s, \cdot)}^{*} \mathrm{~L}_{(s, \cdot)} \mathrm{M}_{(s, \cdot)}, J\right)=\mu_{r e l}\left(\mathrm{M}_{(s, a)}^{*} \mathrm{~L}_{(s, a)} \mathrm{M}_{(s, a)}, \mathrm{M}_{(s, b)}^{*} \mathrm{~L}_{(s, b)} \mathrm{M}_{(s, b)}\right)
$$


Já que, para cada $s \in[0,1], \mathrm{M}_{(s, a)}^{*} \mathrm{~L}_{(s, a)} \mathrm{M}_{(s, a)}$ e $\mathrm{M}_{(s, b)}^{*} \mathrm{~L}_{(s, b)} \mathrm{M}_{(s, b)}$ são isomorfismos auto-adjuntos congruentes, a Propoposição 4.4 .12 mostra que

$$
\mu_{r e l}\left(\mathrm{M}_{(s, a)}^{*} \mathrm{~L}_{(s, a)} \mathrm{M}_{(s, a)}, \mathrm{M}_{(s, b)}^{*} \mathrm{~L}_{(s, b)} \mathrm{M}_{(s, b)}\right)
$$

é constante em $[0,1]$. Este fato prova que $\operatorname{sf}\left(\mathrm{L}_{(s, \cdot)}, J\right)$ é independente de $s$.

A seguinte propriedade é uma outra versão da invariância homotópica do fluxo espectral, neste caso para caminhos cujos extremos são cogradientes.

Teorema 5.2.10 (Invariância homotópica de caminhos com extremos cogradientes). Seja $L=\left\{L_{(s, \lambda)}\right\}_{(s, \lambda) \in[0,1] \times J}$ uma família de operadores em $\Phi_{S}^{i}(H)$ tal que os caminhos $L_{(0, \cdot)}=\left\{L_{(0, \lambda)}\right\}_{\lambda \in J}$ e $L_{(1, \cdot)}=\left\{L_{(1, \lambda)}\right\}_{\lambda \in J}$ são admissiveis e os caminhos $L_{(\cdot, a)}=$ $\left\{L_{(s, a)}\right\}_{s \in[1,0]}$ e $L_{(\cdot, b)}=\left\{L_{(s, b)}\right\}_{s \in[1,0]}$ são cogradientes. Então,

$$
\operatorname{sf}\left(L_{(0, \cdot)}, J\right)=\operatorname{sf}\left(L_{(1, \cdot)}, J\right) .
$$

Demonstração. Escolhamos uma parametrix $\mathrm{M}=\left\{\mathrm{M}_{(s, \lambda)}\right\}_{(s, \lambda) \in[0,1] \times J}$ relativa a $\mathcal{J}$ para L. Sejam $\mathrm{M}_{(\cdot, a)}=\left\{\mathrm{M}_{(s, a)}\right\}_{s \in[1,0]}$ e $\mathrm{M}_{(\cdot, b)}=\left\{\mathrm{M}_{(s, b)}\right\}_{s \in[1,0]}$. Dado que, por hipótese, os caminhos $\mathrm{L}_{(\cdot, a)}$ e $\mathrm{L}_{(\cdot, b)}$ são cogradientes e admissíveis, temos que

$$
\left\{\mathrm{M}_{(s, a)}^{*} \mathrm{~L}_{(s, a)} \mathrm{M}_{(s, a)}=\mathcal{J}+\mathrm{K}_{(s, a)}\right\}_{s \in[0,1]} \quad \text { e } \quad\left\{\mathrm{M}_{(s, b)}^{*} \mathrm{~L}_{(s, b)} \mathrm{M}_{(s, b)}=\mathcal{J}+\mathrm{K}_{(s, b)}\right\}_{s \in[0,1]}
$$

são caminhos cogradientes admissíveis. Assim, a invariância do fluxo espectral pela ação cogradiente implica que

$$
\operatorname{sf}\left(\mathrm{M}_{(\cdot, a)}^{*} \mathrm{~L}_{(\cdot, a)} \mathrm{M}_{(\cdot, a)},[0,1]\right)=\operatorname{sf}\left(\mathrm{M}_{(\cdot, b)}^{*} \mathrm{~L}_{(\cdot, b)} \mathrm{M}_{(\cdot, b)},[0,1]\right) .
$$

Pela definição do fluxo espectral, esta igualdade pode ser expressada como

$$
\operatorname{sign}_{\mathcal{J}}\left(\mathcal{J}+\mathrm{K}_{(1, a)}\right)-\operatorname{sign}_{\mathcal{J}}\left(\mathcal{J}+\mathrm{K}_{(0, a)}\right)=\operatorname{sign}_{\mathcal{J}}\left(\mathcal{J}+\mathrm{K}_{(1, b)}\right)-\operatorname{sign}_{\mathcal{J}}\left(\mathcal{J}+\mathrm{K}_{(0, b)}\right) .
$$

Assim,

$$
\operatorname{sign}_{\mathcal{J}}\left(\mathcal{J}+\mathrm{K}_{(0, b)}\right)-\operatorname{sign}_{\mathcal{J}}\left(\mathcal{J}+\mathrm{K}_{(0, a)}\right)=\operatorname{sign}_{\mathcal{J}}\left(\mathcal{J}+\mathrm{K}_{(1, b)}\right)-\operatorname{sign}_{\mathcal{J}}\left(\mathcal{J}+\mathrm{K}_{(1, a)}\right),
$$

isto é,

$$
\operatorname{sf}\left(\mathcal{J}+\mathrm{K}_{(0, \cdot)}, J\right)=\operatorname{sf}\left(\mathcal{J}+\mathrm{K}_{(1, \cdot)}, J\right) .
$$

Portanto, $\operatorname{sf}\left(\mathrm{L}_{(0, \cdot)}, J\right)=\operatorname{sf}\left(\mathrm{L}_{(1, \cdot)}, J\right)$.

O teorema acima nós leva a estender a definição do fluxo espectral a caminhos fechados $\left(\mathrm{L}_{a}=\mathrm{L}_{b}\right)$ sem qualquer hipótese sobre inversibilidade dos extremos, como veremos na próxima definição. 
Definição 5.2.11 (Fluxo espectral para caminhos fechados). Sejam $\mathrm{L}$ um caminho fechado em $\Phi_{S}^{i}(H)$ e $K$ um operador compacto auto-adjunto tal que $\mathrm{L}_{a}+K=\mathrm{L}_{b}+K$ é inversível. O fluxo espectral de $L$ é dado por

$$
\operatorname{sf}(\mathrm{L}, J)=\operatorname{sf}(\mathrm{L}+K, J),
$$

onde $\mathrm{L}+K=\left\{\mathrm{L}_{\lambda}+K\right\}_{\lambda \in J}$.

Como vimos na Observação 3.4.4, para qualquer operador de Fredholm auto-adjunto $L$, podemos escolher um operador compacto auto-adjunto $K$ tal que $L+K$ é um isomorfismo auto-adjunto. Provemos que a definição acima é independente da escolha do operador $K$. De fato, sejam $\mathrm{L}$ um caminho fechado em $\Phi_{S}^{i}(H)$ e $K_{1}$ e $K_{2}$ dois operadores compactos auto-adjuntos tais que $\mathrm{L}_{a}+K_{1}=\mathrm{L}_{b}+K_{1}$ e $\mathrm{L}_{a}+K_{2}=\mathrm{L}_{b}+K_{2}$ sejam inversíveis. Tomemos $\mathrm{h}:[0,1] \times J \rightarrow \Phi_{S}^{i}(H)$ definido por

$$
\mathrm{h}(s, \lambda)=\mathrm{L}_{\lambda}+s K_{1}+(1-s) K_{2} \text { para }(s, \lambda) \in[0,1] \times J .
$$

Assim, os caminhos

$$
\mathrm{h}_{(0, \cdot)}=\left\{\mathrm{L}_{\lambda}+K_{2}\right\}_{\lambda \in J} \quad \text { e } \quad \mathrm{h}_{(1, \cdot)}=\left\{\mathrm{L}_{\lambda}+K_{1}\right\}_{\lambda \in J}
$$

são admissíveis. Observe que, como $\mathrm{L}_{a}+K_{2}=\mathrm{L}_{b}+K_{2}$, para todo $s \in[0,1]$,

$$
\mathrm{h}(s, a)=\mathrm{L}_{a}+s K_{1}+(1-s) K_{2}=\mathrm{L}_{b}+s K_{1}+(1-s) K_{2}=\mathrm{h}(s, b),
$$

isto é, $\mathrm{h}(s, a)=\mathrm{h}(s, b)$ para todo $s \in[0,1]$. Portanto, o teorema anterior implica que

$$
\operatorname{sf}\left(\mathrm{h}_{(0, \cdot)}, J\right)=\operatorname{sf}\left(\mathrm{h}_{(1, \cdot)}, J\right) .
$$

Logo,

$$
\operatorname{sf}\left(\mathrm{L}+K_{2}, J\right)=\operatorname{sf}\left(\mathrm{L}+K_{1}, J\right),
$$

o que prova que a fórmula (5.2.7) é independente da escolha do operador $K$.

O fluxo espectral de um caminho fechado tem a propriedade de invariância homotópica por homotopias de caminhos fechados. Este fato é provado na seguinte proposição.

Proposição 5.2.12 (Propriedade de homotopia livre para caminhos fechados). Seja $L=\left\{L_{(s, \lambda)}\right\}_{(s, \lambda) \in[0,1] \times J}$ uma familia de operadores em $\Phi_{S}^{i}(H)$ com a propriedade que $L_{(s, a)}=L_{(s, b)}$ para todo $s \in[0,1]$. Então,

$$
\operatorname{sf}\left(L_{(0, \cdot)}, J\right)=\operatorname{sf}\left(L_{(1, \cdot)}, J\right) .
$$


Demonstração. Observe que podemos escolher um operador compacto auto-adjunto $K^{\prime}$, tal que $\mathrm{L}_{(0, a)}+K^{\prime}$ e $\mathrm{L}_{(1, a)}+K^{\prime}$ sejam inversíveis. De fato, dado que a dimensão do núcleo de $\mathrm{L}_{(0, a)}$ é finita, a projeção ortogonal $P$ sobre $\operatorname{Ker} \mathrm{L}_{(0, a)}$ é compacta. Além disso,

$$
\mathrm{L}_{(0, a)}+P \quad \text { é inversível. }
$$

Agora, $\mathrm{L}_{(1, a)}+P$ é um operador de Fredholm auto-adjunto (é a soma de um operador de Fredholm e um operador compacto), portanto seu núcleo tem dimensão finita. Denotemos por $Q$ a projeção ortogonal sobre $\operatorname{Ker}\left(\mathrm{L}_{(1, a)}+P\right)$.

Vejamos que, para $\varepsilon>0$ suficientemente pequeno, o operador compacto $K^{\prime}=$ $P+\varepsilon Q$ tem a propriedade desejada. De fato, como $\mathrm{L}_{(0, a)}+P$ é inversível, então, para $\varepsilon$ suficientemente pequeno, temos que

$$
\mathrm{L}_{(0, a)}+K^{\prime}=\mathrm{L}_{(0, a)}+P+\varepsilon Q \text { é inversível. }
$$

Por outro lado, dado que $Q$ é a projeção ortogonal sobre $\operatorname{Ker}\left(\mathrm{L}_{(1, a)}+P\right)$, então $\mathrm{L}_{(1, a)}+$ $P+\varepsilon Q$ é inversível para qualquer $\varepsilon \neq 0$. Consequentemente, para $\varepsilon>0$ suficientemente pequeno,

$$
\mathrm{L}_{(0, a)}+K^{\prime}=\mathrm{L}_{(0, a)}+P+\varepsilon Q \quad \text { e } \quad \mathrm{L}_{(1, a)}+K^{\prime}=\mathrm{L}_{(1, a)}+P+\varepsilon Q
$$

são inversíveis, como queríamos provar.

Da definição de $\operatorname{sf}\left(\mathrm{L}_{(0, \cdot)}, J\right)$ e $\operatorname{sf}\left(\mathrm{L}_{(1, \cdot)}, J\right)$, temos

$$
\operatorname{sf}\left(\mathrm{L}_{(0, \cdot)}, J\right)=\operatorname{sf}\left(\mathrm{L}_{(0, \cdot)}+K^{\prime}, J\right) \quad \text { e } \quad \operatorname{sf}\left(\mathrm{L}_{(1, \cdot)}+K^{\prime}, J\right)=\operatorname{sf}\left(\mathrm{L}_{(1, \cdot)}, J\right) .
$$

Agora, tomemos a homotopia

$$
\mathrm{h}_{(s, \lambda)}=\mathrm{L}_{(s, \lambda)}+K^{\prime} \text { para } 0 \leqslant s \leqslant 1, \lambda \in J .
$$

Já que, por hipótese, $\mathrm{L}_{(s, a)}=\mathrm{L}_{(s, b)}$, de (5.2.8) se segue que os caminhos $\mathrm{h}_{(0, \cdot)}=$ $\left\{\mathrm{h}_{(0, \lambda)}\right\}_{\lambda \in J}$ e $\mathrm{h}_{(1, \cdot)}=\left\{\mathrm{h}_{(1, \lambda)}\right\}_{\lambda \in J}$ são admissíveis. Além disso,

$$
\mathrm{h}_{(s, a)}=\mathrm{L}_{(s, a)}+K^{\prime}=\mathrm{L}_{(s, b)}+K^{\prime}=\mathrm{h}_{(s, b)} \quad \text { para todo } s \in[0,1] .
$$

Logo, o Teorema 5.2.10 implica que

$$
\operatorname{sf}\left(\mathrm{h}_{(0, \cdot)}, J\right)=\operatorname{sf}\left(\mathrm{h}_{(1, \cdot)}, J\right) .
$$

Portanto, de (5.2.9) obtemos

$$
\begin{aligned}
\operatorname{sf}\left(\mathrm{L}_{(0, \cdot)}, J\right) & =\operatorname{sf}\left(\mathrm{L}_{(0, \cdot)}+K^{\prime}, J\right)=\operatorname{sf}\left(\mathrm{h}_{(0, \cdot)}, J\right)=\operatorname{sf}\left(\mathrm{h}_{(1, \cdot)}, J\right)=\operatorname{sf}\left(\mathrm{L}_{(1, \cdot)}+K^{\prime}, J\right) \\
& =\operatorname{sf}\left(\mathrm{L}_{(1, \cdot)}, J\right)
\end{aligned}
$$

o que prova a proposição. 


\subsection{Fluxo espectral para caminhos gerais de opera- dores de Fredholm auto-adjuntos}

Na seção anterior definimos o fluxo espectral para caminhos em $\Phi_{S}^{i}(H)$, onde $H$ é um espaço de Hilbert real separável de dimensão infinita. Veremos nesta seção que esta definição pode ser estendida a caminhos que estão nas outras duas componentes de $\Phi_{S}(H)$, isto é, para caminhos em $\Phi_{S}^{+}(H)$ e $\Phi_{S}^{-}(H)$, e inclusive para o caso em que $H$ tem dimensão finita. Mostraremos que o fluxo espectral para caminhos gerais em $\Phi_{S}(H)$ satisfaz as propriedades apresentadas na seção anterior. Provaremos que o fluxo espectral para um caminho $\mathrm{L}=\left\{\mathrm{L}_{\lambda}\right\}_{\lambda \in J}$ em $\Phi_{S}^{+}(H)$ coincide com a diferencia entre os índices de Morse de $\mathrm{L}_{a}$ e $\mathrm{L}_{b}$.

Nesta seção $H$ denotará um espaço de Hilbert real separável de dimensão finita ou infinita.

Para definir o fluxo espectral para caminhos em $\Phi_{S}(H)$, vejamos primeiro a seguinte construção. Fixemos um espaço de Hilbert real separável de dimensão infinita auxiliar $H_{0}$. Tomemos o espaço de Hilbert

$$
\widehat{H}=H_{0} \times H \times H_{0}
$$

dotado do o produto interno

$$
\left\langle\left(u_{1}, v_{1}, w_{1}\right),\left(u_{2}, v_{2}, w_{2}\right)\right\rangle=\left\langle u_{1}, u_{2}\right\rangle_{0}+\left\langle v_{1}, v_{2}\right\rangle+\left\langle w_{1}, w_{2}\right\rangle_{0},
$$

onde $\left(u_{1}, v_{1}, w_{1}\right),\left(u_{2}, v_{2}, w_{2}\right) \in \widehat{H}$, e $\langle\cdot, \cdot\rangle_{0}$ e $\langle\cdot, \cdot\rangle$ são os produtos internos em $H_{0}$ e $H$, respectivamente. Observe que a soma

$$
\left[H_{0} \times\{0\} \times\{0\}\right] \oplus[\{0\} \times H \times\{0\}] \oplus\left[\{0\} \times\{0\} \times H_{0}\right]
$$

é ortogonal.

A qualquer operador de Fredholm auto-adjunto $L \in L(H)$ associamos o operador $\widehat{L}: \widehat{H} \rightarrow \widehat{H}$ definido por

$$
\widehat{L}(u, v, w)=(u, L(v),-w)
$$

isto é,

$$
\widehat{L}=\left(I_{H_{0}}, L,-I_{H_{0}}\right) .
$$

Não é difícil provar que $\widehat{L}$ é limitado.

De forma análoga à prova da igualdade dada em (3.2.1) podemos provar que

$$
\widehat{L}^{*}=\left(I_{H_{0}}, L^{*},-I_{H_{0}}\right) \text {. }
$$

Assim, dado que $L$ é auto-adjunto, $\widehat{L}$ é auto-adjunto.

Por outro lado, como resultado análogo ao Lema 4.2.9, temos

$H_{+}(\widehat{L})=H_{0} \times H_{+}(L) \times\{0\}, H_{-}(\widehat{L})=\{0\} \times H_{-}(L) \times H_{0}$ e $\operatorname{Ker} \widehat{L}=\{0\} \times \operatorname{Ker} L \times\{0\}$. 
Daí,

$$
\widehat{H}=H_{+}(\widehat{L}) \oplus H_{-}(\widehat{L}) \oplus \operatorname{Ker} \widehat{L} .
$$

Este fato prova que $\widehat{L}$ é um operador de Fredholm auto-adjunto fortemente indefinido.

Com base na construção feita acima, obtemos a seguinte definição.

Definição 5.3.1 (Fluxo espectral para caminhos em $\Phi_{S}(H)$ ). Seja $\mathbf{L}=\left\{\mathbf{L}_{\lambda}\right\}_{\lambda \in J}$ um caminho admissível ou fechado em $\Phi_{S}(H)$. Para cada $\mathbf{L}_{\lambda}$, consideremos o operador associado $\widehat{L}_{\lambda}$ costruído acima. O fluxo espectral de $\mathrm{L}$ é definido como o fluxo espectral do caminho $\left\{\widehat{\mathrm{L}}_{\lambda}\right\}_{\lambda \in I} \subseteq \Phi_{S}^{i}(\widehat{H})$, isto é,

$$
\operatorname{sf}(\mathrm{L}, J)=\operatorname{sf}(\widehat{\mathrm{L}}, J) .
$$

No resto desta seção $L=\left\{L_{\lambda}\right\}_{\lambda \in J}$ denotará um caminho admissível ou fechado de operadores em $\Phi_{S}(H)$.

Vejamos que, se $H$ tem dimensão infinita e L é um caminho em $\Phi_{S}^{i}(H)$, esta nova definição coincide com a dada na Definição 5.2.1. De fato, a Proposição 5.2.4 implica que

$$
\operatorname{sf}\left(\left\{I_{H_{0}}\right\}_{\lambda \in J}, J\right)=\operatorname{sf}\left(\left\{-I_{H_{0}}\right\}_{\lambda \in J}, J\right)=0 .
$$

Além disso, de (5.3.1) e da propriedade do fluxo espectral do produto direto (Proposição 5.2.8), se segue que

$$
\operatorname{sf}(\widehat{\mathrm{L}}, J)=\operatorname{sf}\left(\left\{I_{H_{0}}\right\}_{\lambda \in J}, J\right)+\operatorname{sf}(\mathrm{L}, J)+\operatorname{sf}\left(\left\{-I_{H_{0}}\right\}_{\lambda \in J}, J\right)=\operatorname{sf}(\mathrm{L}, J),
$$

como queríamos provar.

Mostremos agora que a fórmula (5.2.6) é também válida para o caminho $\mathrm{L}$, sempre que $\mathrm{L}_{\lambda}-\mathrm{L}_{a}$ seja compacto para todo $\lambda \in J$.

Proposição 5.3.2. Suponhamos que, para o caminho $L$, o operador $L_{\lambda}-L_{a}$ seja compacto para todo $\lambda \in J$. Então,

$$
\operatorname{sf}(L, J)=\mu_{r e l}\left(L_{a}, L_{b}\right) .
$$

Demonstração. Primeiro, observe que $\widehat{\mathrm{L}}_{\lambda}-\widehat{\mathrm{L}}_{a} \in L(\widehat{H})$ também é compacto para todo $\lambda \in J$, pois

$$
\widehat{\mathrm{L}}_{\lambda}-\widehat{\mathrm{L}}_{a}=\left(I_{H_{0}}-I_{H_{0}}, \mathrm{~L}_{\lambda}-\mathrm{L}_{a},-I_{H_{0}}+I_{H_{0}}\right)=\left(0, \mathrm{~L}_{\lambda}-\mathrm{L}_{a}, 0\right) .
$$

Consequentemente, da Proposição 5.2.6 temos

$$
\operatorname{sf}(\widehat{\mathrm{L}}, J)=\mu_{r e l}\left(\widehat{\mathrm{L}}_{a}, \widehat{\mathrm{L}}_{b}\right) .
$$


Vejamos que $\mu_{\text {rel }}\left(\widehat{\mathrm{L}}_{a}, \widehat{\mathrm{L}}_{b}\right)=\mu_{\text {rel }}\left(\mathrm{L}_{a}, \mathrm{~L}_{b}\right)$. Dado que $H_{-}\left(\widehat{\mathrm{L}}_{\lambda}\right)=\{0\} \times H_{-}\left(\mathrm{L}_{\lambda}\right) \times H_{0}$ para $\lambda=a, b$, então $\left[H_{-}\left(\widehat{\mathrm{L}}_{\lambda}\right)\right]^{\perp}=H_{0} \times\left[H_{-}\left(\mathrm{L}_{\lambda}\right)\right]^{\perp} \times\{0\}$. Assim,

$$
\begin{aligned}
H_{-}\left(\widehat{\mathrm{L}}_{a}\right) \cap\left[H_{-}\left(\widehat{\mathrm{L}}_{b}\right)\right]^{\perp} & =\left[\{0\} \times H_{-}\left(\mathrm{L}_{a}\right) \times H_{0}\right] \cap\left[H_{0} \times\left[H_{-}\left(\mathrm{L}_{b}\right)\right]^{\perp} \times\{0\}\right] \\
& =\left[\{0\} \cap H_{0}\right] \times\left[H_{-}\left(\mathrm{L}_{a}\right) \cap\left[H_{-}\left(\mathrm{L}_{b}\right)\right]^{\perp}\right] \times\left[H_{0} \cap\{0\}\right] \\
& =\{0\} \times\left[H_{-}\left(\mathrm{L}_{a}\right) \cap\left[H_{-}\left(\mathrm{L}_{b}\right)\right]^{\perp}\right] \times\{0\} .
\end{aligned}
$$

$\operatorname{Logo}, \operatorname{dim}\left(H_{-}\left(\widehat{\mathrm{L}}_{a}\right) \cap\left[H_{-}\left(\widehat{\mathrm{L}}_{b}\right)\right]^{\perp}\right)=\operatorname{dim}\left(H_{-}\left(\mathrm{L}_{a}\right) \cap\left[H_{-}\left(\mathrm{L}_{b}\right)\right]^{\perp}\right)$. Analogamente,

$$
H_{-}\left(\widehat{\mathrm{L}}_{b}\right) \cap\left[H_{-}\left(\widehat{\mathrm{L}}_{a}\right)\right]^{\perp}=\{0\} \times\left[H_{-}\left(\mathrm{L}_{b}\right) \cap\left[H_{-}\left(\mathrm{L}_{a}\right)\right]^{\perp}\right] \times\{0\} .
$$

Portanto, $\operatorname{dim}\left(H_{-}\left(\widehat{\mathrm{L}}_{b}\right) \cap\left[H_{-}\left(\widehat{\mathrm{L}}_{a}\right)\right]^{\perp}\right)=\operatorname{dim}\left(H_{-}\left(\mathrm{L}_{b}\right) \cap\left[H_{-}\left(\mathrm{L}_{a}\right)\right]^{\perp}\right)$. Daí,

$$
\begin{aligned}
\mu_{r e l}\left(\widehat{\mathrm{L}}_{a}, \widehat{\mathrm{L}}_{b}\right) & =\operatorname{dim}\left(H_{-}\left(\widehat{\mathrm{L}}_{a}\right) \cap\left[H_{-}\left(\widehat{\mathrm{L}}_{b}\right)\right]^{\perp}\right)-\operatorname{dim}\left(H_{-}\left(\widehat{\mathrm{L}}_{b}\right) \cap\left[H_{-}\left(\widehat{\mathrm{L}}_{a}\right)\right]^{\perp}\right) \\
& =\operatorname{dim}\left(H_{-}\left(\mathrm{L}_{a}\right) \cap\left[H_{-}\left(\mathrm{L}_{b}\right)\right]^{\perp}\right)-\operatorname{dim}\left(H_{-}\left(\mathrm{L}_{b}\right) \cap\left[H_{-}\left(\mathrm{L}_{a}\right)\right]^{\perp}\right) \\
& =\mu_{\text {rel }}\left(\mathrm{L}_{a}, \mathrm{~L}_{b}\right) .
\end{aligned}
$$

Consequentemente, a definição $\operatorname{de} \operatorname{sf}(\mathrm{L}, J)=\operatorname{sf}(\widehat{\mathrm{L}}, J)$ e a fórmula (5.3.3) implicam que

$$
\operatorname{sf}(\mathrm{L}, J)=\mu_{r e l}\left(\mathrm{~L}_{a}, \mathrm{~L}_{b}\right)
$$

o que prova a proposição.

As demais propriedades do fluxo espectral que foram provadas na seção anterior para caminhos em $\Phi_{S}^{i}(H)$, se podem mostrar de forma análoga para caminhos em $\Phi_{S}(H)$.

No seguinte teorema mostraremos que o fluxo espectral para caminhos admissíveis de operadores essencialmente positivos coincide com a diferença dos índices de Morse dos extremos do caminho.

Teorema 5.3.3. Se L é um caminho admissível de operadores essencialmente positivos, então

$$
\operatorname{sf}(L, J)=\mu\left(L_{a}\right)-\mu\left(L_{b}\right) .
$$

Demonstração. Primeiro provemos que, se $S, T$ e $R$ são isomorfismos essencialmente positivos e congruentes, então

$$
\mu_{\text {rel }}(S, T)=\mu_{\text {rel }}(S, R)+\mu_{r e l}(R, T) .
$$

De fato, tomemos $R=S+K_{1}$ e $T=R+K_{2}$, onde $K_{1}$ e $K_{2}$ pertencem a $K_{S}(H)$. A convexidade de $\Phi_{S}^{+}(H)$ implica que $S+\lambda K_{1} \in \Phi_{S}^{+}(H)$ para $0 \leq \lambda \leq 1$ e $R+(\lambda-1) K_{2} \in$ $\Phi_{S}^{+}(H)$ para $1 \leq \lambda \leq 2$. Seja $\mathrm{M}:[0,2] \rightarrow \Phi_{S}^{+}(H)$ definido por

$$
\mathrm{M}_{\lambda}=\left\{\begin{array}{cc}
S+\lambda K_{1} & \text { se } 0 \leq \lambda \leq 1 \\
R+(\lambda-1) K_{2} & \text { se } 1 \leq \lambda \leq 2
\end{array}\right.
$$


Então, $M$ é um caminho de operadores de Fredholm essencialmente positivos congruentes, tal que $\mathrm{M}_{0}=S, \mathrm{M}_{1}=R$ e $\mathrm{M}_{2}=T$ são inversíveis. Assim, aplicando a Proposição 5.2.7 para caminhos gerais temos

$$
\operatorname{sf}(\mathrm{M},[0,2])=\operatorname{sf}(\mathrm{M},[0,1])+\operatorname{sf}(\mathrm{M},[1,2]) .
$$

Logo, como M é um caminho de operadores congruentes, a fórmula (5.3.2) implica que

$$
\mu_{\text {rel }}(S, T)=\mu_{\text {rel }}(S, R)+\mu_{\text {rel }}(R, T) .
$$

Além disso, de (4.4.12) temos que, se $T$ é definido positivo, então

$$
\mu_{r e l}(S, T)=\mu_{r e l}\left(T-\left(K_{1}+K_{2}\right), T\right)=\mu\left(T-\left(K_{1}+K_{2}\right)\right)=\mu(S) .
$$

Agora, consideremos os seguintes casos:

Caso (i). Suponhamos que os operadores do caminho sejam congruentes. Então, de (5.3.2) temos

$$
\operatorname{sf}(\mathrm{L}, J)=\mu_{r e l}\left(\mathrm{~L}_{a}, \mathrm{~L}_{b}\right)
$$

Provemos que, se $s>\left\|\mathrm{L}_{a}\right\|$ e se $K$ é a projeção ortogonal sobre $H_{-}\left(\mathrm{L}_{a}\right) \oplus \mathrm{Ker} \mathrm{L}_{a}$, então $T=\mathrm{L}_{a}+s K$ é um isomorfismo definido positivo congruente a $\mathrm{L}_{a}$. De fato, é claro que $s K$ é definida positiva em Ker $\mathrm{L}_{a}$. Portanto, dado que $T$ coincide com $\mathrm{L}_{a}$ em $H_{+}\left(\mathrm{L}_{a}\right)$ e com $s K$ em Ker $\mathrm{L}_{a}, T$ é definido positivo em $H_{+}\left(\mathrm{L}_{a}\right) \oplus \operatorname{Ker} \mathrm{L}_{a}$. Agora, como $s>\left\|\mathrm{L}_{a}\right\|$, temos

$$
\left\langle-\mathrm{L}_{a} x, x\right\rangle \leq\left\|\mathrm{L}_{a}\right\|\|x\|^{2}<s\|x\|^{2}=\langle s x, x\rangle \quad \text { para } x \in H \text { com } x \neq 0 .
$$

Assim, $\mathrm{L}_{a}+s I$ é definido positivo.

Observe que $\mathrm{L}_{a}+s I$ coincide com $T$ em $H_{-}\left(\mathrm{L}_{a}\right)$, pois

$$
\left(\mathrm{L}_{a}+s I\right) x=\mathrm{L}_{a} x+s x=\mathrm{L}_{a} x+s K x=T x \quad \text { para todo } x \in H_{-}\left(\mathrm{L}_{a}\right) .
$$

Logo, $T$ é definido positivo em $H_{-}\left(\mathrm{L}_{a}\right)$. Os fatos acima mostram que $T$ é definido positivo para $s>\left\|\mathrm{L}_{a}\right\|$.

Por outro lado, dado que $T$ é de Fredholm auto-adjunto com $\operatorname{Ker} T=\{0\}$, a Proposição 3.4.1 implica que $T$ é um isomorfismo. Em conclusão, $T$ é um isomorfismo definido positivo congruente a $\mathrm{L}_{a}$.

Como, por hipótese, $\mathrm{L}_{a}$ e $\mathrm{L}_{b}$ são congruentes, então $T$ é congruente $a \mathrm{~L}_{b}$. De (5.3.4) e (5.3.5) temos

$$
\begin{aligned}
\operatorname{sf}(\mathrm{L}, J)=\mu_{r e l}\left(\mathrm{~L}_{a}, \mathrm{~L}_{b}\right) & =\mu_{r e l}\left(\mathrm{~L}_{a}, T\right)+\mu_{r e l}\left(T, \mathrm{~L}_{b}\right) \\
& =\mu_{r e l}\left(\mathrm{~L}_{a}, T\right)-\mu_{r e l}\left(\mathrm{~L}_{b}, T\right) \\
& =\mu\left(\mathrm{L}_{a}\right)-\mu\left(\mathrm{L}_{b}\right) .
\end{aligned}
$$


Assim, no primeiro caso a proposição é provada.

Caso(ii). Suponhamos que os extremos do caminho sejam isomorfismos definidos positivos. Neste caso devemos provar que $\operatorname{sf}(\mathrm{L}, I)=0$, pois $\mu\left(\mathrm{L}_{a}\right)=\mu\left(\mathrm{L}_{b}\right)=0$. Consideremos a homotopia

$$
\mathrm{h}(s, \lambda)=s \mathrm{~L}_{\lambda}+(1-s) I, \quad 0 \leq s \leq 1, \quad a \leq \lambda \leq b .
$$

Da convexidade de $\Phi_{S}^{+}(H)$ temos que $\mathrm{h}(s, \lambda) \in \Phi_{S}^{+}(H)$ para $0 \leq s \leq 1, a \leq \lambda \leq b$. Além disso, dado que o conjunto dos isomorfismos auto-adjuntos definidos positivos é convexo (Corolário 3.4.8), se segue que, para cada $s \in[0,1]$,

$$
\mathrm{h}(s, a)=s \mathrm{~L}_{a}+(1-s) I \quad \text { e } \quad \mathrm{h}(s, b)=s \mathrm{~L}_{b}+(1-s) I
$$

são isomorfismos definidos positivos ( $\mathrm{L}_{a}$ e $\mathrm{L}_{b}$ são isomorfismos definidos positivos por hipótese). Assim, h é uma homotopia de caminhos admissíveis entre $\mathrm{L}$ e o caminho constante $\{I\}_{\lambda \in J}$. Pela Proposição 5.2.4 e o Teorema 5.2.9, temos

$$
\operatorname{sf}(\mathrm{L}, J)=\operatorname{sf}\left(\{I\}_{\lambda \in J}, J\right)=0 .
$$

Finalmente consideramos o caso geral. Como se provou acima, existem operadores auto-adjuntos compactos $K_{a}$ e $K_{b}$ tais que $\mathrm{L}_{a}+K_{a}$ e $\mathrm{L}_{b}+K_{b}$ são isomorfismos definidos positivos. Para $0 \leq t \leq 1$, definamos

$$
A_{t}=\mathrm{L}_{a}+(1-t) K_{a} \quad \text { e } \quad B_{t}=\mathrm{L}_{b}+t K_{b} .
$$

Pela convexidade de $\Phi_{S}^{+}(H)$, temos que $A_{t}$ e $B_{t}$ pertencem a $\Phi_{S}^{+}(H)$ para $0 \leq t \leq 1$. É claro que $A=\left\{A_{t}\right\}_{t \in[0,1]}$ e $B=\left\{B_{t}\right\}_{t \in[0,1]}$ são caminhos de operadores congruentes. Portanto, do primeiro caso se segue

$$
\operatorname{sf}(A,[0,1])=\mu\left(A_{0}\right)-\mu\left(A_{1}\right)=\mu\left(\mathrm{L}_{a}+K_{a}\right)-\mu\left(\mathrm{L}_{a}\right)
$$

e

$$
\operatorname{sf}(B,[0,1])=\mu\left(B_{0}\right)-\mu\left(B_{1}\right)=\mu\left(\mathrm{L}_{b}\right)-\mu\left(\mathrm{L}_{b}+K_{b}\right) .
$$

Seja $C:[0,1] \rightarrow \Phi_{S}^{+}(H)$ definido por

$$
C_{t}=\left\{\begin{array}{cc}
A_{3 t} & \text { se } 0 \leq t \leq 1 / 3 \\
\mathrm{~L}_{(3 t-1) b+(2-3 t) a} & \text { se } 1 / 3 \leq t \leq 2 / 3 \\
B_{3 t-2} & \text { se } 2 / 3 \leq t \leq 1
\end{array}\right.
$$

Então, $C$ é contínua $t$ e

$$
C_{0}=\mathrm{L}_{a}+K_{a}, \quad C_{1 / 3}=\mathrm{L}_{a}, \quad C_{2 / 3}=\mathrm{L}_{b} \quad \text { e } \quad C_{1}=\mathrm{L}_{b}+K_{b},
$$


isto é, $C$ é um caminho em $\Phi_{S}^{+}(H)$ com extremos definidos positivos. Logo, pelo segundo caso considerado, seu fluxo espectral é zero. Pela aditividade do fluxo espectral, temos que

$$
\begin{aligned}
0 & =\operatorname{sf}(C,[0,1]) \\
& =\operatorname{sf}(C,[0,1 / 3])+\operatorname{sf}(C,[1 / 3,2 / 3])+\operatorname{sf}(B,[2 / 3,1]) \\
& =\operatorname{sf}(A,[0,1])+\operatorname{sf}(\mathrm{L},[a, b])+\operatorname{sf}(B,[0,1]) .
\end{aligned}
$$

Agora, dado que $\mu\left(\mathrm{L}_{a}+K_{a}\right)=\mu\left(\mathrm{L}_{b}+K_{b}\right)=0$, de (5.3.6) e (5.3.7) se segue

$$
\begin{aligned}
\operatorname{sf}(\mathrm{L},[a, b]) & =-\operatorname{sf}(A,[0,1])-\operatorname{sf}(B,[0,1]) \\
& =\mu\left(\mathrm{L}_{a}\right)-\mu\left(\mathrm{L}_{a}+K_{a}\right)-\left(\mu\left(\mathrm{L}_{b}\right)-\mu\left(\mathrm{L}_{b}+K_{b}\right)\right) \\
& =\mu\left(\mathrm{L}_{a}\right)-\mu\left(\mathrm{L}_{b}\right),
\end{aligned}
$$

o que prova o teorema.

Como consequência do teorema anterior temos o seguinte corolário.

Corolário 5.3.4. Se L é um caminho admissivel de operadores essencialmente negativos, então

$$
\operatorname{sf}(L, J)=\mu\left(-L_{b}\right)-\mu\left(-L_{a}\right) .
$$

Demonstração. De fato, é fácil ver que, para um caminho admissível T de operadores de operadores de Fredholm auto-adjuntos fortemente indefinidos,

$$
\operatorname{sf}(\mathrm{T}, J)=-\operatorname{sf}(-\mathrm{T}, J) .
$$

Agora, dado que $\left\{-L_{\lambda}\right\}_{\lambda \in J}$ é um caminho admissível de operadores de Fredholm autoadjuntos essencialmente positivos, se segue da teorema anterior que

$$
\operatorname{sf}(-\mathrm{L}, J)=\mu\left(-\mathrm{L}_{a}\right)-\mu\left(-\mathrm{L}_{b}\right) .
$$

Consequentemente,

$$
\operatorname{sf}(\mathrm{L}, J)=-\operatorname{sf}(-\mathrm{L}, J)=-\left(\mu\left(-\mathrm{L}_{a}\right)-\mu\left(-\mathrm{L}_{b}\right)\right)=\mu\left(-\mathrm{L}_{b}\right)-\mu\left(-\mathrm{L}_{a}\right),
$$

isto é, $\operatorname{sf}(\mathrm{L}, J)=\mu\left(-\mathrm{L}_{b}\right)-\mu\left(-\mathrm{L}_{a}\right)$.

Por último vejamos o caso em que $H$ tem dimensão finita.

Proposição 5.3.5. Se L é um caminho de operadores auto-adjuntos em um espaço de Hilbert de dimensão finita $H$, então

$$
\operatorname{sf}(L, J)=\mu\left(L_{a}\right)-\mu\left(L_{b}\right) .
$$


Demonstração. É claro que L é um caminho de operadores dois a dois congruentes. Consequentemente, da Proposição 5.3.2 obtemos

$$
\operatorname{sf}(\mathrm{L}, J)=\mu_{r e l}\left(\mathrm{~L}_{a}, \mathrm{~L}_{b}\right) .
$$

Como $H_{-}\left(\mathrm{L}_{a}\right)$ e $H_{-}\left(\mathrm{L}_{b}\right)$ têm dimensão finita, do Lema 4.4 .8 se segue

$$
\mu_{r e l}\left(\mathrm{~L}_{a}, \mathrm{~L}_{b}\right)=\operatorname{dim}\left(H_{-}\left(\mathrm{L}_{a}\right) \cap H_{-}\left(\mathrm{L}_{b}\right)^{\perp}\right)-\operatorname{dim}\left(H_{-}\left(\mathrm{L}_{b}\right) \cap H_{-}\left(\mathrm{L}_{a}\right)^{\perp}\right)=\mu\left(\mathrm{L}_{a}\right)-\mu\left(\mathrm{L}_{b}\right) .
$$

Em conclusão, $\operatorname{sf}(\mathrm{L}, J)=\mu\left(\mathrm{L}_{a}\right)-\mu\left(\mathrm{L}_{b}\right)$.

\subsection{Fluxo espectral em pontos singulares isolados}

Para um caminho de operadores de Fredholm auto-adjuntos $L=\left\{L_{\lambda}\right\}_{\lambda \in J}$, o conjunto singular de L é definido como

$$
\Sigma(\mathrm{L})=\left\{\lambda \in J: \mathrm{L}_{\lambda} \text { não é inversível }\right\} .
$$

Nesta última parte do trabalho definiremos o fluxo espectral para caminhos de operadores de Fredholm auto-adjuntos em um ponto $\lambda_{0} \in J$ que seja isolado no seu conjunto singular.

Dado um caminho $\mathrm{L}$ em $\Phi_{S}(H)$, consideremos um ponto interior $\lambda_{0}$ de $J$ isolado em $\Sigma(\mathrm{L})$. Para este ponto singular isolado definimos o fluxo espectral de $L$ através de $\lambda_{0}$ por

$$
\operatorname{sf}\left(\mathrm{L}, \lambda_{0}\right)=\lim _{\delta \rightarrow 0} \operatorname{sf}\left(\mathrm{L},\left[\lambda_{0}-\delta, \lambda_{0}+\delta\right]\right) .
$$

Observe que este limite existe sempre que $\lambda_{0}$ seja um ponto interior de $J$ e isolado em $\Sigma(\mathrm{L})$. De fato, se $\lambda_{0}$ é isolado em $\Sigma(\mathrm{L})$, existe $\varepsilon>0$ tal que $\mathrm{L}_{\lambda}$ é inversível para todo $\lambda \in\left[\lambda_{0}-\varepsilon, \lambda_{0}+\varepsilon\right]$ e $\lambda \neq \lambda_{0}$. Assim, para qualquer $0<\delta<\varepsilon$, o caminho $\mathrm{L}$ é admissível nos intervalos $\left[\lambda_{0}-\varepsilon, \lambda_{0}-\delta\right],\left[\lambda_{0}-\delta, \lambda_{0}+\delta\right]$ e $\left[\lambda_{0}+\delta, \lambda_{0}+\varepsilon\right]$, pois $\mathrm{L}_{\lambda}$ é inversível para $\lambda \in\left\{\lambda_{0}-\varepsilon, \lambda_{0}-\varepsilon, \lambda_{0}+\delta, \lambda_{0}-\varepsilon\right\}$. Se segue da Proposição 5.2.7 que

$$
\begin{aligned}
\operatorname{sf}\left(\mathrm{L},\left[\lambda_{0}-\varepsilon, \lambda_{0}+\varepsilon\right]\right) & \\
\quad & \operatorname{sf}\left(\mathrm{L},\left[\lambda_{0}-\varepsilon, \lambda_{0}-\delta\right]\right)+\operatorname{sf}\left(\mathrm{L},\left[\lambda_{0}-\delta, \lambda_{0}+\delta\right]\right)+\operatorname{sf}\left(\mathrm{L},\left[\lambda_{0}+\delta, \lambda_{0}+\varepsilon\right]\right) .
\end{aligned}
$$

Como $L_{\lambda}$ é inversível para todo $\lambda$ em $\left[\lambda_{0}-\varepsilon, \lambda_{0}-\delta\right] \cup\left[\lambda_{0}+\delta, \lambda_{0}+\varepsilon\right]$, da propriedade de normalização do fluxo espectral (Proposição 5.2.4), temos que

$$
\operatorname{sf}\left(\mathrm{L},\left[\lambda_{0}-\varepsilon, \lambda_{0}-\delta\right]\right)=\operatorname{sf}\left(\mathrm{L},\left[\lambda_{0}+\delta, \lambda_{0}+\varepsilon\right]\right)=0 .
$$

Assim, $\operatorname{sf}\left(\mathrm{L},\left[\lambda_{0}-\varepsilon, \lambda_{0}+\varepsilon\right]\right)=\operatorname{sf}\left(\mathrm{L},\left[\lambda_{0}-\delta, \lambda_{0}+\delta\right]\right)$ para todo $0<\delta<\varepsilon$. Este fato prova a existência do limite em (5.4.1).

Veremos algumas propriedades que possui o fluxo espectral um ponto $\lambda_{0}$ de $\Sigma(\mathrm{L})$ quando o caminho L é continuamente diferenciável. Para este fim, primeiro vejamos os seguintes conceitos. 
Definição 5.4.1. Sejam $E$ e $F$ espaços de Banach e $U$ um subconjunto aberto de $E$. Consideremos uma aplicação $f: U \rightarrow F$ e $x_{0} \in U$. Dizemos que que $f$ é diferenciável $^{1}$ em $x_{0}$ se existem um operador linear contínuo $M_{x_{0}}: E \rightarrow F$ e uma aplicação $r: U_{0} \rightarrow F$ definida em uma vizinhaça suficientemente pequena $U_{0}$ de $0 \in E$, com valores em $F$, tais que, para $h \in E$, com norma suficientemente pequena,

$$
f\left(x_{0}+h\right)-f\left(x_{0}\right)=M_{x_{0}}(h)+r(h) \quad \text { e } \quad \lim _{h \rightarrow 0} \frac{r(h)}{\|h\|}=0 .
$$

Denotaremos por $f^{\prime}\left(x_{0}\right)$ o operador $M_{x_{0}}$ e o chamaremos de diferencial de $f$ no ponto $x_{0}$. Dizemos que $f$ é de classe $C^{1}$, ou continuamente diferenciável, se $f^{\prime}(x)$ existe para todo $x \in U$ e a aplicação $f^{\prime}: U \rightarrow L(E, F)$ definida por $x \mapsto f^{\prime}(x)$ é contínua.

Podemos ver uma apresentação detalhada da noção anterior e das suas propriedades em, por exemplo, [18], Capítulo XIII, § 2.

Observação 5.4.2. Seja $f: J \rightarrow F$ um caminho contínuamente diferenciável. Então, dado que seu diferencial $f^{\prime}\left(\lambda_{0}\right): \mathbb{R} \rightarrow F$ em um ponto interior $\lambda_{0}$ de $J$ é um operador linear de $\mathbb{R}$ em $F$, obtemos que

$$
f^{\prime}\left(\lambda_{0}\right)(\lambda)=\lambda f^{\prime}\left(\lambda_{0}\right)(1) \quad \text { para todo } \lambda \in \mathbb{R} .
$$

Assim, abusando um pouco da notação, podemos identificar o operador $f^{\prime}\left(\lambda_{0}\right)$ com o elemento $f^{\prime}\left(\lambda_{0}\right)(1)$ de $F$.

Definição 5.4.3. O operador $f^{\prime}\left(\lambda_{0}\right)(1)$ da observação anterior será denotado por $\dot{f}\left(\lambda_{0}\right)$.

No resto do trabalho, $\mathrm{L}=\left\{\mathrm{L}_{\lambda}\right\}_{\lambda \in J} \subseteq \Phi_{S}(H)$ denotará um caminho continuamente diferenciável.

Definição 5.4.4. A um ponto $\lambda_{0}$ de $\Sigma(\mathrm{L})$ associamos a forma quadrática $Q\left(\mathrm{~L}, \lambda_{0}\right)$ no $\operatorname{Ker} \mathrm{L}_{\lambda_{0}}$ definida pela restrição da forma $\left\langle\dot{\mathrm{L}}_{\lambda_{0}} h, h\right\rangle$ a $\operatorname{Ker} \mathrm{L}_{\lambda_{0}}$, isto é,

$$
Q\left(\mathrm{~L}, \lambda_{0}\right)(h)=\left\langle\dot{\mathrm{L}}_{\lambda_{0}} h, h\right\rangle \text { para } h \in \operatorname{Ker} \mathrm{L}_{\lambda_{0}} .
$$

A forma $Q\left(\mathrm{~L}, \lambda_{0}\right)$ será chamada de forma crossing de $\mathrm{L}$ em $\lambda_{0}$.

Seja $\lambda_{0}$ um ponto de $\Sigma(\mathrm{L})$. Dizemos $Q\left(\mathrm{~L}, \lambda_{0}\right)$ é não degenerada se a restrição $\left.P \dot{\mathrm{L}}_{\lambda_{0}} P\right|_{\text {Ker } \mathrm{L}_{\lambda_{0}}}$ é injetora, onde $P$ é a projeção ortogonal sobre o núcleo de $\mathrm{L}_{\lambda_{0}}$. Se $\lambda_{0}$ é isolado em $\Sigma(\mathrm{L})$ e $Q\left(\mathrm{~L}, \lambda_{0}\right)$ é não degenerada, $\lambda_{0}$ será chamado de ponto singular regular de $\mathrm{L}$.

Finalizaremos o trabalho provando que, se $\lambda_{0}$ é um ponto singular regular de L, então

$$
\operatorname{sf}\left(\mathrm{L}, \lambda_{0}\right)=\operatorname{sign} Q\left(\mathrm{~L}, \lambda_{0}\right)=\operatorname{sign}\left(\left.P \dot{\mathrm{L}}_{\lambda_{0}} P\right|_{\operatorname{Ker}_{\mathrm{L}_{0}}}\right) .
$$

Para este fim, primeiro mostraremos a diferenciabilidade de algumas aplicações que serão consideradas nesta prova.

\footnotetext{
${ }^{1}$ É o clássico conceito de diferenciabilidade segundo Fréchet.
} 
Teorema 5.4.5. A aplicação

$$
\begin{aligned}
\mathcal{L}: G L(H) & \rightarrow G L(H) \\
L & \mapsto L^{-1}
\end{aligned}
$$

é continuamente diferenciável.

Demonstração. Fixemos $L \in G L(H)$. Da continuidade de $\mathcal{L}$, que foi provada no Lema 1.1.9, temos que, para $T \in L(H)$ com norma suficientemente pequena, o operador $(L+T)^{-1}$ existe. É claro que $T \mapsto-L^{-1} T L^{-1}$ define um operador linear limitado de $L(H)$ em $L(H)$. Tomemos $r: L(H) \rightarrow L(H)$ dado por

$$
r(T)=L^{-1} T L^{-1}+\mathcal{L}(L+T)-\mathcal{L}(L)
$$

e mostremos que

$$
\lim _{T \rightarrow 0} \frac{r(T)}{\|T\|}=0
$$

De fato, de (1.1.1) obtemos que

$$
\mathcal{L}(L+T)-\mathcal{L}(L)=(L+T)^{-1}-L^{-1}=-(L+T)^{-1} T L^{-1} .
$$

Assim,

$$
\begin{aligned}
\frac{\|r(T)\|}{\|T\|} & =\frac{\left\|L^{-1} T L^{-1}-(L+T)^{-1} T L^{-1}\right\|}{\|T\|} \leq \frac{\left\|L^{-1}-(L+T)^{-1}\right\|\|T\|\left\|L^{-1}\right\|}{\|T\|} \\
& =\left\|L^{-1}-(L+T)^{-1}\right\|,
\end{aligned}
$$

o que mostra o limite em (5.4.4). Os fatos acima provam que $\mathcal{L}$ é diferenciável em $L$ e que $\mathcal{L}^{\prime}(L)(T)=-L^{-1} T L^{-1}$ para $T \in L(H)$. Não é difícil provar que $L \mapsto$ $\mathcal{L}^{\prime}(L)$, para $L \in G L(H)$, é uma aplicacão contínua. Em conclusão, $\mathcal{L}$ é continuamente diferenciável.

Considerando $L_{S}(H)$ como subespaço topológico de $L(H), G L_{S}^{+}(H)$ é um subconjunto aberto de $L_{S}(H)$ (Proposição 3.4.9). Provaremos que existe uma vizinhança $U_{0} \subseteq G L_{S}^{+}(H)$ do operador identidade tal que a aplicação raiz quadrada é continuamente diferenciável em $U_{0}$. Para provar este fato, precisaremos do Teorema da aplicação inversa, cuja prova se pode ver, por exemplo, em [18], pág. 361, Teorema 1.2. Antes de apresentar o Teorema da aplicação inversa vejamos a seguinte definição.

Definição 5.4.6. Suponhamos que $E$ e $F$ sejam espaços de Banach e $U$ seja um subconjunto aberto de $E$. Seja $f: U \rightarrow F$ uma aplicação continuamente diferenciável. Dizemos que $f$ é um isomorfismo $C^{1}$ sobre a imagem $f(U)$, se $f(U)$ é um subconjunto aberto de $F$ e se existe uma aplicação $g: f(U) \rightarrow U$ continuamente diferenciável tal que $g \circ f$ e $f \circ g$ são as aplicações identidades em $U$ e $f(U)$, respectivamente. Dizemos que $f: U \rightarrow F$ é um difeomorfismo local $C^{1}$ em um ponto $x_{0} \in U$ se existe um subconjunto aberto $U_{0} \subseteq U$ tal que $x_{0} \in U_{0}$ e a restrição de $f$ a $U_{0}$ é um isomorfismo continuamente diferenciável. 
Para o seguinte teorema, $E$ e $F$ denotarão espaços de Banach.

Teorema 5.4.7 (Teorema da aplicação inversa). Seja $U$ um subconjunto aberto de $E$ e seja $f: U \rightarrow F$ uma aplicação continuamente diferenciável. Tomemos $x_{0} \in U e$ assumamos que $f^{\prime}\left(x_{0}\right) \in L(E, F)$ seja um isomorfismo. Então, $f$ é um difeomorfismo local $C^{1}$ em $x_{0}$.

Lema 5.4.8. A aplicação

$$
\begin{aligned}
\mathcal{C}: G L_{S}^{+}(H) & \rightarrow G L_{S}^{+}(H) \\
L & \mapsto L^{2}
\end{aligned}
$$

é continuamente diferenciável e, além disso,

$$
\mathcal{C}^{\prime}(L) T=L T+T L
$$

$\operatorname{para} L \in G L_{S}^{+}(H)$ e $T \in L_{S}(H)$.

Demonstração. É claro que $\mathcal{C}$ é contínua. Fixemos $L \in G L_{S}^{+}(H)$. É claro que $T \mapsto$ $L T+T L$, para $T \in L_{S}(H)$, define um operador linear contínuo de $L_{S}(H)$ em $L_{S}(H)$. Agora, tomando $r(T)=T^{2}$ para $T \in L_{S}(H)$, obtemos que

$$
\lim _{T \rightarrow 0} \frac{r(T)}{\|T\|}=0
$$

e

$$
\mathcal{C}(L+T)-\mathcal{C}(L)=(L+T)^{2}-L^{2}=L^{2}+L T+T L+T^{2}-L^{2}=L T+T L+r(T) .
$$

Portanto, $\mathcal{C}^{\prime}(L) T=L T+T L$ para $L \in G L_{S}^{+}(H)$ e $T \in L_{S}(H)$. É fácil ver que $L \mapsto \mathcal{C}^{\prime}(L)$ é contínua. Assim, $\mathcal{C}$ é continuamente diferenciável.

Como consequência do Teorema da aplicação inversa e do lema anterior temos a seguinte proposição.

Proposição 5.4.9. A aplicação

$$
\begin{aligned}
\mathcal{R}: G L_{S}^{+}(H) & \rightarrow G L_{S}^{+}(H) \\
L & \mapsto L^{1 / 2}
\end{aligned}
$$

é continuamente diferenciável em uma vizinhança aberta da identidade.

Demonstração. Observe que a aplicação $\mathcal{C}$ definida no lema anterior é a inversa da aplicação $\mathcal{R}$. De fato, para $L \in G L_{S}^{+}(H)$, temos

$$
\mathcal{R C}(L)=\mathcal{R}\left(L^{2}\right)=L=\mathcal{C}\left(L^{1 / 2}\right)=\mathcal{C} \mathcal{R}(L)
$$


pois $L$ é definido positivo. Se segue do Lema 5.4.8 que

$$
\mathcal{C}^{\prime}(I) T=2 T \quad \text { para todo } T \in L_{S}(H) .
$$

É claro que $\mathcal{C}^{\prime}(I)$ é um isomorfismo. Como consequência do Teorema da aplicação inversa, existe uma vizinhança aberta $U_{0} \subseteq G L_{S}^{+}(H)$ de $I$ tal que $\mathcal{R}: U_{0} \rightarrow G L_{S}^{+}(H)$ é continuamente diferenciável, como queríamos provar.

Lema 5.4.10. Se $\alpha: J \rightarrow L(H)$ e $\beta: J \rightarrow L(H)$ são duas aplicações continuamente diferenciáveis, então $(\alpha \beta)(\lambda)=\alpha(\lambda) \beta(\lambda): J \rightarrow L(H)$ é continuamente diferenciável $e$, além disso, para $\lambda \in J$ e $v \in \mathbb{R}$,

$$
(\alpha \beta)^{\prime}(\lambda) v=\alpha(\lambda)\left[\beta^{\prime}(\lambda) v\right]+\left[\alpha^{\prime}(\lambda) v\right] \beta(\lambda) .
$$

Podemos ver uma prova do lema anterior, por exemplo, em [18], pág. 336.

Em conclusão do trabalho, apresentaremos um resultado (Teorema 5.4.14) que mostra a conexão entre a forma crossing e o fluxo espectral através de um ponto singular isolado. Antes de enunciar o teorema, precisamos de alguns resultados preliminares.

Observação 5.4.11. Observe que, se $L$ é um operador auto-adjunto com $\|L\|<1$, então $I-L$ é um isomorfismo definido positivo. De fato, se segue do Teorema 1.1.7 que $I-L$ é um isomorfismo. Por outro lado, se $x \in H$ com $\|x\|=1$, temos

$$
\langle x, x\rangle>\|L\|=\sup _{\|z\|=1}|\langle L z, z\rangle| \geq\langle L x, x\rangle,
$$

isto é, $\langle(I-L) x, x\rangle>0$ para todo $x \in H$ com $\|x\|=1$. Consequentemente, $I-L$ é definido positivo.

Teorema 5.4.12. Sejam $P=\left\{P_{\lambda}\right\}_{\lambda \in J}$ um caminho continuamente diferenciável de projeçôes ortogonais e $\lambda_{0}$ um ponto interior de $J$. Então, existem um intervalo $J_{\delta}$ que contém $\lambda_{0}$ e um caminho continuamente diferenciável $U=\left\{U_{\lambda}\right\}_{\lambda \in J_{\delta}}$ de operadores ortogonais, tais que

$$
U_{\lambda_{0}}=I \quad e \quad U_{\lambda} P_{\lambda} U_{\lambda}^{-1}=P_{\lambda_{0}} \quad \text { para todo } \lambda \in J_{\lambda_{0}} .
$$

Demonstração. Fixemos $\lambda_{0}$ no interior de $J$. Para $\lambda \in J$, tomemos

$$
R_{\lambda}=\left(P_{\lambda_{0}}-P_{\lambda}\right)^{2}=P_{\lambda_{0}}+P_{\lambda}-P_{\lambda_{0}} P_{\lambda}-P_{\lambda} P_{\lambda_{0}} .
$$

Então,

$$
\begin{aligned}
R_{\lambda} P_{\lambda_{0}} & =\left(P_{\lambda_{0}}+P_{\lambda}-P_{\lambda_{0}} P_{\lambda}-P_{\lambda} P_{\lambda_{0}}\right) P_{\lambda_{0}}=P_{\lambda_{0}} P_{\lambda_{0}}+P_{\lambda} P_{\lambda_{0}}-P_{\lambda_{0}} P_{\lambda} P_{\lambda_{0}}-P_{\lambda} P_{\lambda_{0}} P_{\lambda_{0}} \\
& =P_{\lambda_{0}}-P_{\lambda_{0}} P_{\lambda} P_{\lambda_{0}}=P_{\lambda_{0}}+P_{\lambda_{0}} P_{\lambda}-P_{\lambda_{0}} P_{\lambda}-P_{\lambda_{0}} P_{\lambda} P_{\lambda_{0}} \\
& =P_{\lambda_{0}}\left(P_{\lambda_{0}}+P_{\lambda}-P_{\lambda_{0}} P_{\lambda}-P_{\lambda} P_{\lambda_{0}}\right)=P_{\lambda_{0}} R_{\lambda} .
\end{aligned}
$$


Analogamente,

$$
R_{\lambda} P_{\lambda}=P_{\lambda} R_{\lambda}
$$

Como o caminho $P$ é contínuo, existe $\delta>0$ tal que

$$
\left\|R_{\lambda}\right\|=\left\|\left(P_{\lambda_{0}}-P_{\lambda}\right)^{2}\right\|<1 \quad \text { para }\left|\lambda-\lambda_{0}\right|<\delta .
$$

Denotemos por $J_{\delta}$ o intervalo $\left(\lambda_{0}-\delta, \lambda_{0}+\delta\right)$. Dado que $R_{\lambda}$ é auto-adjunto e $\left\|R_{\lambda}\right\|<1$ para $\lambda \in J_{\delta}$, da Observação 5.4.11 se segue que $\left\{I-R_{\lambda}\right\}_{J_{\delta}}$ é um caminho de isomorfismos definidos positivos. Assim, existe a raiz quadrada do operador $I-R_{\lambda}$ para $\lambda \in J_{\delta}$. Pela Proposição 5.4.9 podemos supor (diminuindo o intervalo $J_{\delta}$ se for necessário) que $\left\{\left(I-R_{\lambda}\right)^{1 / 2}\right\}_{\lambda \in J_{\delta}}$ é continuamente diferenciável. Tomemos

$$
U_{\lambda}=\left(P_{\lambda} P_{\lambda_{0}}+\left(I-P_{\lambda}\right)\left(I-P_{\lambda_{0}}\right)\right)\left(I-R_{\lambda}\right)^{-1 / 2} \quad \text { para } \lambda \in J_{\delta}
$$

Já que o caminho $P$ é continuamente diferenciável, o Lema 5.4.10, o Teorema 5.4.5 e a Proposição 5.4 .9 implicam que o caminho $U: J_{\delta} \rightarrow L(H)$, definido por $U(\lambda)=U_{\lambda}$, é continuamente diferenciável. Além disso,

$U_{\lambda_{0}}=\left(P_{\lambda_{0}} P_{\lambda_{0}}+\left(I-P_{\lambda_{0}}\right)\left(I-P_{\lambda_{0}}\right)\right)\left(I-\left(P_{\lambda_{0}}-P_{\lambda_{0}}\right)^{2}\right)^{-1 / 2}=\left(P_{\lambda_{0}}+I-P_{\lambda_{0}}\right)^{-1 / 2}=I$.

Provemos que $U_{\lambda}$ é ortogonal para $\lambda \in J_{\delta}$. De fato, dado que $P_{\lambda}$ e $P_{\lambda_{0}}$ são projeções ortogonais, segue-se

$$
\begin{aligned}
U_{\lambda}^{*} & =\left[\left(P_{\lambda} P_{\lambda_{0}}+\left(I-P_{\lambda}\right)\left(I-P_{\lambda_{0}}\right)\right)\left(I-\left(P_{\lambda_{0}}-P_{\lambda}\right)^{2}\right)^{-1 / 2}\right]^{*} \\
& =\left[\left(I-\left(P_{\lambda_{0}}-P_{\lambda}\right)^{2}\right)^{-1 / 2}\right]^{*}\left[\left(P_{\lambda} P_{\lambda_{0}}+\left(I-P_{\lambda}\right)\left(I-P_{\lambda_{0}}\right)\right)\right]^{*} \\
& =\left[\left(I-\left(P_{\lambda_{0}}-P_{\lambda}\right)^{2}\right)^{*}\right]^{-1 / 2}\left[\left(P_{\lambda} P_{\lambda_{0}}\right)^{*}+\left(\left(I-P_{\lambda}\right)\left(I-P_{\lambda_{0}}\right)\right)^{*}\right] \\
& =\left(I-\left(P_{\lambda_{0}}-P_{\lambda}\right)^{2}\right)^{-1 / 2}\left(P_{\lambda_{0}} P_{\lambda}+\left(I-P_{\lambda_{0}}\right)\left(I-P_{\lambda}\right)\right) \\
& =\left(I-R_{\lambda}\right)^{-1 / 2}\left(P_{\lambda_{0}} P_{\lambda}+\left(I-P_{\lambda_{0}}\right)\left(I-P_{\lambda}\right)\right) .
\end{aligned}
$$

Tomemos

$$
U_{\lambda}^{\prime}=P_{\lambda} P_{\lambda_{0}}+\left(I-P_{\lambda}\right)\left(I-P_{\lambda_{0}}\right) \quad \text { e } \quad U_{\lambda}^{\prime \prime}=P_{\lambda_{0}} P_{\lambda}+\left(I-P_{\lambda_{0}}\right)\left(I-P_{\lambda}\right) .
$$

Assim,

$$
\begin{aligned}
U_{\lambda}^{\prime \prime} U_{\lambda}^{\prime} & =\left(P_{\lambda_{0}} P_{\lambda}+\left(I-P_{\lambda_{0}}\right)\left(I-P_{\lambda}\right)\right)\left(P_{\lambda} P_{\lambda_{0}}+\left(I-P_{\lambda}\right)\left(I-P_{\lambda_{0}}\right)\right) \\
& =P_{\lambda_{0}} P_{\lambda}\left(P_{\lambda_{0}}+\left(I-P_{\lambda}\right)\left(I-P_{\lambda_{0}}\right)\right)+\left(I-P_{\lambda_{0}}\right)\left(I-P_{\lambda}\right)\left(P_{\lambda} P_{\lambda_{0}}+\left(I-P_{\lambda_{0}}\right)\right) \\
& =P_{\lambda_{0}} P_{\lambda} P_{\lambda_{0}}+\left(I-P_{\lambda_{0}}\right)\left(I-P_{\lambda}\right)\left(I-P_{\lambda_{0}}\right) \\
& =P_{\lambda_{0}} P_{\lambda} P_{\lambda_{0}}+\left(I-P_{\lambda}-P_{\lambda_{0}}+P_{\lambda_{0}} P_{\lambda}+P_{\lambda} P_{\lambda_{0}}-P_{\lambda_{0}} P_{\lambda} P_{\lambda_{0}}\right) \\
& =I-P_{\lambda_{0}}-P_{\lambda}+P_{\lambda_{0}} P_{\lambda}+P_{\lambda} P_{\lambda_{0}} \\
& =I-\left(P_{\lambda_{0}}-P_{\lambda}\right)^{2} \\
& =I-R_{\lambda} .
\end{aligned}
$$


Analogamente,

$$
U_{\lambda}^{\prime} U_{\lambda}^{\prime \prime}=I-R_{\lambda}
$$

Portanto,

$$
U_{\lambda}^{*} U_{\lambda}=\left(I-R_{\lambda}\right)^{-1 / 2} U_{\lambda}^{\prime \prime} U_{\lambda}^{\prime}\left(I-R_{\lambda}\right)^{-1 / 2}=\left(I-R_{\lambda}\right)^{-1 / 2}\left(I-R_{\lambda}\right)\left(I-R_{\lambda}\right)^{-1 / 2}=I .
$$

Observe que $P_{\lambda}$ e $P_{\lambda_{0}}$ comutam com $\left(I-R_{\lambda}\right)^{-1}$. De fato, como $P_{\lambda}$ e $P_{\lambda_{0}}$ comutam $\operatorname{com} R_{\lambda}$, então

$$
\begin{aligned}
\left(I-R_{\lambda}\right) P_{\lambda} & =P_{\lambda}\left(I-R_{\lambda}\right) \\
\left(I-R_{\lambda}\right)^{-1}\left(I-R_{\lambda}\right) P_{\lambda}\left(I-R_{\lambda}\right)^{-1} & =\left(I-R_{\lambda}\right)^{-1} P_{\lambda}\left(I-R_{\lambda}\right)\left(I-R_{\lambda}\right)^{-1} \\
P_{\lambda}\left(I-R_{\lambda}\right)^{-1} & =\left(I-R_{\lambda}\right)^{-1} P_{\lambda}
\end{aligned}
$$

e

$$
\begin{aligned}
\left(I-R_{\lambda}\right) P_{\lambda_{0}} & =P_{\lambda_{0}}\left(I-R_{\lambda}\right) \\
P_{\lambda_{0}}\left(I-R_{\lambda}\right)^{-1} & =\left(I-R_{\lambda}\right)^{-1} P_{\lambda_{0}} .
\end{aligned}
$$

Daí,

$$
\begin{aligned}
U_{\lambda} U_{\lambda}^{*} & =U_{\lambda}^{\prime}\left(I-R_{\lambda}\right)^{-1 / 2}\left(I-R_{\lambda}\right)^{-1 / 2} U_{\lambda}^{\prime \prime} \\
& =\left[P_{\lambda} P_{\lambda_{0}}+\left(I-P_{\lambda}\right)\left(I-P_{\lambda_{0}}\right)\right]\left(I-R_{\lambda}\right)^{-1}\left[P_{\lambda_{0}} P_{\lambda}+\left(I-P_{\lambda_{0}}\right)\left(I-P_{\lambda}\right)\right] \\
& =\left(I-R_{\lambda}\right)^{-1}\left[P_{\lambda} P_{\lambda_{0}}+\left(I-P_{\lambda}\right)\left(I-P_{\lambda_{0}}\right)\right]\left[P_{\lambda_{0}} P_{\lambda}+\left(I-P_{\lambda_{0}}\right)\left(I-P_{\lambda}\right)\right] \\
& =\left(I-R_{\lambda}\right)^{-1}\left(I-R_{\lambda}\right) \\
& =I .
\end{aligned}
$$

Consequentemente, $U$ é um caminho de operadores ortogonais.

Por último, vejamos que $U_{\lambda} P_{\lambda} U_{\lambda}^{*}=P_{\lambda_{0}}$ para todo $\lambda \in J_{\delta}$. Dado que $P_{\lambda}$ comuta com $\left(I-R_{\lambda}\right)^{-1}$, o Teorema 3.3.14 implica que $P_{\lambda}$ comuta com $\left(I-R_{\lambda}\right)^{-1 / 2}$. Além disso,

$$
U_{\lambda}^{\prime \prime} P_{\lambda}=\left(P_{\lambda_{0}} P_{\lambda}+\left(I-P_{\lambda_{0}}\right)\left(I-P_{\lambda}\right)\right) P_{\lambda}=P_{\lambda_{0}}\left(P_{\lambda_{0}} P_{\lambda}+\left(I-P_{\lambda_{0}}\right)\left(I-P_{\lambda}\right)\right)=P_{\lambda_{0}} U_{\lambda}^{\prime \prime} .
$$

Portanto, como $P_{\lambda_{0}}$ comuta com $\left(I-R_{\lambda}\right)^{-1 / 2}$, então

$$
\begin{aligned}
U_{\lambda}^{*} P_{\lambda} U_{\lambda} & =\left(I-R_{\lambda}\right)^{-1 / 2} U_{\lambda}^{\prime \prime} P_{\lambda} U_{\lambda}^{\prime}\left(I-R_{\lambda}\right)^{-1 / 2} \\
& =\left(I-R_{\lambda}\right)^{-1 / 2} P_{\lambda_{0}} U_{\lambda}^{\prime \prime} U_{\lambda}^{\prime}\left(I-R_{\lambda}\right)^{-1 / 2} \\
& =P_{\lambda_{0}}\left(I-R_{\lambda}\right)^{-1 / 2} U_{\lambda}^{\prime \prime} U_{\lambda}^{\prime}\left(I-R_{\lambda}\right)^{-1 / 2} \\
& =P_{\lambda_{0}}\left(I-R_{\lambda}\right)^{-1 / 2}\left(I-R_{\lambda}\right)\left(I-R_{\lambda}\right)^{-1 / 2} \\
& =P_{\lambda_{0}}
\end{aligned}
$$

o que prova o teorema. 
Teorema 5.4.13. Seja $L=\left\{L_{\lambda}\right\}_{\lambda \in J}$ um caminho de operadores em $\Phi_{S}(H)$. Suponhamos que exista um intervalo fechado $[c, d]$ tal que nem $c$ nem d pertençam ao espectro de $L_{\lambda}$ para $\lambda \in J$. Então, para $\lambda \in J$, existe a projeção $P_{\lambda}$ sobre o subespaço espectral de $L_{\lambda}$ correspondente a $\sigma\left(L_{\lambda}\right) \cap[c, d]$. Além disso, se $L$ é continuamente diferenciável, $P=\left\{P_{\lambda}\right\}_{\lambda \in J}$ é continuamente diferenciável.

Demonstração. Tomemos $\Gamma:[0,1] \rightarrow \mathbb{C}$, definido por

$$
\Gamma(t)=\left(\frac{c+d}{2}, 0\right)+\left(\frac{d-c}{2}\right) e^{i 2 \pi t}
$$

(a circunferência com centro em $\left(\frac{c+d}{2}, 0\right)$ e raio $\left.\frac{d-c}{2}\right)$. Assim, de (4.3.11) se segue

$$
\mathrm{P}_{\lambda}=-\frac{1}{2 \pi i} \int_{\Gamma}\left(\mathrm{L}_{\lambda}-\zeta I\right)^{-1} d \zeta \quad \text { para } \lambda \in J .
$$

No Teorema 4.3.22 mostramos que $\lambda \mapsto \mathrm{P}_{\lambda}$ é contínua.

Suponhamos agora que $\mathrm{L}$ seja continuamente diferenciável e provemos que, para $\lambda_{0} \in J, \dot{\mathrm{P}}_{\lambda_{0}}$ é o operador

$$
\mathrm{B}_{\lambda_{0}}=\frac{1}{2 \pi i} \int_{\Gamma}\left(\mathrm{L}_{\lambda_{0}}-\zeta I\right)^{-1} \dot{\mathrm{L}}_{\lambda_{0}}\left(\mathrm{~L}_{\lambda_{0}}-\zeta I\right)^{-1} d \zeta
$$

(a existência desta integral se deve a que a aplicação definida por

$$
\zeta \mapsto\left(\mathrm{L}_{\lambda_{0}}-\zeta I\right)^{-1} \mathrm{~L}_{\lambda_{0}}\left(\mathrm{~L}_{\lambda_{0}}-\zeta I\right)^{-1}, \quad \text { para } \zeta \in \Gamma,
$$

é contínua). Seja $\varepsilon>0$ dado. Tomemos $\delta>0$ tal que, se $\left|\lambda-\lambda_{0}\right|<\delta$, então

$$
\left\|\frac{\mathrm{L}_{\lambda}-\mathrm{L}_{\lambda_{0}}}{\lambda-\lambda_{0}}-\mathrm{L}_{\lambda_{0}}\right\|<\varepsilon \quad \text { e } \quad\left(\mathrm{L}_{\lambda_{0}}-\zeta I\right)^{-1}-\left(\mathrm{L}_{\lambda}-\zeta I\right)^{-1}<\varepsilon \operatorname{para} \zeta \in \Gamma .
$$

Consideremos

$$
A_{\lambda}=\left(\mathrm{L}_{\lambda}-\zeta I\right)^{-1}\left(\mathrm{~L}_{\lambda}-\mathrm{L}_{\lambda_{0}}\right)\left(\mathrm{L}_{\lambda_{0}}-\zeta I\right)^{-1}-\left(\lambda-\lambda_{0}\right)\left(\mathrm{L}_{\lambda_{0}}-\zeta I\right)^{-1} \dot{\mathrm{L}}_{\lambda_{0}}\left(\mathrm{~L}_{\lambda_{0}}-\zeta I\right)^{-1} .
$$

Assim,

$$
\begin{aligned}
\left\|\frac{A_{\lambda}}{\lambda-\lambda_{0}}\right\|= & \left\|\frac{\left(\mathrm{L}_{\lambda}-\zeta I\right)^{-1}\left(\mathrm{~L}_{\lambda}-\mathrm{L}_{\lambda_{0}}\right)\left(\mathrm{L}_{\lambda_{0}}-\zeta I\right)^{-1}}{\lambda-\lambda_{0}}-\left(\mathrm{L}_{\lambda_{0}}-\zeta I\right)^{-1} \dot{\mathrm{L}}_{\lambda_{0}}\left(\mathrm{~L}_{\lambda_{0}}-\zeta I\right)^{-1}\right\| \\
= & \left\|\left[\frac{\left(\mathrm{L}_{\lambda}-\zeta I\right)^{-1}\left(\mathrm{~L}_{\lambda}-\mathrm{L}_{\lambda_{0}}\right)}{\lambda-\lambda_{0}}-\left(\mathrm{L}_{\lambda_{0}}-\zeta I\right)^{-1} \dot{\mathrm{L}}_{\lambda_{0}}\right]\left(\mathrm{L}_{\lambda_{0}}-\zeta I\right)^{-1}\right\| \\
\leq & \left\|\left(\mathrm{L}_{\lambda}-\zeta I\right)^{-1}\left[\frac{\mathrm{L}_{\lambda}-\mathrm{L}_{\lambda_{0}}}{\lambda-\lambda_{0}}-\dot{\mathrm{L}}_{\lambda_{0}}\right]+\left(\left(\mathrm{L}_{\lambda_{0}}-\zeta I\right)^{-1}-\left(\mathrm{L}_{\lambda}-\zeta I\right)^{-1}\right) \dot{\mathrm{L}}_{\lambda_{0}}\right\| \\
& \cdot\left\|\left(\mathrm{L}_{\lambda_{0}}-\zeta I\right)^{-1}\right\| \\
\leq & {\left[\left\|\left(\mathrm{L}_{\lambda}-\zeta I\right)^{-1}\right\|+\left\|\dot{\mathrm{L}}_{\lambda_{0}}\right\|\right]\left\|\left(\mathrm{L}_{\lambda_{0}}-\zeta I\right)^{-1}\right\| \varepsilon } \\
\leq & {\left[M+\left\|\dot{\mathrm{L}}_{\lambda_{0}}\right\|\right] M \varepsilon, }
\end{aligned}
$$


onde

$$
M=\sup _{\lambda \in J, \zeta \in \Gamma}\left\|\left(\mathrm{L}_{\lambda}-\zeta I\right)^{-1}\right\| .
$$

Por outro lado, para $\lambda \in J$, temos

$$
\begin{aligned}
\mathrm{P}_{\lambda}-\mathrm{P}_{\lambda_{0}} & =-\frac{1}{2 \pi i} \int_{\Gamma}\left(\mathrm{L}_{\lambda}-\zeta I\right)^{-1} d \zeta+\frac{1}{2 \pi i} \int_{\Gamma}\left(\mathrm{L}_{\lambda_{0}}-\zeta I\right)^{-1} d \zeta \\
& =-\frac{1}{2 \pi i} \int_{\Gamma}\left(\left(\mathrm{L}_{\lambda}-\zeta I\right)^{-1}-\left(\mathrm{L}_{\lambda_{0}}-\zeta I\right)^{-1}\right) d \zeta \\
& =\frac{1}{2 \pi i} \int_{\Gamma}\left(\mathrm{L}_{\lambda}-\zeta I\right)^{-1}\left(\mathrm{~L}_{\lambda}-\zeta I-\mathrm{L}_{\lambda_{0}}+\zeta I\right)\left(\mathrm{L}_{\lambda_{0}}-\zeta I\right)^{-1} d \zeta \\
& =\frac{1}{2 \pi i} \int_{\Gamma}\left(\mathrm{L}_{\lambda}-\zeta I\right)^{-1}\left(\mathrm{~L}_{\lambda}-\mathrm{L}_{\lambda_{0}}\right)\left(\mathrm{L}_{\lambda_{0}}-\zeta I\right)^{-1} d \zeta
\end{aligned}
$$

Consequentemente, pelo Lema 4.3.21,

$$
\left\|\frac{\mathrm{P}_{\lambda}-\mathrm{P}_{\lambda_{0}}}{\lambda-\lambda_{0}}-\mathrm{B}_{\lambda_{0}}\right\|=\left\|\frac{1}{2 \pi i} \int_{\Gamma} \frac{A_{\lambda}}{\lambda-\lambda_{0}} d \zeta\right\| \leq \frac{1}{2 \pi}\left[M+\left\|\dot{\mathrm{L}}_{\lambda_{0}}\right\|\right] M l \varepsilon,
$$

onde $l$ é o comprimento de $\Gamma$. Em conclusão, $\mathrm{B}_{\lambda_{0}}=\dot{\mathrm{P}}_{\lambda_{0}}$.

Por último, vejamos a continuidade de $\dot{\mathrm{P}}$. Fixemos $\lambda_{0}$ em $J$. Seja $\varepsilon>0$ dado. Pela continuidade de $\lambda \mapsto\left(\mathrm{L}_{\lambda}-\zeta I\right)^{-1} \dot{\mathrm{L}}_{\lambda}\left(\mathrm{L}_{\lambda}-\zeta I\right)^{-1}$, para $\lambda \in J\left(\lambda \mapsto \dot{\mathrm{L}}_{\lambda}\right.$ é contínua por hipótese), existe $\delta>0$ tal que, se $\left|\lambda-\lambda_{0}\right|<\delta$,

$$
\left\|\left(\mathrm{L}_{\lambda_{0}}-\zeta I\right)^{-1} \dot{\mathrm{L}}_{\lambda_{0}}\left(\mathrm{~L}_{\lambda_{0}}-\zeta I\right)^{-1}-\left(\mathrm{L}_{\lambda}-\zeta I\right)^{-1} \dot{\mathrm{L}}_{\lambda}\left(\mathrm{L}_{\lambda}-\zeta I\right)^{-1}\right\|<\varepsilon .
$$

Portanto,

$$
\begin{aligned}
\left\|\dot{\mathrm{P}}_{\lambda_{0}}-\dot{\mathrm{P}}_{\lambda}\right\| & =\left\|\frac{1}{2 \pi i} \int_{\Gamma}\left[\left(\mathrm{L}_{\lambda_{0}}-\zeta I\right)^{-1} \dot{\mathrm{L}}_{\lambda_{0}}\left(\mathrm{~L}_{\lambda_{0}}-\zeta I\right)^{-1}-\left(\mathrm{L}_{\lambda}-\zeta I\right)^{-1} \dot{\mathrm{L}}_{\lambda}\left(\mathrm{L}_{\lambda}-\zeta I\right)^{-1}\right] d \zeta\right\| \\
& \leq \frac{1}{2 \pi} l \varepsilon
\end{aligned}
$$

e a prova é concluída.

Finalmente, como se tinha falado acima, provaremos que, se $\lambda_{0}$ é um ponto singular regular do $\mathrm{L}$, isto é, $\lambda_{0}$ é isolado em $\Sigma(\mathrm{L})$ e a forma crossing $Q\left(\mathrm{~L}, \lambda_{0}\right)$ é não degenerada, então o fluxo espectral de $\mathrm{L}$ em $\lambda_{0}$ coincide com a assinatura da forma crossing de $\mathrm{L}$ em $\lambda_{0}$.

Teorema 5.4.14. Sejam $\lambda_{0}$ no interior de $J$ um ponto singular regular de $L$ e $Q\left(L, \lambda_{0}\right)$ a forma crossing de $L$ em $\lambda_{0}$. Então,

$$
\operatorname{sf}\left(L, \lambda_{0}\right)=\operatorname{sign} Q\left(L, \lambda_{0}\right)=\operatorname{sign}\left(\left.\dot{L}_{\lambda_{0}}\right|_{\operatorname{Ker} L_{\lambda_{0}}}\right) .
$$


Demonstração. Como provamos no começo da seção, dado que, por hipótese, $\lambda_{0}$ é isolado em $\Sigma(\mathrm{L})$, então $\operatorname{sf}\left(\mathrm{L}, \lambda_{0}\right)$ existe. Além disso, 0 é isolado em $\sigma\left(\mathrm{L}_{\lambda_{0}}\right)$ pela Proposição 3.4.3. Portanto, podemos escolher $\varepsilon>0$ suficientemente pequeno tal que o único ponto no espectro de $L_{\lambda_{0}}$ em $[-\varepsilon, \varepsilon]$ seja 0 (0 pertence a $\sigma\left(L_{\lambda_{0}}\right)$ pois $L_{\lambda_{0}}$ não é inversível). Daí,

$$
\mathrm{L}_{\lambda_{0}}-\alpha I \text { é inversível para todo } \alpha \in[-\varepsilon, \varepsilon]-\{0\} .
$$

Assim, pela continuidade de $L$, existe $\rho>0$ tal que $L_{\lambda}-\varepsilon I$ e $L_{\lambda}+\varepsilon I$ são inversíveis para $\lambda \in J_{\rho}=\left[\lambda_{0}-\rho, \lambda_{0}+\rho\right]$. Logo, se $\lambda \in J_{\rho}$, se segue que $\varepsilon$ e $-\varepsilon$ não pertencem ao espectro de $L_{\lambda}$. Consequentemente, para $\lambda \in J_{\rho}$, podemos definir a projeção ortogonal sobre o subespaço espectral de $\mathrm{L}_{\lambda}$ correspondente a $\sigma\left(\mathrm{L}_{\lambda}\right) \cap[-\varepsilon, \varepsilon]$ (ver Teorema 5.4.13). Denotemos por $P_{\lambda}$ esta projeção. Como $L$ é continuamente diferenciável, o Teorema 4.3.22 implica que $\mathrm{P}=\left\{\mathrm{P}_{\lambda}\right\}_{\lambda \in J_{\rho}}$ é um caminho continuamente diferenciável de projeções ortogonais. Do Teorema 5.4.12 temos que existe um intervalo aberto $J_{\lambda_{0}} \subseteq J_{\rho}$ que contém $\lambda_{0}$ e um caminho continuamente diferenciável $\mathrm{U}=\left\{\mathrm{U}_{\lambda}\right\}_{\lambda \in J_{\lambda_{0}}}$ de operadores ortogonais, tais que

$$
\mathrm{U}_{\lambda_{0}}=I \quad \text { e } \quad \mathrm{U}_{\lambda} \mathrm{P}_{\lambda} \mathrm{U}_{\lambda}^{-1}=\mathrm{P}_{\lambda_{0}} \quad \text { para todo } \lambda \in J_{\lambda_{0}} .
$$

Se segue da invariância do fluxo espectral pela ação cogradiente que

$$
\operatorname{sf}\left(\mathrm{L}, \lambda_{0}\right)=\operatorname{sf}\left(\mathrm{ULU}^{-1}, \lambda_{0}\right) .
$$

Denotemos por $H_{0}$ o espaço Ker $L_{\lambda_{0}}$. De (4.3.13) obtemos $L_{\lambda} P_{\lambda}=P_{\lambda} L_{\lambda}$. Logo, para cada $\lambda \in J_{\lambda_{0}}, \operatorname{Ker}\left(\mathrm{P}_{\lambda}\right)$ e $\operatorname{Ker}\left(\mathrm{P}_{\lambda}\right)^{\perp}$ são invariantes por $\mathrm{L}_{\lambda}$. Portanto, a relação (5.4.6) implica que

$$
U_{\lambda} L_{\lambda} U_{\lambda}^{-1} P_{\lambda_{0}}=U_{\lambda} L_{\lambda} U_{\lambda}^{-1} U_{\lambda} P_{\lambda} U_{\lambda}^{-1}=U_{\lambda} L_{\lambda} P_{\lambda} U_{\lambda}^{-1}=U_{\lambda} P_{\lambda} L_{\lambda} U_{\lambda}^{-1}=P_{\lambda_{0}} U_{\lambda} L_{\lambda} U_{\lambda}^{-1} .
$$

Daí, $H_{0}$ e $H_{0}^{\perp}$ são invariantes por $\mathrm{U}_{\lambda} \mathrm{L}_{\lambda} \mathrm{U}_{\lambda}^{-1}$. Como, por hipótese, $\mathrm{L}_{\lambda}$ é um isomorfismo para $\lambda$ suficientemente perto de $\lambda_{0} \operatorname{com} \lambda \neq \lambda_{0}$, então o fato anterior implica que a restrição

$$
\mathrm{U}_{\lambda} \mathrm{L}_{\lambda} \mathrm{U}_{\lambda}^{-1}: H_{0}^{\perp} \rightarrow H_{0}^{\perp}
$$

é um isomorfismo para todo $\lambda$ suficientemente perto de $\lambda_{0}$. Assim, das propriedades do fluxo espectral do produto direto e da normalização do fluxo espectral, obtemos que

$$
\operatorname{sf}\left(\mathrm{ULU}^{-1}, \lambda_{0}\right)=\operatorname{sf}\left(\left.\mathrm{ULU}^{-1}\right|_{H_{0}}, \lambda_{0}\right)+\operatorname{sf}\left(\left.\mathrm{ULU}^{-1}\right|_{H_{0}^{\perp}}, \lambda_{0}\right)=\operatorname{sf}\left(\left.\mathrm{ULU}^{-1}\right|_{H_{0}}, \lambda_{0}\right) .
$$

Tomemos $\ell_{\lambda}=\left.U_{\lambda} L_{\lambda} U_{\lambda}^{-1}\right|_{H_{0}}$. Como sf $\left(\mathrm{L}, \lambda_{0}\right)=\operatorname{sf}\left(\mathrm{ULU}^{-1}, \lambda_{0}\right)$, a fórmula anterior implica que

$$
\operatorname{sf}\left(\mathrm{L}, \lambda_{0}\right)=\operatorname{sf}\left(\ell, \lambda_{0}\right) .
$$

Se segue do Lema 5.4.10 que, para $\lambda \in J$,

$$
\dot{\ell}_{\lambda}=\left.\dot{U}_{\lambda} \mathrm{L}_{\lambda} \mathrm{U}_{\lambda}^{-1}\right|_{H_{0}}+\left.\mathrm{U}_{\lambda} \dot{\mathrm{L}}_{\lambda} \mathrm{U}_{\lambda}^{-1}\right|_{H_{0}}+\left.\mathrm{U}_{\lambda} \mathrm{L}_{\lambda} \dot{\mathrm{U}}_{\lambda}^{-1}\right|_{H_{0}} .
$$


Então, já que $\mathrm{L}_{\lambda_{0}}$ é auto-adjunto e $\mathrm{U}_{\lambda_{0}}=I$, para $u$ e $v$ em $\operatorname{Ker} \mathrm{L}_{\lambda_{0}}=\operatorname{Ker} \ell_{\lambda_{0}}$ temos

$$
\begin{aligned}
\left\langle\dot{\ell}_{\lambda_{0}} u, v\right\rangle & =\left\langle\left[\left.\dot{\mathrm{U}}_{\lambda_{0}} \mathrm{~L}_{\lambda_{0}} \mathrm{U}_{\lambda_{0}}^{-1}\right|_{H_{0}}+\left.\mathrm{U}_{\lambda_{0}} \dot{\mathrm{L}}_{\lambda_{0}} \mathrm{U}_{\lambda_{0}}^{-1}\right|_{H_{0}}+\left.\mathrm{U}_{\lambda_{0}} \mathrm{~L}_{\lambda_{0}} \dot{\mathrm{U}}_{\lambda_{0}}^{-1}\right|_{H_{0}}\right] u, v\right\rangle \\
& =\left\langle\left[\left.\dot{\mathrm{U}}_{\lambda_{0}} \mathrm{~L}_{\lambda_{0}}\right|_{H_{0}}+\left.\dot{\mathrm{L}}_{\lambda_{0}}\right|_{H_{0}}+\left.\mathrm{L}_{\lambda_{0}} \dot{\mathrm{U}}_{\lambda_{0}}^{-1}\right|_{H_{0}}\right] u, v\right\rangle \\
& =\left\langle\left.\dot{\mathrm{U}}_{\lambda_{0}} \mathrm{~L}_{\lambda_{0}}\right|_{H_{0}} u, v\right\rangle+\left\langle\left.\dot{\mathrm{L}}_{\lambda_{0}}\right|_{H_{0}} u, v\right\rangle+\left\langle\left.\mathrm{L}_{\lambda_{0}} \dot{\mathrm{U}}_{\lambda_{0}}^{-1}\right|_{H_{0}} u, v\right\rangle \\
& =\left\langle\left.\dot{\mathrm{L}}_{\lambda_{0}}\right|_{H_{0}} u, v\right\rangle+\left\langle\left.\dot{\mathrm{U}}_{\lambda_{0}}^{-1}\right|_{H_{0}} u, \mathrm{~L}_{\lambda_{0}} v\right\rangle \\
& =\left\langle\left.\dot{\mathrm{L}}_{\lambda_{0}}\right|_{H_{0}} u, v\right\rangle \\
& =\left\langle\dot{\mathrm{L}}_{\lambda_{0}} u, v\right\rangle .
\end{aligned}
$$

Consequentemente, as formas crossing de $\mathrm{L}$ e $\ell$ em $\lambda_{0}$ são iguais, isto é,

$$
Q\left(\mathrm{~L}, \lambda_{0}\right)=Q\left(\ell, \lambda_{0}\right)
$$

De (5.4.7) e (5.4.8) temos que a prova estará completa se mostrarmos que

$$
\operatorname{sf}\left(\ell, \lambda_{0}\right)=\operatorname{sign} Q\left(\ell, \lambda_{0}\right)
$$

Como, por hipótese, a forma $Q\left(\mathrm{~L}, \lambda_{0}\right)$ é não degenerada, da igualdade (5.4.8) temos que a forma $Q\left(\ell, \lambda_{0}\right)$ em $H_{0}$ é também não degenerada. Daí, $\dot{\ell}_{\lambda_{0}}$ é um operador inversível em $H_{0}$. Assim, já que $G L\left(H_{0}\right)$ é aberto, existe $\alpha>0$ tal que

$$
\dot{\ell}_{\lambda_{0}}+A \text { é inversível para } A \in L\left(H_{0}\right) \text { com }\|A\|<\alpha \text {. }
$$

Por outro lado, podemos escolher $\delta>0$ tal que

$$
\left\|\frac{\ell_{\lambda}}{\lambda-\lambda_{0}}-\dot{\ell}_{\lambda_{0}}\right\|=\left\|\frac{\ell_{\lambda}-\ell_{\lambda_{0}}}{\lambda-\lambda_{0}}-\dot{\ell}_{\lambda_{0}}\right\|<\alpha
$$

sempre que $0<\left|\lambda-\lambda_{0}\right|<2 \delta\left(\ell_{\lambda_{0}}=0\right.$ pela definição de $\left.\ell\right)$. Tomemos a homotopia $\mathrm{h}:[0,1] \times\left[\lambda_{0}-\delta, \lambda_{0}+\delta\right] \rightarrow L\left(H_{0}\right)$ definida por

$$
\mathrm{h}(t, \lambda)=(1-t)\left(\lambda-\lambda_{0}\right) \dot{\ell}_{\lambda_{0}}+t \ell_{\lambda}=\left[\lambda-\lambda_{0}\right]\left[\dot{\ell}_{\lambda_{0}}+t\left(\ell_{\lambda} /\left(\lambda-\lambda_{0}\right)-\dot{\ell}_{\lambda_{0}}\right)\right]
$$

Dado que

$$
\left\|t\left[\left(\ell_{\lambda}-\ell_{\lambda_{0}}\right) /\left(\lambda-\lambda_{0}\right)-\dot{\ell}_{\lambda_{0}}\right]\right\|<\alpha \quad \text { para } 0<\left|\lambda-\lambda_{0}\right|<2 \delta \text { e } t \in[0,1]
$$

então (5.4.10) implica que $\mathrm{h}(t, \lambda)$ é inversível para $0 \leq t \leq 1$ e $\lambda=\lambda_{0} \pm \delta$. Portanto, para $0 \leq t \leq 1$ o caminho $\mathrm{h}_{t}=\mathrm{h}(t, \cdot)$ é admissível. Da invariância homotópica do fluxo espectral se segue

$$
\operatorname{sf}\left(\ell, \lambda_{0}\right)=\operatorname{sf}\left(h_{1}, \lambda_{0}\right)=\operatorname{sf}\left(h_{0}, \lambda_{0}\right)=\operatorname{sf}\left(\left(\lambda-\lambda_{0}\right) \dot{\ell}_{\lambda_{0}}, \lambda_{0}\right)
$$


Agora, do Corolário 5.3.5 temos

$$
\begin{aligned}
\operatorname{sf}\left(\left(\lambda-\lambda_{0}\right) \dot{\ell}_{\lambda_{0}}, \lambda_{0}\right) & =\mu\left(\left(\lambda_{0}-\delta-\lambda_{0}\right)\left(\dot{\ell}_{\lambda_{0}}\right)\right)-\mu\left(\left(\lambda_{0}+\delta-\lambda_{0}\right)\left(\dot{\ell}_{\lambda_{0}}\right)\right) \\
& =\mu\left(-\delta\left(\dot{\ell}_{\lambda_{0}}\right)\right)-\mu\left(\delta\left(\dot{\ell}_{\lambda_{0}}\right)\right) \\
& =\mu\left(-\dot{\ell}_{\lambda_{0}}\right)-\mu\left(\dot{\ell}_{\lambda_{0}}\right) \\
& =\operatorname{sign} \dot{\ell}_{\lambda_{0}}
\end{aligned}
$$

e assim, $\operatorname{sf}\left(\ell, \lambda_{0}\right)=\operatorname{sign} \dot{\ell}_{\lambda_{0}}$, como queríamos provar. 


\section{Bibliografia}

[1] A. Abbondandolo, "Morse Theory for Hamiltonian Systems", Chapman \& Hall/CRC, Boca Raton, London, New York, Washington, D.C, 2001.

[2] L. V. Ahlfors, "Compelx Analysis: An Introduction to the Theory of Analytic Functions of One Complex Variable", McGraw-Hill, New York, 1979.

[3] T. M. Apostol, "Calculus", Vol. II, Second Sedition, Multi Variable Calculus and Linear Algebra, with Applications to Differential Equations and Probability, Jhon Wiley \& Sons, New York, London, Sydney, Toronto, 1969.

[4] T. M. Apostol, "Mathematical Analysis", Second Edition, 1974.

[5] M. F. Atiyah, V. K. Patodi e I.M. Singer, Spectral Asymmetry and Riemannian Geometry, III, Proc. Cambridge Philos. Soc. 79 (1976), 71-99.

[6] N. C. Bernardes e C. S. Fernandez, "Introdução às Funções de uma Variável Complexa, textos Universitários, Sociedade Brasileira de Matemática, 2008.

[7] J. B. Conway, "A Course in Functional Analysis", Second Edition, Graduate Texs in Mathematics, Springer-Verlag, New York, 1990.

[8] M. Fabian, P. Habala, P. Hájek, V. Montesinos, J. Pelant, V. Zizler, "Functional Analysis and Infinite-Dimensional Geometry", Canadian Mathematical Society, Springer-Verlag, New York, 2001.

[9] P. M. Fitzpatrick, J. Pejsachowicz e L. Recht, Spectral flow and Bifurcation of Critical Points of Strongly-Indefinite Functionals Part I. General Theory, Journal of Functional Analysis 162, 52-95, Academic Press, (1999).

[10] I. Gohberg, S. Goldberg, M. A. Kaashoek, "Basic Classes of Linear Operators", Birkhäuser Verlag, Basel, Boston, Berlin, 2003.

[11] K. Hoffman and R. Kunze, "Algebra Linear", Prentice-Hall Hispanoamericana, S. A., México, Londres, Sydney, Toronto, Nueva Delhi, Tokio, Singapur, Rio de Janeiro, 1973. 
[12] K. Jänich, "Topology", Undergraduate Texts in Mathematics, Springer-Verlag, New York, 1984.

[13] T. Kato, "Perturbation Theory for Linear Operators", Grundlehren der Mathematischen Wissenschaften, Vol. 132, Springer-Verlag, New York/Berlin, 1980.

[14] P. Kirwan, "Complexification of Multilinear and Polinonial Mappings on Normed Spaces", Departament of Mathematics, National University of Ireland, Gawlay, 1997.

[15] K. Knopp, "Theory of Functions", Part one, Elements of the General Theory of Analytic Functions, Dover Publications, New York, 1945.

[16] E. Kreyszig, "Introductory Functional Analysis with Applications", John Wiley \& Sons, New York, Santa Barbara, London, Sydney, Toronto, 1978.

[17] N. H. Kuiper, The Homotopy Type of the Unitary Group of Hilbert Space, Topology Vol. 3 (1965), pp. 19-30. Pergamon Press, Printed in Great Britain.

[18] S. Lang, "Real and Functional Analysis", Third Edition, Graduate Texs in Mathematics, Springer-Verlag, New York, 1993.

[19] E. L. Lima, "Variedades Diferenciáveis", Publicações Matemáticas, IMPA, 2011.

[20] J. Mawhin e M. Willem, "Critical Point Theory and Hamiltonial System", Applied Mathematical Sciences, Vol. 483, No. 483, Springer-Verlag, New York/Berlin, 1989.

[21] J. S. Milne, "Group Theory", Version 3.13, March 15, 2013.

[22] J. R. Munkres, "Topology", Second Edition, Massachusetts Institute of Tecnology, Prentice Hall, Inc, 2000.

[23] H. Osborn, "Vector Bundles", Vol. I, Fundations and Stiefel- Whitney Classes, Pure and Applied Mathematics, Academis Press, New York, London, Paris, San Diego, San Francisco, São Paulo, Sydney, Tokyo, Toronto, 1982.

[24] M. Schechter, "Principles of Functional Analysis", Second Edition, Graduate Studies in Mathematics, Vol. 36. American Mathematics Society, Providence, Rhode Island, 2002.

[25] M. Spivak, "Calculus on Manifolds", A Modern Aproach to Classical theorems of Advanced Calculus, Addison - Wesley Publishing Company, 1965.

[26] A. E. Taylor, "Introduction to Functional", New York-John Wiley \& Sons, Inc. London, Chapman \& Hall, Ltd, 1958. 
[27] F. W. Warner, "Foundations of Differentiable Manifolds and Lie Groups", Springer-Verlag, New York, 1971. 